\title{
KRW Oxygen-Blown Gasification Combined Cycle: Carbon Dioxide Recovery, Transport, and Disposal
}

by R.D. Doctor, J.C. Molburg, ${ }^{*}$ and P.R. Thimmapuram*

Energy Systems Division,

Argonne National Laboratory, 9700 South Cass Avenue, Argonne, Illinois 60439

August 1996

Work sponsored by United States Department of Energy,

Assistant Secretary for Fossil Energy

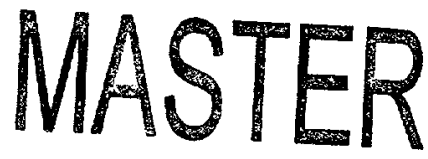

*Molburg and Thimmapuram are affiliated with Argonne's Decision and Information Sciences Division. 
This report is printed on recycled paper. 


\section{DISCLAIMER}

Portions of this document may be illegible in electronic image products. Images are produced from the best available original document. 



\section{Contents}

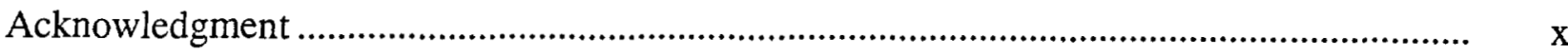

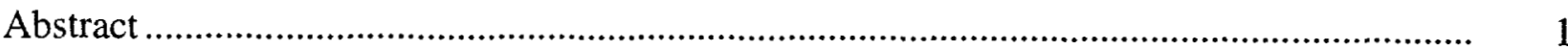

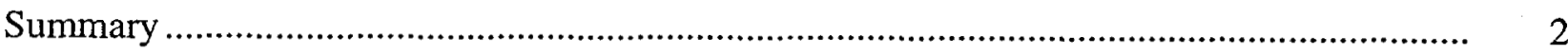

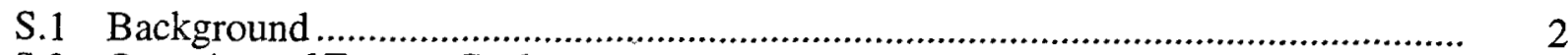

S.2 Overview of Energy Cycle .....................................................................................

S.3 Mining, Preparation, and Transportation of Raw Materials .................................... 3

S.4 Handling of Coal and Limestone ..........................................................................

S.5 Base Cases for Integrated Gasification Combined Cycle ......................................... 5

S.5.1 Gasifier Island ...................................................................................

S.5.2 Power Island........................................................................................... 8

S.6 Integrated Gasification Combined Cycle with $\mathrm{CO}_{2}$ Recovery ……..........................

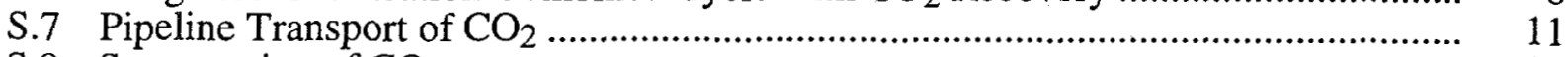

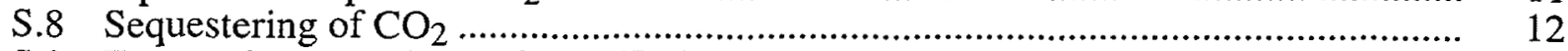

S.9 Energy Consumption and $\mathrm{CO}_{2}$ Emissions ....................................................... 12

S.10 Economic Summary ……............................................................................... 12

S.11 References for Summary .................................................................................... 12

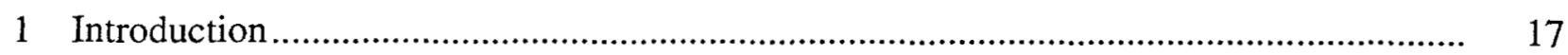

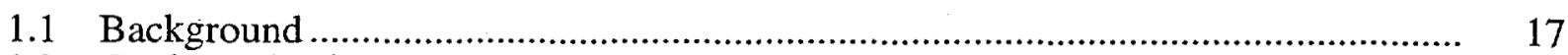

1.2 Goals, Objectives, and Approach...................................................................... 17

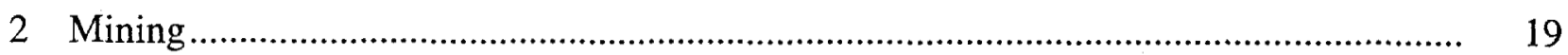

2.1 Mining, Preparation, and Transportation of Raw Materials .................................... 19

2.2 Coal and Limestone Handling................................................................................ 19

3 Oxygen-Blown Base Case with $\mathrm{No}^{\mathrm{CO}_{2}}$ Recovery ....................................................... 21

3.1 Design Basis.............................................................................................. 21

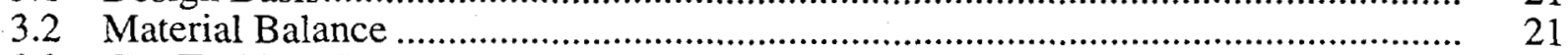

3.3 Gas Turbine, Steam Cycle, and Plant Performance ................................................. 23

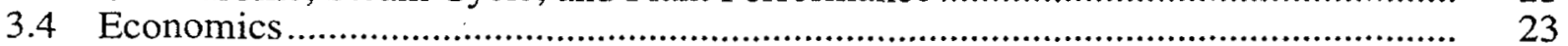

4 Case 1 - Gas Turbine Topping Cycle and Glycol $\mathrm{CO}_{2}$ Recovery ................................. 24

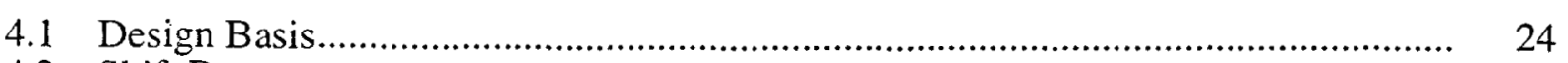

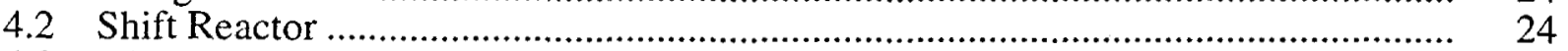

4.3 Glycol Process for $\mathrm{CO}_{2}$ and $\mathrm{H}_{2} \mathrm{~S}$ Recovery ........................................................ 27

4.4 Gas Turbine, Steam Cycle, and Plant Performance ................................................... 27

4.5 Economics ...................................................................................................... 49

5 Case 2 - Gas Turbine Topping Cycle and Membrane $\mathrm{CO}_{2}$ Recovery ............................. 64

5.1 Design Basis................................................................................................... 64

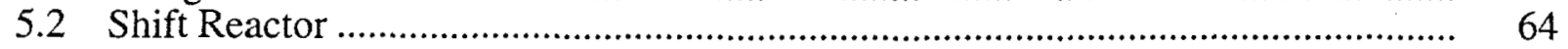




\section{Contents (Cont.)}

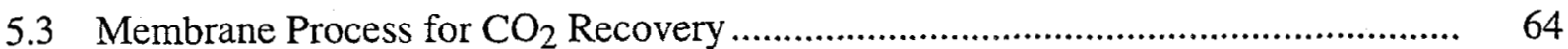

5.4 Gas Turbine, Steam Cycle, and Plant Performance .............................................. 66

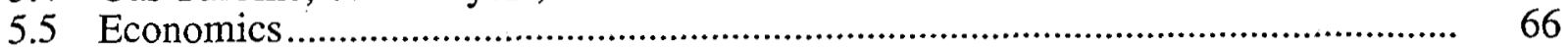

6 Case 3 - Fuel Cell Topping Cycle and Glycol $\mathrm{CO}_{2}$ Recovery ............................... 83

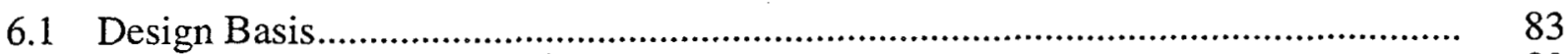

6.2 Chilled Methanol Process for $\mathrm{H}_{2} \mathrm{~S}$ Recovery ............................................ 83

6.3 Molten Carbonate Fuel Cell System ................................................................... 85

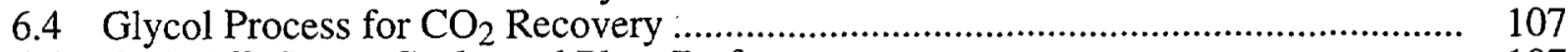

6.5 Fuel Cell, Steam Cycle, and Plant Performance ........................................... 107

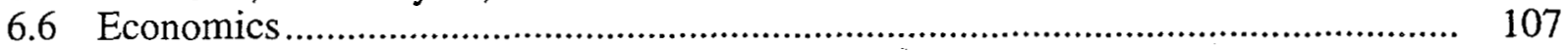

7 Case 4 - Fuel Cell Topping Cycle and Membrane $\mathrm{CO}_{2}$ Recovery ............................ 133

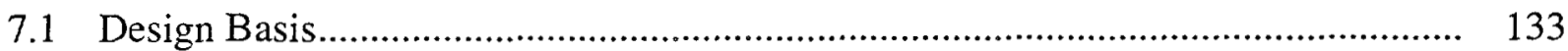

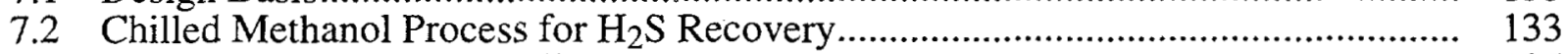

7.3 Molten Carbonate Fuel Cell System ................................................................ 135

7.4 Membrane System for $\mathrm{CO}_{2}$ Recovery .......................................................... 135

7.5 Fuel Cell, Steam Cycle, and Plant Performance ........................................... 135

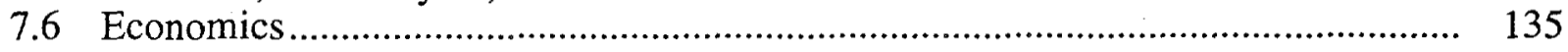

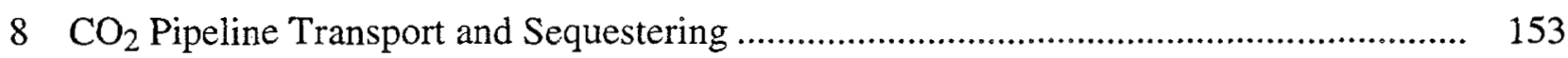

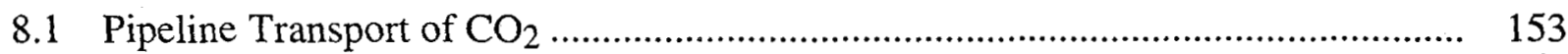

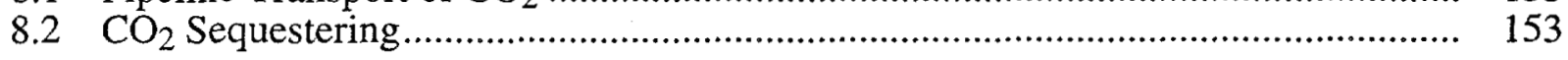

9 Conclusions - Energy Cycle/Economic Comparisons ..................................... 154

9.1 Energy Consumption and $\mathrm{CO}_{2}$ Emissions .................................................. 154

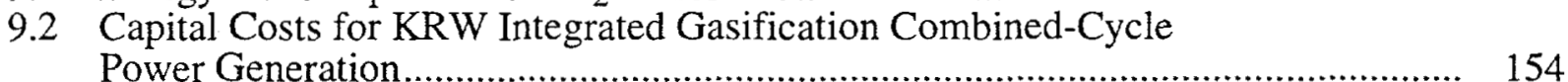

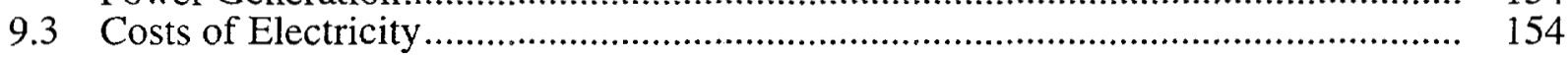

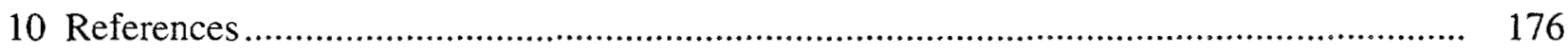

Figures

S.1 Simplified Overview of the Energy Cycle Components for $\mathrm{CO}_{2}$ Recovery ................ 4

S.2 Block Diagram of the Base-Case Oxygen-Blown KRW IGCC System ...................... 6

S.3 Block Diagram of the Base-Case Air-Blown KRW IGCC System........................... 7 


\section{Figures (Cont.)}

S.4 Block Diagram of the Base-Case Oxygen-Blown KRW IGCC System

Modified for $\mathrm{CO}_{2}$ Recovery

S.5 Block Diagram of the Base-Case Air-Blown KRW IGCC System Modified for $\mathrm{CO}_{2}$ Recovery

3.1 Block Diagram of the Base-Case Oxygen-Blown KRW IGCC System

4.1 Block Diagram of the Base-Case Oxygen-Blown KRW IGCC System

Modified for $\mathrm{CO}_{2}$ Recovery

4.2 Gas Stream Composition at Various Stages in the Process in Case 1......................... 26

4.3 Flow Diagram of Shift System and Associated Heat Integration in Case 1................. 28

4.4 Flow Diagram of Glycol Process for $\mathrm{H}_{2} \mathrm{~S}$ Recovery in Case 1 ............................... 33

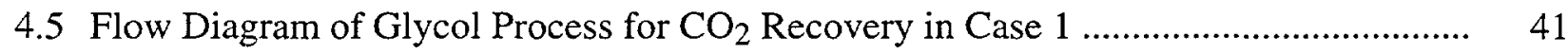

5.1 Flow Diagram of Membrane Process for $\mathrm{CO}_{2}$ Recovery in Case 2 ......................... 65

6.1 Block Diagram of the IGCC System with $\mathrm{CO}_{2}$ Recovery Used in Cases 3 and $4 \ldots \ldots \ldots . . \quad 84$

6.2 Flow Diagram of Chilled Methanol Process for $\mathrm{H}_{2} \mathrm{~S}$ Recovery in Case 3.................... 86

6.3 Flow Diagram of Fuel Cell System and Associated Heat Recovery in Case 3 .............. 95

6.4 Flow Diagram of Glycol Process for $\mathrm{CO}_{2}$ Recovery and Chilled Methanol Process for $\mathrm{H}_{2} \mathrm{~S}$ Recovery with Fuel Cell Topping Cycle in Case 3 .......................... 108

7.1 Block Diagram of the IGCC System with $\mathrm{CO}_{2}$ Recovery Used in Cases 3 and $4 \ldots \ldots \ldots . .134$

7.2 Flow Diagram of Membrane Process for $\mathrm{CO}_{2}$ Removal in Case 4 ............................ 140

\section{Tables}

S.1 Energy Consumption and $\mathrm{CO}_{2}$ Emissions for Oxygen-Blown Base Case with No $\mathrm{CO}_{2}$ Recovery

S.2 Energy Consumption and $\mathrm{CO}_{2}$ Emissions for Optimal Oxygen-Blown Case with $\mathrm{CO}_{2}$ Recovery: Case 1

S.3 Summary of Comparative Costs of IGCC Systems 


\section{Tables (Cont.)}

1.1 Alternative Plant Configurations ............................................................................ 18

2.1 Analysis of Coal from Illinois No. 6 Seam, Old Ben No. 26 Mine................................ 20

3.1 Material Flows for Oxygen-Blown and Air-Blown Base Cases...................................... 23

4.1 Material Flows for Oxygen-Blown Base Case and Case 1 ............................................. 27

4.2 Heat Recovery and Allocation for Gas Turbine/Glycol Process in Case 1..................... 29

4.3 Stream Flows of Shift System of Gas Turbine/Glycol Process in Case 1 ….................. 30

4.4 Stream Flows of Glycol Process for $\mathrm{H}_{2} \mathrm{~S}$ Removal in Case 1 ..................................... 34

4.5 Descriptions of Streams of Glycol Process for $\mathrm{H}_{2} \mathrm{~S}$ Removal in Case 1 ........................ 37

4.6 Stream Flows of Glycol Process for $\mathrm{CO}_{2}$ Removal in Case 1 .................................. 42

4.7 Descriptions of Streams of Glycol Process for $\mathrm{CO}_{2}$ Removal in Case 1 ........................ 45

4.8 Power Output, Plant Power Use, and Net Power Output for Base Case and Case 1 Gas Turbine/Glycol Process ..................................................................................

4.9 Sizing and Cost Estimation for Major Equipment Used for $\mathrm{H}_{2} \mathrm{~S}$ Removal in Glycol Process in Case 1.

4.10 Sizing and Cost Estimation for Major Equipment Used for Shift System in Case 1

4.11 Sizing and Cost Estimation for Major Equipment Used for $\mathrm{CO}_{2}$ Removal in Glycol Process in Case 1.

5.1 Material Flows for Oxygen-Blown Base Case and Case 2 ......................................... 65

5.2 Heat Recovery and Allocation for Gas Turbine/Membrane Process in Case 2 ............. 66

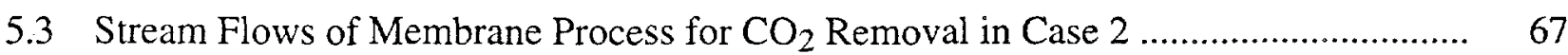

5.4 Descriptions of Streams of Membrane Process for $\mathrm{CO}_{2}$ Removal in Case 2 ................ 69

5.5 Power Output, Plant Power Use, and Net Power Output for Base Case and Case 2 Gas Turbine/Membrane Process 


\section{Tables (Cont.)}

5.6 Overall Power Recovery and Production for Three Gas Turbine Cases.

5.7 Sizing and Cost Estimation for Major Equipment Used for $\mathrm{H}_{2} \mathrm{~S}$ Removal in Glycol Process in Case 2 .

5.8 Sizing and Cost Estimation for Major Equipment Used for Shift System in Case 2

5.9 Sizing and Cost Estimation for Major Equipment Used for $\mathrm{CO}_{2}$ Removal in Membrane Process in Case 2

6.1 Material Flows for Oxygen-Blown Base Case and Case 3

6.2 Stream Flows of Chilled Methanol Process for $\mathrm{H}_{2} \mathrm{~S}$ Removal in Case 3

6.3 Description of Streams of Chilled Methanol Process for $\mathrm{H}_{2} \mathrm{~S}$ Removal in Case 3 .....

6.4 Stream Flows of Molten Carbonate Fuel Cell System in Case 3.

6.5 Descriptions of Streams of Fuel Cell System in Case 3

101

6.6 Stream Flows of Glycol System for $\mathrm{CO}_{2}$ Removal in Case 3

109

6.7 Descriptions of Streams of Glycol Process for $\mathrm{CO}_{2}$ Removal in Case 3 .

6.8 Power Output, Plant Power Use, and Net Power Output for Base Case and Case 3 Fuel Cell/Glycol Process

6.9 Sizing and Cost Estimation for Major Equipment Used for $\mathrm{H}_{2} \mathrm{~S}$ Removal in Chilled Methanol Process in Case 3

6.10 Sizing and Cost Estimation for Major Equipment Used for Fuel Cell System in Case 3 .

6.11 Sizing and Cost Estimation for Major Equipment Used for $\mathrm{CO}_{2}$ Removal in Glycol Process in Case 3.

7.1 Material Flows for Oxygen-Blown Base Case and Case 4

7.2 Stream Flows of Molten Carbonate Fuel Cell System in Case 4

7.3 Stream Flows of Membrane Process for $\mathrm{CO}_{2}$ Removal in Case 4

7.4 Descriptions of Streams of Membrane Process for $\mathrm{CO}_{2}$ Removal in Case 4 


\section{Tables (Cont.)}

7.5 Power Output, Plant Power Use, and Net Power Output for Base Case and Case 4 Fuel Cell/Membrane Process

7.6 Sizing and Cost Estimation for Major Equipment Used for Fuel Cell System in Case 4

7.7 Sizing and Cost Estimation for Major Equipment Used for $\mathrm{CO}_{2}$ Removal in Membrane Process in Case 4

9.1 Energy Consumption and $\mathrm{CO}_{2}$ Emissions for Oxygen-Blown Base Case:

KRW IGCC with No $\mathrm{CO}_{2}$ Recovery

9.2 Energy Consumption and $\mathrm{CO}_{2}$ Emissions for Air-Blown Base Case:

$\mathrm{KRW}$ IGCC with $\mathrm{No}_{2} \mathrm{CO}_{2}$ Recovery

156

9.3 Energy Consumption and $\mathrm{CO}_{2}$ Emissions for Case 1: Oxygen-Blown KRW IGCC with Glycol $\mathrm{CO}_{2}$ and $\mathrm{H}_{2} \mathrm{~S}$ Recovery and Gas Turbine Topping Cycle

9.4 Energy Consumption and $\mathrm{CO}_{2}$ Emissions for Case 2: Oxygen-Blown KRW IGCC with Membrane $\mathrm{CO}_{2}$ Recovery, Glycol $\mathrm{H}_{2} \mathrm{~S}$ Recovery, and Gas Turbine Topping Cycle

9.5 Energy Consumption and $\mathrm{CO}_{2}$ Emissions for Case 3: Oxygen-Blown KRW IGCC with Glycol $\mathrm{CO}_{2}$ Recovery, Methanol $\mathrm{H}_{2} \mathrm{~S}$ Recovery, and Fuel Cell Topping Cycle

9.6 Energy Consumption and $\mathrm{CO}_{2}$ Emissions for Case 4: Oxygen-Blown KRW IGCC with Membrane $\mathrm{CO}_{2}$ Recovery, Methanol $\mathrm{H}_{2} \mathrm{~S}$ Recovery, and Fuel Cell Topping Cycle

9.7 Energy Consumption and $\mathrm{CO}_{2}$ Emissions for Optimal Air-Blown Case:

KRW IGCC with Glycol $\mathrm{CO}_{2}$ Recovery, In-Bed $\mathrm{H}_{2} \mathrm{~S}$ Recovery, and

Gas Turbine Topping Cycle.

9.8 Capital Costs for Air-Blown and Oxygen-Blown Base Cases

with $\mathrm{No} \mathrm{CO}_{2}$ Recovery

9.9 Capital Costs for Case 1: Oxygen-Blown $\mathrm{KRW}$ IGCC with Glycol $\mathrm{CO}_{2}$ and $\mathrm{H}_{2} \mathrm{~S}$ Recovery and Gas Turbine Topping Cycle

9.10 Capital Costs for Case 2: Oxygen-Blown KRW IGCC with Membrane $\mathrm{CO}_{2}$ Recovery, Glycol $\mathrm{H}_{2} \mathrm{~S}$ Recovery, and Gas Turbine Topping Cycle

9.11 Capital Costs for Case 3: Oxygen-Blown KRW IGCC with Glycol $\mathrm{CO}_{2}$ Recovery, Methanol $\mathrm{H}_{2} \mathrm{~S}$ Recovery, and Fuel Cell Topping Cycle 


\section{Tables (Cont.)}

9.12 Capital Costs for Case 4: Oxygen-Blown KRW IGCC with Membrane $\mathrm{CO}_{2}$ Recovery, Methanol $\mathrm{H}_{2} \mathrm{~S}$ Recovery, and Fuel Cell Topping Cycle

9.13 Capital Costs for Optimal Air-Blown Case: KRW IGCC with Glycol $\mathrm{CO}_{2}$ Recovery, In-Bed $\mathrm{H}_{2} \mathrm{~S}$ Recovery, and Gas Turbine Topping Cycle

9.14 Operating Costs for Oxygen-Blown Base Case: KRW IGCC with No $\mathrm{CO}_{2}$ Recovery

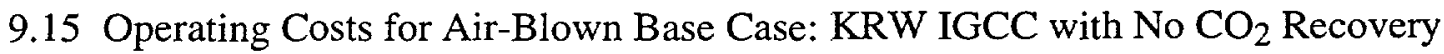

9.16 Operating Costs for Case 1: Oxygen-Blown KRW IGCC with Glycol $\mathrm{CO}_{2}$ and $\mathrm{H}_{2} \mathrm{~S}$ Recovery and Gas Turbine Topping Cycle

9.17 Operating Costs for Case 2: Oxygen-Blown KRW IGCC with Membrane $\mathrm{CO}_{2}$ Recovery, Glycol $\mathrm{H}_{2} \mathrm{~S}$ Recovery, and Gas Turbine Topping Cycle

9.18 Operating Costs for Case 3: Oxygen-Blown KRW IGCC with Glycol $\mathrm{CO}_{2}$ Recovery, Methanol $\mathrm{H}_{2} \mathrm{~S}$ Recovery, and Fuel Cell Topping Cycle

9.19 Operating Costs for Case 4: Oxygen-Blown KRW IGCC with Membrane $\mathrm{CO}_{2}$ Recovery, Methanol $\mathrm{H}_{2} \mathrm{~S}$ Recovery, and Fuel Cell Topping Cycle

9.20 Operating Costs for Optimal Air-Blown Case: KRW IGCC with Glycol $\mathrm{CO}_{2}$ Recovery, In-Bed $\mathrm{H}_{2} \mathrm{~S}$ Recovery, and Gas Turbine Topping Cycle.

9.21 Summary of Comparative Costs of IGCC Systems 


\section{Acknowledgment}

The authors wish to gratefully acknowledge the support and guidance provided by Dr. Richard A. Johnson of the Morgantown Energy Technology Center, who is the project officer for this research. 


\title{
KRW Oxygen-Blown Gasification Combined Cycle: Carbon Dioxide Recovery, Transport, and Disposal
}

\author{
by
}

R.D. Doctor, J.C. Molburg, and P.R. Thimmapuram

\begin{abstract}
The objective of the project is to develop engineering evaluations of technologies for the capture, use, and disposal of carbon dioxide $\left(\mathrm{CO}_{2}\right)$. This project emphasizes $\mathrm{CO}_{2}$-capture technologies combined with integrated gasification combined-cycle (IGCC) power systems. Complementary evaluations address $\mathrm{CO}_{2}$ transportation, $\mathrm{CO}_{2}$ use, and options for the long-term sequestration of unused $\mathrm{CO}_{2}$. Commercially available $\mathrm{CO}_{2}$-capture technology is providing a performance and economic baseline against which to compare innovative technologies. The intent is to provide the $\mathrm{CO}_{2}$ budget, or an "equivalent $\mathrm{CO}_{2}$ " budget, associated with each of the individual energy-cycle steps, in addition to process design capital and operating costs. The value used for the "equivalent $\mathrm{CO}_{2}$ " budget is $1 \mathrm{~kg}$ of $\mathrm{CO}_{2}$ per kilowatt-hour (electric). The base case is a 458-MW (gross generation) IGCC system that uses an oxygen-blown KelloggRust-Westinghouse agglomerating fluidized-bed gasifier, Illinois No. 6 bituminous coal feed, and low-pressure glycol sulfur removal followed by Claus/SCOT treatment to produce a saleable product. Mining, feed preparation, and conversion result in a net electric power production for the entire energy cycle of $411 \mathrm{MW}$, with a $\mathrm{CO}_{2}$ release rate of $0.801 \mathrm{~kg} / \mathrm{kWh}$. For comparison, in two cases, the gasifier output was taken through water-gas shift and then to lowpressure glycol $\mathrm{H}_{2} \mathrm{~S}$ recovery, followed by either low-pressure glycol or membrane $\mathrm{CO}_{2}$ recovery and then by a combustion turbine being fed a highhydrogen-content fuel. Two additional cases employed chilled methanol for $\mathrm{H}_{2} \mathrm{~S}$ recovery and a fuel cell as the topping cycle with no shift stages. From the IGCC plant, a $500-\mathrm{km}$ pipeline took the $\mathrm{CO}_{2}$ to geological sequestering. For the optimal $\mathrm{CO}_{2}$ recovery case, the net electric power production was reduced by $37.6 \mathrm{MW}$ from the base case, with a $\mathrm{CO}_{2}$ release rate of $0.277 \mathrm{~kg} / \mathrm{kWhe}$ (when makeup power was considered). In a comparison of air-blown and oxygen-blown $\mathrm{CO}_{2}$ release base cases, the cost of electricity for the air-blown IGCC was $56.86 \mathrm{mills} / \mathrm{kWh}$, and the cost for oxygen-blown IGCC was $58.29 \mathrm{mills} / \mathrm{kWh}$. For the optimal cases employing glycol $\mathrm{CO}_{2}$ recovery, there was no clear advantage; the cost for air-blown IGCC was 95.48 mills $/ \mathrm{kWh}$, and the cost for the $\mathrm{O}_{2}$-blown case was slightly lower, at 94.55 mills/kWh.
\end{abstract}




\section{Summary}

\section{S.1 Background}

Increasing atmospheric concentrations of carbon dioxide $\left(\mathrm{CO}_{2}\right)$ have the potential to cause significant climate-related impacts on ecosystems, food production, and economic development, as outlined in the U.S. Climate Change Action Plan (Clinton 1993). Because of these concerns, policies to limit $\mathrm{CO}_{2}$ emissions are being explored by the United States and other signatories to the Framework Convention on Climate Change put forward at the June 1992 Rio de Janeiro Earth Summit.

For example, Norway has imposed a carbon tax $\left(\$ 50 /\right.$ metric ton of $\left.\mathrm{CO}_{2}\right)$. As a result, Statoil (Trondheim, Norway) has submitted an engineering proposal for the disposal of $\mathrm{CO}_{2}$ recovered during natural gas production (Smith 1994). The $\mathrm{CO}_{2}$ sequestering is to be in an aquifer located $800 \mathrm{~m}$ below the sea bed $250 \mathrm{~km}$ offshore; as of the date of this publication, however, there has been no final decision to move forward. In Japan, work on disposing of $\mathrm{CO}_{2}$ in the ocean continues. At the same time, now that this work has reached a more serious stage, there are some significant concerns being expressed by the Japanese government, which would rather see the $\mathrm{CO}_{2}$ utilized. At present, the only signatories to the Rio Convention on Climate Change that are meeting the goal of maintaining $1990 \mathrm{CO}_{2}$ release levels are the United Kingdom, Denmark, and Germany (Stone 1994).

In October 1994, the U.S. Department of Energy (DOE) released greenhouse gas reporting guidelines, but for the present, participation is voluntary. The U.S. actions to stabilize $\mathrm{CO}_{2}$ may include mandatory conservation - something like establishing $\mathrm{Btu} / \mathrm{kWh}$ efficiency ratings for electric power plants similar to the fleet fuel efficiency standards for automobiles. Other options may include taking strong energy conservation measures, switching from coal to natural gas for electric power generation, capturing and sequestering $\mathrm{CO}_{2}$, or substituting nonfossil energy sources for fossil fuel combustion. Discussion of the issues has drawn considerable interest in power generating systems that minimize the production of $\mathrm{CO}_{2}$ and are amenable to $\mathrm{CO}_{2}$ capture. In the event that natural gas would no longer be widely available at low prices, integrated gasification combined-cycle (IGCC) systems would be an attractive emerging electric power generating technology option because they provide high energyconversion efficiency when current technology is used. They also offer the prospect of even higher efficiencies if higher-temperature turbines and hot-gas cleanup systems are developed. In addition, they have demonstrated very low emission levels for sulfur and nitrogen species. Finally, IGCC plants produce flue-gas streams with concentrated $\mathrm{CO}_{2}$ and high levels of $\mathrm{CO}$, which can be easily converted to $\mathrm{CO}_{2}$ if the recovery and sequestering of $\mathrm{CO}_{2}$ are mandated in the future.

The project objective is to develop engineering evaluations of technologies used to capture, use, and dispose of $\mathrm{CO}_{2}$ when combined with oxygen $\left(\mathrm{O}_{2}\right)$-blown Kellogg-RustWestinghouse (KRW) IGCC power systems. This study is an extension of earlier work done for the Morgantown Energy Technology Center (METC) that considered these questions for airblown KRW IGCC power systems (Doctor et al. 1994). 


\section{S.2 Overview of Energy Cycle}

The energy system definition for this study extends from the coal mine to the final geological repository for the $\mathrm{CO}_{2}$, as shown in Figure S.1. The location of the IGCC plant is specified as the midwestern United States, and this study assumes it is $160 \mathrm{~km}$ by rail from the Old Ben No. 26 mine in Sesser, Illinois. Details of the IGCC portion of the system are taken from an Electric Power Research Institute (EPRI) report (Gallaspy 1990a), which describes an electric power station using an $\mathrm{O}_{2}$-blown $\mathrm{KRW}$ gasifier, while a follow-up METC report (Gallaspy 1990b) describes a plant using an air-blown KRW gasifier with in-bed sulfur removal. In each case studied, the $\mathrm{CO}_{2}$ recovery technologies have been integrated into that plant design as much as possible to limit efficiency losses. For each part of the energy system, $\mathrm{CO}_{2}$ emissions have been either computed directly from process stream compositions or calculated from energy consumption on the basis of a " $\mathrm{CO}_{2}$ equivalence" of $1 \mathrm{~kg}$ of $\mathrm{CO}_{2}$ per kilowatt-hour (electric) ( $\mathrm{kWhe}$ ). In this way, a total $\mathrm{CO}_{2}$ budget for the system can be derived and compared with a total $\mathrm{CO}_{2}$ budget for other options, thereby taking into account effects outside the immediate plant boundary.

\section{S.3 Mining, Preparation, and Transportation of Raw Materials}

All seven cases presented here were adjusted to be on a consistent basis of 4,110 tons/d (stream day) of Illinois No. 6 coal from the Old Ben No. 26 mine. This bituminous $2.5 \%$-sulfur coal contains $9.7 \%$ ash. The underground mine is associated with a coal preparation plant. The assumption is that the IGCC power plant is $160 \mathrm{~km}$ from the mine and the coal is shipped by rail on a unit train. The impact of coal mining and shipment on the energy budget is $2.41 \mathrm{MW}$ of power use and $2,879 \mathrm{~kg} / \mathrm{h}$ of $\mathrm{CO}_{2}$ emissions.

Limestone is used for in-bed sulfur capture in the two air-blown gasifier cases. It is assumed that the limestone is extracted from a quarry about $160 \mathrm{~km}$ from the plant and transported by rail to the plant site. The impact of limestone mining and shipment on the energy budget is $0.27 \mathrm{MW}$ of power use and $406 \mathrm{~kg} / \mathrm{h}$ of $\mathrm{CO}_{2}$ emissions.

\section{S.4 Handling of Coal and Limestone}

The coal preparation system for the $\mathrm{O}_{2}$-blown IGCC plant includes equipment for unloading the coal from the unit train, passing it through magnetic separators, and then conveying it to a hammermill. From there, the coal is conveyed to storage silos from which it is recovered in a fluidized stream for use in the gasifier. The coal is not dried for the $\mathrm{O}_{2}$-blown cases. The impact of coal preparation on the energy budget is $0.85 \mathrm{MW}$ of power use and no $\mathrm{CO}_{2}$ emissions (these will be combined with the overall emissions from the IGCC plant). Drying the coal was not considered for this case. 


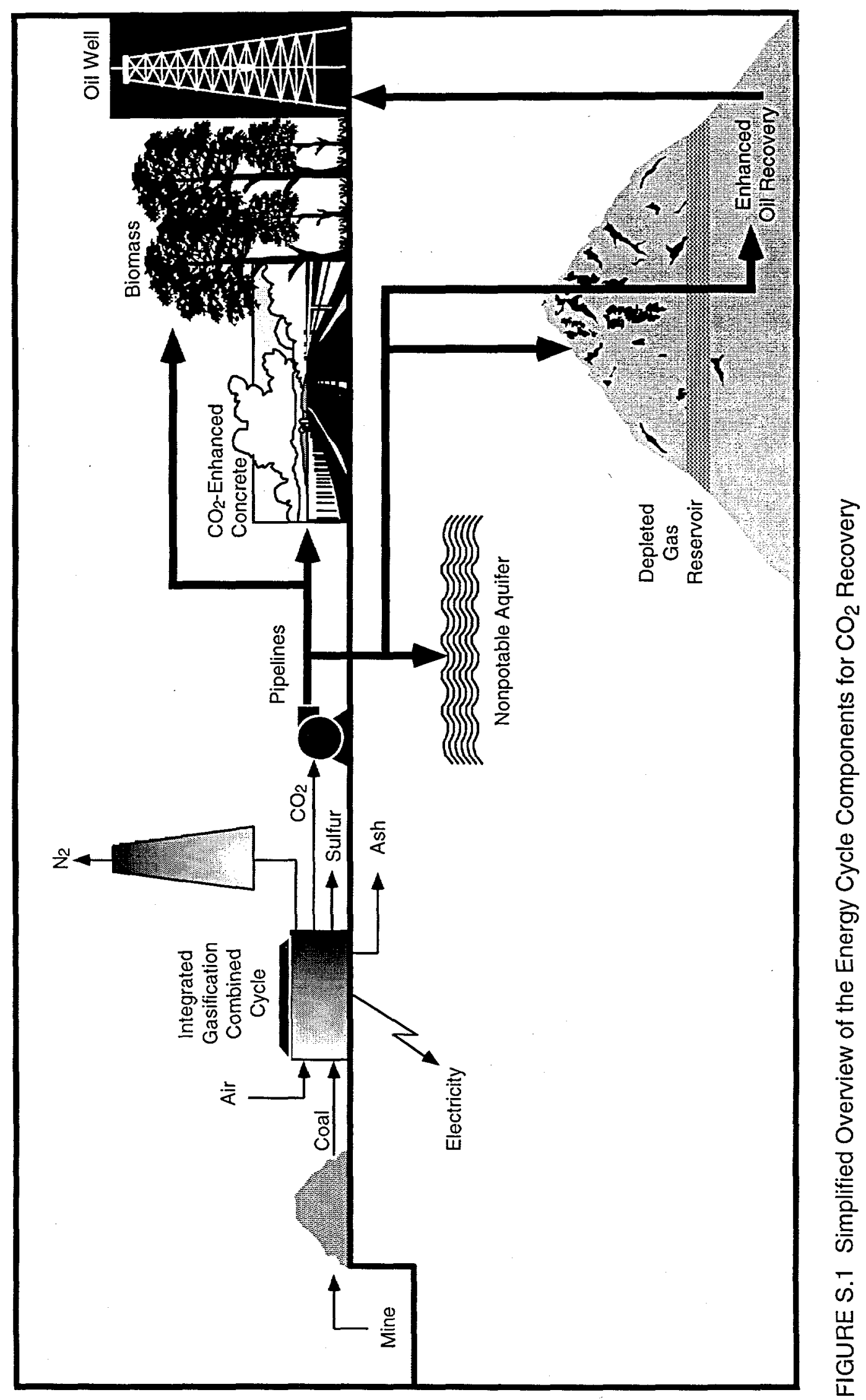


By way of contrast, the coal preparation system for the air-blown IGCC plant includes equipment for unloading the coal from the unit train, passing it through magnetic separators, and then conveying it to silos for 14-h storage. The coal is crushed and dried in a series of three fluidized-bed roller mills. The heat for drying is provided by the hot $\left(760^{\circ} \mathrm{C}\right)$ flue gas from the IGCC sulfator process. This drying results in a significant amount of $\mathrm{CO}_{2}$ being emitted from the energy cycle that is not reclaimed and presents a possible opportunity for further reduction. The coal is then held in a bunker for $2 \mathrm{~h}$, from which it is pneumatically conveyed to surge bins ahead of the gasifier lockhoppers. The sulfator emits $11,374 \mathrm{~kg} / \mathrm{h}$ of $\mathrm{CO}_{2}$. Limestone is crushed in two pulverizers and then pneumatically conveyed to a $24-\mathrm{h}$ storage silo and a 2 -h storage bunker before being mixed with the coal in the gasifier surge bins. Energy use for coal and limestone preparation is $3.49 \mathrm{MW}$.

\section{S.5 Base Cases for Integrated Gasification Combined Cycle}

\section{S.5.1 Gasifier Island}

The $\mathrm{O}_{2}$-blown base case employs an air-separation plant producing 2,100 tons/d of $95 \%$ oxygen from a commercial package designed by Air Products. The KRW process is an $\mathrm{O}_{2}$-blown, dry-ash, agglomerating, fluidized-bed process. A simplified schematic for this process appears in Figure S.2. Three parallel gasifier trains operating at $450 \mathrm{lb} / \mathrm{in}^{2}$ gauge (psig) and $1,850^{\circ} \mathrm{F}$ are included in the design. Following gasification, cyclones recover $95 \%$ of the fines; gas cooling and high-efficiency particulate removal follow. For the base case, glycol $\mathrm{H}_{2} \mathrm{~S}$ recovery provides a feed to a conventional Claus tail-gas cleanup system. Hence, the significant differences between the $\mathrm{O}_{2}$-blown and air-blown cases are that the $\mathrm{O}_{2}$-blown cases cool the product gas for sulfur cleanup and produce a sulfur product for the market, while the air-blown cases employ hot-gas cleanup and produce a landfill product. The impact of the gasifier island operation on the energy budget is $36.82 \mathrm{MW}$ of power use and $6,153 \mathrm{~kg} / \mathrm{h}$ of $\mathrm{CO}_{2}$ emissions for the $\mathrm{O}_{2}$-blown base case.

The air-blown base case uses in-bed sulfur removal. A simplified schematic for this process appears in Figure S.3. The system includes two heavy-duty industrial gas turbines $\left(2,300^{\circ} \mathrm{F}\right.$ firing temperature) coupled with a reheat steam-turbine bottoming cycle. Spent limestone and ash from the gasifier are oxidized in an external sulfator before disposal. The sulfator flue gas is taken to the coal preparation operation for drying coal and not integrated into the later $\mathrm{CO}_{2}$ recovery operation. The hot-gas cleanup system for particulate matter consists of a cyclone followed by a ceramic-candle-type filter. Solids collected are sent to the external sulfator before disposal. Inlet gas temperatures are maintained at approximately $1,000^{\circ} \mathrm{F}$. Supplemental hot-gas desulfurization is accomplished in a fixed-bed zinc-ferrite system. Off-gas from the regeneration of this polishing step is recycled to the gasifier for in-bed sulfur capture. The impact of the gasifier island operation on the energy budget is $20.12 \mathrm{MW}$ of power use and $137 \mathrm{~kg} / \mathrm{h}$ of $\mathrm{CO}_{2}$ emissions for the air-blown base case. 


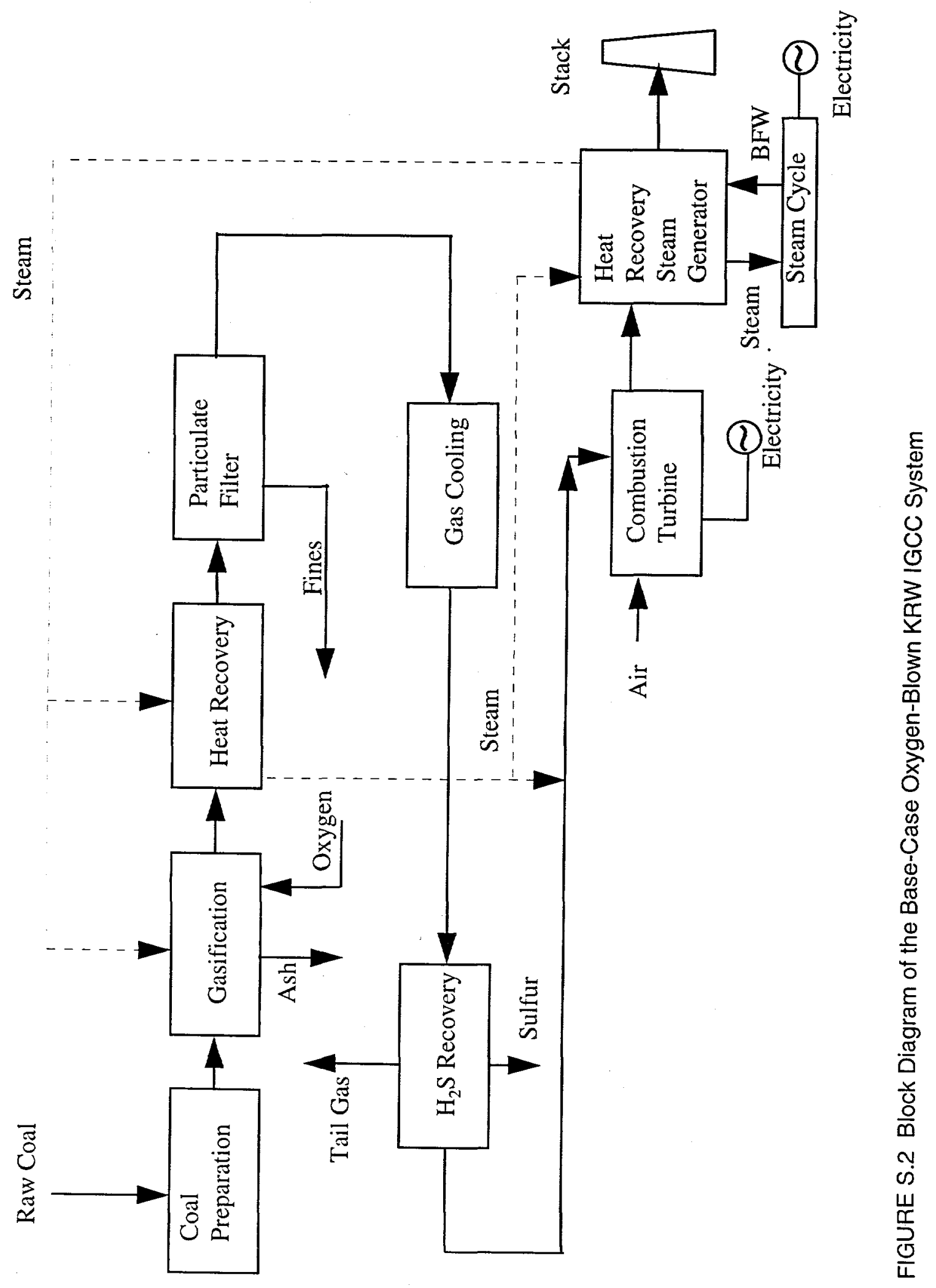




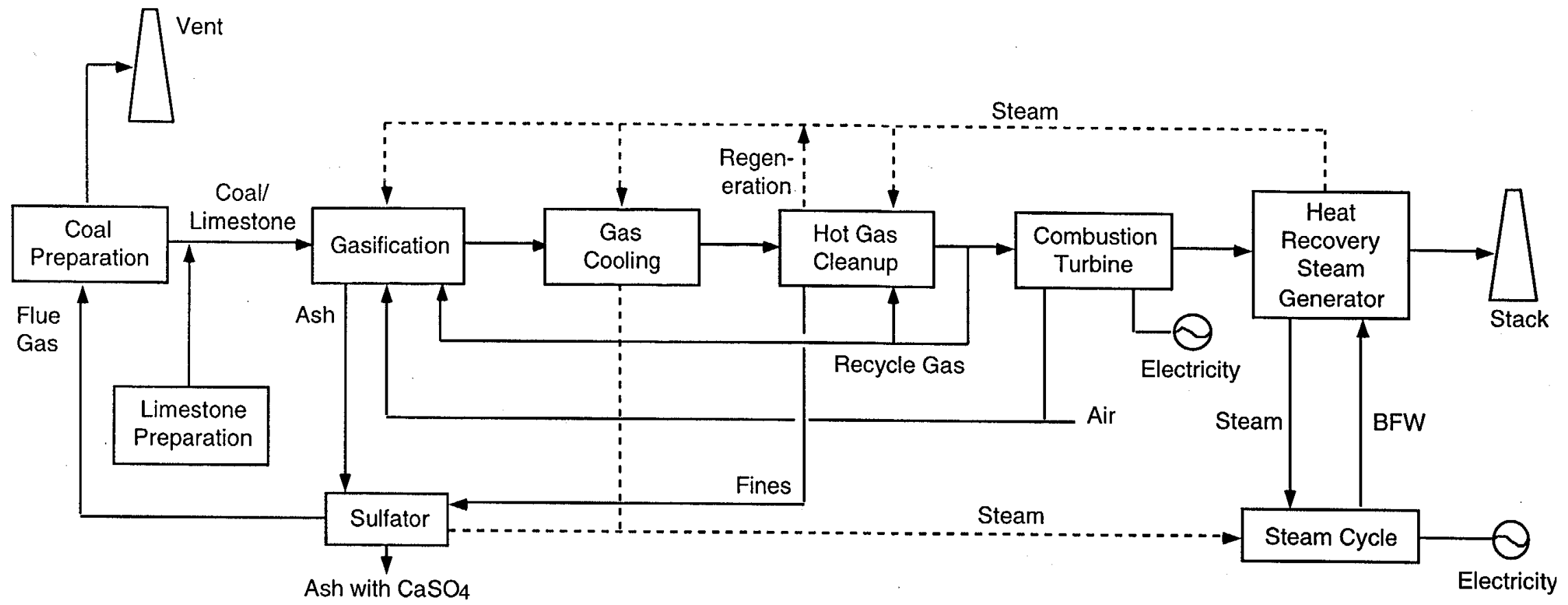

FIGURE S.3 Block Diagram of the Base-Case Air-Blown KRW IGCC System 


\section{S.5.2 Power Island}

Both the $\mathrm{O}_{2}$-blown and air-blown base cases employ a turbine topping cycle and a steam bottoming cycle based on two heavy-duty GE MS701F industrial gas turbines with a $2,300^{\circ} \mathrm{F}$ firing temperature. The impact on the energy budget of the power island operation is $7.02 \mathrm{MW}$ of power use for the $\mathrm{O}_{2}$-blown base case and $10.58 \mathrm{MW}$ of power use for the air-blown base case. For the $\mathrm{O}_{2}$-blown base case, gross power generation is $458.20 \mathrm{MW}$, with a net generation of $413.50 \mathrm{MW}$; for the air-blown base case, gross power generation is $479.63 \mathrm{MW}$, with a net power generation of $445.44 \mathrm{MW}$.

\section{S.6 Integrated Gasification Combined Cycle with $\mathrm{CO}_{2}$ Recovery}

Several changes were made to the base-case IGCC plant to incorporate $\mathrm{CO}_{2}$ recovery. For the turbine topping-cycle studies (Cases 1 and 2), these changes entailed processing the cleaned fuel gas through a "shift" reaction to convert the $\mathrm{CO}$ to $\mathrm{CO}_{2}$, recovering the $\mathrm{CO}_{2}$, and then combusting the low- $\mathrm{CO}_{2}$ fuel gas in a modified turbine/steam cycle to produce electricity. Gas cleaning and sulfator performance were considered to be unaffected by these changes. In contrast, the fuel cell topping-cycle studies (Cases 3 and 4) required a highly cleaned gasifier without use of the water-gas shift reaction to be used by the fuel cells. A block diagram of the $\mathrm{O}_{2}$-blown IGCC system with $\mathrm{CO}_{2}$ recovery appears in Figure S.4, while the air-blown system with $\mathrm{CO}_{2}$ recovery appears in Figure S.5.

The fuel gas from the KRW process is high in $\mathrm{CO}$. Conversion of the $\mathrm{CO}$ to $\mathrm{CO}_{2}$ in the combustion process would result in substantial dilution of the resulting $\mathrm{CO}_{2}$ with nitrogen from the combustion air and with water from the combustion reaction. If the $\mathrm{CO}_{2}$ is removed before combustion, a substantial savings in the cost of the $\mathrm{CO}_{2}$ recovery system is possible because of reduced vessel size and solvent flow rate. The $\mathrm{CO}$ in the fuel gas must first be converted to $\mathrm{CO}_{2}$ by the shift reaction:

$$
\mathrm{CO}+\mathrm{H}_{2} \mathrm{O}==\mathrm{CO}_{2}+\mathrm{H}_{2} \text {. }
$$

The resulting $\mathrm{CO}_{2}$ can then recovered, leaving a hydrogen-rich fuel for use in the gas turbine. The shift reaction is commonly accomplished in a catalyst-packed tubular reactor that uses a relatively low-cost iron-oxide catalyst. High $\mathrm{CO}_{2}$ recovery is best achieved by staged reactors that allow for cooling between stages; hence, a two-stage system configured to achieve $95 \%$ conversion of $\mathrm{CO}$ to $\mathrm{CO}_{2}$ was found to be optimal.

Commercial $\mathrm{CO}_{2}$-removal technologies all involve cooling or refrigerating the gas stream, with an attendant loss of thermal efficiency. To minimize the loss, the heat removed during cooling must be recovered and integrated into the system. Several options for this integration were evaluated, including steam generation alone, fuel-gas preheating with supplemental steam generation, and fuel-gas saturation and preheating. In the latter case, moisture condensed from the fuel gas before $\mathrm{CO}_{2}$ recovery is injected into the clean fuel-gas stream as it is heated by recovered heat following $\mathrm{CO}_{2}$ removal. This option allows additional 


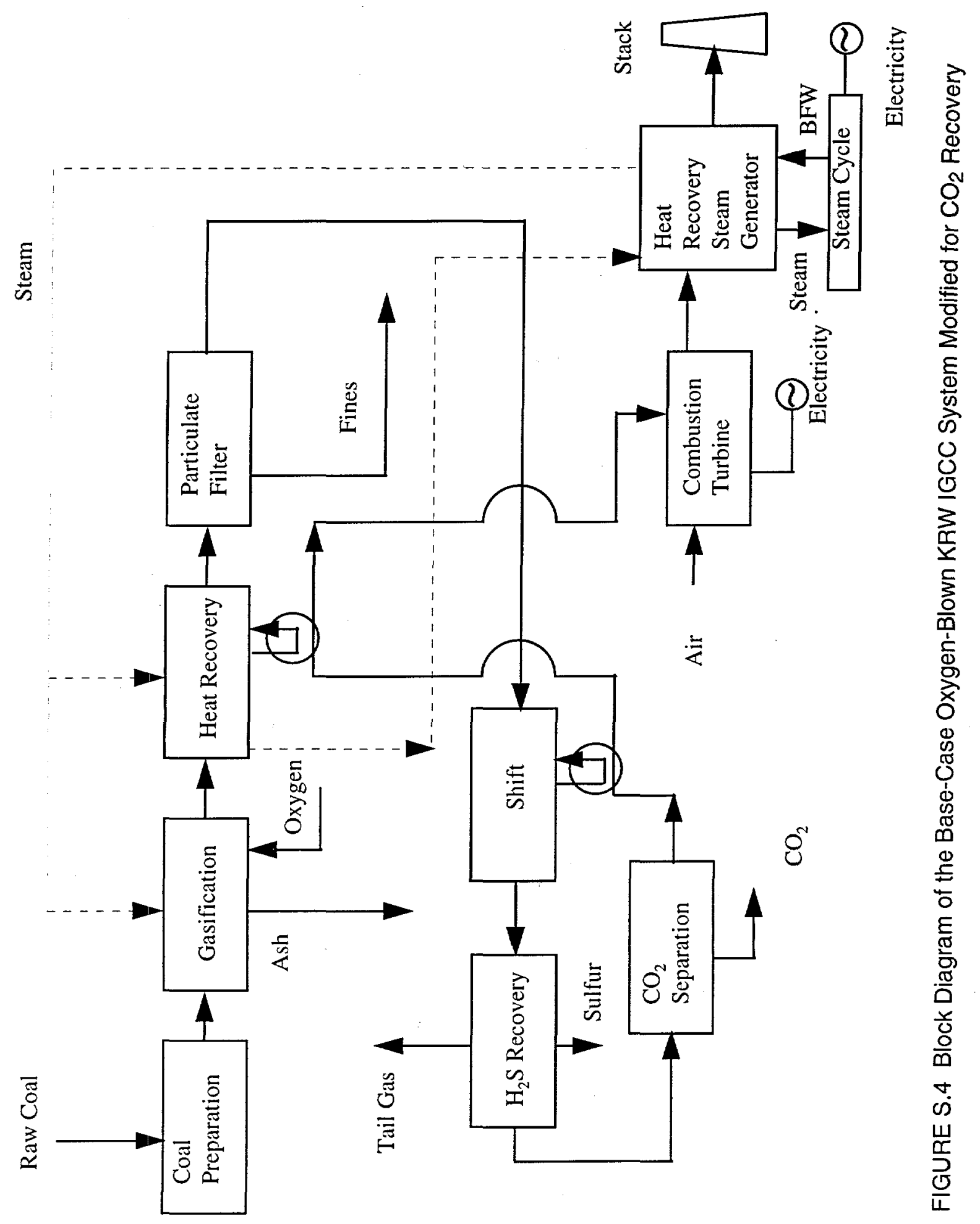




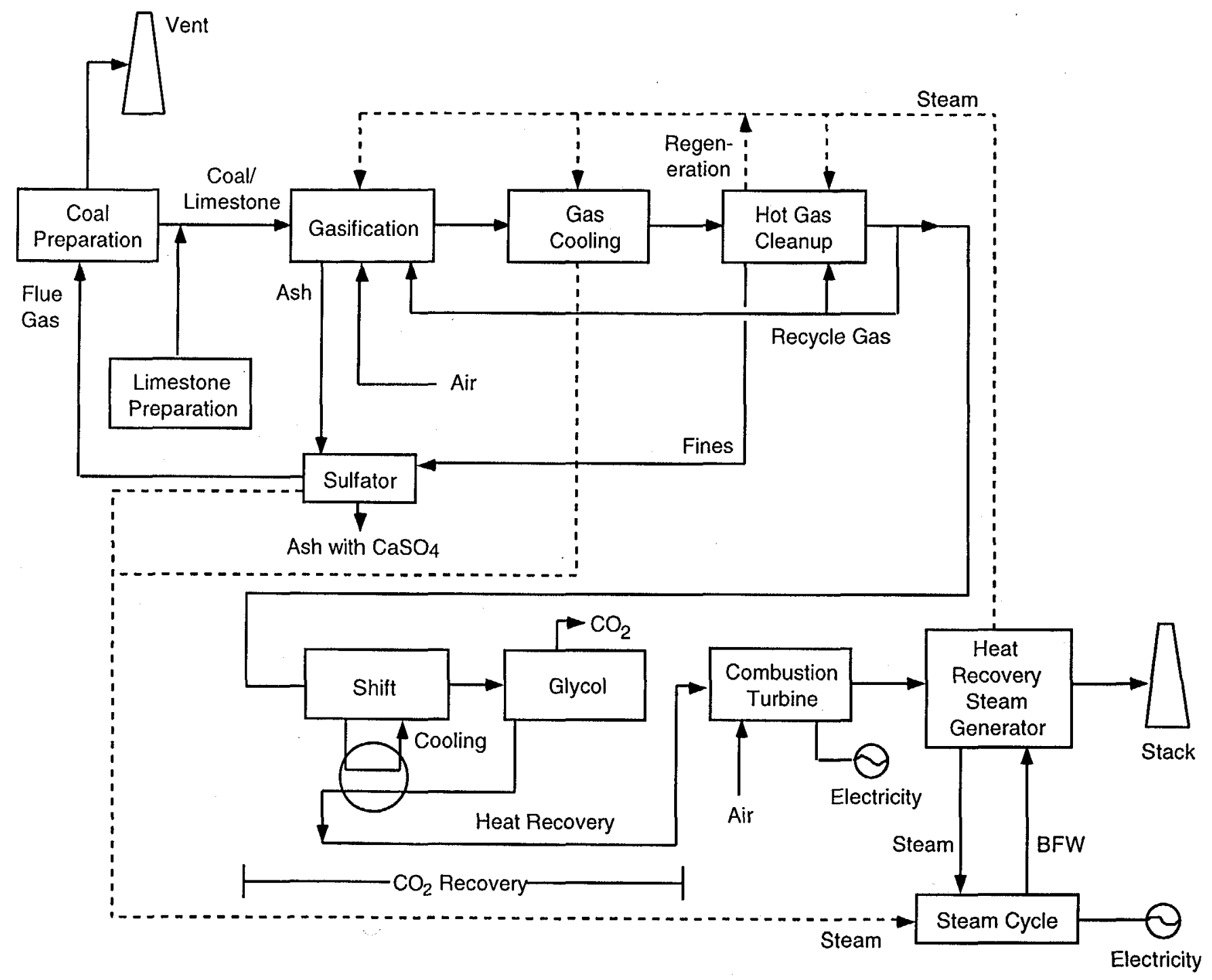

FIGURE S.5 Block Diagram of the Base-Case Air-Blown KRW IGCC System Modified for $\mathrm{CO}_{2}$ Recovery 
heat to be absorbed before combustion and increases the mass flow rate through the gas turbine. The balance of the thermal energy is used in the heat recovery steam generator for feedwater heating and steam generation.

Commercial $\mathrm{CO}_{2}$-recovery processes operate by absorption of the $\mathrm{CO}_{2}$ in a liquid solvent and subsequent regeneration of the solvent to release the $\mathrm{CO}_{2}$. The temperature of absorption is solvent-specific. In general, however, the solvents have low boiling points so that substantial cooling of the synthesis gas is required, as noted above. Furthermore, lower temperatures favor absorption, thereby reducing the necessary solvent flow rate. This situation implies a need for further cooling or refrigeration of the solvent, with additional energy losses. The regeneration of the solvent is also energy-intensive for most processes, since it is usually accomplished by flashing (pressure reduction) and/or heating. If flashing is employed, repressurization of the solvent is required. Heating is generally accomplished by the extraction of steam from the steam cycle.

In addition to supplying data on an oxygen-blown base case and an air-blown base case (both without $\mathrm{CO}_{2}$ recovery), this study evaluates five $\mathrm{CO}_{2}$ recovery power cycles: four oxygenblown cases and the optimal air-blown case discussed in our previous study, ANL/ESD-24 (Doctor et al. 1994).

$\begin{array}{llllll} & \text { Case 1 } & \text { Case 2 } & \text { Case 3 } & \text { Case 4 } & \text { ANL/ESD-24 } \\ \text { Gasifier oxidant } & \text { Oxygen } & \text { Oxygen } & \text { Oxygen } & \text { Oxygen } & \text { Air } \\ \mathrm{H}_{2} \mathrm{~S} \text { recovery } & \text { Glycol } & \text { Glycol } & \text { Methanol } & \text { Methanol } & \text { In-bed/ZnTi } \\ \mathrm{CO}_{2} \text { recovery } & \text { Glycol } & \text { Membrane } & \text { Glycol } & \text { Membrane } & \text { Glycol } \\ \text { Topping cycle } & \text { Turbine } & \text { Turbine } & \text { Fuel cell } & \text { Fuel cell } & \text { Turbine } \\ \text { Bottoming cycle } & \text { Steam } & \text { Steam } & \text { Steam } & \text { Steam } & \text { Steam }\end{array}$

For the optimal $\mathrm{O}_{2}$-blown $\mathrm{CO}_{2}$ recovery case (Case 1), the net electric power production was reduced by $37.6 \mathrm{MW}$ from the base case, with a $0.277-\mathrm{kg} / \mathrm{kWhe} \mathrm{CO}_{2}$ release rate (when makeup power was considered). The low-pressure glycol system, which does not require compression of the synthesis gas before absorption, appears to be the best system studied.

\section{S.7 Pipeline Transport of $\mathrm{CO}_{2}$}

Once the $\mathrm{CO}_{2}$ has been recovered from the fuel-gas stream, its transportation, utilization, and/or disposal remain significant issues. In a previous study for METC (Doctor et al. 1994), the issues associated with the transport and sequestering of $\mathrm{CO}_{2}$ were considered in greater detail; they serve as the basis for this work. The $\mathrm{CO}_{2}$ represents a large-volume, relatively low-value by-product that cannot be sequestered in the same way as most coal-utilization wastes (i.e., by landfilling). Large volumes of recovered $\mathrm{CO}_{2}$ are likely to be moved by pipeline, and if sequestering were required, new pipelines would likely need to be constructed. In some cases, existing pipelines could be used, perhaps in a shared mode with other products. Costs for pipeline construction and use vary greatly on a regional basis within the United States. The recovered $\mathrm{CO}_{2}$ represents more than 3 million normal cubic meters per day of gas volume. It is 
assumed that the transport and sequestering process releases approximately $2 \%$ of the recovered $\mathrm{CO}_{2}$.

\section{S.8 Sequestering of $\mathrm{CO}_{2}$}

Proposals have been made to dispose of $\mathrm{CO}_{2}$ in the ocean depths. However, many questions of engineering and ecological concern associated with such options remain unanswered, and the earliest likely reservoir is a land-based geological repository (Hangebrauck et al. 1992). A portion of the $\mathrm{CO}_{2}$ can be used for enhanced oil recovery, which sequesters a portion of the $\mathrm{CO}_{2}$, or the $\mathrm{CO}_{2}$ can be completely sequestered in depleted gas/oil reservoirs and nonpotable aquifers. Both the availability of these zones and the technical and economic limits to their use need to be better characterized. Levelized costs have been prepared; they take into account that the power required for compression will rise throughout the life cycle of these sequestering reservoirs. The first reservoirs that would be used will, in fact, be capable of accepting all IGCC $\mathrm{CO}_{2}$ gas for a 30 -year period without requiring any additional compression costs for operation. The pipeline transport and sequestering process represents approximately 26 mills/kWh for the $\mathrm{CO}_{2}$-recovery cases.

\section{S.9 Energy Consumption and $\mathrm{CO}_{2}$ Emissions}

Data on the energy consumption and $\mathrm{CO}_{2}$ emissions for the $\mathrm{O}_{2}$-blown base case are provided in Table S.1. These can be compared with data on the optimal case that employs lowpressure glycol $\mathrm{CO}_{2}$ recovery and a turbine topping cycle (i.e., Case 1) provided in Table S.2.

\section{S.10 Economic Summary}

A comparison of the cost of electricity for the $\mathrm{CO}_{2}$ release base cases revealed that the cost for the air-blown IGCC was 58.29 mills $/ \mathrm{kWh}$, and the cost for the $\mathrm{O}_{2}$-blown case was $56.86 \mathrm{mills} / \mathrm{kWh}$ (Table S.3). There was no clear advantage for the optimal cases employing glycol $\mathrm{CO}_{2}$ recovery; the cost for the air-blown IGCC was $95.48 \mathrm{mills} / \mathrm{kWh}$, and the cost for the $\mathrm{O}_{2}$-blown case was slightly lower, at 94.55 mills $/ \mathrm{kWh}$.

\section{S.11 References for Summary}

Clinton, W.J., and A. Gore, Jr., 1993, The Climate Change Action Plan, Washington, D.C., Oct.

Doctor, R.D., et al., 1994, Gasification Combined Cycle: Carbon Dioxide Recovery, Transport, and Disposal, ANL/ESD-24, Argonne National Laboratory, Argonne, Ill., Sept.

Gallaspy, D.T., et al., 1990a, Southern Company Service's Study of a KRW-Based GCC Power Plant, EPRI GS-6876, Electric Power Research Institute, Palo Alto, Calif., July. 
Gallaspy, D.T., et al., 1990b, Assessment of Coal Gasification/Hot Gas Cleanup Based Advanced Gas Turbine Systems: Final Report, DOE/MC/26019.3004 (DE91002084), prepared by Southern Company Services, Inc., Birmingham, Ala., et al., for U.S. Department of Energy, Morgantown Energy Technology Center, Morgantown, W. Va., Dec.

Hangebrauck, R.P., et al., 1992, "Carbon Dioxide Sequestration," in Proceedings of the 1992 Greenhouse Gas Emissions and Mitigation Research Symposium, sponsored by the U.S. Environmental Protection Agency, Washington, D.C., Aug. 18-20.

Smith, A., 1994, "Norway Pioneers Large Scale $\mathrm{CO}_{2}$ Disposal in 1996," Greenhouse Issues, International Energy Agency, Glouchestershire, U.K., Aug.

Stone, R., 1994, "Most Nations Miss the Mark on Emission-Control Plans," Science 226(5193): 1939, Dec. 23. 
TABLE S. 1 Energy Consumption and $\mathrm{CO}_{2}$ Emissions for Oxygen-Blown Base Case with $\mathrm{No} \mathrm{CO}_{2}$ Recovery

Mining and Transport

Raw Coal in Mine

Coal Rail Transport

Subtotal

Electricity $\mathrm{CO} 2$ release

MW

$\mathrm{kg} / \mathrm{h}$

$-2.36 \quad 2,356$

$\begin{array}{ll}-0.05 & 523\end{array}$

$-2.41 \quad 2,879$

IGCC Power Plant

Coal Preparation

Gasifier Island

$-0.85$

Power Island

$-36.82$

6,153

Subtotal

.7 .02

320,387

$-44.70$

326,540

Power - Gas Turbine

298.80

Power - Steam Turbine

159.40

GROSS Power

458.20

NET Power

413.50

Pipeline/Sequester

0.00

0

Energy Cycle Power Use

.47 .11

NET Energy Cycle

411.09

329,419

$\mathrm{CO} 2$ emission rate/net cycle

Power use/ $\mathrm{CO} 2$ in reservoir

$0.801 \mathrm{~kg} \mathrm{CO} / \mathrm{kWh}$

N/A $\mathrm{kWh} / \mathrm{kg} \mathrm{CO} 2$ 
TABLE S.2 Energy Consumption and $\mathrm{CO}_{2}$ Emissions for Optimal Oxygen-Blown Case with $\mathrm{CO}_{2}$ Recovery: Case 1

Mining and Transport

Electricity $\mathrm{CO} 2$ release Raw Coal in Mine MW $\mathrm{kg} / \mathrm{h}$

Coal Rail Transport $-2.36 \quad 2,356$

Subtotal $-0.05$

IGCC Power Plant Coal Preparation Gasifier Island $-0.85$ $-36.82$

Power Island

$-7.02$

Glycol Circulation

$-5.80$

Glycol Refrigeration

$-4.50$

Power Recovery Turbines

3.40

$\mathrm{CO} 2$ Compression (to 2100psi)

$-17.30$

Subtotal

$-68.90$

66,485

Power - Gas Turbine

284.80

Power - Steam Turbine

161.60

GROSS Power

446.40

NET Power

377.50

Pipeline/Sequester

Pipeline $\mathrm{CO} 2$

Pipeline booster stations

260,055

$-1.64 \quad 1,637$

Geological reservoir (2\% loss)

$0.00 \quad-254,854$

Subtotal

$-1.64 \quad 6,839$

Energy Cycle Power Use

.72 .95

NET Energy Cycle

$373.45 \quad 76,202$

Derating from 02-Base Case

37.64

Make-up Power

$37.64 \quad 37,637$

TOTAL

$411.09 \quad 113,840$

$\mathrm{CO} 2$ emission rate/net cycle

$0.277 \mathrm{~kg} \mathrm{CO} 2 / \mathrm{kWh}$

Power use/ $\mathrm{CO} 2$ in reservoir

$0.148 \mathrm{kWh} / \mathrm{kg} \mathrm{CO} 2$ 
TABLE S.3 Summary of Comparative Costs of IGCC Systems

\section{Case}

Gasifier Oxidant

H2S Recovery

CO2 Recovery

Topping Cycle

Bottoming Cycle

Component

Base Plant Capilal

CO2 Control Capital

Total Plant Capital

Power Plant Annual Cost

Power Cost

Base Plant Power Cost

Pipcline Cost

Net Power Cost

Coal Energy Input

Gross Power Output

In Plant Power Use

Net Plant Output

Net Heat Rate

Thermal Efficiency - HHV

Out of Plant Power Use

Net Energy Cycle Power

Net Energy Cycle Heat Rate

Thermal Efliciency - HHV

Net Encrgy Cycle Power

$\mathrm{Net}$ Replacement [Added] Power

Net Grid Power

$\begin{array}{rr}\text { BASE } & \text { BASE } \\ \text { Oxygen } & \text { Air } \\ \text { Glycol } & \text { In-Bed/ZnTi } \\ \text { none } & \text { none } \\ \text { Turbine } & \text { Turbine } \\ \text { Steam } & \text { Steam }\end{array}$

Unit

$\$ / k W$

$\$ / k W$

$\$ / \mathbf{k W}$

$\$ K$

$\$ 1,332$
$\$()$
$\$ 1,332$
$\$ 137,253$

$\$ 144,212$

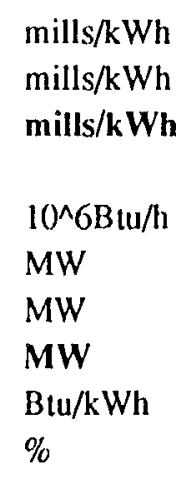

58.29

0
58.29

3839

458.20

44.70

413.50

9284

$36.78 \%$

\section{MW}

MW

Btu/kWh

$\%$

MW

MW

MW
2.41

411.09

9339

$36.56 \%$

411.09

0.00

411.09
Case \#1

Oxygen

Glycol

Glycol

Turbine

Stcam

$\$ 1,253$

$\$ 1,253$

\author{
$\$ 1,485$ \\ $\$ 202$ \\ $\$ 1,687$ \\ $\$ 203,238$
}

56.86

56.86

Case \#2

Membrane

$\$ 242,336$

$$
\begin{array}{r}
70.64 \\
23.91 \\
\mathbf{9 4 . 5 5} \\
\\
3839 \\
446.40 \\
68.90 \\
377.50 \\
10170 \\
33.58 \%
\end{array}
$$

3839

479.63

34.19

445.44

8618

$39.62 \%$

$$
4.18
$$

441.26

8700

$39.25 \%$

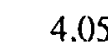

$\mathbf{3 7 3 . 4 5}$

10280

$33.21 \%$

441.26

(30.17)

411.09
373.45

37.64

411.09
Oxygen

Glycol

Turbine

Steam

$\$ 1,703$

$\$ 602$

$\$ 2,305$

101.62

27.35

128.97

Case\#3

Oxygen

Methanol

Glycol

Fuel Cell

Steam

$\$ 2,560$

$\$ 145$

$\$ 2,705$

$\$ 249,786$

3839

417.60

87.60

330.00)

11633

$29.35 \%$

3.87

326.13

11771

$29.01 \%$

326.13

84.96

411.09
102.45

26.53

128.98

3839

418.50

78.39

340.11

11288

$30.25 \%$

4.05

336.06

11424

$29.89 \%$

336.06

75.03

411.09
Case \#4 ESD-24/Glycol

Oxygen

Air

Methanol In-Bed/ZnTi

Membrane Glycol

Fuel Cell Turbine

Steam, Steam

$\begin{array}{rr}\$ 2,746 & \$ 1,487 \\ \$ 905 & \$ 246 \\ \mathbf{\$ 3 , 6 5 1} & \mathbf{\$ 1 , 7 3 3} \\ \$ 287,547 & \$ 204,288\end{array}$

$\begin{array}{rr}132.19 & 71.46 \\ 28.76 & 24.02 \\ 160.95 & 95.48\end{array}$

$3839 \quad 3839$

$413.20 \quad 460.88$

$99.40 \quad 85.11$

$313.80 \quad 375.77$

$12234 \quad 10216$

$27.91 \% \quad 33.42 \%$

$\begin{array}{rr}4.12 & 4.47 \\ 309.68 & 371.30 \\ 12397 & 10339 \\ 27.54 \% & 33.02 \%\end{array}$

$\begin{array}{rr}309.68 & 371.30 \\ 101.41 & 39.79 \\ 411.09 & 411.09\end{array}$




\section{Introduction}

\subsection{Background}

Argonne National Laboratory report ANL/ESD-24, Gasification Combined Cycle: Carbon Dioxide Recovery, Transport, and Disposal (Doctor et al. 1994), provides a comparison of carbon dioxide $\left(\mathrm{CO}_{2}\right)$ recovery options for an integrated gasification combined-cycle (IGCC) plant using an air-blown Kellogg-Rust-Westinghouse (KRW) gasifier that employs an in-bed

sorbent system for sulfur recovery. The comparison focuses on the relative energy penalty, capital investment, and $\mathrm{CO}_{2}$ reduction for five commercial $\mathrm{CO}_{2}$ recovery processes. The potential for two advanced processes is also discussed in that report. The comparison of energy penalty and $\mathrm{CO}_{2}$ emission reduction is based on the full energy system, including mining, transportation, coal preparation, conversion, and gas treatment. Emissions associated with replacement power to compensate for the energy penalty of the $\mathrm{CO}_{2}$ recovery processes are included in the accounting. Compared with $\mathrm{CO}_{2}$ recovery from a conventional coal plant, the essential advantage of coupling a $\mathrm{CO}_{2}$ recovery system to a coal-gasification-based power plant is that removal of $\mathrm{CO}_{2}$ from gasifier fuel gas is more economical than removal of $\mathrm{CO}_{2}$ from flue gas produced by conventional coal combustion. Primarily, this economy results from the lesser dilution of the fuel gas with atmospheric nitrogen. Thus, a substantially smaller volume of gas must be processed, and the $\mathrm{CO}_{2}$ concentration in that gas is higher than in postcombustion flue gas. This advantage is expected to be more pronounced for a gasifier that uses oxygen rather than air as the oxidant. Further advantage is derived from the higher operating pressure associated with gasification in general and with the oxygen-blown case in particular.

Because of the dilution with nitrogen, air-blown gasifiers produce low-Btu gas, which has a heating value in the range of 90 to $170 \mathrm{Btu}$ per standard cubic foot (scf). Oxygen-blown gasifiers produce a medium-Btu gas, which has a heating value of about 250 to $400 \mathrm{Btu} / \mathrm{scf}$. In the air-blown case, substantially more of the energy value of the coal is manifested as sensible heat in the fuel gas. Losses associated with heat recovery and the cost of heat recovery equipment are therefore more important in the air-blown case. Thus, the economic value of hightemperature gas cleanup is greater in the air-blown case. The oxygen-blown cases considered here use low-temperature gas cleanup processes for sulfur removal. The air-blown cases considered in ANL/ESD-24 use a high-temperature system for sulfur removal.

\subsection{Goals, Objectives, and Approach}

The present volume supplements ANL/ESD-24. Four additional cases have been analyzed for this supplement. Table 1.1 summarizes the plant configurations for these cases. All four cases employ an oxygen-blown KRW gasifier with cold gas cleanup. Two cases use a gas turbine topping cycle and two cases use a fuel cell topping cycle. For the fuel cell cases, chilled methanol is used for $\mathrm{H}_{2} \mathrm{~S}$ recovery because of tight specifications $\left(\mathrm{H}_{2} \mathrm{~S}\right.$ at less than 1 part per million, volume [ppmv]) imposed to protect the fuel cell. For the gas turbine cases, a glycol- 
TABLE 1.1 Alternative Plant Configurations

\begin{tabular}{lllll}
\hline Case & $\mathrm{H}_{2}$ S Recovery & $\mathrm{CO}_{2}$ Recovery & Topping Cycle & Bottoming Cycle \\
\hline 1 & Glycol & Glycol & Gas turbine & Steam \\
2 & Glycol & Membrane & Gas turbine & Steam \\
3 & Chilled methanol & Glycol & Fuel cell & Steam \\
4 & Chilled methanol & Membrane & Fuel cell & Steam \\
\hline
\end{tabular}

based physical absorption system is used for $\mathrm{H}_{2} \mathrm{~S}$ recovery. These systems are analyzed for energy penalty and costs associated with the $\mathrm{CO}_{2}$ recovery system and for net $\mathrm{CO}_{2}$ removal. A comparison with the air-blown cases described in the earlier report is also provided. 


\section{Mining}

\subsection{Mining, Preparation, and Transportation of Raw Materials}

All seven cases presented here were adjusted to be on a consistent basis of 4,110 tons/d (stream day) of Illinois No. 6 coal from the Old Ben No. 26 mine. The underground mine is associated with a coal preparation plant. It is assumed that the IGCC power plant is $160 \mathrm{~km}$ from the mine and the coal is shipped by rail on a unit train. The ultimate analysis for this coal appears in Table 2.1. The impact on the energy budget of coal mining and shipment is $2.41 \mathrm{MW}$ of power use and $2,879 \mathrm{~kg} / \mathrm{h}$ of $\mathrm{CO}_{2}$ emissions.

Limestone is used for in-bed sulfur capture in the two air-blown gasifier cases. It is assumed that the limestone is extracted from a quarry about $160 \mathrm{~km}$ from the plant and transported by rail to the plant site. The impact on the energy budget of limestone mining and shipment is $0.27 \mathrm{MW}$ of power use and $406 \mathrm{~kg} / \mathrm{h}$ of $\mathrm{CO}_{2}$ emissions.

\subsection{Coal and Limestone Handling}

The coal preparation system for the $\mathrm{O}_{2}$-blown IGCC plant includes equipment for unloading the coal from the unit train, passing it through magnetic separators, and then conveying it to a hammermill. From there, the coal is conveyed to storage silos from which it is recovered in a fluidized stream for use in the gasifier. The coal is not dried for the $\mathrm{O}_{2}$-blown cases. The impact on the energy budget of coal preparation is $0.85 \mathrm{MW}$ of power use and no $\mathrm{CO}_{2}$ emissions (these will be combined with the overall emissions form the IGCC plant.) Drying the coal was not considered for this case.

By way of contrast, the coal preparation system for the air-blown IGCC plant includes equipment for unloading the coal from the unit train, passing it through magnetic separators, and then conveying it to silos for 14-h storage. The coal is crushed and dried in a series of three fluidized-bed roller mills. The heat for drying is provided by the hot $\left(760^{\circ} \mathrm{C}\right)$ flue gas from the IGCC sulfator process. This drying results in a significant amount of $\mathrm{CO}_{2}$ being emitted from the energy cycle that is not reclaimed and presents a possible opportunity for further reductions. The coal is then held in a 2-h bunker, from which it is pneumatically conveyed to surge bins ahead of the gasifier lockhoppers. The sulfator emits $11,374 \mathrm{~kg} / \mathrm{h}$ of $\mathrm{CO}_{2}$. Limestone is crushed in two pulverizers and then pneumatically conveyed to a 24-h storage silo and a 2-h storage bunker before being mixed with the coal in the gasifier surge bins. Energy consumption for coal and limestone preparation is $3.49 \mathrm{MW}$. 
TABLE 2.1 Analysis of Coal from Illinois No. 6 Seam, Old Ben No. 26 Mine

\begin{tabular}{|c|c|c|c|}
\hline Component & $\begin{array}{l}\text { Ultimate Analysis } \\
\text { as-Received } \\
\text { (wt \%) }\end{array}$ & Property & Value \\
\hline Moisture & 11.12 & Temperature of ash fusion (reducing conditions) $\left({ }^{\circ} \mathrm{C}\right)$ & \\
\hline Carbon & 63.75 & Initial deformation & 1,201 \\
\hline Hydrogen & 4.50 & Softening $(H=W)$ & 1,238 \\
\hline Nitrogen & 1.25 & Softening $(H=1 / 2 W)$ & 1,285 \\
\hline Chlorine & 0.29 & Fluid & 1,324 \\
\hline Sulfur & 2.51 & & \\
\hline Ash & 9.70 & Higher heating value $(\mathrm{J} / \mathrm{kg})$ & $27.13 \times 10^{6}$ \\
\hline Oxygen (by diff.) & 6.88 & & \\
\hline Total & 100.0 & & \\
\hline
\end{tabular}




\section{Oxygen-Blown Base Case with $\mathrm{No} \mathrm{CO}_{2}$ Recovery}

\subsection{Design Basis}

Figure 3.1 provides an overview of the base-case plant configuration, which does not incorporate $\mathrm{CO}_{2}$ recovery. This layout is typical of an oxygen-blown IGCC with cold-gas cleanup in which $\mathrm{H}_{2} \mathrm{~S}$ is removed by an acid gas removal system following gas cooling. The base-case analysis performed by Southern Company Services and others with sponsorship from the Electric Power Research Institute (EPRI 1990) assumes the use of Selexol ${ }^{\circledR}$, a commercial glycol-based process, for this $\mathrm{H}_{2} \mathrm{~S}$ removal. The cleaned gas is then saturated and reheated with steam before it is used in the gas turbine. The turbine exhaust gas is used to raise steam for a Rankine cycle steam plant. Steam from the heat recovery steam generator is also supplied to the gasifier. Oxidant is provided by an air separation plant. Three KRW gasifiers with the capacity to provide $42 \%$ of plant requirements are used to ensure high reliability.

The oxygen is produced by cryogenic distillation in a separate air plant that is not integrated with the gasifier and power generation systems except through direct use of the oxygen product. Opportunities for integration do exist but are not incorporated in current plans for oxygen-blown gasifiers. The KRW gasifier is an agglomerating fluidized-bed reactor that operates at $450 \mathrm{lb} / \mathrm{in} .^{2}$ gauge (psig) and $1,850^{\circ} \mathrm{F}$. Operation in the agglomerating regime enhances overall plant performance (EPRI 1990; Takematsu 1991). The KRW process has been demonstrated in extensive pilot scale tests, but no commercial demonstration unit has been built. One commercial-scale air-blown unit is under construction.

Hot gas from the gasification reactor contains ash, char, and sulfur species that must be removed before combustion. Ninety-five percent of the ash and char are removed in cyclones after the initial cooling of the hot $\left(1,850^{\circ} \mathrm{F}\right)$ raw gas to $1,350^{\circ} \mathrm{F}$. Following further cooling to $450^{\circ} \mathrm{F}$, the remaining fines are removed by sintered metal filters. Final cooling to $100^{\circ} \mathrm{F}$ is accomplished by water quench prior to acid gas removal by the Selexol process. The concentrated $\mathrm{H}_{2} \mathrm{~S}$ stream from the Selexol process is treated in a Claus unit for sulfur recovery. Design sulfur recovery is $96.4 \%$.

\subsection{Material Balance}

Material flows are summarized in Table 3.1,1 which provides a comparison of the reference oxygen-blown base case with an air-blown base case using in-bed sulfur capture.

\footnotetext{
1 Design specifications used in this report are a combination of specifications from two documents. Assessment of Coal Gasification/Hot Gas Cleamup Based on Advanced Gas Turbine Systems (Gallaspy 1990b) provided the design basis for the air-blown systems reviewed in ANL/ESD-24. This document also includes limited information on one oxygen-blown case, an update of a design evaluated in an earlier report, Southern Company Service's Study of a KRW-Based GCC Power Plant (Gallaspy 1990a). This earlier report has been relied on for certain design details, although flows have been scaled to agree with the updated plant specifications in the former report. The update is primarily a result of a substantial increase in the performance rating of the GE gas turbine selected as part of the design basis.
} 


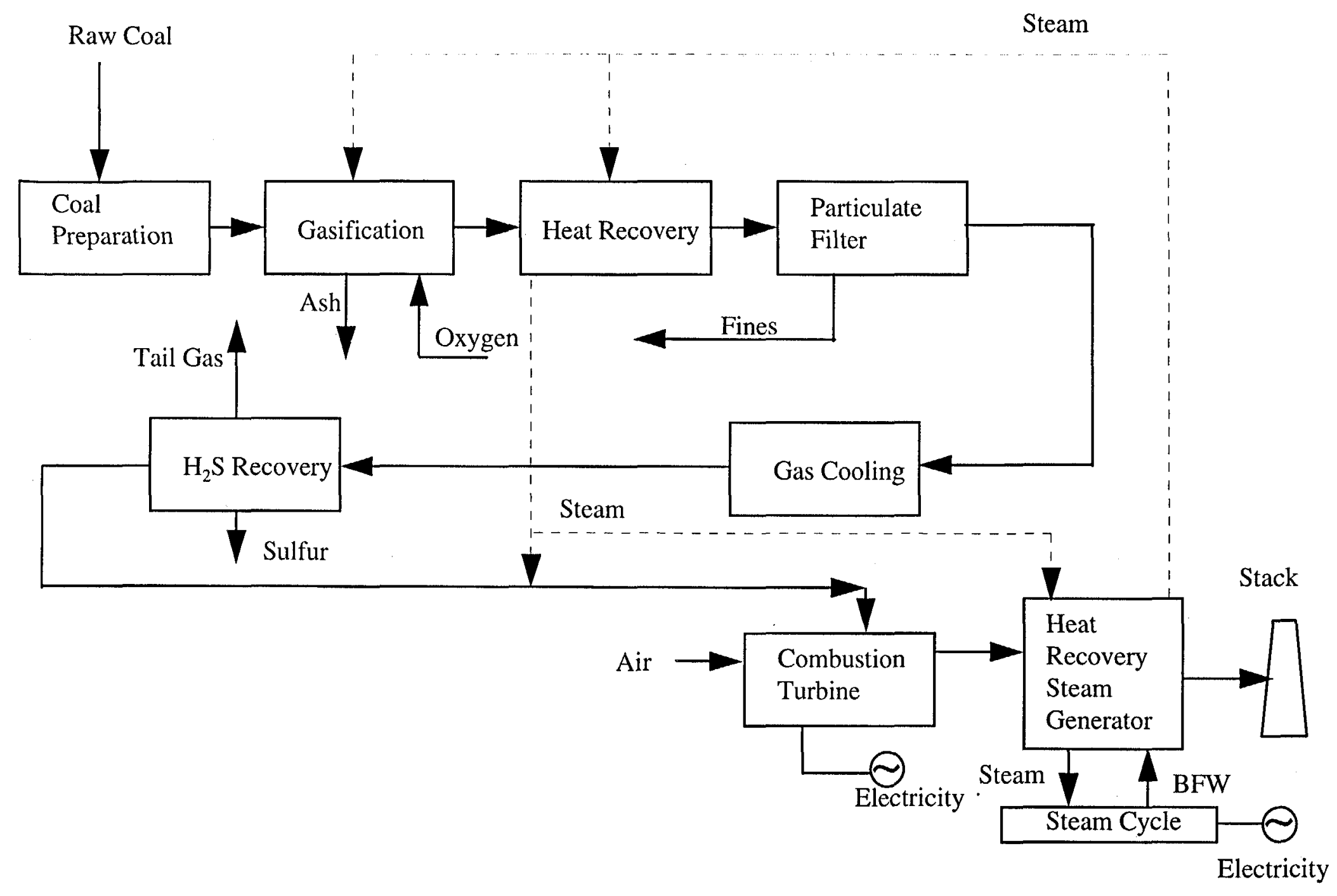

FIGURE 3.1 Block Diagram of the Base-Case Oxygen-Blown KRW IGCC System 
TABLE 3.1 Material Flows for Oxygen-Blown and Air-Blown Base Cases

\begin{tabular}{|c|c|c|}
\hline Material Flow (tons/d) & Oxygen-Blown Base Case & Air-Blown Base Case \\
\hline Coal (prepared) & 3,845 & 3,792 \\
\hline Limestone & 0 & 1,053 \\
\hline Air & 0 & 12,888 \\
\hline Oxygen & 2,347 & 0 \\
\hline Solid waste & 492 & 1,231 \\
\hline Sulfur & 78 & 0 \\
\hline $\mathrm{CO}_{2}$ (gasifier only) & 8,586 & 9,600 \\
\hline $\mathrm{SO}_{2}$ (gasifier only) & 6.92 & 1.24 \\
\hline Net power output (MW) & 413.5 & 458.4 \\
\hline
\end{tabular}

\subsection{Gas Turbine, Steam Cycle, and Plant Performance}

Nominal capacity of the reference plant is $413.5 \mathrm{MW}$ net, including $298.8 \mathrm{MW}$ from the gas turbines and 159.4 MW from the steam cycle minus $44.7 \mathrm{MW}$ for station service load. The net plant heat rate is $9,039 \mathrm{Btu} / \mathrm{kWh}$ at full load. The power island incorporates two GE MS7001F combustion turbines, two heat recovery steam generators, and one reheat steam turbine.

\subsection{Economics}

A summary of capital and operating costs is provided in Section 9. 


\section{Case 1 - Gas Turbine Topping Cycle and Glycol $\mathrm{CO}_{2}$ Recovery}

As noted in the introduction, two topping cycle options have been studied: gas turbines and fuel cells. Two $\mathrm{CO}_{2}$-recovery options have been investigated for use in conjunction with the gas turbine topping cycle: a glycol-based absorption system and a two-stage membrane system. Detailed design, performance, and cost information is presented in this section for the gas turbine option with glycol-based $\mathrm{CO}_{2}$ recovery. A glycol system is also used for sulfur recovery.

\subsection{Design Basis}

Figure 4.1 shows the addition of a glycol-based $\mathrm{CO}_{2}$ recovery system to the reference IGCC plant. The membrane system occupies a similar position in the overall scheme, although stream conditions differ somewhat for the two recovery options. The $\mathrm{CO}_{2}$ recovery follows $\mathrm{H}_{2} \mathrm{~S}$ recovery, which is preceded by a shift reaction to convert the CO-rich synthesis gas to a hydrogen-rich gas diluted by $\mathrm{CO}_{2}$. This shift is accomplished in two stages for economical use of catalysts and is integrated with the power cycle by heat exchange with the $\mathrm{CO}_{2}$-lean fuel gas. The role of these processes is clarified in Figure 4.2, which displays the gas composition at various process stages. Note the dramatic increase in $\mathrm{CO}_{2}$ during the shift reaction and the simultaneous reduction in $\mathrm{CO}$. The removal of $\mathrm{CO}_{2}$ is evident by contrast of the absorber inlet concentration and the dry fuel gas product. Nominally $90 \% \mathrm{CO}_{2}$ recovery is accomplished by a combination of $95 \%$ conversion of $\mathrm{CO}$ in the shift and $95 \%$ recovery of the resulting $\mathrm{CO}_{2}$ in the gylcol process. Somewhat less recovery is accomplished in the membrane case because of membrane performance limitations. Table 4.1 is a summary of principal material flows for the base case and for this design option.

\subsection{Shift Reactor}

The shift reactor relies on steam in the presence of a catalyst to convert $\mathrm{CO}$ to $\mathrm{CO}_{2}$. Catalyst performance is temperature sensitive, so that reduction in gas stream temperature is required for efficient conversion. Economic use of catalysts dictates that the shift reaction be carried out in two stages. In the first stage, an iron-based catalyst is used, which is effective above $650^{\circ} \mathrm{F}$. In the second stage, a copper-based catalyst is used, which is effective at lower temperatures. Cooling is required before both stages to remove sensible heat and heat of reaction associated with the shift reaction. The effective use of the heat removed in cooling the gas is an important design consideration. The shift system design is discussed in detail in ANL/ESD-24. In that report, it is demonstrated that a considerable overall cycle efficiency advantage is gained by allocating as much of the sensible heat as possible to the cleaned fuel gas feed to the turbine. A similar design is incorporated here. This involves the optimization of the two catalytic reactors and of the heat integration. Figure 4.3 is a flow diagram of the shift reactor system showing the heat integration. The high-temperature heating and humidification of the fuel gas stream is 


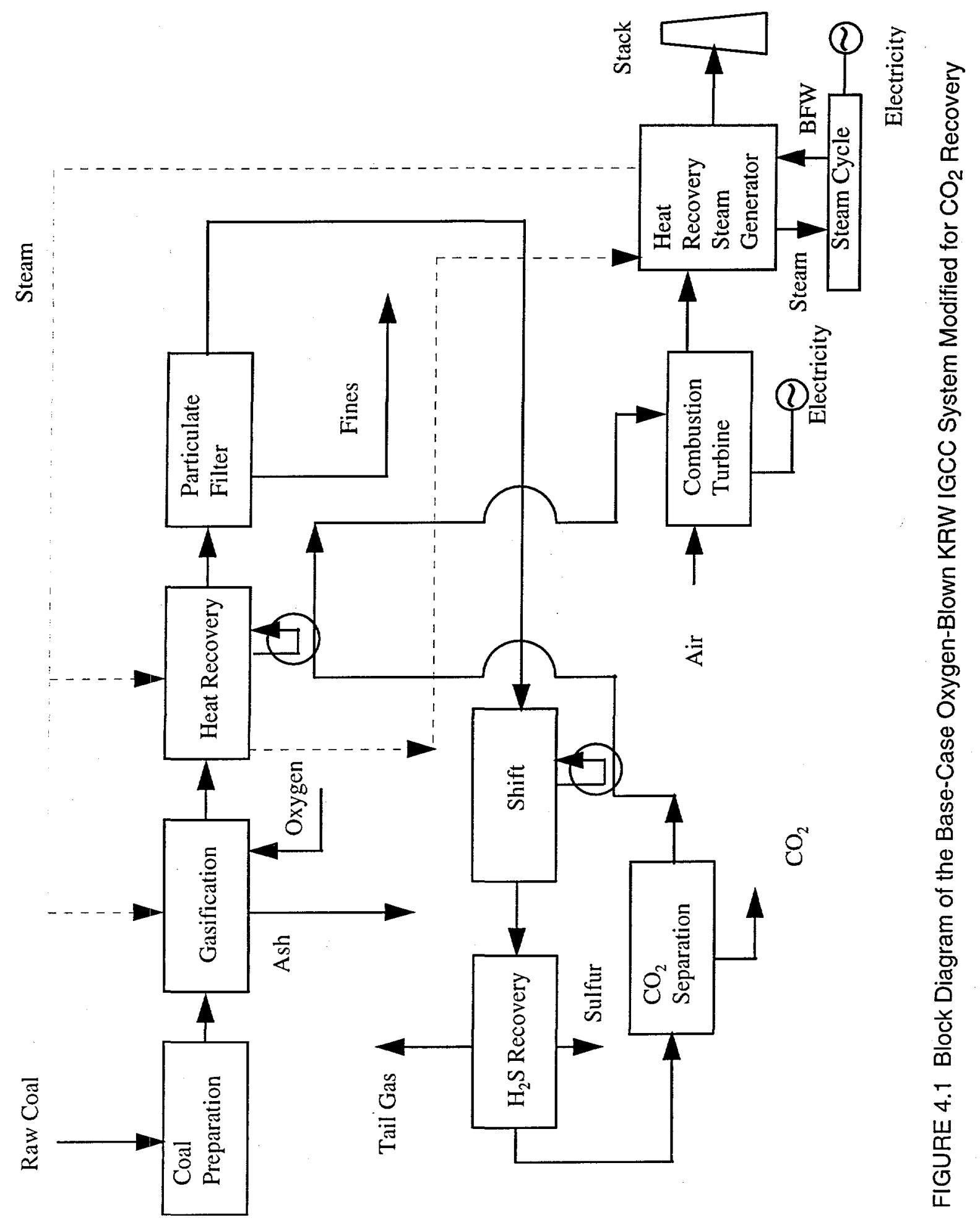




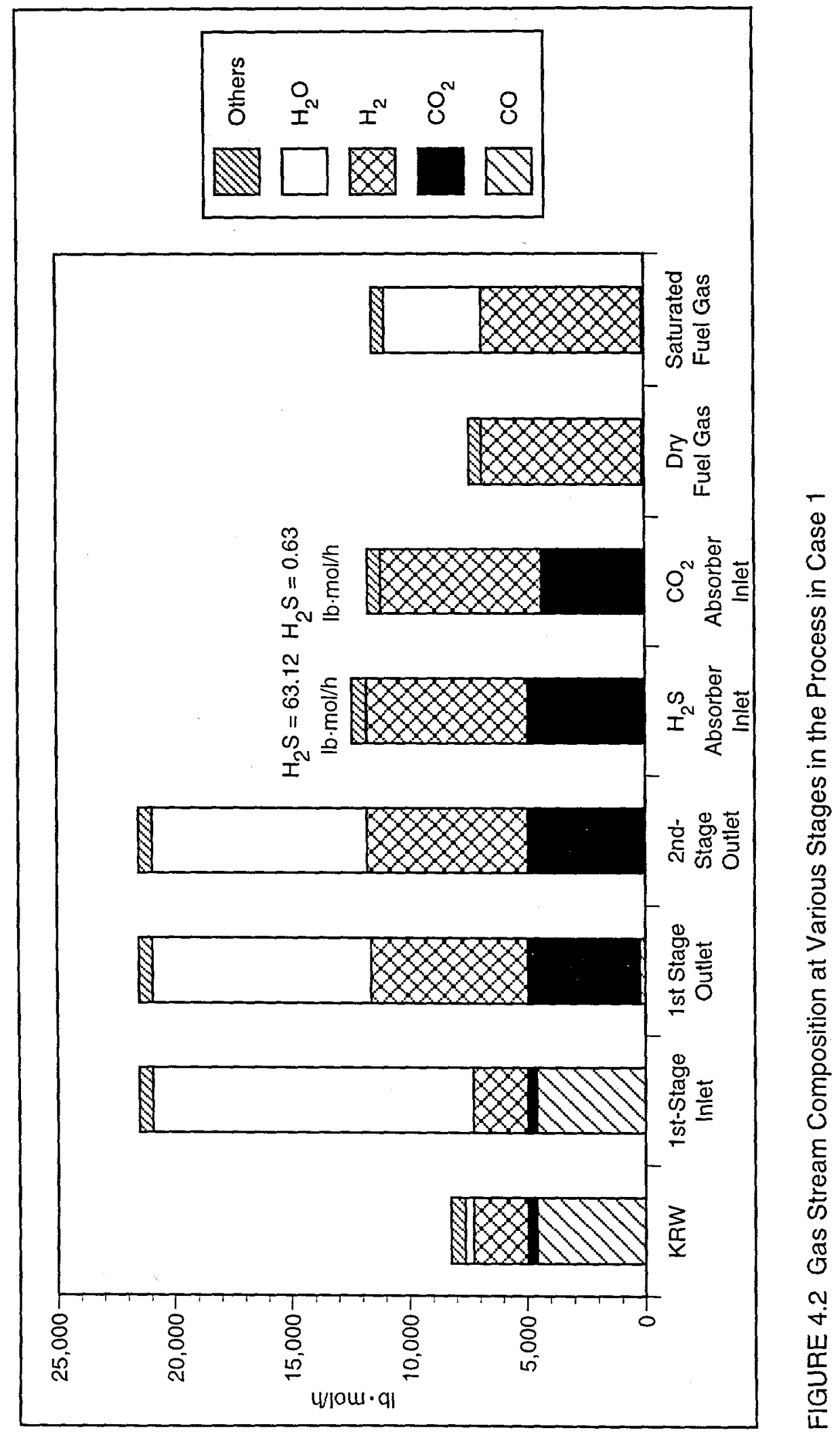


TABLE 4.1 Material Flows for Oxygen-Blown Base Case and Case 1

\begin{tabular}{|c|c|c|}
\hline Material Flow (tons/d) & Base Case & Case 1 \\
\hline Coal (prepared) & 3,845 & 3,845 \\
\hline Limestone & 0 & 0 \\
\hline Air & 0 & 0 \\
\hline Oxygen & 2,347 & 2,347 \\
\hline Solid waste & 492 & 492 \\
\hline Sulfur & 78 & 78 \\
\hline $\mathrm{CO}_{2}$ (gasifier only) & 8,586 & 898 \\
\hline $\mathrm{SO}_{2}$ (gasifier only) & 6.92 & 6.92 \\
\hline Net power output (MW) & 413.5 & 377.47 \\
\hline
\end{tabular}

accomplished with the initial cooling of the synthesis gas. The allocation of available enthalpy is summarized in Table 4.2. Details on the gas stream composition and the other streams shown in Figure 4.3 are provided in Table 4.3 .

\subsection{Glycol Process for $\mathrm{CO}_{2}$ and $\mathrm{H}_{2} \mathrm{~S}$ Recovery}

Of the several commercial options for $\mathrm{CO}_{2}$ recovery investigated in ANL/ESD-24, the glycol process had the most favorable economics and the lowest energy penalty. The design analyzed here is based on a commercial version of the glycol process; it is called Selexol ${ }^{\circledR}$. Lack of design data for this proprietary process makes system optimization to commercial standards impossible, but the key features of a commercial system are well-represented by this analysis. A glycol process has also been employed for $\mathrm{H}_{2} \mathrm{~S}$ recovery in the two gas turbine cases. Figure 4.4 is a flow diagram of the glycol process for $\mathrm{H}_{2} \mathrm{~S}$ removal. The material balances for the flows represented in that figure are summarized in Table 4.4. Key assumptions for these stream flow calculations are presented in Table 4.5. A similar set of exhibits defines the glycol system for $\mathrm{CO}_{2}$ recovery. A significant difference between the two systems is the use of thermal stripping for solvent recovery in the $\mathrm{H}_{2} \mathrm{~S}$ case and flash recovery in the $\mathrm{CO}_{2}$ case. Figure 4.5 shows the glycol recovery process for the $\mathrm{CO}_{2}$. The stream flow data and stream calculation descriptions are summarized in Tables 4.6 and 4.7 , respectively.

\subsection{Gas Turbine, Steam Cycle, and Plant Performance}

The application of $\mathrm{CO}_{2}$ recovery by the glycol process results in a reduction in net plant output of $36 \mathrm{MW}$ or $8.7 \%$ of the reference case plant output. Table 4.8 lists the gas turbine output, steam cycle output, and internal plant consumption for the base case (no $\mathrm{CO}_{2}$ recovery) and for the glycol-based $\mathrm{CO}_{2}$ recovery case. The most significant losses are a reduction in gas turbine output and the consumption of power for $\mathrm{CO}_{2}$ compression. 


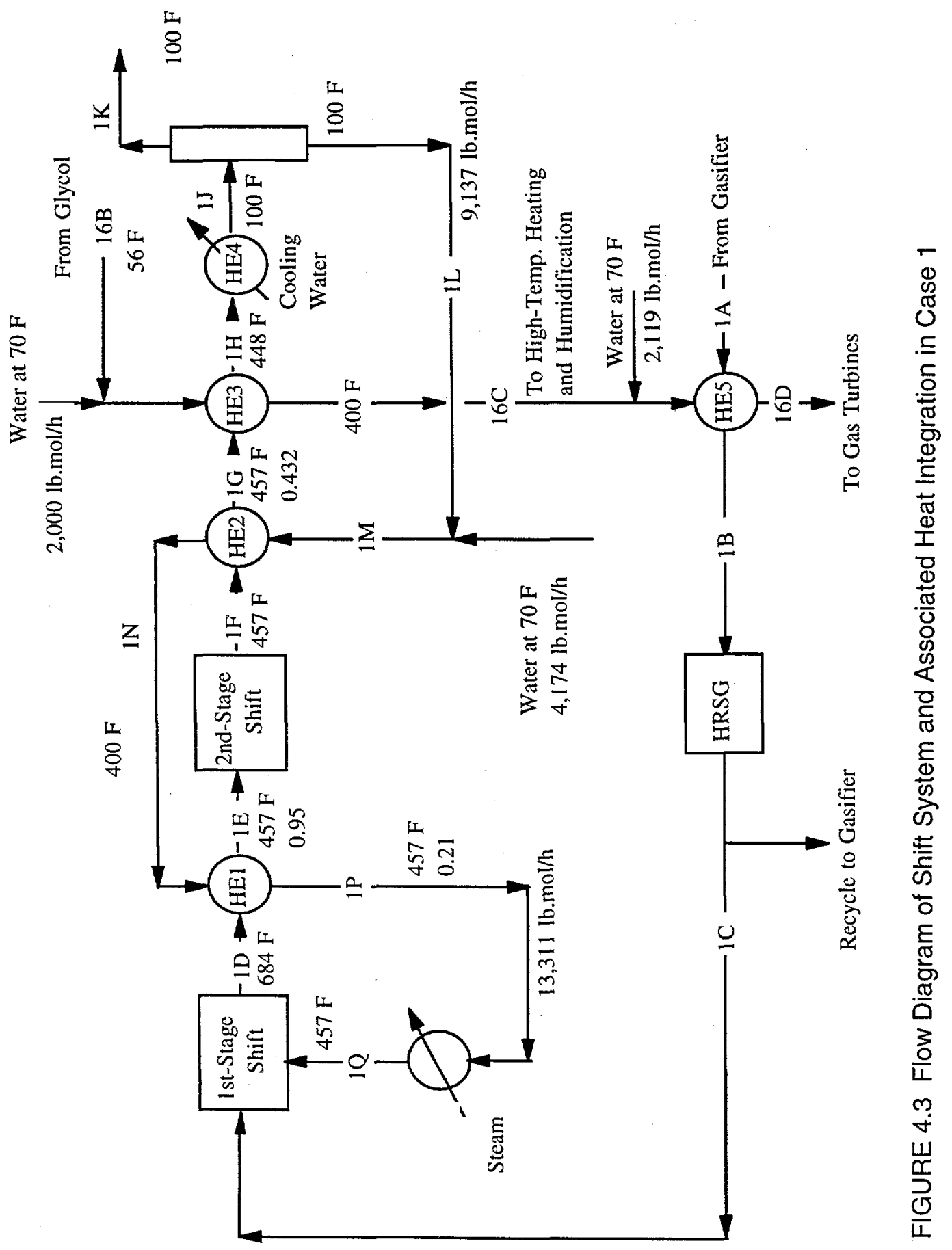


TABLE 4.2 Heat Recovery and Allocation ( $\left.10^{6} \mathrm{Btu} / \mathrm{h}\right)$ for Gas Turbine/Glycol Process in Case 1

\begin{tabular}{lcccc}
\hline \multicolumn{1}{c}{ Process } & $\begin{array}{c}\text { Enthalpy Change } \\
\text { Available from } \\
\text { Process }\end{array}$ & $\begin{array}{c}\text { Allocation to } \\
\text { Fuel Gas } \\
\text { Preheating }\end{array}$ & $\begin{array}{c}\text { Allocation for } \\
\text { Raising Steam for } \\
\text { Shift System }\end{array}$ & $\begin{array}{c}\text { Allocation } \\
\text { to Steam } \\
\text { Cycle }\end{array}$ \\
\hline Initial gas cooling to $460^{\circ} \mathrm{F}$ & 513.89 & 344.28 & 123.89 & 45.71 \\
Cooling after first-stage shift & 168.21 & 0.00 & 168.21 & 0.00 \\
Cooling after second-stage shift & 673.27 & 177.41 & 215.65 & 280.22 \\
\hline
\end{tabular}


TABLE 4.3 Stream Flows of Shift System of Gas Turbine/Glycol Process in Case 1

\begin{tabular}{|c|c|c|c|c|c|c|}
\hline Stream Data & Stream $1 \mathrm{~A}$ & Stream $1 B$ & Stream $1 \mathrm{C}$ & Stream 1D & Stream $1 E$ & Stream $1 \mathrm{~F}$ \\
\hline Description of stream & $\begin{array}{l}\text { Raw gas from KRW } \\
\text { gasifier }\end{array}$ & $\begin{array}{l}\text { Raw gases after } \\
\text { gas-gas heat } \\
\text { exchanger }\end{array}$ & $\begin{array}{l}\text { Raw gases to shift } \\
\text { system }\end{array}$ & $\begin{array}{l}\text { Raw gas from 1st- } \\
\text { stage shift }\end{array}$ & $\begin{array}{l}\text { Raw gases from } \\
\text { heat exchanger } 1\end{array}$ & $\begin{array}{l}\text { Raw gas trom } \\
\text { 2nd-stage shift }\end{array}$ \\
\hline \multicolumn{7}{|l|}{ Gases (lb.mol/h) } \\
\hline $\mathrm{CO}$ & $8,887.28$ & $8,887.28$ & $4,558.89$ & 227.94 & 227.94 & 45.59 \\
\hline $\mathrm{CO}_{2}$ & 769.57 & 769.57 & 394.76 & $4,725.71$ & $4,725.71$ & $4,908.06$ \\
\hline $\mathrm{H}_{2}$ & $4,513.52$ & $4,513.52$ & $2,315.43$ & $6,646.37$ & $6,646.37$ & $6,828.72$ \\
\hline $\mathrm{H}_{2} \mathrm{O}$ & 711.96 & 711.96 & 365.22 & $9,345.71$ & $9,345.71$ & $9,163.36$ \\
\hline $\mathrm{N}_{2}$ & 71.03 & 71.03 & 36.43 & 36.43 & 36.43 & 36.43 \\
\hline $\operatorname{Ar}$ & 141.76 & 141.76 & 72.72 & 72.72 & 72.72 & 72.72 \\
\hline $\mathrm{CH}_{4}$ & 950.15 & 950.15 & 487.39 & 487.39 & 487.39 & 487.39 \\
\hline $\mathrm{NH}_{3}$ & 36.61 & 36.61 & 18.79 & 18.79 & 18.79 & 18.79 \\
\hline $\mathrm{H}_{2} \mathrm{~S}$ & 123.05 & 123.05 & 63.12 & 63.12 & 63.12 & 63.12 \\
\hline $\mathrm{HCN}$ & 0.80 & 0.80 & 0.41 & 0.41 & 0.41 & 0.41 \\
\hline $\mathrm{O}_{2}$ & 0.00 & 0.00 & 0.00 & 0.00 & 0.00 & 0.00 \\
\hline $\cos$ & 14.49 & 14.49 & 7.43 & 7.43 & 7.43 & 7.43 \\
\hline $\mathrm{SO}_{2}$ & 0.00 & 0.00 & 0.00 & 0.00 & 0.00 & 0.00 \\
\hline Total gas flow & $16,220.23$ & $16,220.23$ & $8,320.59$ & $21,632.03$ & $21,632.03$ & $21,632.03$ \\
\hline \multicolumn{7}{|l|}{ Liquids $(\mathrm{lb} \cdot \mathrm{mol} / \mathrm{h})$} \\
\hline $\mathrm{H}_{2} \mathrm{O}$ & 0.00 & 0.00 & 0.00 & 0.00 & 0.00 & 0.00 \\
\hline Temperature $\left({ }^{\circ} \mathrm{F}\right)$ & $1,749.45$ & 934.83 & 457.40 & 683.87 & 457.40 & 457.40 \\
\hline Pressure (psia) & 465.00 & 465.00 & 457.00 & 457.00 & 457.00 & 457.00 \\
\hline $\begin{array}{l}\text { Enthalpy of stream }(B t u / h) \\
\left.\text { (reference, } 32^{\circ} \mathrm{F}\right)\end{array}$ & $240,514,724$ & $125,753,677$ & $32,874,692$ & $295,099,586$ & $239,029,336$ & $242,258,675$ \\
\hline
\end{tabular}


TABLE 4.3 (Cont.)

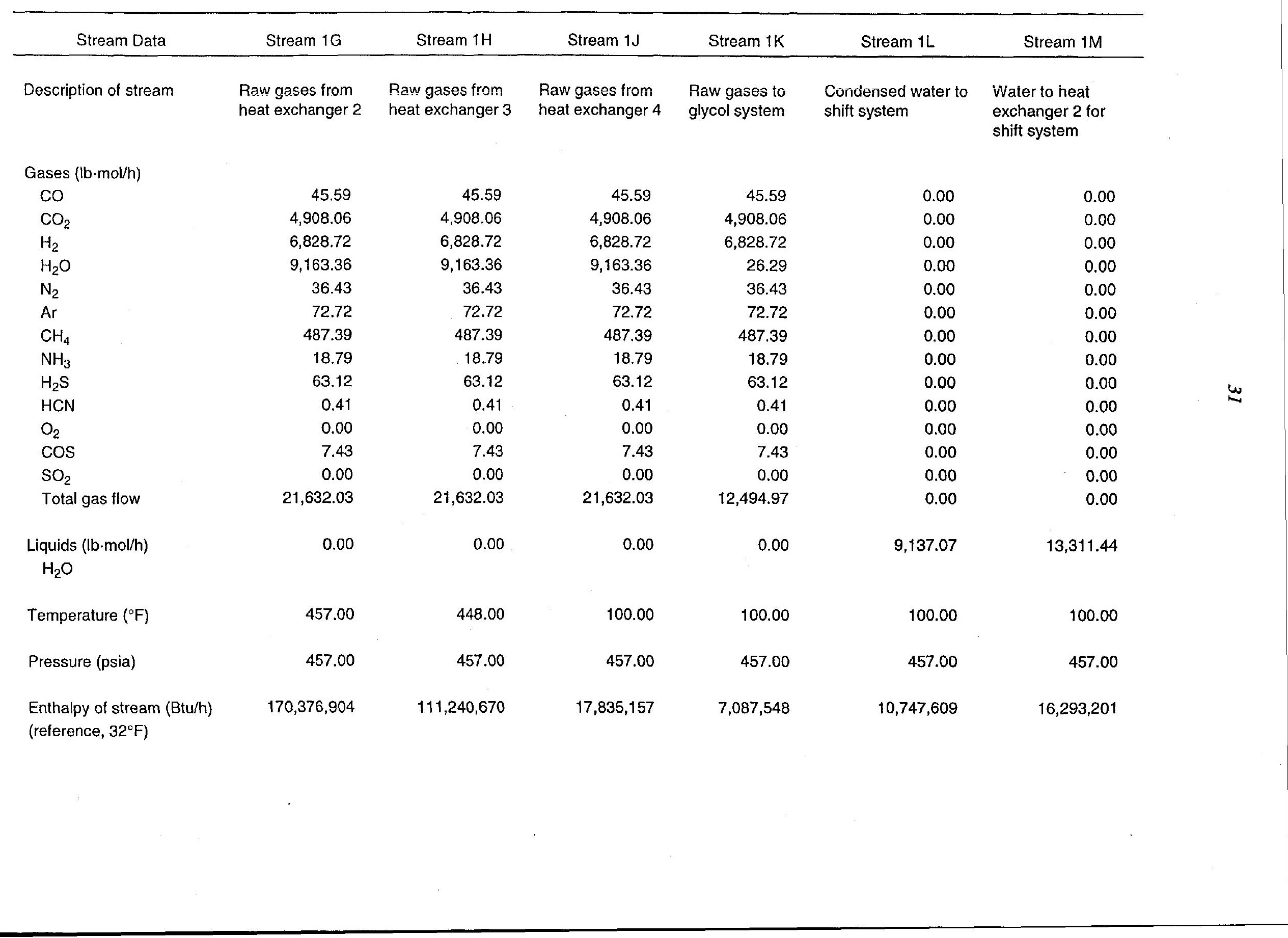


TABLE 4.3 (Cont.)

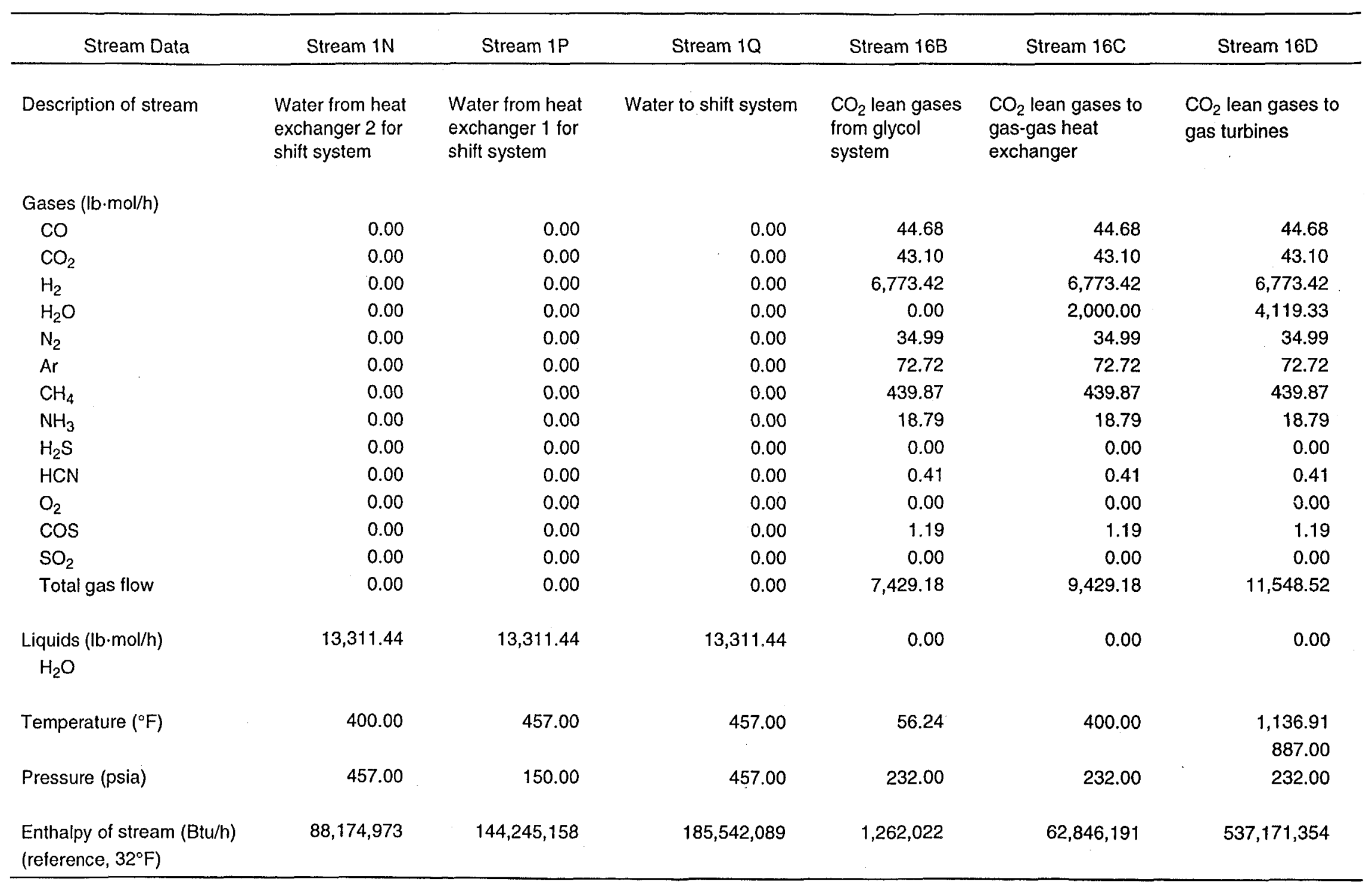




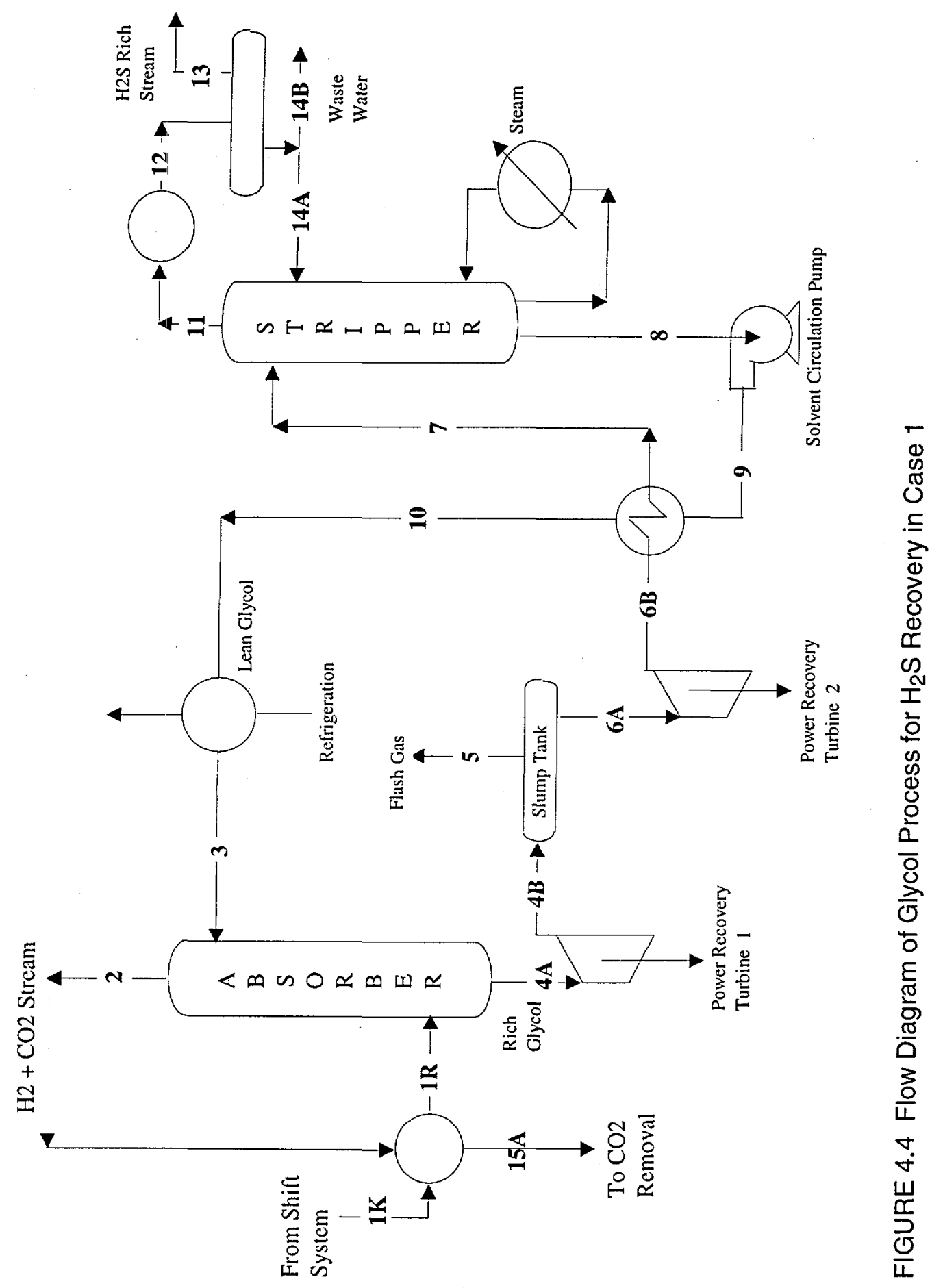


TABLE 4.4 Stream Flows of Glycol Process for $\mathrm{H}_{2} \mathrm{~S}$ Removal in Case 1

\begin{tabular}{|c|c|c|c|c|c|c|}
\hline Stream Data & Stream $1 \mathrm{~K}$ & Stream 1R & Stream 2 & Stream 3 & Stream $4 A$ & Stream 4B \\
\hline Description of stream & $\begin{array}{l}\text { Feed gas from } \\
\text { shift system }\end{array}$ & Absorber feed & $\begin{array}{l}\text { Sulfur-free gas } \\
\text { from absorber }\end{array}$ & Lean glycol solvent & $\begin{array}{l}\text { Rich glycol solvent } \\
\text { from absorber }\end{array}$ & $\begin{array}{l}\text { Rich glycol solvent } \\
\text { after turbine } 1\end{array}$ \\
\hline \multicolumn{7}{|l|}{ Gases $(\mathrm{lb} \cdot \mathrm{mol} / \mathrm{h})$} \\
\hline $\mathrm{co}$ & 45.59 & 45.59 & 45.13 & 0.00 & 0.46 & 0.46 \\
\hline $\mathrm{CO}_{2}$ & $4,908.06$ & $4,908.06$ & $4,310.02$ & 109.53 & 707.57 & 707.57 \\
\hline $\mathrm{H}_{2}$ & $6,828.72$ & $6,828.72$ & $6,822.47$ & 10.95 & 17.20 & 17.20 \\
\hline $\mathrm{H}_{2} \mathrm{O}$ & 26.29 & 26.29 & 0.00 & $1,022.29$ & $1,048.58$ & $1,048.58$ \\
\hline $\mathrm{N}_{2}$ & 36.43 & 36.43 & 35.71 & 0.00 & 0.73 & 0.73 \\
\hline $\operatorname{Ar}$ & 72.72 & 72.72 & 72.72 & 0.00 & 0.00 & 0.00 \\
\hline $\mathrm{CH}_{4}$ & 487.39 & 487.39 & 463.02 & 0.00 & 24.37 & 24.37 \\
\hline $\mathrm{NH}_{3}$ & 18.79 & 18.79 & 18.79 & 0.00 & 0.00 & 0.00 \\
\hline$\dot{H}_{2} \mathrm{~S}$ & 63.12 & 63.12 & 0.63 & 21.91 & 84.40 & 84.40 \\
\hline $\mathrm{HCl}$ & 0.41 & 0.41 & 0.41 & 0.00 & 0.00 & 0.00 \\
\hline $\mathrm{O}_{2}$ & 0.00 & 0.00 & 0.00 & 0.00 & 0.00 & 0.00 \\
\hline $\cos$ & 7.43 & 7.43 & 2.97 & 0.00 & 4.46 & 4.46 \\
\hline $\mathrm{SO}_{2}$ & 0.00 & 0.00 & 0.00 & 0.00 & 0.00 & 0.00 \\
\hline Total gas flow & $12,494.97$ & $12,494.97$ & $11,771.88$ & $1,164.68$ & $1,887.76$ & $1,887.76$ \\
\hline \multicolumn{7}{|l|}{ Liquids $(\mathrm{lb} \cdot \mathrm{mol} / \mathrm{h})$} \\
\hline Glycol solvent & 0.00 & 0.00 & 0.00 & $2,190.62$ & $2,190.62$ & $2,190.62$ \\
\hline Temperature $\left({ }^{\circ} \mathrm{F}\right)$ & 100.00 & 63.00 & 30.00 & 30.00 & 63.62 & 62.28 \\
\hline Pressure (psia) & 451.00 & 451.00 & 446.00 & 451.00 & 446.00 & 100.00 \\
\hline $\begin{array}{l}\text { Enthalpy }(\mathrm{Btu} / \mathrm{h}) \\
\left.\text { (reference, } 32^{\circ} \mathrm{F}\right)\end{array}$ & $7,087,548$ & $3,456,963$ & $-180,609$ & $-640,350$ & $10,332,246$ & $9,893,895$ \\
\hline
\end{tabular}


TABLE 4.4 (Cont.)

\begin{tabular}{|c|c|c|c|c|c|c|}
\hline Stream Data & Stream 5 & Stream $6 A$ & Stream 6B & Stream 7 & Stream 8 & Stream 9 \\
\hline Description of stream & Flash gas & $\begin{array}{l}\text { Rich glycol solvent } \\
\text { to turbine } 2\end{array}$ & $\begin{array}{l}\text { Rich glycol solvent } \\
\text { from turbine } 2\end{array}$ & $\begin{array}{l}\text { Rich glycol solvent } \\
\text { after heat exchange }\end{array}$ & $\begin{array}{l}\text { Lean giycoi solvent } \\
\text { from stripper }\end{array}$ & $\begin{array}{l}\text { Lean glycol solvent } \\
\text { after circulation pump }\end{array}$ \\
\hline \multicolumn{7}{|l|}{ Gases $(\mathrm{lb} \cdot \mathrm{mol} / \mathrm{h})$} \\
\hline $\mathrm{CO}$ & 0.41 & 0.05 & 0.05 & 0.05 & 0.00 & 0.00 \\
\hline $\mathrm{CO}_{2}$ & 566.06 & 141.51 & 141.51 & 141.51 & 109.53 & 109.53 \\
\hline $\mathrm{H}_{2}$ & 6.19 & 11.01 & 11.01 & 11.01 & 10.95 & 10.95 \\
\hline $\mathrm{H}_{2} \mathrm{O}$ & 10.49 & $1,038.10$ & $1,038.10$ & $1,038.10$ & $1,022.29$ & $1,022.29$ \\
\hline $\mathrm{N}_{2}$ & 0.66 & 0.07 & 0.07 & 0.07 & 0.00 & 0.00 \\
\hline $\mathrm{Ar}$ & 0.00 & 0.00 & 0.00 & 0.00 & 0.00 & 0.00 \\
\hline $\mathrm{CH}_{4}$ & 20.71 & 3.66 & 3.66 & 3.66 & 0.00 & 0.00 \\
\hline $\mathrm{NH}_{3}$ & 0.00 & 0.00 & 0.00 & 0.00 & 0.00 & 0.00 \\
\hline $\mathrm{H}_{2} \mathrm{~S}$ & 4.22 & 80.18 & 80.18 & 80.18 & 21.91 & 21.91 \\
\hline $\mathrm{HCl}$ & 0.00 & 0.00 & 0.00 & 0.00 & 0.00 & 0.00 \\
\hline $\mathrm{O}_{2}$ & 0.00 & 0.00 & 0.00 & 0.00 & 0.00 & 0.00 \\
\hline $\cos$ & 2.23 & 2.23 & 2.23 & 2.23 & 0.00 & 0.00 \\
\hline $\mathrm{SO}_{2}$ & 0.00 & 0.00 & 0.00 & 0.00 & 0.00 & 0.00 \\
\hline Total gas flow & 610.96 & $1,276.80$ & $1,276.80$ & $1,276.80$ & $1,164.68$ & $1,164.68$ \\
\hline \multicolumn{7}{|l|}{ Liquids $(\mathrm{lb} \cdot \mathrm{mol} / \mathrm{h})$} \\
\hline Glycol solvent & 0.00 & $2,190.62$ & $2,190.62$ & $2,190.62$ & $2,190.62$ & $2,190.62$ \\
\hline Temperature $\left({ }^{\circ} \mathrm{F}\right)$ & 42.44 & 42.44 & 42.10 & 190.00 & 212.00 & 215.21 \\
\hline Pressure (psia) & 100.00 & 100.00 & 14.70 & 14.70 & 14.70 & 451.00 \\
\hline $\begin{array}{l}\text { Enthalpy }(B t u / h) \\
\left.\text { (reference, } 32^{\circ} \mathrm{F}\right)\end{array}$ & 589.41 & $3,354,029$ & $3,245,651$ & $50,770,201$ & $57,640,407$ & $58,667,747$ \\
\hline
\end{tabular}


TABLE 4.4 (Cont.)

\begin{tabular}{|c|c|c|c|c|c|c|c|}
\hline Stream Data & Stream 10 & Stream 11 & Stream 12 & Stream 13 & Stream 14A & Stream 14B & Stream 15A \\
\hline Description of stream & $\begin{array}{l}\text { Lean glycol solvent } \\
\text { after heat exchange }\end{array}$ & $\begin{array}{l}\mathrm{H}_{2} \mathrm{~S} \text {-rich gas } \\
\text { from stripper }\end{array}$ & $\begin{array}{l}\mathrm{H}_{2} \mathrm{~S} \text {-rich } \\
\text { gas after } \\
\text { condenser }\end{array}$ & $\mathrm{H}_{2} \mathrm{~S}$-rich gas & $\begin{array}{l}\text { Recycle to } \\
\text { stripper }\end{array}$ & $\begin{array}{l}\text { Wastewater to } \\
\text { dișposal }\end{array}$ & $\begin{array}{l}\text { Sulfur-free fuel gas } \\
\text { after heat exchange }\end{array}$ \\
\hline \multicolumn{8}{|l|}{ Gases $(\mathrm{lb} \cdot \mathrm{mol} / \mathrm{h})$} \\
\hline $\mathrm{CO}$ & 0.00 & 0.05 & 0.05 & 0.05 & 0.00 & 0.00 & 45.13 \\
\hline $\mathrm{CO}_{2}$ & 109.53 & 31.98 & 31.98 & 31.98 & 0.00 & 0.00 & $4,310.02$ \\
\hline $\mathrm{H}_{2}$ & 10.95 & 0.06 & 0.06 & 0.06 & 0.00 & 0.00 & $6,822.47$ \\
\hline $\mathrm{H}_{2} \mathrm{O}$ & $1,022.29$ & $1,038.10$ & $1,038.10$ & 4.92 & $1,022.29$ & 10.88 & 0.00 \\
\hline $\mathrm{N}_{2}$ & 0.00 & 0.07 & 0.07 & 0.07 & 0.00 & 0.00 & 35.71 \\
\hline $\mathrm{Ar}$ & 0.00 & 0.00 & 0.00 & 0.00 & 0.00 & 0.00 & 72.72 \\
\hline $\mathrm{CH}_{4}$ & 0.00 & 3.66 & 3.66 & 3.66 & 0.00 & 0.00 & 463.02 \\
\hline $\mathrm{NH}_{3}$ & 0.00 & 0.00 & 0.00 & 0.00 & 0.00 & 0.00 & 18.79 \\
\hline $\mathrm{H}_{2} \mathrm{~S}$ & 21.91 & 58.27 & 58.27 & 58.27 & 0.00 & 0.00 & 0.63 \\
\hline $\mathrm{HCl}$ & 0.00 & 0.00 & 0.00 & 0.00 & 0.00 & 0.00 & 0.41 \\
\hline $\mathrm{O}_{2}$ & 0.00 & 0.00 & 0.00 & 0.00 & 0.00 & 0.00 & 0.00 \\
\hline $\cos$ & 0.00 & 2.23 & 2.23 & 2.23 & 0.00 & 0.00 & 2.97 \\
\hline $\mathrm{SO}_{2}$ & 0.00 & 0.00 & 0.00 & 0.00 & 0.00 & 0.00 & 0.00 \\
\hline Total gas flow & $1,164.68$ & $1,134.41$ & $1,134.41$ & 101.24 & $1,022.29$ & 10.88 & $11,771.88$ \\
\hline Liquids $(l b \cdot \mathrm{mol} / \mathrm{h})$ & & & & & . & & \\
\hline Glycol solvent & $2,190.62$ & 0.00 & 0.00 & 0.00 & 0.00 & 0.00 & 0.00 \\
\hline Temperature $\left({ }^{\circ} \mathrm{F}\right)$ & 66.80 & 212.00 & 100.00 & 100.00 & 100.00 & 100.00 & 70.00 \\
\hline Pressure (psia) & 14.70 & 14.70 & 14.70 & 14.70 & 14.70 & 14.70 & 446.00 \\
\hline $\begin{array}{l}\text { Enthalpy }(\mathrm{Btu} / \mathrm{h}) \\
\text { (reference, } 32^{\circ} \mathrm{F} \text { ) }\end{array}$ & $11,143,197$ & $21,649,320$ & $1,325,921$ & 143,003 & $1,251,282$ & 13,320 & $3,450,043$ \\
\hline
\end{tabular}


TABLE 4.5 Descriptions of Streams of Glycol Process for $\mathrm{H}_{2} \mathrm{~S}$ Removal in Case 1

\begin{tabular}{ccc}
\hline $\begin{array}{c}\text { Stream and } \\
\text { Characteristics }\end{array}$ & Data & Comments on Stream Calculations \\
\hline
\end{tabular}

Stream 1K: Synthesis gas from

shift system

Temperature $\left({ }^{\circ} \mathrm{F}\right)$

Pressure (psia)

Flow rate $(\mathrm{lb} \cdot \mathrm{mol} / \mathrm{h})$

$\mathrm{CO}_{2}$ (mole fraction)

$\mathrm{H}_{2} \mathrm{~S}$ (mole fraction)

Stream 1R: Feed gas to absorber

Temperature ( $\left.{ }^{\circ} \mathrm{F}\right)$

Pressure (psia)

Flow rate $(\mathrm{lb} \cdot \mathrm{mol} / \mathrm{h})$

$\mathrm{CO}_{2}$ (mole fraction)

$\mathrm{H}_{2} \mathrm{~S}$ (mole fraction)

Stream 2: Sulfur-free gases from absorber

Temperature ( $\left.{ }^{\circ} \mathrm{F}\right)$

Pressure (psia)

Flow rate (ib. $\mathrm{mol} / \mathrm{h}$ )

$\mathrm{CO}_{2}$ (mole fraction)

$\mathrm{H}_{2} \mathrm{~S}$ (mole fraction)
Stream 3: Lean glycol solvent

absorber

Pressure (psia)

Flow rate $(\mathrm{lb} \cdot \mathrm{mol} / \mathrm{h})$

$\mathrm{CO}_{2}$ (mole fraction)

$\mathrm{H}_{2} \mathrm{~S}$ (mole fraction)
The synthesis gas is shifted to maximize the overall $\mathrm{CO}_{2}$ recovery. After the shift, the gases are cooled to a temperature of $100^{\circ} \mathrm{F}$.

The shifted gases are cooled against the sulfur-free gas from the absorber to a temperature of $63^{\circ} \mathrm{F}$ in order to decrease the solvent circulation rate.

The composition of this stream corresponds to an $\mathrm{H}_{2} \mathrm{~S}$-removal efficiency of $99 \%$. Also, other gases like $\mathrm{CO}_{2}, \mathrm{COS}$, and $\mathrm{H}_{2}$ are absorbed by the solvent. The temperature of this stream is close to the temperature of lean solvent entering the absorber at the top.

Lean glycol solvent contains residual $\mathrm{H}_{2} \mathrm{~S}$ and $\mathrm{CO}_{2}$. The solvent also contains $30 \%$ water. $100 \%$ excess solvent is used.

Stream 4A: Rich glycol solvent from absorber

Temperature $\left({ }^{\circ} \mathrm{F}\right)$

Pressure (psia)

63.62 Flow rate reflects lean glycol solvent plus

Flow rate $(\mathrm{lb} \cdot \mathrm{mol} / \mathrm{h})$

$\mathrm{CO}_{2}$ (mole fraction)

446

$4,078.38$ absorbed $\mathrm{CO}_{2}, \mathrm{H}_{2} \mathrm{~S}$, and other gases. The

0.1735

$\mathrm{H}_{2} \mathrm{~S}$ (mole fraction)

0.0207 temperature rises because of the heat of absorption of $\mathrm{CO}_{2}$ and $\mathrm{H}_{2} \mathrm{~S}$. 


\begin{tabular}{ccc}
\hline $\begin{array}{c}\text { Stream and } \\
\text { Characteristics }\end{array}$ & Data & Comments on Stream Calculations \\
\hline
\end{tabular}

Stream 4B: Rich glycol solvent from turbine 1

Temperature $\left({ }^{\circ} \mathrm{F}\right)$

Pressure (psia)

Flow rate $(\mathrm{lb} \cdot \mathrm{mol} / \mathrm{h})$

$\mathrm{CO}_{2}$ (mole fraction)

$\mathrm{H}_{2} \mathrm{~S}$ (mole fraction)

Stream 5: Flash gas

Temperature $\left({ }^{\circ} \mathrm{F}\right)$

Pressure (psia)

Flow rate $(\mathrm{lb} \cdot \mathrm{mol} / \mathrm{h})$

$\mathrm{CO}_{2}$ (mole fraction)

$\mathrm{H}_{2} \mathrm{~S}$ (mole fraction)

Stream 6A: Rich glycol solvent to low-pressure power recovery turbine

Temperature $\left({ }^{\circ} \mathrm{F}\right)$

Pressure (psia)

Flow rate $(\mathrm{lb} \cdot \mathrm{mol} / \mathrm{h})$

$\mathrm{CO}_{2}$ (mole fraction)

$\mathrm{H}_{2} \mathrm{~S}$ (mole fraction)

Stream 6B: Rich glycol solvent from low-pressure power recovery turbine

Temperature $\left({ }^{\circ} \mathrm{F}\right)$

Pressure (psia)

Flow rate $(\mathrm{lb} \cdot \mathrm{mol} / \mathrm{h})$

$\mathrm{CO}_{2}$ (mole fraction)

$\mathrm{H}_{2} \mathrm{~S}$ (mole fraction)
62.68

100

$4,078.38$

0.1735

0.0207

42.44

100

610.96

0.9265

0.0007

42.44

100

$3,467.42$

0.0408

0.0231

42.10

14.7

$3,467.42$

0.0408

0.0231
This stream is exit stream from highpressure power recovery turbine. Exit pressure has been selected to avoid release of $\mathrm{H}_{2} \mathrm{~S}$ and $\mathrm{CO}_{2}$ while allowing some recovery of work of pressurization. The change in temperature over the turbine is estimated from change in enthalpy, which is taken to be equal to flow work.

$\mathrm{CO}_{2}$ and $\mathrm{H}_{2} \mathrm{~S}$ are released from the glycol solvent in the slump tank. This stream is not recycled to the absorber. The released gases contain mostly $\mathrm{CO}_{2}(93 \%)$ and therefore can be disposed of.

Change in composition simply reflects flashing of fuel gases to stream 5 .

This stream is exit stream from low-pressure turbine. The change in temperature is calculated as in $4 \mathrm{~B}$. 


\begin{tabular}{ccc} 
Stream and & & \\
Characteristics & Data & Comments on Stream Calculations \\
\hline
\end{tabular}

Stream 7: Rich glycol solvent to stripper

Temperature $\left({ }^{\circ} \mathrm{F}\right)$

Pressure (psia)

Flow rate $(\mathrm{lb} \cdot \mathrm{mol} / \mathrm{h})$

$\mathrm{CO}_{2}$ (mole fraction)

$\mathrm{H}_{2} \mathrm{~S}$ (mole fraction)

Stream 8: Lean glycol solvent from stripper

Temperature $\left({ }^{\circ} \mathrm{F}\right)$

Pressure (psia)

Flow rate $(\mathrm{lb} \cdot \mathrm{mol} / \mathrm{h})$

$\mathrm{CO}_{2}$ (mole fraction)

$\mathrm{H}_{2} \mathrm{~S}$ (mole fraction)

Stream 9: Lean glycol solvent from circulation pump

Temperature $\left({ }^{\circ} \mathrm{F}\right)$

Pressure (psia)

Flow rate $(\mathrm{lb} \cdot \mathrm{mol} / \mathrm{h})$

$\mathrm{CO}_{2}$ (mole fraction)

$\mathrm{H}_{2} \mathrm{~S}$ (mole fraction)

Stream 10: Lean glycol solvent after lean-rich solvent heat

exchanger

Temperature $\left({ }^{\circ} \mathrm{F}\right)$

Pressure (psia)

Flow rate $(\mathrm{lb} \cdot \mathrm{mol} / \mathrm{h})$

$\mathrm{CO}_{2}$ (mole fraction)

$\mathrm{H}_{2} \mathrm{~S}$ (mole fraction)

Stream 11: $\mathrm{H}_{2} \mathrm{~S}$-rich gas from

stripper

Temperature $\left({ }^{\circ} \mathrm{F}\right)$

Pressure (psia)

Flow rate $(\mathrm{lb} \cdot \mathrm{mol} / \mathrm{h})$

$\mathrm{CO}_{2}$ (mole fraction)

$\mathrm{H}_{2} \mathrm{~S}$ (mole fraction)

190 Rich glycol solvent is heated from $42.1^{\circ} \mathrm{F}$

14.7 to $190^{\circ} \mathrm{F}$ in lean-rich solvent heat

3,467.42 exchanger to decrease reboiler load.

212

14.7

$3,355.30$

0.0326

0.0065

215.21

451

$3,355.30$

0.0326

0.0065

66.87 Lean solvent is cooled against rich solvent

451 from the absorber to temperature of $67^{\circ} \mathrm{F}$

$3,355.30$

0.0326

0.0065

212

14.7

$1,134.41$

0.0282

0.0514
$\mathrm{CO}_{2}$ and $\mathrm{H}_{2} \mathrm{~S}$ are stripped from the solvent by heat. Stripper is operated at a temperature of $212^{\circ} \mathrm{F}$ and a pressure of 14.7 psia.

Lean glycol solvent from the stripper is at a pressure of $14.7 \mathrm{psia}$ and is pressurized to absorber pressure of 451 psia by circulation pump. The slight increase in temperature is due to work of compression.
The solubilities of gases decrease with temperature and therefore are released from the solvent. The composition of this stream represents amount of gases released and water evaporated. 


\begin{tabular}{|c|c|c|}
\hline $\begin{array}{l}\text { Stream and } \\
\text { Characteristics }\end{array}$ & Data & Comments on Stream Calculations \\
\hline \multicolumn{3}{|l|}{$\begin{array}{l}\text { Stream 12: } \mathrm{H}_{2} \mathrm{~S} \text {-rich gas after } \\
\text { condenser }\end{array}$} \\
\hline Temperature $\left({ }^{\circ} \mathrm{F}\right)$ & 100 & Mostly water is condensed in heat \\
\hline Pressure (psia) & 14.7 & exchanger by using cooling water to a \\
\hline Flow rate $(\mathrm{lb} \cdot \mathrm{mol} / \mathrm{h})$ & $1,134.41$ & temperature of $100^{\circ} \mathrm{F}$ \\
\hline $\mathrm{CO}_{2}$ (mole fraction) & 0.0282 & \\
\hline $\mathrm{H}_{2} \mathrm{~S}$ (mole fraction) & 0.0514 & \\
\hline \multicolumn{3}{|c|}{ Stream 13: $\mathrm{H}_{2} \mathrm{~S}$-product stream } \\
\hline Temperature ( $\left.{ }^{\circ} \mathrm{F}\right)$ & 100 & The gases are separated in the phase \\
\hline Pressure (psia) & 14.7 & separator. The gases are sent to Claus \\
\hline Flow rate $(\mathrm{lb} \cdot \mathrm{mol} / \mathrm{h})$ & 101.24 & plant for further treatment. \\
\hline $\mathrm{CO}_{2}$ (mole fraction) & 0.3159 & \\
\hline $\mathrm{H}_{2} \mathrm{~S}$ (mole fraction) & 0.5756 & \\
\hline \multicolumn{3}{|c|}{ Stream 14A: Recycle to stripper } \\
\hline Temperature $\left({ }^{\circ} \mathrm{F}\right)$ & 100 & To maintain low partial pressures of $\mathrm{H}_{2} \mathrm{~S}$ \\
\hline Pressure (psia) & 14.7 & and $\mathrm{CO}_{2}$, condensed water is recycled to \\
\hline Flow rate $(\mathrm{lb} \cdot \mathrm{mol} / \mathrm{h})$ & $1,022.29$ & the stripper. This also maintains the water \\
\hline $\mathrm{CO}_{2}$ (mole fraction) & 0.0000 & balance in the solvent. \\
\hline $\mathrm{H}_{2} \mathrm{~S}$ (mole fraction) & 0.0000 & \\
\hline \multicolumn{3}{|l|}{$\begin{array}{l}\text { Stream 14B: Wastewater for } \\
\text { treatment }\end{array}$} \\
\hline Temperature $\left({ }^{\circ} \mathrm{F}\right)$ & 100 & Excess water is removed through this \\
\hline Pressure (psia) & 14.7 & stream. \\
\hline Flow rate $(\mathrm{lb} \cdot \mathrm{mol} / \mathrm{h})$ & 10.88 & \\
\hline $\mathrm{CO}_{2}$ (mole fraction) & 0.0000 & \\
\hline $\mathrm{H}_{2} \mathrm{~S}$ (mole fraction) & 0.0000 & \\
\hline \multicolumn{3}{|c|}{$\begin{array}{l}\text { Stream 15A: Sulfur-free fuel gas } \\
\text { after heat exchange }\end{array}$} \\
\hline Temperature $\left({ }^{\circ} \mathrm{F}\right)$ & 70 & The fuel gas from the absorber is heated \\
\hline Pressure (psia) & 446 & against the feed to the absorber. These \\
\hline Flow rate $(\mathrm{lb} \cdot \mathrm{mol} / \mathrm{h})$ & $11,771.88$ & gases are further treated in $\mathrm{CO}_{2}$-removal \\
\hline $\mathrm{CO}_{2}$ (mole fraction) & 0.3661 & section. \\
\hline $\mathrm{H}_{2} \mathrm{~S}$ (mole fraction) & 0.0001 & \\
\hline
\end{tabular}




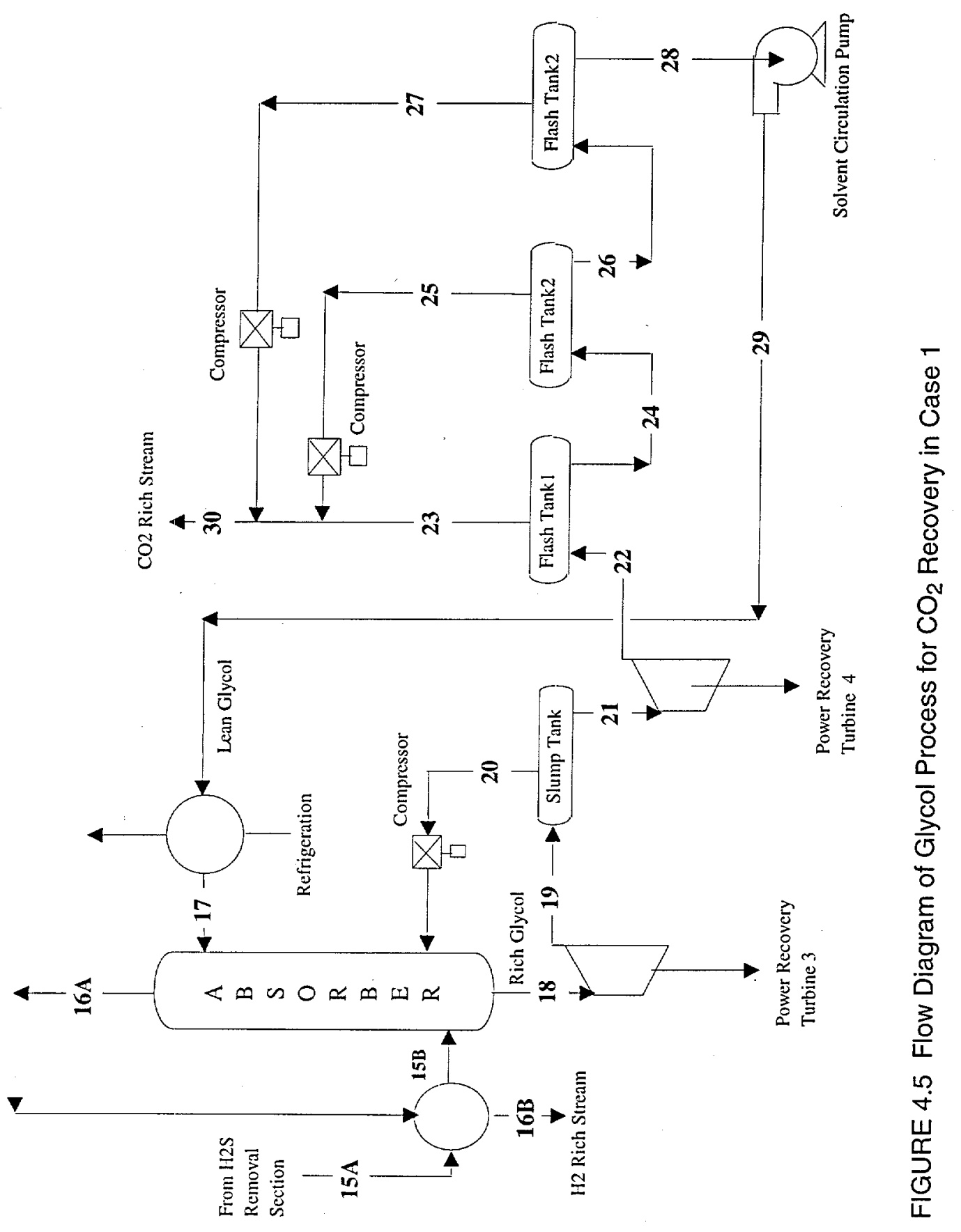


TABLE 4.6 Stream Flows of Glycol Process for $\mathrm{CO}_{2}$ Removal in Case 1

\begin{tabular}{|c|c|c|c|c|c|c|c|}
\hline Stream Data & Stream 15A & Stream 15B & Stream 16A & Stream 16B & Stream 17 & Stream 18 & Stream 15A \\
\hline Description of stream & $\begin{array}{l}\text { Sulfur-free feed } \\
\text { gas from } \mathrm{H}_{2} \mathrm{~S} \\
\text { removal section }\end{array}$ & Absorber feed & $\begin{array}{l}\text { Fuel gas from } \\
\text { absorber }\end{array}$ & $\begin{array}{l}\text { Fuel gas after } \\
\text { heat exchanger }\end{array}$ & $\begin{array}{l}\text { Lean glycol } \\
\text { solvent }\end{array}$ & $\begin{array}{l}\text { Rich glycol } \\
\text { solvent from } \\
\text { absorber }\end{array}$ & $\begin{array}{l}\text { Sulfur-free fuel } \\
\text { gas after heat } \\
\text { exchange }\end{array}$ \\
\hline \multicolumn{8}{|l|}{ Gases $(\mathrm{lb} \cdot \mathrm{mol} / \mathrm{h})$} \\
\hline $\mathrm{CO}$ & 45.13 & 45.13 & 44.68 & 44.68 & 0.00 & 4.51 & 45.13 \\
\hline $\mathrm{CO}_{2}$ & $4,310.02$ & $4,310.02$ & 43.10 & 43.10 & 118.16 & $4,474.57$ & $4,310.02$ \\
\hline $\mathrm{H}_{2}$ & $6,822.47$ & $6,822.47$ & $6,773.42$ & $6,773.42$ & 59.08 & $1,081.28$ & $6,822.47$ \\
\hline $\mathrm{H}_{2} \mathrm{O}$ & 0.00 & 0.00 & 0.00 & 0.00 & 0.00 & 0.00 & 0.00 \\
\hline $\mathrm{N}_{2}$ & 35.71 & 35.71 & 34.99 & 34.99 & 0.00 & 7.14 & 35.71 \\
\hline $\operatorname{Ar}$ & 72.72 & 72.72 & 72.72 & 72.72 & 0.00 & 0.00 & 72.72 \\
\hline $\mathrm{CH}_{4}$ & 463.02 & 463.02 & 439.87 & 439.87 & 0.00 & 154.34 & 463.02 \\
\hline $\mathrm{NH}_{3}$ & 18.79 & 18.79 & 18.79 & 18.79 & 0.00 & 0.00 & 18.79 \\
\hline $\mathrm{H}_{2} \mathrm{~S}$ & 0.63 & 0.63 & 0.00 & 0.00 & 7.38 & 8.09 & 0.63 \\
\hline $\mathrm{HCl}$ & 0.41 & 0.41 & 0.41 & 0.41 & 0.00 & 0.00 & 0.41 \\
\hline $\mathrm{O}_{2}$ & 0.00 & 0.00 & 0.00 & 0.00 & 0.00 & 0.00 & 0.00 \\
\hline $\cos$ & 2.97 & 2.97 & 1.19 & 1.19 & 0.00 & 3.57 & 2.97 \\
\hline $\mathrm{SO}_{2}$ & 0.00 & 0.00 & 0.00 & 0.00 & 0.00 & 0.00 & 0.00 \\
\hline Total gas flow & $11,771.88$ & $11,771.88$ & $7,429.18$ & $7,429.18$ & 184.62 & $5,733.51$ & $11,771.88$ \\
\hline \multicolumn{8}{|l|}{ Liquids $(\mathrm{lb} \cdot \mathrm{mol} / \mathrm{h}$ ) } \\
\hline Glycol solvent & 0.00 & 0.00 & 0.00 & 0.00 & $11,815.53$ & $11,815.53$ & 0.00 \\
\hline Temperature $\left({ }^{\circ} \mathrm{F}\right)$ & 70 & 55.00 & 30.00 & 56.24 & 30.00 & 61.97 & 70.00 \\
\hline Pressure (psia) & 446 & 446.00 & 441.00 & 441.00 & 446.00 & 441.00 & 446.00 \\
\hline $\begin{array}{l}\text { Enthalpy }(\mathrm{Btu} / \mathrm{h}) \\
\text { (reference, } 32^{\circ} \mathrm{F} \text { ) }\end{array}$ & $3,450,043$ & $2,083,999$ & $-103,990$ & $1,262,022$ & $-3,245,210$ & $50,053,110$ & $3,450,043$ \\
\hline
\end{tabular}


TABLE 4.6 (Cont.)

\begin{tabular}{|c|c|c|c|c|c|c|}
\hline Stream Data & Stream 19 & Stream 20 & Stream 21 & Stream 22 & Stream 23 & Stream 24 \\
\hline Description of stream & $\begin{array}{l}\text { Rich glycol solvent } \\
\text { after turbine } 3\end{array}$ & $\begin{array}{l}\text { Recycle to } \\
\text { absorber }\end{array}$ & $\begin{array}{l}\text { Rich glycol solvent } \\
\text { to turbine } 4\end{array}$ & $\begin{array}{l}\text { Rich glycol solvent } \\
\text { after turbine } 4\end{array}$ & $\begin{array}{l}\mathrm{CO}_{2} \text {-rich gas } \\
\text { from 1st flash }\end{array}$ & $\begin{array}{l}\text { Rich glycol soivent } \\
\text { to } 2 \text { nd flash }\end{array}$ \\
\hline \multicolumn{7}{|l|}{ Gases $(\mathrm{lb} \cdot \mathrm{mol} / \mathrm{h})$} \\
\hline $\mathrm{CO}$ & 4.51 & 4.06 & 0.45 & 0.45 & 0.45 & 0.00 \\
\hline $\mathrm{CO}_{2}$ & $4,474.57$ & 89.49 & $4,385.08$ & $4,385.08$ & $3,288.81$ & $1,096.27$ \\
\hline $\mathrm{H}_{2}$ & $1,081.28$ & 973.16 & 108.13 & 108.13 & 32.44 & 75.69 \\
\hline $\mathrm{H}_{2} \mathrm{O}$ & 0.00 & 0.00 & 0.00 & 0.00 & 0.00 & 0.00 \\
\hline $\mathrm{N}_{2}$ & 7.14 & 6.43 & 0.71 & 0.71 & 0.71 & 0.00 \\
\hline $\mathrm{Ar}$ & 0.00 & 0.00 & 0.00 & 0.00 & 0.00 & 0.00 \\
\hline $\mathrm{CH}_{4}$ & 154.34 & 131.19 & 23.15 & 23.15 & 18.52 & 4.63 \\
\hline $\mathrm{NH}_{3}$ & 0.00 & 0.00 & 0.00 & 0.00 & 0.00 & 0.00 \\
\hline $\mathrm{H}_{2} \mathrm{~S}$ & 8.09 & 0.08 & 8.01 & 8.01 & 0.08 & 7.93 \\
\hline $\mathrm{HCl}$ & 0.00 & 0.00 & 0.00 & 0.00 & 0.00 & 0.00 \\
\hline $\mathrm{O}_{2}$ & 0.00 & 0.00 & 0.00 & 0.00 & 0.00 & 0.00 \\
\hline $\cos$ & 3.57 & 1.78 & 1.78 & 1.78 & 0.45 & 1.34 \\
\hline $\mathrm{SO}_{2}$ & 0.00 & 0.00 & 0.00 & 0.00 & 0.00 & 0.00 \\
\hline Total gas flow & $5,733.51$ & $1,206.19$ & $4,527.32$ & $4,527.32$ & $3,341.46$ & $1,185.86$ \\
\hline \multicolumn{7}{|l|}{ Liquids $(\mathrm{lb} \cdot \mathrm{mol} / \mathrm{h})$} \\
\hline Glycol solvent & $11,815.53$ & 0.00 & $11,815.53$ & $11,815.53$ & 0.00 & $11,815.53$ \\
\hline Temperature $\left({ }^{\circ} \mathrm{F}\right)$ & 60.99 & 60.38 & 60.38 & 59.76 & 37.26 & 37.26 \\
\hline Pressure (psia) & 200.00 & 200.00 & 200.00 & 50.00 & 50.00 & 50.00 \\
\hline $\begin{array}{l}\text { Enthalpy }(\mathrm{Btu} / \mathrm{h}) \\
\left(\text { reference, } 32^{\circ} \mathrm{F}\right)\end{array}$ & $48,408,324$ & 246,412 & $47,144,244$ & $46,116,263$ & 155,492 & $8,587,255$ \\
\hline
\end{tabular}


TABLE 4.6 (Cont.)

\begin{tabular}{|c|c|c|c|c|c|c|}
\hline Stream Data & Stream 25 & Stream 26 & Stream 27 & Stream 28 & Stream 29 & Stream 30 \\
\hline Description of stream & $\begin{array}{l}\mathrm{CO}_{2} \text {-rich gas from } \\
\text { 2nd flash }\end{array}$ & $\begin{array}{l}\text { Rich glycol solvent } \\
\text { to 3rd flash }\end{array}$ & $\begin{array}{l}\mathrm{CO}_{2} \text {-rich gas from } \\
\text { 3rd flash }\end{array}$ & Lean glycol solvent & $\begin{array}{l}\text { Lean glycol solvent } \\
\text { after circulation pump }\end{array}$ & $\mathrm{CO}_{2}$-rich product \\
\hline \multicolumn{7}{|l|}{ Gases (lb.mol/h) } \\
\hline CO & 0.00 & 0.00 & 0.00 & 0.00 & 0.00 & 0.45 \\
\hline $\mathrm{CO}_{2}$ & 767.39 & 328.88 & 210.47 & 118.41 & 118.41 & $4,266.66$ \\
\hline $\mathrm{H}_{2}$ & 15.14 & 60.55 & 1.37 & 59.18 & 59.18 & 48.94 \\
\hline $\mathrm{H}_{2} \mathrm{O}$ & 0.00 & 0.00 & 0.00 & 0.00 & 0.00 & 0.00 \\
\hline $\mathrm{N}_{2}$ & 0.00 & 0.00 & 0.00 & 0.00 & 0.00 & 0.71 \\
\hline $\mathrm{Ar}$ & 0.00 & 0.00 & 0.00 & 0.00 & 0.00 & 0.00 \\
\hline $\mathrm{CH}_{4}$ & 4.63 & 0.00 & 0.00 & 0.00 & 0.00 & 23.15 \\
\hline $\mathrm{NH}_{3}$ & 0.00 & 0.00 & 0.00 & 0.00 & 0.00 & 0.00 \\
\hline $\mathrm{H}_{2} \mathrm{~S}$ & 0.40 & 7.53 & 0.14 & 7.39 & 7.39 & 0.62 \\
\hline $\mathrm{HCl}$ & 0.00 & 0.00 & 0.00 & 0.00 & 0.00 & 0.00 \\
\hline $\mathrm{O}_{2}$ & 0.00 & 0.00 & 0.00 & 0.00 & 0.00 & 0.00 \\
\hline $\cos$ & 1.34 & 0.00 & 0.00 & 0.00 & 0.00 & 1.78 \\
\hline $\mathrm{SO}_{2}$ & 0.00 & 0.00 & 0.00 & 0.00 & 0.00 & 0.00 \\
\hline Total gas flow & 788.89 & 396.97 & 211.98 & 184.99 & 184.99 & $4,342.33$ \\
\hline \multicolumn{7}{|l|}{ Liquids (lb·mol/h) } \\
\hline Glycol solvent & 0.00 & $11,815.53$ & 0.00 & $11,815.53$ & $11,815.53$ & 0.00 \\
\hline Temperature $\left({ }^{\circ} \mathrm{F}\right)$ & 31.92 & 31.92 & 30.44 & 30.44 & 33.90 & 81.53 \\
\hline Pressure (psia) & 14.70 & 14.70 & 4.00 & 4.00 & 446.00 & 50.00 \\
\hline $\begin{array}{l}\text { Enthalpy }(B t u / h) \\
\text { (reference, } 32^{\circ} \mathrm{F} \text { ) }\end{array}$ & -580 & $-135,628$ & $-2,916$ & $-2,525,525$ & $3,075,331$ & $1,922,357$ \\
\hline
\end{tabular}


TABLE 4.7 Descriptions of Streams of Glycol Process for $\mathrm{CO}_{2}$ Removal in Case 1

\begin{tabular}{ccc} 
Stream and & & \\
Characteristics & Data & Comments on Stream Calculations \\
\hline
\end{tabular}

Stream 15A: Sulfur-free gas from

$\mathrm{H}_{2} \mathrm{~S}$ section

Temperature $\left({ }^{\circ} \mathrm{F}\right)$

Pressure (psia)

Flow rate $(\mathrm{lb} \cdot \mathrm{mol} / \mathrm{h})$

$\mathrm{CO}_{2}$ (mole fraction)

$\mathrm{H}_{2} \mathrm{~S}$ (mole fraction)

Stream 15B: Feed gas to absorber

Temperature $\left({ }^{\circ} \mathrm{F}\right)$

Pressure (psia)

Flow rate $(\mathrm{lb} \cdot \mathrm{mol} / \mathrm{h})$

$\mathrm{CO}_{2}$ (mole fraction)

$\mathrm{H}_{2} \mathrm{~S}$ (mole fraction)

Stream 16A: Fuel gas from

absorber

Temperature $\left({ }^{\circ} \mathrm{F}\right)$

Pressure (psia)

Flow rate $(\mathrm{lb} \cdot \mathrm{mol} / \mathrm{h})$

$\mathrm{CO}_{2}$ (mole fraction)

$\mathrm{H}_{2} \mathrm{~S}$ (mole fraction)

Stream 16B: Fuel gas after

heat exchanger

Temperature $\left({ }^{\circ} \mathrm{F}\right)$

Pressure (psia)

Flow rate $(\mathrm{lb} \cdot \mathrm{mol} / \mathrm{h})$

$\mathrm{CO}_{2}$ (mole fraction)

$\mathrm{H}_{2} \mathrm{~S}$ (mole fraction)

Stream 17: Lean glycol to the of absorber

Temperature ( $\left.{ }^{\circ} \mathrm{F}\right)$

Pressure (psia)

Flow rate $(\mathrm{lb} \cdot \mathrm{mol} / \mathrm{h})$

$\mathrm{CO}_{2}$ (mole fraction)

$\mathrm{H}_{2} \mathrm{~S}$ (mole fraction)
70 The synthesis gas is cleaned in two

446 stages. First sulfur compounds are

$11,771.88$ removed. Then they are fed to another

0.3661 absorption column for $\mathrm{CO}_{2}$ recovery.

0.0001

55

446

$11,771.88$

0.3661

0.0001

30

441

$7,429.18$

0.0058

0.0000

56.24

441

$7,429.18$

0.0058

0.0000

30

446

$12,000.15$

0.0098

0.0006
The sulfur-free synthesis gas is cooled against the cold fuel gas from top of the absorber to a temperature of $55^{\circ} \mathrm{F}$.

The composition of this stream corresponds to a $\mathrm{CO}_{2}$-removal efficiency of $99 \%$. Also, other gases like $\mathrm{H}_{2} \mathrm{~S}, \mathrm{COS}$, and $\mathrm{H}_{2}$ are absorbed by the solvent. The temperature of this stream is close to the temperature of lean solvent entering the absorber at the top.

Fuel gas is heated against the sulfurfree gases from $\mathrm{H}_{2} \mathrm{~S}$ section.

Lean glycol solvent contains residual $\mathrm{CO}_{2}$ and $\mathrm{H}_{2} \mathrm{~S} .50 \%$ excess solvent is used. The solvent is cooled to $30^{\circ} \mathrm{F}$ by refrigeration. 
TABLE 4.7 (Cont.)

\begin{tabular}{ccc}
\hline $\begin{array}{c}\text { Stream and } \\
\text { Characteristics }\end{array}$ & Data & Comments on Stream Calculations \\
\hline
\end{tabular}

Stream 18: Rich glycol solvent

from absorber

Temperature $\left({ }^{\circ} \mathrm{F}\right)$

Pressure (psia)

Flow rate $(\mathrm{lb} \cdot \mathrm{mol} / \mathrm{h})$

$\mathrm{CO}_{2}$ (mole fraction)

$\mathrm{H}_{2} \mathrm{~S}$ (mole fraction)

Stream 19: Rich glycol solvent

from turbine 3

Temperature $\left({ }^{\circ} \mathrm{F}\right)$

Pressure (psia)

Flow rate $(\mathrm{lb} \cdot \mathrm{mol} / \mathrm{h})$

$\mathrm{CO}_{2}$ (mole fraction)

$\mathrm{H}_{2} \mathrm{~S}$ (mole fraction)
61.97 Flow rate reflects lean glycol solvent plus

441 absorbed $\mathrm{CO}_{2}, \mathrm{H}_{2} \mathrm{~S}$, and other gases. The

$17,549.04$ temperature rises because of the heat of

0.2550 absorption of $\mathrm{CO}_{2}$ and $\mathrm{H}_{2} \mathrm{~S}$.
60.99

200

$17,549.04$

0.2550

0.0005

60.38

200

$1,206.19$

0.0742

0.0001

Stream 21: Rich glycol solvent to low-pressure power recovery turbine

Temperature $\left({ }^{\circ} \mathrm{F}\right)$

Pressure (psia)

Flow rate $(\mathrm{lb} \cdot \mathrm{mol} / \mathrm{h})$

$\mathrm{CO}_{2}$ (mole fraction)

$\mathrm{H}_{2} \mathrm{~S}$ (mole fraction)

Stream 22: Rich glycol solvent from low-pressure power recovery turbine

Temperature $\left({ }^{\circ} \mathrm{F}\right)$

Pressure (psia)

Flow rate $(\mathrm{lb} \cdot \mathrm{mol} / \mathrm{h})$

$\mathrm{CO}_{2}$ (mole fraction)

$\mathrm{H}_{2} \mathrm{~S}$ (mole fraction)
60.38

200

$16,342.85$

0.2683

0.0005

59.76

0.0005
This stream is exit stream from highpressure power recovery turbine. Exit pressure has been selected to avoid release of $\mathrm{CO}_{2}$ and $\mathrm{H}_{2} \mathrm{~S}$ while allowing some recovery of work of pressurization. The change in temperature over the turbine is estimated from change in enthalpy, which is taken to be equal to flow work.

$\mathrm{CO}_{2}$ and $\mathrm{H}_{2} \mathrm{~S}$ are released from the glycol solvent in the slump tank. This stream is compressed and recycled to the absorber to decrease the losses of valuable gases like $\mathrm{H}_{2}$ and $\mathrm{CO}$.

Change in composition simply reflects flashing of fuel gases to stream 20.

This stream is exit from low-pressure turbine. The change in temperature is calculated as in stream 19. 


\begin{tabular}{ccc}
\hline Stream and & & \\
Characteristics & Data & Comments on Stream Calculations \\
\hline
\end{tabular}

Stream 23: $\mathrm{CO}_{2}$-rich flash gas from high-pressure flash tank

Temperature $\left({ }^{\circ} \mathrm{F}\right)$

Pressure (psia)

Flow rate $(\mathrm{lb} \cdot \mathrm{mol} / \mathrm{h})$

$\mathrm{CO}_{2}$ (mole fraction)

$\mathrm{H}_{2} \mathrm{~S}$ (mole fraction)

Stream 24: Glycol solvent from

high-pressure flash tank

Temperature $\left({ }^{\circ} \mathrm{F}\right)$

Pressure (psia)

Flow rate $(\mathrm{lb} \cdot \mathrm{mol} / \mathrm{h})$

$\mathrm{CO}_{2}$ (mole fraction)

$\mathrm{H}_{2} \mathrm{~S}$ (mole fraction)

Stream 25: $\mathrm{CO}_{2}$-rich flash gas from intermediate-pressure flash tank

Temperature $\left({ }^{\circ} \mathrm{F}\right)$

Pressure (psia)

Flow rate $(\mathrm{lb} \cdot \mathrm{mol} / \mathrm{h})$

$\mathrm{CO}_{2}$ (mole fraction)

$\mathrm{H}_{2} \mathrm{~S}$ (mole fraction)

Stream 26: Glycol solvent from intermediate-pressure flash tank

Temperature $\left({ }^{\circ} \mathrm{F}\right)$

Pressure (psia)

Flow rate $(\mathrm{lb} \cdot \mathrm{mol} / \mathrm{h})$

$\mathrm{CO}_{2}$ (mole fraction)

$\mathrm{H}_{2} \mathrm{~S}$ (mole fraction)

Stream 27: $\mathrm{CO}_{2}$-rich flash gas from low-pressure flash tank

Temperature $\left({ }^{\circ} \mathrm{F}\right)$

Pressure (psia)

Flow rate $(\mathrm{lb} \cdot \mathrm{mol} / \mathrm{h})$

$\mathrm{CO}_{2}$ (mole fraction)

$\mathrm{H}_{2} \mathrm{~S}$ (mole fraction)

37.26 The $\mathrm{CO}_{2}$ from the rich glycol solvent is

50 released in stages. In the first stage, the gases

$3,341.46$

0.9842

0.0000 are flashed to a pressure of $50 \mathrm{psia}$. The amount of $\mathrm{CO}_{2}$ remaining in the solvent depends on pressure, and the $\mathrm{CO}_{2}$ released is calculated by mass balance.

37.26

50

$13,001.39$

0.0843

0.0006

31.92

17.70

788.89

0.9727

0.0005

31.92

14.7

$12,212.50$

0.0269

0.0006

30.44 Glycol solvent is flashed to a pressure of

4.04 psia to remove as much $\mathrm{CO}_{2}$ as

211.98 possible. The lower residual amount of $0.9929 \mathrm{CO}_{2}$ in lean glycol solvent reduces the

0.0007 circulation rate of solvent.
The amount of $\mathrm{CO}_{2}$ in solvent and released as gas is calculated as in stream 23. Sufficient residence is provided for the gases to separate from solvent. 
TABLE 4.7 (Cont.)

\begin{tabular}{|c|c|c|}
\hline $\begin{array}{l}\text { Stream and } \\
\text { Characteristics }\end{array}$ & Data & Comments on Stream Calculations \\
\hline \multicolumn{3}{|c|}{$\begin{array}{l}\text { Stream 28: Lean glycol solvent from } \\
\text { low-pressure flash tank }\end{array}$} \\
\hline Temperature $\left({ }^{\circ} \mathrm{F}\right)$ & 30.44 & $\cdots$ \\
\hline Pressure (psia) & 4.0 & \\
\hline Flow rate $(\mathrm{lb} \cdot \mathrm{mol} / \mathrm{h})$ & $12,000.52$ & . \\
\hline $\mathrm{CO}_{2}$ (mole fraction) & 0.0098 & \\
\hline $\mathrm{H}_{2} \mathrm{~S}$ (mole fraction) & 0.0006 & \\
\hline \multicolumn{3}{|c|}{$\begin{array}{l}\text { Stream 29: Lean glycol solvent after } \\
\text { circulation pump }\end{array}$} \\
\hline Temperature $\left({ }^{\circ} \mathrm{F}\right)$ & 33.90 & The lean solvent is pressurized to the \\
\hline Pressure (psia) & 446 & absorber operating pressure by using a pump. \\
\hline Flow rate $(\mathrm{lb} \cdot \mathrm{mol} / \mathrm{h})$ & $12,000.52$ & The change in temperature is due to work \\
\hline $\mathrm{CO}_{2}$ (mole fraction) & 0.0098 & of compression. The solvent is chilled \\
\hline $\mathrm{H}_{2} \mathrm{~S}$ (mole fraction) & 0.0006 & before being sent to the absorber. \\
\hline \multicolumn{3}{|c|}{ Stream 30: $\mathrm{CO}_{2}$-rich product gas } \\
\hline Temperature $\left({ }^{\circ} \mathrm{F}\right)$ & 81.53 & Flash gases from intermediate- and low- \\
\hline Pressure (psia) & 50.0 & pressure flash tanks are compressed to the \\
\hline Flow rate $(\mathrm{lb} \cdot \mathrm{mol} / \mathrm{h})$ & $4,342.33$ & pressure of stream 23. Streams 23 \\
\hline $\mathrm{CO}_{2}$ (mole fraction) & 0.9826 & 25 , and 27 are combined for further \\
\hline $\mathrm{H}_{2} \mathrm{~S}$ (mole fraction) & 0.0001 & compression for pipeline. \\
\hline
\end{tabular}


TABLE 4.8 Power Output, Plant Power Use, and Net Power Output for Base Case and Case 1 Gas Turbine/Glycol Process

\begin{tabular}{lcc}
\hline & \multicolumn{2}{c}{ Power (MW) } \\
\cline { 2 - 3 } Power Variable & Base Case & Glycol Case \\
\hline Power output & & \\
Gas turbine & 298.8 & 284.8 \\
Steam turbine & 159.4 & 161.6 \\
& & \\
Internal power consumption & & \\
CO $\mathrm{O}_{2}$ recovery & & \\
CO & \\
Solvent circulation & 0 & -17.3 \\
Solvent refrigeration & 0 & -5.8 \\
Power recovery turbine & 0 & -4.5 \\
Gasification system & 0 & 3.4 \\
& -44.7 & -44.7 \\
Net power output & & \\
& 413.5 & 377.5 \\
Energy penalty & 0 & 36 \\
\hline
\end{tabular}

\subsection{Economics}

Details of the direct capital investment estimates for the $\mathrm{H}_{2} \mathrm{~S}$ recovery system, the shift system, and the $\mathrm{CO}_{2}$ recovery system are presented in Tables 4.9, 4.10, and 4.11, respectively. Total cost information, including indirect capital investment and operating and maintenance costs, is provided in Section 9. 
TABLE 4.9 Sizing and Cost Estimation for Major Equipment Used for $\mathrm{H}_{2} \mathrm{~S}$ Removal in Glycol Process in Case 1

1. Heat Exchanger before the Absorption Column

$\mathrm{Q}=$ Load (Btu/h)

Tha $=$ Inlet temperature of hot fluid $\left({ }^{\circ} \mathrm{F}\right)$

$3,630,585$

100

$\mathrm{Thb}=$ Outlet temperature of hot fluid $\left({ }^{\circ} \mathrm{F}\right)$

Pressure of hot gases (psia)

63

451

Tca $=$ Inlet temperature of cold fluid $\left({ }^{\circ} \mathrm{F}\right)$

$\mathrm{Tcb}=$ Outlet temperature of cold fluid $\left({ }^{\circ} \mathrm{F}\right) \quad 70$

Delta T1. 30

Delta T2 33

Log mean temperature difference $\left({ }^{\circ} \mathrm{F}\right)$

Overall heat transfer coefficient $\left(\mathrm{Btu} / \mathrm{h} / \mathrm{ft}^{2} /{ }^{\circ} \mathrm{F}\right)$

Heat transfer area $\left(\mathrm{ft}^{2}\right) \quad 23,070$

Operating Pressure (psia) $\quad 451$

$\begin{array}{ll}\text { Pressure factor } & 1.175\end{array}$

Materials correction factor 1

Module factor $\quad 3.2$

(includes all of the supporting equipment and connections and installation)

Purchased cost of heat exchanger in 1987

(mild steel construction; shell and tube floating head)

CE index for process equipment in 1987

$\$ 185,000$

CE index for process equipment in 1995

320

373.9

Installed cost of heat exchanger in 1995

$\$ 812,765$

2. $\mathrm{H}_{2} \mathrm{~S}$ Absorption Column

Diameter of tower ( $\mathrm{ft}$ )

HETP (ft)

No. of theoretical stages

Absorber tower height ( $\mathrm{ft}$ )

( $4 \mathrm{ft}$ for inlet, outlet and gas, and liquid distributors)

Volume of packing $\left(\mathrm{ft}^{3}\right)$

Pressure factor

1,810

Cost per foot of column height

(mild steel construction)

Materials correction factor

Module factor

4.16

CE index for process equipment in 1987

CE index for process equipment in 1995

Installed cost of absorber in 1995

Cost of packing per cubic foot

$\$ 63.5$

$\$ 505,513$

(2-in. pall rings-metal)

Total cost of packing 
TABLE 4.9 (Cont.)

3. Power Recovery Turbine 1

Turbine size (hp)

173

Purchased cost in 1987

$\$ 120,000$

Module factor

CE index for process equipment in 1987

CE index for process equipment in 1995

373.9

Installed cost of solvent pump in 1995

$\$ 175,266$

4. Slump Tank

Glycol solvent flow rate $(\mathrm{lb} / \mathrm{h})$

Density of glycol solvent (lb/gal)

613,374

8.6

Residence time (s)

180

Slump tank volume (gal)

3,566

Pressure factor

1

Materials correction factor

Module factor

2.08

Purchased cost of slump tank in 1987

(mild steel construction)

CE index for process equipment in 1987

$\$ 13,000$

CE index for process equipment in 1995

Installed cost of slump tank in 1995

373.9

5. Power Recovery Turbine 2

Turbine size (hp)

Purchased cost in 1987

43

$\$ 65,000$

Module factor

CE index for process equipment in 1987

373.9

Installed cost of solvent pump in 1995

$\$ 75,948$

6. Solvent Circulation Pump

Horsepower

Purchased cost of pump in 1987

(includes motor, coupling, base; cast iron, horizontal)

Materials correction factor

Module factor

1.5

320

CE index for process equipment in 1987

373.9

$\$ 31,595$

CE index for process equipment in 1995

Installed cost of solvent pump in 1995

$\$ 52,580$ 
7. Lean-Rich Solvent Heat Exchanger

$Q=\operatorname{Load}(B t u / h)$

Tha $=$ Inlet temperature of hot fluid $\left({ }^{\circ} \mathrm{F}\right)$

$47,724.550$

215.21

$\mathrm{Thb}=$ Outlet temperature of hot fluid $\left({ }^{\circ} \mathrm{F}\right)$

67

Pressure of hot gases (psia)

450

$\mathrm{Tca}=$ Inlet temperature of cold fluid $\left({ }^{\circ} \mathrm{F}\right)$

42.10

$\mathrm{Tcb}=$ Outlet temperature of cold fluid $\left({ }^{\circ} \mathrm{F}\right)$

190.00

Delta T1

Delta T2

25.2077

Log mean temperature difference $\left({ }^{\circ} \mathrm{F}\right)$

Overall heat transfer coefficient $\left(\mathrm{Btu} / \mathrm{h} / \mathrm{ft} 2 /{ }^{\circ} \mathrm{F}\right)$

Heat transfer area $\left(\mathrm{ft}^{2}\right)$

Operating pressure (psia)

12,697

Pressure factor

Materials correction factor

Module factor

(includes all of the supporting equipment and connections and

Purchased cost of heat exchanger in 1987

(mild steel construction; shell and tube floating head)

$\$ 120,000$

CE index for process equipment in 1987

CE index for process equipment in 1995

373.9

Installed cost of heat exchanger in 1995

\section{Stripping Column}

Diameter of tower ( $\mathrm{ft}$ )

HETP (ft)

No. of theoretical stages

Absorber tower height

(4 ft for inlet, outlet and gas, and liquid distributors)

Volume of packing $\left(\mathrm{ft}^{3}\right)$

Pressure factor

Materials correction factor (stainless steel 304)

Cost per foot of column height

(mild steel construction)

Module factor

CE index for process equipment in 1987

CE index for process equipment in 1995

(2-in. pall rings-SS)

Materials correction factor

$\$ 63.5$

Total cost of packing 
9. Overhead Condenser

$Q=\operatorname{Load}($ Btu/h)

$20,323,399$

Tha $=$ Inlet temperature of hot fluid $\left({ }^{\circ} \mathrm{F}\right)$

212.00

Thb $=$ Outlet temperature of hot fluid $\left({ }^{\circ} \mathrm{F}\right)$

100

Pressure of hot gases (psia)

14.7

$\mathrm{Tca}=$ Inlet temperature of cold fluid $\left({ }^{\circ} \mathrm{F}\right)$

70.00

$\mathrm{Tcb}=$ Outlet temperature of cold fluid $\left({ }^{\circ} \mathrm{F}\right)$

180.00

Delta T1

Delta T2

Log mean temperature difference $\left({ }^{\circ} \mathrm{F}\right)$

Overall heat transfer coefficient $\left(\mathrm{Btu} / \mathrm{h} / \mathrm{ft}^{2} /{ }^{\circ} \mathrm{F}\right)$

Heat transfer area $\left(\mathrm{ft}^{2}\right)$

Operating Pressure (psia)

16,396

Pressure factor

14.7

Materials correction factor 1 for SS

Materials correction factor 2 for SS

Module factor

(includes all of the supporting equipment and connections and installation)

Purchased cost of heat exchanger in 1987

(mild steel construction; shell and tube floating head)

CE index for process equipment in 1987

CE index for process equipment in 1995

$\$ 160,000$

Installed cost of heat exchanger in 1995

10. Phase Separator

Flow rate (lb/h)

22,291

0.04

Density of fluid (lb/gal)

phase separator volume (gal)

18,576

Pressure factor

Materials correction factor (stainless steel)

Module factor

2.08

Purchased cost of phase separator in 1987

(mild steel construction)

CE index for process equipment in 1987

CE index for process equipment in 1995 
TABLE 4.9 (Cont.)

11. Solvent Refrigeration

Refrigeration (tons)

Purchased cost in 1987

Temperature correction factor

Module factor

CE index for process equipment in 1987

CE index for process equipment in 1995

Installed cost of refrigeration in 1995

981.96

$\$ 400,000$

1.25

1.46

320

373.9

$\$ 852,959$

$\$ 5,918,829$

$\$ 17,756,488$ 
TABLE 4.10 Sizing and Cost Estimation for Major Equipment Used for Shift System in Case 1

1. First-Stage Shift Reactor

Catalyst volume $\left(\mathrm{ft}^{3}\right) \quad 665$

Reactor volume $\left(\mathrm{ft}^{3}\right)$ (1.2 times the catalyst volume) 798

Reactor volume (gal) $\quad 5,969$

Pressure factor $\quad 2.8$

Module factor $\quad 3.05$

CE index for process equipment in 1987

CE index for process equipment in 1995

Purchased cost of reactor in $1987 \quad \$ 7,000$

Installed cost of reactor in 1995

$\$ 69,849$

2. Second-Stage Shift Reactor

Catalyst volume $\left(\mathrm{ft}^{3}\right) \quad 285$

Reactor volume $\left(\mathrm{ft}^{3}\right)$ (1.2 times the catalyst volume) 342

$\begin{array}{ll}\text { Reactor volume (gal) } & 2,558\end{array}$

Pressure factor $\quad 2.8$

Module factor $\quad 3.05$

CE index for process equipment in 1987

CE index for process equipment in 1995

Purchased cost of reactor in $1987 \quad \$ 5,000$

Installed cost of reactor in 1995

$\$ 49,892$

3. Shift Catalyst

Volume of catalyst in first stage $\left(\mathrm{ft}^{3}\right) \quad 999$

Volume of catalyst in second stage $\left(\mathrm{ft}^{3}\right) \quad 339$

Cost of high-temperature catalyst per cubic foot $\quad \$ 50$

Cost of low-temperature catalyst per cubic foot $\quad \$ 250$

Total cost of catalyst

$\$ 134,647$ 
4. Heat Exchanger between First- and Second-Shift Stages

$Q=$ Load (Btu/h)

Tha $=$ Inlet temperature of hot fluid $\left({ }^{\circ} \mathrm{F}\right)$

$56,070,250$

684

457

Thb = Outlet temperature of hot fluid $\left({ }^{\circ} \mathrm{F}\right)$

451

$\mathrm{Tca}=$ Inlet temperature of cold fluid $\left({ }^{\circ} \mathrm{F}\right)$

400

$\mathrm{Tcb}=$ Outlet temperature of cold fluid $\left({ }^{\circ} \mathrm{F}\right)$

457

Delta T1

226

Delta T2

57

Log mean temperature difference $\left({ }^{\circ} \mathrm{F}\right)$

123

Overall heat transfer coefficient $\left(\mathrm{Btu} / \mathrm{h} / \mathrm{ft} \mathrm{t}^{2} /^{\circ} \mathrm{F}\right)$

Heat transfer area $\left(\mathrm{ft}^{2}\right)$

Operating pressure ( $\mathrm{psia}$ )

40

11,383

451

Pressure factor

1.175

Materials correction factor

Module factor

(includes all of the supporting equipment and connections and installation)

Purchased cost of heat exchanger in 1987

(mild steel construction; shell and tube floating head)

CE index for process equipment in 1987

CE index for process equipment in 1995

Installed cost of heat exchanger in 1995

5. Heat Exchanger after Second-Stage Shift for Raising Steam

$Q=\operatorname{Load}(B t u / h)$

Tha $=$ Inlet temperature of hot fluid $\left({ }^{\circ} \mathrm{F}\right)$

$\mathrm{Thb}=$ Outlet temperature of hot fluid $\left({ }^{\circ} \mathrm{F}\right)$

Pressure of hot gases (psia)

$\mathrm{Tca}=$ Inlet temperature of cold fluid $\left({ }^{\circ} \mathrm{F}\right)$

$\mathrm{Tcb}=$ Outlet temperature of cold fluid $\left({ }^{\circ} \mathrm{F}\right)$

Delta T1

Delta T2

Log mean temperature difference $\left({ }^{\circ} \mathrm{F}\right)$

Overall heat transfer coefficient $\left(\mathrm{Btu} / \mathrm{h} / \mathrm{ft}^{2} /{ }^{\circ} \mathrm{F}\right)$

Heat transfer area $\left(\mathrm{ft}^{2}\right)$

Operating pressure (psia)

Pressure factor

Materials correction factor

Module factor

(includes all of the supporting equipment and connections and installation)

Purchased cost of heat exchanger in 1987

(mild steel construction; shell and tube floating head)

CE index for process equipment in 1987

CE index for process equipment in 1995

Installed cost of heat exchanger in 1995 
6. Heat Exchanger after Second-Stage for Heating Fuel Gas

$Q=\operatorname{Load}(B t u / h)$

Tha $=$ Inlet temperature of hot fluid $\left({ }^{\circ} \mathrm{F}\right)$

$\mathrm{Thb}=$ Outlet temperature of hot fluid $\left({ }^{\circ} \mathrm{F}\right)$

Pressure of hot gases (psia)

$\mathrm{Tca}=$ Inlet temperature of cold fluid $\left({ }^{\circ} \mathrm{F}\right)$

$\mathrm{Tcb}=$ Outlet temperature of cold fluid $\left({ }^{\circ} \mathrm{F}\right)$

Delta T1

Delta $\mathrm{T} 2$

Log mean temperature difference $\left({ }^{\circ} \mathrm{F}\right)$

Overall heat transfer coefficient $\left(\mathrm{Btu} / \mathrm{h} / \mathrm{ft} \mathrm{t}^{2}{ }^{\circ} \mathrm{F}\right)$

Heat transfer area $\left(\mathrm{ft}^{2}\right)$

Operating pressure (psia)

Pressure factor

Materials correction factor

Module factor

(includes all of the supporting equipment and connections and installation)

Purchased cost of heat exchanger in 1987

(mild steel construction; shell and tube floating head)

CE index for process equipment in 1987

CE index for process equipment in 1995

Installed cost of heat exchanger in 1995

$59,136,171$

457

457

451

56

400

57

401

177

5

66,897

451

1.175

1

3.2

$\$ 400,000$

320

373.9

$\$ 1,757,330$

\section{Heat Exchanger for Heating Clean Fuel Gas with Raw}

\section{Gases from Gasifier}

$\mathrm{Q}=\operatorname{Load}(\mathrm{Btu} / \mathrm{h})$

Tha $=$ Inlet temperature of hot fluid $\left({ }^{\circ} \mathrm{F}\right)$

Thb $=$ Outlet temperature of hot fluid $\left({ }^{\circ} \mathrm{F}\right)$

Pressure of hot gases (psia)

$\mathrm{Tca}=$ Inlet temperature of cold fluid $\left({ }^{\circ} \mathrm{F}\right)$

$\mathrm{Tcb}=$ Outlet temperature of cold fluid $\left({ }^{\circ} \mathrm{F}\right)$

Delta T1

Delta $T 2$

Log mean temperature difference $\left({ }^{\circ} \mathrm{F}\right)$

Overall heat transfer coefficient $\left(\mathrm{Btu} / \mathrm{h} / \mathrm{ft} \mathrm{t}^{2}{ }^{\circ} \mathrm{F}\right)$

Heat transfer area $\left(\mathrm{ft}^{2}\right)$

Operating pressure (psia)

Pressure factor

Materials correction factor

Module factor

(includes all of the supporting equipment and connections and installation)

Purchased cost of heat exchanger in 1987 (mild steel construction; shell and tube floating head)

$C E$ index for process equipment in 1987

CE index for process equipment in 1995

Installed cost of heat exchanger in 1995

$344,284,466$

1,750

935

451

400

1,137

613

535

573

5

120,154

451

1.175

1

3.2

$\$ 600,770$

320

373.9 
8. Heat Exchanger for Cooling Shifted Synthesis Gas with

\section{Feedwater}

$Q=\operatorname{Load}(B t u / h)$

Tha $=$ Inlet temperature of hot fluid $\left({ }^{\circ} \mathrm{F}\right)$

$\mathrm{Thb}=$ Outlet temperature of hot fluid ( $\left.{ }^{\circ} \mathrm{F}\right)$

Pressure of hot gases (psia)

$\mathrm{Tca}=$ Inlet temperature of cold fluid $\left({ }^{\circ} \mathrm{F}\right)$

$\mathrm{Tcb}=$ Outlet temperature of cold fluid $\left({ }^{\circ} \mathrm{F}\right)$

Delta T1

Delta T2

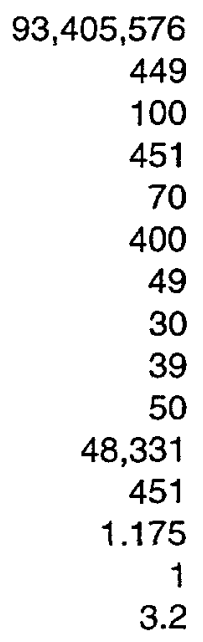

$93,405,576$

449

100

451

70

400

49

30

39

50

48,331

451

1.175

Log mean temperature difference $\left({ }^{\circ} \mathrm{F}\right)$

Overall heat transfer coefficient $\left(\mathrm{Btu} / \mathrm{h} / \mathrm{ft} 2 /{ }^{\circ} \mathrm{F}\right)$

Heat transfer area $\left(\mathrm{ft}^{2}\right)$

Operating pressure (psia)

Pressure factor

Materials correction factor

Module factor

(includes all of the supporting equipment and connections and installation)

Purchased cost of heat exchanger in 1987

(mild steel construction; shell and tube floating head)

CE index for process equipment in 1987

CE index for process equipment in 1995

Installed cost of heat exchanger in 1995 
TABLE 4.11 Sizing and Cost Estimation for Major Equipment Used for $\mathrm{CO}_{2}$ Removal in Glycol Process in Case 1

1. Gas - Gas Heat Exchanger

$Q=$ Load (Btu/h)

Tha $=$ Inlet temperature of hot fluid $\left({ }^{\circ} \mathrm{F}\right)$

$\mathrm{Thb}=$ Outlet temperature of hot fluid $\left({ }^{\circ} \mathrm{F}\right)$

Pressure of hot gases (psia)

$\mathrm{Tca}=$ Inlet temperature of cold fluid $\left({ }^{\circ} \mathrm{F}\right)$

$\mathrm{Tcb}=$ Outlet temperature of cold fluid $\left({ }^{\circ} \mathrm{F}\right)$

Delta T1

Delta T2

Log mean temperature difference $\left({ }^{\circ} \mathrm{F}\right)$

Overall heat transfer coefficient $\left(\mathrm{Btu} / \mathrm{h} / \mathrm{ft}^{2} /{ }^{\circ} \mathrm{F}\right)$

Heat transfer area $\left(\mathrm{ft}^{2}\right)$

Operating Pressure (psia)

Pressure factor

Materials correction factor

Module factor

(includes all of the supporting equipment and connections and installation)

Purchased cost of heat exchanger in 1987

(mild steel construction; shell and tube floating head)

CE index for process equipment in 1987

CE index for process equipment in 1995

$1,366,044$

70.00

55

450

30.00

56.24

13.7558

25

19

5

14,516

50

1.175

1

3.2

Installed cost of heat exchanger in 1995

$\$ 150,000$

320

373.9

$\$ 658,999$

2. $\mathrm{CO}_{2}$ Absorption Column

Diameter of tower $(\mathrm{ft})$

HETP $(\mathrm{ft})$

No. of theoretical stages

12

Absorber tower height $(\mathrm{ft})$

( $4 \mathrm{ft}$ for inlet, outlet and gas, and liquid distributors)

Volume of packing $\left(\mathrm{ft}^{3}\right)$

Pressure factor

Cost per foot of column height

(mild steel construction)

Materials correction factor

40

Module factor

4,073

$\$ 1,400$

CE index for process equipment in 1987

4.16

CE index for process equipment in 1995

320

373.9

Installed cost of absorber in 1995

Cost of packing per cubic foot

$\$ 63.5$

$\$ 272,199$

(2-in. pall rings-metal)

Total cost of packing

$\$ 258,645$ 
3. Power Recovery Turbine 1

Turbine size (hp) 649

$\begin{array}{ll}\text { Purchased cost in } 1987 & \$ 200,000\end{array}$

Module factor 1

CE index for process equipment in $1987 \quad 320$

CE index for process equipment in 1995

Installed cost of solvent pump in 1995

$\$ 233,688$

4. Slump Tank

Glycol solvent flow rate $(\mathrm{lb} / \mathrm{h}) \quad 3,308,349$

Density of glycol solvent (lb/gal) $\quad 8.6$

Residence time (s)

180

Slump tank volume (gal) $\quad 19,235$

Pressure factor $\quad 1.38$

Materials correction factor $\quad 1$

Module factor $\quad 2.08$

$\begin{array}{ll}\text { Purchased cost of slump tank in } 1987 & \$ 45,000\end{array}$

(mild steel construction)

CE index for process equipment in 1987

320

CE index for process equipment in 1995

373.9

Installed cost of slump tank in 1995

$\$ 150,925$

5. Recycle Compressor

Inlet pressure (psia)

Outlet pressure (psia)

200

446.00

Compressor size (hp)

537

Purchased cost of reciprocating compressor in 1987

$\$ 160,000$

Size factor for compressor

Materials correction factor

Module factor

CE index for process equipment in 1987

CE index for process equipment in 1995

Installed cost of compressor in 1995

$\$ 486,070$

6. Power Recovery Turbine 2

Turbine size (hp)

404

Purchased cost in 1987

$\$ 170,000$

Module factor

1

CE index for process equipment in 1987

320

CE index for process equipment in 1995

373.9

Installed cost of solvent pump in 1995

$\$ 198,634$ 
7. Flash Tank 1

Glycol flow rate $(\mathrm{lb} / \mathrm{h})$

Density of glycol (lb/gal)

$3,308,349$

Residence time (s)

Flash tank volume (gal)

Pressure factor

19,235

Module factor

Purchased cost of flash tank 1987

(mild steel construction)

$\$ 45,000$

CE index for process equipment in 1987

320

CE index for process equipment in 1995

373.9

Installed cost of flash tank in 1995

$\$ 109,366$

8. $\quad$ Flash Tank 2

Glycol flow rate $(\mathrm{lb} / \mathrm{h})$

Density of glycol (lb/gal)

Residence time (s)

Flash tank volume (gal)

Pressure factor

Module factor

Purchased cost of flash tank 1987

(mild steel construction)

CE index for process equipment in 1987

CE index for process equipment in 1995

Installed cost of flash tank in 1995

9. Flash Tank 3

Glycol flow rate (lb/h)

Density of glycol (lb/gal)

$3,308,349$

8.6

Residence time (s)

Flash tank volume (gal)

19,235

Pressure factor

Module factor

Purchased cost of flash tank 1987

(mild steel construction)

CE index for process equipment in 1987

CE index for process equipment in 1995 
TABLE 4.11 (Cont.)

10. Solvent Circulation Pump

Horsepower

Purchased cost of pump in 1987

2,205

0.79

(includes motor, coupling, base; cast iron, horizontal)

$\$ 30,000$

Materials correction factor

Module factor

1.5

320

CE index for process equipment in 1987

373.9

Installed cost of solvent pump in 1995

$\$ 254,161$

11. Compressor 1 for $\mathrm{CO}_{2}$

Inlet pressure (psia)

Outlet pressure (psia)

14.70

50.00

Compressor size (hp)

539.71

Purchased cost of reciprocating compressor in 1987

(includes electric motor drive and gear reducer)

Size factor for compressor

Materials correction factor

Module factor

160,000

CE index for process equipment in 1987

CE index for process equipment in 1995

Installed cost of compressor in 1995

373.9

12. Compressor 2 for $\mathrm{CO}_{2}$

Inlet pressure (psia)

Outlet pressure (psia)

Compressor size (hp)

Purchased cost of reciprocating compressor in 1987

(includes electric motor drive and gear reducer)

Size factor for compressor

Materials correction factor

Module factor

CE index for process equipment in 1987

CE index for process equipment in 1995

Installed cost of compressor in 1995

4.00

50.00

155.52

$\$ 60,000$

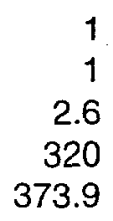

$\$ 486,070$

13. Refrigeration

Refrigeration (tons)

Purchased cost in 1987

526.71

$\$ 260,000$

Temperature correction factor

1.25

1.46

Module factor

320

CE index for process equipment in 1987

373.9

$\$ 182,276$

CE index for process equipment in 1995

Installed cost of refrigeration in 1995 
TABLE 4.11 (Cont.)

14. $\mathrm{CO}_{2}$ Product Gas Compressors

Compressor 1 (hp)

$2,582.98$

Compressor 2 (hp)

$2,582.98$

Compressor 3 (hp)

$2,582.98$

Purchased cost of centrifugal compressor 1 in 1987

$\$ 600,000$

Purchased cost of centrifugal compressor 2 in 1987

$\$ 600,000$

Purchased cost of centrifugal compressor 3 in 1987.

$\$ 600,000$

(includes electric motor drive and gear reducer)

Size factor for compressor

Module factor

CE index for process equipment in 1987

2.6

CE index for process equipment in 1995

320

Installed cost of Compressor 1 in 1995

373.9

Installed cost of Compressor 2 in 1995

Installed cost of Compressor 3 in 1995

$\$ 1,822,763$

$\$ 1,822,763$

$\$ 1,822,763$

Total Direct Cost

$\$ 9,532,478$

Total Direct Cost for Three Trains 


\section{Case 2 - Gas Turbine Topping Cycle and Membrane $\mathrm{CO}_{2}$ Recovery}

\subsection{Design Basis}

The overall system design with membrane recovery is essentially the same as that with glycol recovery as depicted in Figure 4.1, except a membrane separation unit replaces the glycol unit. The nominal $\mathrm{CO}_{2}$-removal efficiency of the membrane system is $90 \%$, although the calculated design efficiency is somewhat lower, primarily because of the methane content of the synthesis gas that remains with the hydrogen-rich retentate after separation. This methane is combusted and released as $\mathrm{CO}_{2}$ with the gas turbine exhaust. Several configurations for the membrane system were evaluated, including various series and parallel arrangements. The arrangement that most economically approaches the $90 \%$ recovery target is depicted in Figure 5.1. This system treats the sulfur-free synthesis gas flow of 11,800 pound moles per hour. The use of a recycle stream is essential to achieving the net reduction in potential $\mathrm{CO}_{2}$ emissions of $85 \%$ that is achieved with this design. In the glycol case, the absorber design assures removal of sufficient $\mathrm{CO}_{2}$ to compensate for combustion of the methane and still achieve $90 \%$ recovery. Membrane performance is not sufficient to compensate for this methane combustion. The gasifier and power island equipment are of the same scale and type as those used in the reference case and the glycol recovery case. Reduced gas turbine power output is expected because of changes in the fuel gas, but any associated changes in turbine design are not incorporated in this analysis. The substantial energy use for operation of compressors, fans, and pumps associated with gas cleanup is treated as a reduction in net output. In other words, the gross plant capacity is not increased to compensate for these losses. Table 5.1 is a summary of principal material flows for the base case and for this design option.

\subsection{Shift Reactor}

The design of the shift reactor and its integration into the system are essentially the same as those used in the glycol recovery case depicted in Section 4.2 and Figure 4.3. The key to integrating the shift reaction is to use thermal energy available from cooling the syngas to preheat the humidified fuel gas before combustion in the turbine. A slight difference in the allocation of sensible heat from initial gas cooling is evident in a comparison of Table 5.2 with Table 4.2. Specifically, less heat is allocated to the turbine fuel gas stream in the membrane case than in the glycol case, reflecting the lower temperature of the treated fuel gas after the glycol process.

\subsection{Membrane Process for $\mathrm{CO}_{2}$ Recovery}

The process flows for the glycol $\mathrm{H}_{2} \mathrm{~S}$ recovery are the same as those described in Section 4.3. Refer to that discussion for process calculations for the $\mathrm{H}_{2} \mathrm{~S}$ recovery system. In this case, the $\mathrm{H}_{2} \mathrm{~S}$-free gas is treated in the membrane system rather than by a second glycol system for $\mathrm{CO}_{2}$ recovery. The process flows for this membrane system and associated stream 


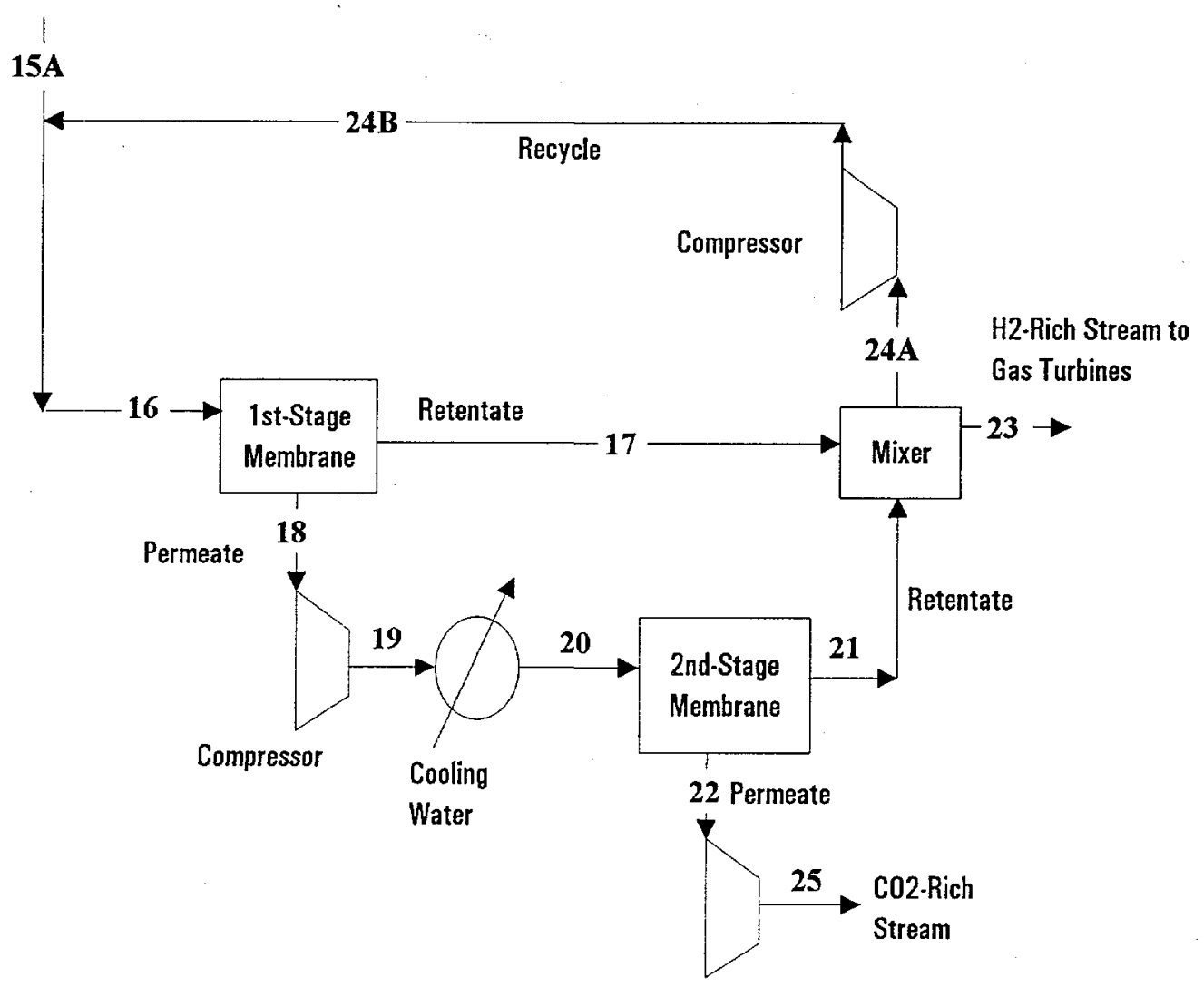

F!GURE 5.1 Flow Diagram of Membrane Process for $\mathrm{CO}_{2}$ Recovery in Case 2

TABLE 5.1 Material Flows for Oxygen-Blown Base Case and Case 2

\begin{tabular}{|c|c|c|}
\hline Material Flow (tons/d) & Base Case & Case 2 \\
\hline Coal (prepared) & 3,845 & 3,845 \\
\hline Limestone & 0 & 0 \\
\hline Air & 0 & 0 \\
\hline Oxygen & 2,347 & 2,347 \\
\hline Solid waste & 492 & 492 \\
\hline Sulfur & 78 & 78 \\
\hline $\mathrm{CO}_{2}$ (gasifier only) & 8,586 & 1,227 \\
\hline $\mathrm{SO}_{2}$ (gasifier only) & 6.92 & 6.92 \\
\hline Net power output (MW) & 413.5 & 330 \\
\hline
\end{tabular}


TABLE 5.2 Heat Recovery and Allocation ( $10^{6} \mathrm{Btu} / \mathrm{h}$ ) for Gas Turbine/Membrane Process in Case 2

\begin{tabular}{lcccc}
\hline \multicolumn{1}{c}{ Process } & $\begin{array}{c}\text { Enthalpy Change } \\
\text { Available from } \\
\text { Process }\end{array}$ & $\begin{array}{c}\text { Allocation to } \\
\text { Fuel Gas } \\
\text { Preheating }\end{array}$ & $\begin{array}{c}\text { Allocation for } \\
\text { Raising Steam for } \\
\text { Shift System }\end{array}$ & $\begin{array}{c}\text { Allocation } \\
\text { to Steam } \\
\text { Cycle }\end{array}$ \\
\hline Initial gas cooling to $460^{\circ} \mathrm{F}$ & 513.89 & 327.22 & 123.89 & 62.78 \\
Cooling after first-stage shift & 168.21 & 0.00 & 168.21 & 0.00 \\
Cooling after second-stage shift & 673.27 & 171.84 & 215.65 & 285.78 \\
\hline
\end{tabular}

calculations are summarized in Tables 5.3 and 5.4, respectively. The high level of recycle is needed to achieve the $\mathrm{CO}_{2}$ recovery goal. The membrane technology selected for this study is the facilitated transport membrane, which incorporates an absorbent fluid layer held between two films. Such a membrane can have a high selectivity for $\mathrm{H}_{2} / \mathrm{CO}_{2}$ separation, although low permeability results in high cost. A more conventional membrane of single-layer polymeric or metallic material that is capable of effectively separating $\mathrm{CO}_{2}$ from $\mathrm{H}_{2}$ is not available. One scheme that has been proposed to circumvent this problem (Hendriks 1994) applies such conventional membranes directly to the synthesis gas without shift. The problem then is separation of $\mathrm{CO}$ from $\mathrm{H}_{2}$.

The resulting $\mathrm{CO}$-rich and $\mathrm{H}_{2}$-rich streams are then used to fuel separate gas turbines. The exhaust from the $\mathrm{CO}$ turbine is a fairly pure $\mathrm{CO}_{2}$ stream if oxygen is used as oxidant. The tradeoff is largely in the extra cost of air separation versus that of the more expensive membrane evaluated in this study.

\subsection{Gas Turbine, Steam Cycle, and Plant Performance}

A summary of power generation and internal power consumption when the membrane system is used for $\mathrm{CO}_{2}$ recovery is presented in Table 5.5. The energy consumed by the $\mathrm{CO}_{2}$-recovery system and the loss in gas turbine output, which is primarily a result of lost methane, result in an energy penalty of $20 \%$ relative to the base case generation. This result is compared in Table 5.6 with the glycol-based recovery system, which imposes an energy penalty of $9 \%$ relative to the base case.

\subsection{Economics}

Details of the capital investment estimates for the $\mathrm{H}_{2} \mathrm{~S}$ recovery system, the shift system, and the $\mathrm{CO}_{2}$ recovery system are presented in Tables 5.7, 5.8, and 5.9, respectively. 
TABLE 5.3 Stream Flows of Membrane Process for $\mathrm{CO}_{2}$ Removal in Case 2

\begin{tabular}{|c|c|c|c|c|c|c|}
\hline Stream Data & Stream 15A & Stream 16 & Stream 17 & Stream 18 & Stream 19 & Stream 20 \\
\hline Description of stream & $\begin{array}{l}\text { Sulfur-free feed gas } \\
\text { from } \mathrm{H}_{2} \mathrm{~S} \text { removal } \\
\text { section }\end{array}$ & $\begin{array}{l}\text { Feed to 1st-stage } \\
\text { membrane }\end{array}$ & $\begin{array}{l}\text { Retentate from 1st- } \\
\text { stage membrane }\end{array}$ & $\begin{array}{l}\text { Permeate from } \\
\text { 1st-stage } \\
\text { membrane }\end{array}$ & $\begin{array}{l}\text { Permeate oí ist } \\
\text { stage after } \\
\text { compressor }\end{array}$ & $\begin{array}{l}\text { Permeate of ist } \\
\text { stage after heat } \\
\text { exchange }\end{array}$ \\
\hline \multicolumn{7}{|l|}{ Gases $(l b \cdot \mathrm{mol} / \mathrm{h})$} \\
\hline $\mathrm{CO}$ & 45.13 & 70.47 & 24.80 & 45.67 & 45.67 & 45.67 \\
\hline $\mathrm{CO}_{2}$ & $4,310.02$ & $5,007.88$ & 598.56 & $4,409.32$ & $4,409.32$ & $4,409.32$ \\
\hline $\mathrm{H}_{2}$ & $6,822.47$ & $17,840.18$ & $16,725.17$ & $1,115.01$ & $1,115.01$ & $1,115.01$ \\
\hline $\mathrm{H}_{2} \mathrm{O}$ & 0.00 & 0.00 & 0.00 & 0.00 & 0.00 & 0.00 \\
\hline $\mathrm{N}_{2}$ & 35.71 & 81.47 & 56.50 & 24.97 & 24.97 & 24.97 \\
\hline $\mathrm{Ar}$ & 72.72 & 114.34 & 40.87 & 73.47 & 73.47 & 73.47 \\
\hline $\mathrm{CH}_{4}$ & 463.02 & 941.95 & 542.39 & 399.56 & 399.56 & 399.56 \\
\hline $\mathrm{NH}_{3}$ & 18.79 & 19.42 & 0.51 & 18.91 & 18.91 & 18.91 \\
\hline $\mathrm{H}_{2} \mathrm{~S}$ & 0.63 & 0.65 & 0.02 & 0.64 & 0.64 & 0.64 \\
\hline $\mathrm{HCl}$ & 0.41 & 0.43 & 0.01 & 0.41 & 0.41 & 0.41 \\
\hline $\mathrm{O}_{2}$ & 0.00 & 0.00 & 0.00 & 0.00 & 0.00 & 0.00 \\
\hline $\cos$ & 2.97 & 3.07 & 0.08 & 2.99 & 2.99 & 2.99 \\
\hline $\mathrm{SO}_{2}$ & 0.00 & 0.00 & 0.00 & 0.00 & 0.00 & 0.00 \\
\hline Total gas flow & $11,771.88$ & $24,079.86$ & $17,988.91$ & $6,090.95$ & $6,090.95$ & $6,090.95$ \\
\hline Liquids (lb.mol/h) & 0.00 & 0.00 & 0.00 & 0.00 & 0.00 & 0.00 \\
\hline Temperature $\left({ }^{\circ} \mathrm{F}\right)$ & 70 & 86.62 & 86.62 & 86.62 & 538.71 & 212.00 \\
\hline Pressure (psia) & 445 & 445.00 & 435.00 & 45.00 & 445.00 & 445.00 \\
\hline $\begin{array}{l}\text { Enthalpy of stream }(\mathrm{Btu} / \mathrm{h}) \\
\left(\text { reference, } 32^{\circ} \mathrm{F}\right)\end{array}$ & $3,450,035$ & $9,738,040$ & $6,911,024$ & $2,827,016$ & $28,819,717$ & $9,580,971$ \\
\hline
\end{tabular}


TABLE 5.3 (Cont.)

\begin{tabular}{|c|c|c|c|c|c|}
\hline Stream Data & Stream 21 & Stream 22 & Stream 23 & Stream 24A & Stream 24B \\
\hline Description of stream & $\begin{array}{l}\text { Retentate from } 2 \text { nd- } \\
\text { stage membrane }\end{array}$ & $\begin{array}{l}\text { Permeate from 2nd- } \\
\text { stage membrane }\end{array}$ & $\begin{array}{l}\text { Fuel gas to gas } \\
\text { turbines }\end{array}$ & $\begin{array}{l}\text { Recycle to 1st- } \\
\text { stage membrane }\end{array}$ & $\begin{array}{l}\text { Compressed recycle to } \\
\text { 1st-stage membrane }\end{array}$ \\
\hline \multicolumn{6}{|l|}{ Gases $(\mathrm{lb} \cdot \mathrm{mol} / \mathrm{h})$} \\
\hline $\mathrm{CO}$ & 16.07 & 29.60 & 15.53 & 25.34 & 25.34 \\
\hline $\mathrm{CO}_{2}$ & 527.01 & $3,882.31$ & 427.72 & 697.85 & 697.85 \\
\hline $\mathrm{H}_{2}$ & $1,045.32$ & 69.69 & $6,752.79$ & $11,017.70$ & $11,017.70$ \\
\hline $\mathrm{H}_{2} \mathrm{O}$ & 0.00 & 0.00 & 0.00 & 0.00 & 0.00 \\
\hline $\mathrm{N}_{2}$ & 17.32 & 7.65 & 28.05 & 45.77 & 45.77 \\
\hline $\mathrm{Ar}$ & 26.26 & 47.21 & 25.51 & 41.62 & 41.62 \\
\hline $\mathrm{CH}_{4}$ & 230.07 & 169.49 & 293.54 & 478.93 & 478.93 \\
\hline $\mathrm{NH}_{3}$ & 0.50 & 18.41 & 0.38 & 0.63 & 0.63 \\
\hline $\mathrm{H}_{2} \mathrm{~S}$ & 0.02 & 0.62 & 0.01 & 0.02 & 0.02 \\
\hline $\mathrm{HCl}$ & 0.01 & 0.40 & 0.01 & 0.01 & 0.01 \\
\hline $\mathrm{O}_{2}$ & 0.00 & 0.00 & 0.00 & 0.00 & 0.00 \\
\hline $\cos$ & 0.08 & 2.91 & 0.06 & 0.10 & 0.10 \\
\hline $\mathrm{SO}_{2}$ & 0.00 & 0.00 & 0.00 & 0.00 & 0.00 \\
\hline Total gas flow & $1,862.67$ & $4,228.28$ & $7,543.60$ & $12,307.98$ & $12,307.98$ \\
\hline Liquids $(\mathrm{lb} \cdot \mathrm{mol} / \mathrm{h})$ & 0.00 & 0.00 & 0.00 & 0.00 & 0.00 \\
\hline Temperature $\left({ }^{\circ} \mathrm{F}\right)$ & 212.00 & 212.00 & 99.64 & 99.64 & 103.98 \\
\hline Pressure (psia) & 435.00 & 45.00 & 435.00 & 435.00 & 445.00 \\
\hline $\begin{array}{l}\text { Enthalpy of stream }(B t u / h) \\
\left.\text { (reference, } 32^{\circ} \mathrm{F}\right)\end{array}$ & $2,617,896$ & $6,963,075$ & $3,620,990$ & $5,907,930$ & $6,288,005$ \\
\hline
\end{tabular}


TABLE 5.4 Descriptions of Streams of Membrane Process for $\mathrm{CO}_{2}$ Removal in Case 2

\begin{tabular}{ccc}
\hline Stream and & & \\
Characteristics & Data & Comments on Stream Calculations \\
\hline
\end{tabular}

Stream 15A: Sulfur-free gas from

$\mathrm{H}_{2} \mathrm{~S}$ section

Temperature $\left({ }^{\circ} \mathrm{F}\right)$

Pressure (psia)

Flow rate $(\mathrm{lb} \cdot \mathrm{mol} / \mathrm{h})$

$\mathrm{CO}_{2}$ (mole fraction)

$\mathrm{H}_{2} \mathrm{~S}$ (mole fraction)

Stream 16: Feed gas to 1st-stage membrane system

Temperature $\left({ }^{\circ} \mathrm{F}\right)$

Pressure (psia)

Flow rate $(\mathrm{lb} \cdot \mathrm{mol} / \mathrm{h})$

$\mathrm{CO}_{2}$ (mole fraction)

$\mathrm{H}_{2} \mathrm{~S}$ (mole fraction)

Stream 17: Retentate from 1st-stage membrane system

Temperature $\left({ }^{\circ} \mathrm{F}\right)$

Pressure (psia)

Flow rate $(\mathrm{lb} \cdot \mathrm{mol} / \mathrm{h})$

$\mathrm{CO}_{2}$ (mole fraction)

$\mathrm{H}_{2} \mathrm{~S}$ (mole fraction)

Stream 18: Permeate from 1st-stage membrane system

Temperature $\left({ }^{\circ} \mathrm{F}\right)$

Pressure (psia)

Flow rate $(\mathrm{lb} \cdot \mathrm{mol} / \mathrm{h})$

$\mathrm{CO}_{2}$ (mole fraction)

$\mathrm{H}_{2} \mathrm{~S}$ (mole fraction)

Stream 19: Gases from compressor

Temperature $\left({ }^{\circ} \mathrm{F}\right)$

Pressure (psia)

Flow rate $(\mathrm{lb} \cdot \mathrm{mol} / \mathrm{h})$

$\mathrm{CO}_{2}$ (mole fraction)

$\mathrm{H}_{2} \mathrm{~S}$ (mole fraction)
70

445

$11,771.88$

0.3661

0.0001

86.62

445

$24,079.86$

0.2080

0.0000

86.62

435

$17,988.91$

0.0333

0.0000

86.62 The composition of this stream is

45 calculated by mass balance around the

$6,090.95$ membrane.

0.7329

0.0001

538

445

$6,090.95$

0.7329

0.0001

The synthesis gas is cleaned in two stages. First sulfur compounds are removed. Then they are fed to the membrane system for $\mathrm{CO}_{2}$ recovery.

The sulfur-free gas is mixed with the recycle from the 2 nd-stage retentate and fed to the 1st-stage membranes.

The composition of this stream depends on the permeability and selectivity of the membranes. The membrane system is a facilitated membrane that has a higher selectivity and permeability for $\mathrm{CO}_{2}$ than $\mathrm{H}_{2}$.

$$
\text { membrane. }
$$

The permeate from 1st-stage membrane systems is at a pressure of $45 \mathrm{psia}$. These gases are again compressed to a pressure of 445 psia for the 2nd-stage membrane system. 
TABLE 5.4 (Cont.)

\begin{tabular}{|c|c|c|}
\hline $\begin{array}{l}\text { Stream and } \\
\text { Characteristics }\end{array}$ & Data & Comments on Stream Calculations \\
\hline \multicolumn{3}{|c|}{ Stream 20: Gases from heat exchanger } \\
\hline Temperature $\left({ }^{\circ} \mathrm{F}\right)$ & 212 & \multirow{5}{*}{$\begin{array}{l}\text { The temperature of the gases rises because of } \\
\text { the compression. Therefore, this stream is } \\
\text { cooled to a temperature of } 212^{\circ} \mathrm{F} \text {, suitable } \\
\text { for the membrane system. }\end{array}$} \\
\hline Pressure (psia) & 445 & \\
\hline Flow rate $(\mathrm{lb} \cdot \mathrm{mol} / \mathrm{h})$ & $6,090.95$ & \\
\hline $\mathrm{CO}_{2}$ (mole fraction) & 0.7329 & \\
\hline $\mathrm{H}_{2} \mathrm{~S}$ (mole fraction) & 0.0001 & \\
\hline \multicolumn{3}{|c|}{$\begin{array}{l}\text { Stream 21: Retentate of 2nd-stage } \\
\text { membrane system }\end{array}$} \\
\hline Temperature $\left({ }^{\circ} \mathrm{F}\right)$ & 212 & \multirow{5}{*}{$\begin{array}{l}\text { The composition of this stream is calculated } \\
\text { on the basis of the selectivity and permeability of } \\
\text { gases, as is done for stream } 17 \text {. }\end{array}$} \\
\hline Pressure (psia) & 435 & \\
\hline Flow rate $(\mathrm{lb} \cdot \mathrm{mol} / \mathrm{h})$ & $1,862.67$ & \\
\hline $\mathrm{CO}_{2}$ (mole fraction) & 0.2829 & \\
\hline $\mathrm{H}_{2} \mathrm{~S}$ (mole fraction) & 0.0000 & \\
\hline \multicolumn{3}{|c|}{$\begin{array}{l}\text { Stream 22: Permeate of } 2 \text { nd-stage } \\
\text { membrane system }\end{array}$} \\
\hline Temperature $\left({ }^{\circ} \mathrm{F}\right)$ & 212 & \multirow{5}{*}{$\begin{array}{l}\text { The composition of this stream is } \\
\text { calculated on the basis of the mass balance } \\
\text { around the membrane. This is the rich- } \mathrm{CO}_{2} \\
\text { stream for disposal. }\end{array}$} \\
\hline Pressure (psia) & 45 & \\
\hline Flow rate $(\mathrm{lb} \cdot \mathrm{mol} / \mathrm{h})$ & $4,228.28$ & \\
\hline $\mathrm{CO}_{2}$ (mole fraction) & 0.9182 & \\
\hline $\mathrm{H}_{2} \mathrm{~S}$ (mole fraction) & 0.0001 & \\
\hline \multicolumn{3}{|c|}{ Stream 23: Fuel gas to gas turbines } \\
\hline Temperature $\left({ }^{\circ} \mathrm{F}\right)$ & 99.64 & \multirow{5}{*}{$\begin{array}{l}\mathrm{H}_{2} \text {-rich retentate from the 1st stage (stream 17) } \\
\text { and that from the } 2 \text { nd stage (stream } 21 \text { ) are } \\
\text { mixed, and part of mixture is taken as fuel } \\
\text { gas for gas turbines. }\end{array}$} \\
\hline Pressure (psia) & 435 & \\
\hline Flow rate $(\mathrm{lb} \cdot \mathrm{mol} / \mathrm{h})$ & $7,543.60$ & \\
\hline $\mathrm{CO}_{2}$ (mole fraction) & 0.0567 & \\
\hline $\mathrm{H}_{2} \mathrm{~S}$ (mole fraction) & 0.0000 & \\
\hline \multicolumn{3}{|c|}{$\begin{array}{l}\text { Stream 24A: Recycle to 1st-stage } \\
\text { membrane system }\end{array}$} \\
\hline Temperature $\left({ }^{\circ} \mathrm{F}\right)$ & 99.64 & \multirow{5}{*}{$\begin{array}{l}\text { Part of the retentate from stream } 17 \text { and part } \\
\text { from stream } 21 \text { are recycled back to the } \\
1 \text { st-stage membrane systems to increase the } \\
\mathrm{CO}_{2} \text {-removal efficiency. }\end{array}$} \\
\hline Pressure (psia) & 435 & \\
\hline Flow rate $(\mathrm{lb} \cdot \mathrm{mol} / \mathrm{h})$ & $12,307.98$ & \\
\hline $\mathrm{CO}_{2}$ (mole fraction) & 0.0567 & \\
\hline $\mathrm{H}_{2} \mathrm{~S}$ (mole fraction) & 0.0000 & \\
\hline \multicolumn{3}{|c|}{$\begin{array}{l}\text { Stream 24B: Recycle to } 1 \text { st-stage } \\
\text { membrane after compression }\end{array}$} \\
\hline Temperature $\left({ }^{\circ} \mathrm{F}\right)$ & 103.98 & \multirow{5}{*}{$\begin{array}{l}\text { The recycle from the retentate is at a } \\
\text { pressure of } 435 \text { psia and is compressed to } \\
\text { the inlet pressure of the } 1 \mathrm{st} \text { membrane. }\end{array}$} \\
\hline Pressure (psia) & 445 & \\
\hline Flow rate $(\mathrm{lb} \cdot \mathrm{mol} / \mathrm{h})$ & $12,307.98$ & \\
\hline $\mathrm{CO}_{2}$ (mole fraction) & 0.0567 & \\
\hline $\mathrm{H}_{2} \mathrm{~S}$ (mole fraction) & 0.0000 & \\
\hline
\end{tabular}


TABLE 5.5 Turbine Output, Plant Power Use, and Net Power Output for Base Case and Case 2 Gas Turbine/Membrane Process

\begin{tabular}{lcc}
\hline & \multicolumn{2}{c}{ Power (MW) } \\
\cline { 2 - 3 } Power Variable & Base Case & Membrane Case \\
\hline Power output & & \\
Gas turbine & 298.8 & 262.8 \\
Steam turbine & 159.4 & 154.8 \\
& & \\
Internal power consumption & & \\
CO $_{2}$ recovery & 0.0 & -20.0 \\
CO ${ }_{2}$ compression & 0.0 & -0.9 \\
Solvent circulation & 0.0 & -3.0 \\
Solvent refrigeration & 0.0 & -19.0 \\
Others & -44.7 & -44.7 \\
Gasification system & & \\
Net power output & 413.5 & 330.0 \\
Energy penalty & 0.0 & 83.5 \\
\hline
\end{tabular}

TABLE 5.6 Overall Power Recovery and Production for Three Gas Turbine Cases

\begin{tabular}{lccc}
\hline & \multicolumn{3}{c}{ Power (MW) } \\
\cline { 2 - 4 } \multicolumn{1}{c}{ Power Variable } & Base Case & $\begin{array}{c}\text { Glycol } \\
\text { Case 1 }\end{array}$ & $\begin{array}{c}\text { Membrane } \\
\text { Case 2 }\end{array}$ \\
\hline $\begin{array}{l}\text { Power output } \\
\text { Gas turbine }\end{array}$ & & \\
$\quad$ Steam turbine & 298.8 & 284.8 & 262.8 \\
Internal power consumption & 159.4 & 161.6 & 154.8 \\
$\quad \begin{array}{l}\text { CO }{ }_{2} \text { recovery } \\
\text { Gasification system }\end{array}$ & 0.0 & -24.2 & -42.9 \\
Net power output & -44.7 & -44.7 & -44.7 \\
Energy penalty & 413.5 & 377.5 & 330.0 \\
\hline
\end{tabular}


TABLE 5.7 Sizing and Cost Estimation for Major Equipment Used for $\mathrm{H}_{2} \mathrm{~S}$ Removal in Glycol Process in Case 2

1. Heat Exchanger before the Absorption Column

$Q=\operatorname{Load}(B t u / h)$

Tha $=$ Inlet temperature of hot fluid $\left({ }^{\circ} \mathrm{F}\right)$

$3,630,585$

100

$\mathrm{Thb}=$ Outlet temperature of hot fluid $\left({ }^{\circ} \mathrm{F}\right)$

63

Pressure of hot gases (psia)

451

$\mathrm{Tca}=$ Inlet temperature of cold fluid $\left({ }^{\circ} \mathrm{F}\right)$

30

$\mathrm{Tcb}=$ Outlet temperature of cold fluid $\left({ }^{\circ} \mathrm{F}\right)$

Delta T1

Delta T2

70

30

33

Log mean temperature difference $\left({ }^{\circ} \mathrm{F}\right)$

Overall heat transfer coefficient $\left(\mathrm{Btu} / \mathrm{h} / \mathrm{ft}^{2} /{ }^{\circ} \mathrm{F}\right)$

Heat transfer area $\left(\mathrm{ft}^{2}\right)$

Operating pressure (psia)

5

23,070

451

Pressure factor

1.175

Materials correction factor

Module factor

(includes all of the supporting equipment and connections and installation)

Purchased cost of heat exchanger in 1987

(mild steel construction; shell and tube floating head)

CE index for process equipment in 1987

CE index for process equipment in 1995

3.2

Installed cost of heat exchanger in 1995

$\$ 185,000$

320

373.9

$\$ 812,765$

2. $\mathrm{H}_{2} \mathrm{~S}$ Absorption Column

Diameter of tower $(\mathrm{ft})$

HETP $(\mathrm{ft})$

No. of theoretical stages

Absorber tower height ( $\mathrm{ft}$ )

(4 ft for inlet, outlet and gas, and liquid distributors)

Volume of packing $\left(\mathrm{ft}^{3}\right)$

Pressure factor

Cost per foot of column height per foot

(mild steel construction)

Materials correction factor

Module factor

CE index for process equipment in 1987

CE index for process equipment in 1995

Installed cost of absorber in 1995

Cost of packing per cubic foot

(2-in. pall rings-metal)

Total cost of packing

$\$ 505,513$

$\$ 63.5$

1,810

2.6

$\$ 1,000$

4.16

320

373.9
8

12

40

1

0

.


3. Power Recovery Turbine 1

Turbine size (hp)

173

Purchased cost in 1987

$\$ 120,000$

Module factor

CE index for process equipment in 1987

CE index for process equipment in 1995

373.9

Installed cost of solvent pump in 1995

$\$ 175,266$

4. Slump Tank

Glycol solvent flow rate $(\mathrm{lb} / \mathrm{h})$

Density of glycol solvent (lb/gal)

613,374

8.6

180

Residence time (s)

3,566

Pressure factor

Materials correction factor

1

1

Module factor

2.08

Purchased cost of slump tank in 1987

$\$ 13,000$

(mild steel construction)

CE index for process equipment in 1987

320

$\mathrm{CE}$ index for process equipment in 1995

373.9

Installed cost of slump tank in 1995

$\$ 31,595$

5. Power Recovery Turbine 2

Turbine size (hp)

Purchased cost in 1987

CE index for process equipment in 1987

373.9

Installed cost of solvent pump in 1995

$\$ 75,948$

6. Solvent Circulation Pump

Horsepower

403

Purchased cost of pump in 1987

$\$ 30,000$

(includes motor, coupling, base; cast iron, horizontal)

Materials correction factor

Module factor

CE index for process equipment in 1987

CE index for process equipment in 1995

373.9

Installed cost of solvent pump in 1995

$\$ 52,580$ 
7. Lean-Rich Solvent Heat Exchanger

$Q=$ Load (Btu/h)

Tha $=$ Inlet temperature of hot fluid $\left({ }^{\circ} \mathrm{F}\right)$

$\mathrm{Thb}=$ Outlet temperature of hot fluid $\left({ }^{\circ} \mathrm{F}\right)$

Pressure of hot gases (psia)

Tca $=$ Inlet temperature of cold fluid $\left({ }^{\circ} \mathrm{F}\right)$

$\mathrm{Tcb}=$ Outlet temperature of cold fluid $\left({ }^{\circ} \mathrm{F}\right)$

Delta $T 1$

Delta T2

$47,524,550$

215.21

67

450

42.10

190.00

25.2077

Log mean temperature difference $\left({ }^{\circ} \mathrm{F}\right)$

25

25

150

Overall heat transfer coefficient (Btu/h/ft $/{ }^{\circ} \mathrm{F}$ )

Heat transfer area $\left(\mathrm{ft}^{2}\right)$

12,697

Operating pressure (psia)

50

Pressure factor

Materials correction factor

Module factor

(includes all of the supporting equipment and connections and installation)

Purchased cost of heat exchanger in 1987

(mild steel construction; shell and tube floating head)

CE index for process equipment in 1987

CE index for process equipment in 1995

Installed cost of heat exchanger in 1995

$\$ 448,680$

8. Stripping Column

Diameter of tower (ft)

HETP (ft)

No. of theoretical stages

Absorber tower height

( $4 \mathrm{ft}$ for inlet, outlet and gas, and liquid distributors)

Volume of packing $\left(\mathrm{ft}^{3}\right)$

Pressure factor

Materials correction factor (stainless steel 304)

Cost per ft of column height

(mild steel construction)

Module factor

$\$ 120,000$

320

373.9

CE index for process equipment in 1987

$C E$ index for process equipment in 1995

Installed cost of absorber in 1995

Cost of packing per cubic foot

(2-in. pall rings-SS)

Materials correction factor

Total cost of packing

$\$ 396,633$

$\$ 63.5$

4.16

320

373.9

1

$\$ 179,614$ 
9. Overhead Condenser

$\mathrm{Q}=\operatorname{Load}(\mathrm{Btu} / \mathrm{h})$

Tha $=$ Inlet temperature of hot fluid $\left({ }^{\circ} \mathrm{F}\right)$

$\mathrm{Thb}=$ Outlet temperature of hot fluid $\left({ }^{\circ} \mathrm{F}\right)$.

Pressure of hot gases (psia)

$\mathrm{Tca}=$ Inlet temperature of cold fluid $\left({ }^{\circ} \mathrm{F}\right)$

$\mathrm{Tcb}=$ Outlet temperature of cold fluid $\left({ }^{\circ} \mathrm{F}\right)$

Delta T1

Delta $T 2$

Log mean temperature difference $\left({ }^{\circ} \mathrm{F}\right)$

Overall heat transfer coefficient (Btu/h/ft ${ }^{2} /{ }^{\circ} \mathrm{F}$ )

Heat transfer area $\left(\mathrm{ft}^{2}\right)$

Operating Pressure (psia)

Pressure factor

Materials correction factor 1 for SS

Materials correction factor 2 for SS

Materials correction factor

Module factor

(includes all of the supporting equipment and connections and installation)

Purchased cost of heat exchanger in 1987

(mild steel construction; shell and tube floating Head)

CE index for process equipment in 1987

CE index for process equipment in 1995

$20,323,399$

212.00

100

14.7

70.00

180.00

32

30

31

40

16,396

14.7

1

2.7

0.07

3.48

3.2

Installed cost of heat exchanger in 1995

$\$ 160,000$

320

373.9

$\$ 2,079,839$

10. Phase Separator

Flow rate $(\mathrm{lb} / \mathrm{h})$

22,291

0.04

Density of fluid (lb/gal)

120

Residence time (s)

18,576

Pressure factor

1

Materials correction factor (stainless steel)

1.8

Module factor

2.08

Purchased cost of phase separator in 1987

(mild steel construction)

CE index for process equipment in 1987

$\$ 44,000$

CE index for process equipment in 1995

320

373.9

$\$ 192,484$ 
TABLE 5.7 (Cont.)

11. Solvent Refrigeration

Refrigeration (tons)

Purchased cost in 1987

Temperature correction factor

Module factor

4001.06

CE index for process equipment in 1987

CE index for process equipment in 1995

Installed cost of refrigeration in 1995

$\$ 852,959$

Total Direct Cost

$\$ 5,918,829$

Total Direct Cost for Three Trains 
TABLE 5.8 Sizing and Cost Estimation for Major Equipment Used for Shift System in Case 2

1. First-Stage Shift Reactor

Catalyst volume $\left(\mathrm{ft}^{3}\right)$

665

Reactor volume $\left(\mathrm{ft}^{3}\right)$ (1.2 times the catalyst volume)

798

Reactor volume (gal)

5,969

Pressure factor

Module factor

3.05

CE index for process equipment in 1987

320

CE index for process equipment in 1995

373.9

Purchased cost of reactor in 1987

$\$ 7,000$

Installed cost of reactor in 1995

$\$ 69,849$

2. Second-Stage Shift Reactor

Catalyst volume $\left(\mathrm{ft}^{3}\right)$

Reactor volume $\left(\mathrm{ft}^{3}\right)$ (1.2 times the catalyst volume)

Reactor volume (gal)

2,558

Pressure factor

2.8

Module factor

3.05

CE index for process equipment in 1987

320

CE index for process equipment in 1995

373.9

Purchased cost of reactor in 1987

$\$ 5,000$

Installed cost of reactor in 1995

$\$ 49,849$

3. Cost of Shift Catalyst

Volume of catalyst in first stage $\left(\mathrm{ft}^{3}\right)$

Volume of catalyst in second stage $\left(\mathrm{ft}^{3}\right)$

339

Cost of high-temperature catalyst per cubic foot

$\$ 50$

Cost of low-temperature catalyst per cubic foot

$\$ 250$

Total cost of catalyst 
4. Heat Exchanger between First- and Second-Shift Stages

$Q=$ Load (Btu/h)

Tha $=$ Inlet temperature of hot fluid $\left({ }^{\circ} \mathrm{F}\right)$

Thb $=$ Outlet temperature of hot fluid $\left({ }^{\circ} \mathrm{F}\right)$

Pressure of hot gases (psia)

$\mathrm{Tca}=$ Inlet temperature of cold fluid $\left({ }^{\circ} \mathrm{F}\right)$

$\mathrm{Tcb}=$ Outlet temperature of cold fluid $\left({ }^{\circ} \mathrm{F}\right)$

Delta T1

Delta T2

Log mean temperature difference $\left({ }^{\circ} \mathrm{F}\right)$

Overall heat transfer coefficient $\left(\mathrm{Btu} / \mathrm{h} / \mathrm{ft}^{2} /{ }^{\circ} \mathrm{F}\right)$

Heat transfer area $\left(\mathrm{ft}^{2}\right)$

Operating pressure (psia)

$56,070,250$

684

457

451

400

457

226

57

123

40

11,383

451

Pressure factor

1.175

Materials correction factor

Module factor

(includes all of the supporting equipment and connections and installation)

Purchased cost of heat exchanger in 1987

(mild steel construction; shell and tube floating head)

CE index for process equipment in 1987

CE index for process equipment in 1995

Installed cost of heat exchanger in 1995

$\$ 527,199$

5. Heat Exchanger after Second-Stage Shift for Raising Steam

$\mathrm{Q}=$ Load (Btu/h)

Tha $=$ Inlet temperature of hot fluid $\left({ }^{\circ} \mathrm{F}\right)$

$\mathrm{Thb}=$ Outlet temperature of hot fluid $\left({ }^{\circ} \mathrm{F}\right)$

Pressure of hot gases (psia)

Tca $=$ Inlet temperature of cold fluid $\left({ }^{\circ} \mathrm{F}\right)$

$\mathrm{Tcb}=$ Outlet temperature of cold fluid $\left({ }^{\circ} \mathrm{F}\right)$

Delta $T 1$

Delta T2

Log mean temperature difference $\left({ }^{\circ} \mathrm{F}\right)$

Overall heat transfer coefficient (Btu/h/ft $2 /{ }^{\circ} \mathrm{F}$ )

Heat transfer area $\left(\mathrm{ft}^{2}\right)$

Operating pressure (psia)

Pressure factor

Materials correction factor

Module factor

(includes all of the supporting equipment and connections and installation)

Purchased cost of heat exchanger in 1987

(mild steel construction; shell and tube floating head)

CE index for process equipment in 1987

$71,881,771$

457

457

451

100

400

57

357

164

40

10,955

451

1.175

1

3.2

CE index for process equipment in 1995

Installed cost of heat exchanger in 1995 
TABLE 5.8 (Cont.)

6. Heat Exchanger after Second-Stage for Heating Fuel Gas

$\mathrm{Q}=$ Load (Btu/h)

Tha $=$ Inlet temperature of hot fluid $\left({ }^{\circ} \mathrm{F}\right)$

$57,280,972$

457

457

$\mathrm{Thb}=$ Outlet temperature of hot fluid $\left({ }^{\circ} \mathrm{F}\right)$

451

Pressure of hot gases (psia)

100

Tca $=$ Inlet temperature of cold fluid $\left({ }^{\circ} \mathrm{F}\right)$

400

$\mathrm{Tcb}=$ Outlet temperature of cold fluid ( $\left.{ }^{\circ} \mathrm{F}\right)$

57

Delta T1

Delta T2

356

Log mean temperature difference $\left({ }^{\circ} \mathrm{F}\right)$

164

Overall heat transfer coefficient $\left(\mathrm{Btu} / \mathrm{h} / \mathrm{ft}^{2} /{ }^{\circ} \mathrm{F}\right)$

Heat transfer area $\left(\mathrm{ft}^{2}\right)$

Operating pressure (psia)

70,015

451

Pressure factor

1.175

Materials correction factor

Module factor

(includes all of the supporting equipment and connections and installation)

Purchased cost of heat exchanger in 1987

(mild steel construction; shell and tube floating head)

CE index for process equipment in 1987

CE index for process equipment in 1995

3.2

Installed cost of heat exchanger in 1995

$\$ 400,000$

320

373.9

$\$ 1,757,330$

7. Heat Exchanger for Heating Clean Fuel Gas with Raw

Gases from Gasifier

$Q=\operatorname{Load}(B t u / h)$

Tha $=$ Inlet temperature of hot fluid $\left({ }^{\circ} \mathrm{F}\right)$

Thb $=$ Outlet temperature of hot fluid $\left({ }^{\circ} \mathrm{F}\right)$

Pressure of hot gases (psia)

$\mathrm{Tca}=$ Inlet temperature of cold fluid $\left({ }^{\circ} \mathrm{F}\right)$

$\mathrm{Tcb}=$ Outlet temperature of cold fluid $\left({ }^{\circ} \mathrm{F}\right)$

Delta $T 1$

Delta T2

Log mean temperature difference $\left({ }^{\circ} \mathrm{F}\right)$

Overall heat transfer coefficient $\left(\mathrm{Btu} / \mathrm{h} / \mathrm{ft} 2 /{ }^{\circ} \mathrm{F}\right)$

$327,214,827$

1,750

977

465

400

1,100

650

577

613

5

106,782

465

Operating pressure (psia)

1.175

Materials correction factor

Module factor

(includes all of the supporting equipment and connections and installation)

Purchased cost of heat exchanger in 1987

(mild steel construction; shell and tube floating head)

CE index for process equipment in 1987

CE index for process equipment in 1995

Installed cost of heat exchanger in 1995 
8. Heat Exchanger for Cooling Shifted Synthesis Gas with

Feedwater

$\mathrm{Q}=$ Load (Btu/h)

Tha $=$ Inlet temperature of hot fluid $\left({ }^{\circ} \mathrm{F}\right)$

$95,260,677$

456

100

$\mathrm{Thb}=$ Outlet temperature of hot fluid $\left({ }^{\circ} \mathrm{F}\right)$

457

Pressure of hot gases (psia)

70

Tca $=$ Inlet temperature of cold fluid $\left({ }^{\circ} \mathrm{F}\right)$

400

Delta T1

Delta T2

56

30

Log mean temperature difference $\left({ }^{\circ} \mathrm{F}\right)$

42

Overall heat transfer coefficient $\left(\mathrm{Btu} / \mathrm{h} / \mathrm{ft} 2 /{ }^{\circ} \mathrm{F}\right)$

50

Heat transfer area $\left(\mathrm{ft}^{2}\right)$

Operating pressure (psia)

45,899

457

Pressure factor

Materials correction factor

1.175

Module factor

1

3.2

(includes all of the supporting equipment and connections and installation)

Purchased cost of heat exchanger in 1987

(mild steel construction; shell and tube floating head)

CE index for process equipment in 1987

CE index for process equipment in 1995

Installed cost of heat exchanger in 1995 
TABLE 5.9 Sizing and Cost Estimation for Major Equipment Used for $\mathrm{CO}_{2}$ Removal in Membrane Process in Case 2

1. First-Stage Membranes

Membrane area $\left(\mathrm{ft}^{2}\right)$

$1,639,589$

Unit cost of membrane

$\$ 13.00$

Total cost

$\$ 21,314,656$

2. Second-Stage Membranes

Membrane area $\left(\mathrm{ft}^{2}\right)$

Unit cost of membrane

414,731

Total cost

$\$ 5,391,500$

3. Compressor between First and Second Stages

Inlet pressure (psia)

Outlet pressure (psia)

45.00

445.00

Compressor size (hp)

10,208

Purchased cost of reciprocating compressor in 1987

(includes electric motor drive and gear reducer)

Size factor for compressor

$\$ 1,600,000$

Materials correction factor

Module factor

CE index for process equipment in 1987

CE index for process equipment in 1995

Installed cost of compressor in 1995

373.9

4. Recycle Compressor

Inlet pressure (psia)

435.00

Outlet pressure (psia)

445.00

Compressor size (hp)

Purchased cost of reciprocating compressor in 1987

$\$ 60,000$

(includes electric motor drive and gear reducer)

Size factor for compressor

Materials correction factor

Module factor

CE index for process equipment in 1987

CE index for process equipment in 1995

373.9

$\$ 4,860,700$

Installed cost of compressor in 1995 
5. Heat Exchanger After Compressor

$Q=\operatorname{Load}(B t u / h)$

Tha $=$ Inlet temperature of hot fluid $\left({ }^{\circ} \mathrm{F}\right)$

$19,238,746$

538.71

212

Thb = Outlet temperature of hot fluid $\left({ }^{\circ} \mathrm{F}\right)$

450

Tca $=$ Inlet temperature of cold fluid $\left({ }^{\circ} \mathrm{F}\right)$

70.00

$\mathrm{Tcb}=$ Outlet temperature of cold fluid $\left({ }^{\circ} \mathrm{F}\right)$

150.00

Delta T1

Delta T2

388.71

142

245

Log mean temperature difference $\left({ }^{\circ} \mathrm{F}\right)$

Overall heat transfer coefficient $\left(\mathrm{Btu} / \mathrm{h} / \mathrm{ft}^{2} /{ }^{\circ} \mathrm{F}\right)$

40

Heat transfer area $\left(\mathrm{ft}^{2}\right)$

Operating pressure (psia)

1,963

445

1.08

Pressure factor

Materials correction factor

Module factor

(includes all of the supporting equipment and connections and installation)

Purchased cost of heat exchanger in 1987

(mild steel construction; shell and tube floating head)

CE index for process equipment in 1987

CE index for process equipment in 1995

Installed cost of heat exchanger in 1995

$\$ 36,000$

320

373.9

$\$ 145,372$

6. $\mathrm{CO}_{2}$ Product Gas Compressors

Compressor 1 (hp)

Compressor 2 (hp)

Compressor 3 (hp)

Purchased cost of centrifugal compressor 1 in 1987

Purchased cost of centrifugal compressor 2 in 1987

Purchased cost of centrifugal compressor 3 in 1987

(includes electric motor drive and gear reducer)

Size factor for compressor

Module factor

2,583

2,583

2,583

$\$ 540,000$

$\$ 540,000$

$\$ 540,000$

CE index for process equipment in 1987

CE index for process equipment in 1995

$\$ 1,640,486$

$\$ 1,640,486$

$\$ 1,640,486$

Installed cost of Compressor 2 in 1995
Installed cost of Compressor 3 in 1995

$\$ 36,815,962$

Total Direct Cost 


\section{Case 3 - Fuel Cell Topping Cycle and Glycol $\mathrm{CO}_{2}$ Recovery}

Because fuel cells require a hydrogen-rich fuel stream, the fuel cell system employs a reformer to convert hydrocarbon fuels to hydrogen-rich fuels. For medium-Btu coal gas, a shift reaction is required to create a hydrogen-rich fuel. Because of the high operating temperature of the molten carbonate fuel cell, a reforming or a shift reaction can take place within the cell, eliminating the need for separate reactors for these processes. The associated economies recommend a fuel cell as the topping cycle for IGCC with $\mathrm{CO}_{2}$ recovery. Material and energy balances have been developed in this section for the application of an internal reforming molten carbonate fuel cell as the topping cycle for an IGCC plant. The $\mathrm{CO}_{2}$ from the fuel cell exhaust is recovered in a glycol process. This situation is quite different from use of a gas turbine topping cycle, in which $\mathrm{CO}_{2}$ recovery must precede use of the fuel in the turbine to avoid dilution with air, which would increase the cost of $\mathrm{CO}_{2}$ recovery.

\subsection{Design Basis}

Figure 6.1 provides an overview of the of the IGCC system, including the gasifier, gas treatment, the fuel cell, and the steam cycle. The overall design of the fuel cell is determined by the gasifier capacity and synthesis gas composition. These are assumed to be the same as in the base case, which has no $\mathrm{CO}_{2}$ recovery. The fuel cell has very low tolerance for contaminants, including particulates and sulfur compounds. To achieve the required level of $\mathrm{H}_{2} \mathrm{~S}$ removal, a chilled methanol system has been employed rather than the glycol system used in the gas turbine cases. The chilled methanol system is designed to reduce the sulfur species $\left(\mathrm{H}_{2} \mathrm{~S}\right.$ and $\left.\mathrm{COS}\right)$ concentration to less than 1 part per million volume (ppmv). The reactions in the fuel cell anode shift the synthesis gas to a hydrogen-rich gas with a high concentration of $\mathrm{CO}_{2}$ and reduce the resultant hydrogen with carbonate ion. Oxidation of the carbonate at the anode releases $\mathrm{CO}_{2}$ and two moles of electrons per mole of $\mathrm{H}_{2}$ converted. The $\mathrm{CO}_{2}$-rich anode exhaust is treated in a glycol recovery system to separate most of the $\mathrm{CO}_{2}$. Thermal energy released by cooling this anode exhaust provides heat for the steam bottoming cycle. An expansion turbine is used on the cathode exhaust to extract energy.

Table 6.1 is a summary of principal material flows for the base case and for this design option. The $\mathrm{CO}_{2}$ reduction accomplished at the power plant is $89 \%$ and is accompanied by a $25 \%$ reduction in net electrical output. A full accounting of the net $\mathrm{CO}_{2}$ reduction would include $\mathrm{CO}_{2}$ released in the generation of replacement power, mining, coal and reagent preparation, and materials transport.

\subsection{Chilled Methanol Process for $\mathrm{H}_{2} \mathrm{~S}$ Recovery}

Because of the extremely low tolerance of the fuel cell for $\mathrm{H}_{2} \mathrm{~S}$, a chilled methanol process has been employed rather than the more economical glycol process preferred for the base 


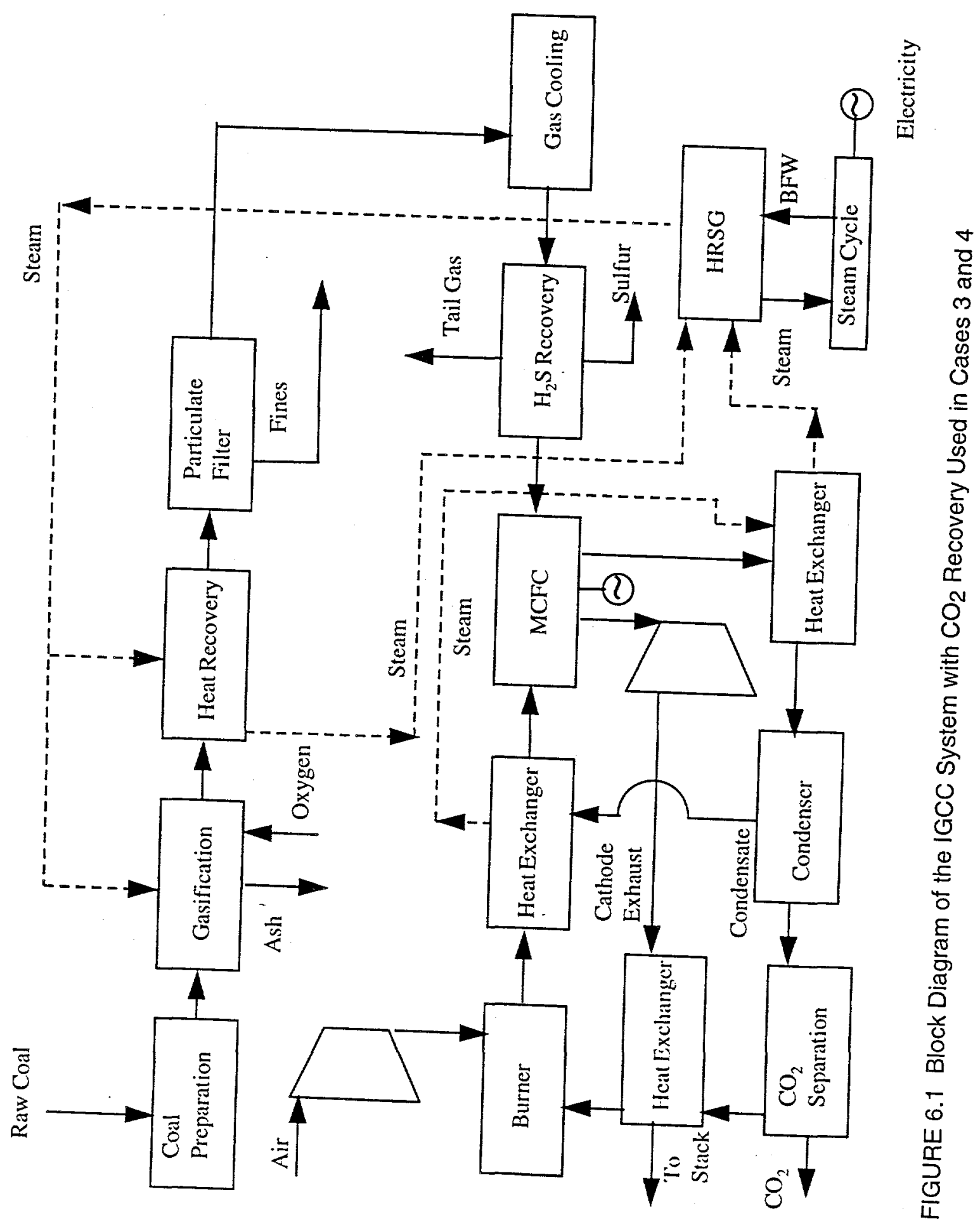


TABLE 6.1 Material Flows for Oxygen-Blown Base Case and Case 3

\begin{tabular}{|c|c|c|}
\hline Material Flow (tons/d) & Base Case & Case 3 \\
\hline Coal (prepared) & 3,845 & 3,845 \\
\hline Oxygen & 2,347 & 2,347 \\
\hline Solid waste & 492 & 492 \\
\hline Sulfur & 78 & 78 \\
\hline $\mathrm{CO}_{2}$ (power plant only) & 9210 & 993 \\
\hline $\mathrm{SO}_{2}$ (power plant only) & 1.08 & 6.92 \\
\hline Net power output (MW) & 458.4 & 340.11 \\
\hline
\end{tabular}

case and gas turbine options. The chilled methanol process is depicted in Figure 6.2. The feed gas is cooled by heat exchange with the cleaned fuel gas. Because it is cooled to well below the point at which water would condense and freeze, methanol is added to the feed gas to act as an antifreeze. Condensate is removed in a phase separater and sent to a distillation unit to recover the methanol. The rich methanol from the absorber is flashed in three stages to release the $\mathrm{H}_{2} \mathrm{~S}$ and is finally stripped with steam heating. The lean methanol from the stripper is cooled by heat exchange with the methanol feed to the stripper and by refrigeration prior to reinjection into the absorber tower. Table 6.2 provides the details of stream composition, flows, and conditions for the $\mathrm{H}_{2} \mathrm{~S}$ recovery system. Comparing the feed stream, 1A, with the product stream, 2B, the reduction in $\mathrm{H}_{2} \mathrm{~S}$ in $\mathrm{lb} \cdot \mathrm{mol} / \mathrm{h}$ is $99.99 \%$ and the $\mathrm{H}_{2} \mathrm{~S}$ content of the fuel gas is about $0.7 \mathrm{ppmv}$. A description of the streams and assumptions used in the stream calculations is provided in Table 6.3.

\subsection{Molten Carbonate Fuel Cell System}

Figure 6.3 shows the molten carbonate fuel cell in the context of supporting systems. The sulfur-free gas from the methanol system is brought to fuel cell operating pressure in a power recovery turbine. The gas is then heated by steam injection and fed to the fuel cell, where the shift reaction converts $\mathrm{CO}$ to $\mathrm{CO}_{2}$ and reforming converts $\mathrm{CH}_{4}$ to $\mathrm{H}_{2}$ and $\mathrm{CO}_{2}$. The anode exhaust is rich in $\mathrm{CO}_{2}$. The sensible heat of this stream is used to raise steam for the steam cycle. After further cooling by heat exchange with steam cycle condensate, the anode exhaust is sent to $\mathrm{CO}_{2}$ recovery following water removal in a condenser. The $\mathrm{CO}_{2}$-lean gas has residual $\mathrm{CO}$ and $\mathrm{H}_{2}$, which is burned in air before this stream is used as the cathode feed. The cathode exhaust is sent through a power recovery turbine and heat exchangers before being exhausted as stack gas. The line list corresponding to Figure 6.3 is provided in Table 6.4. A description of the streams and key assumptions are provided in Table 6.5. 


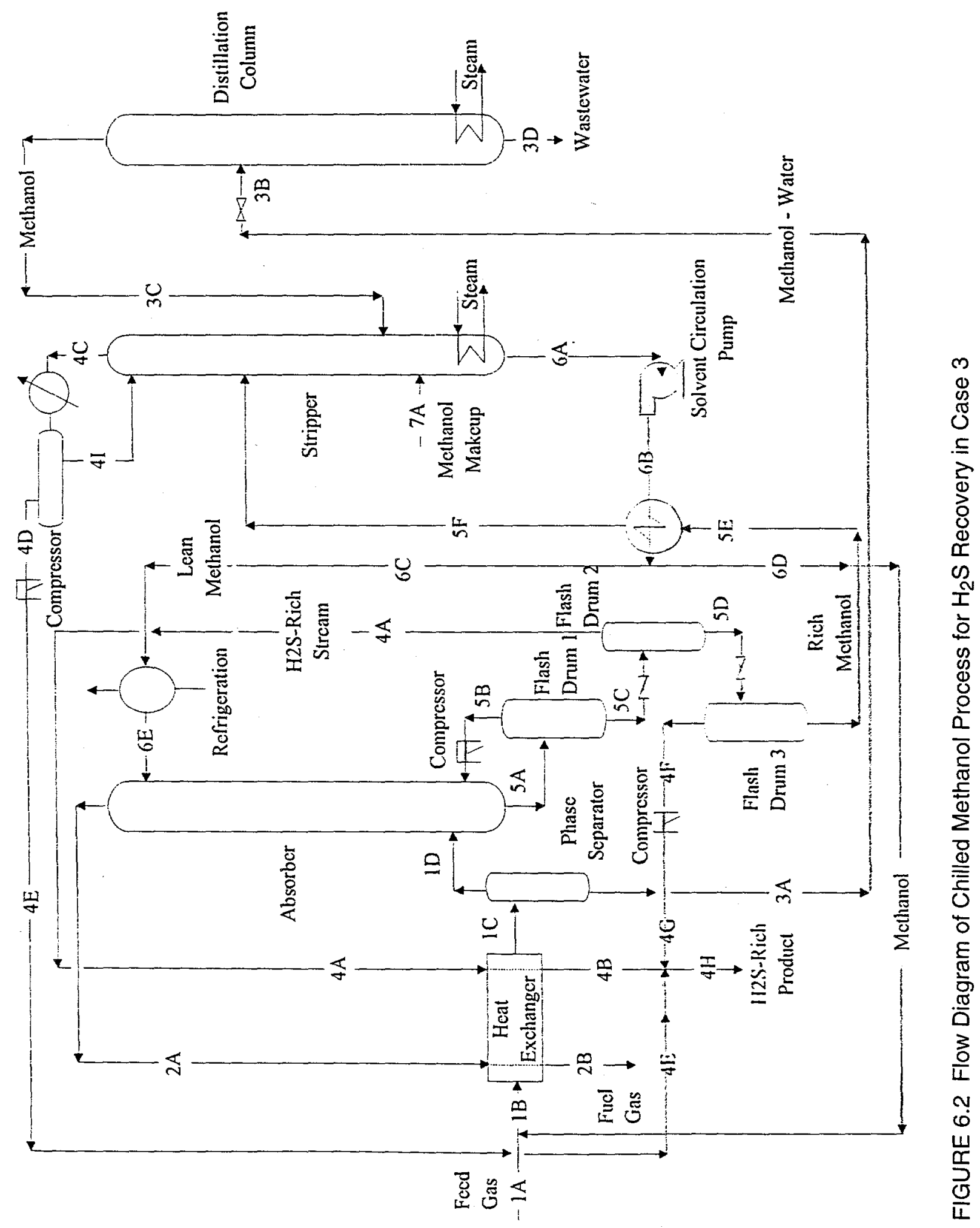


TABLE 6.2 Stream Flows of Chilled Methanol Process for $\mathrm{H}_{2} \mathrm{~S}$ Removal in Case 3

\begin{tabular}{|c|c|c|c|c|c|c|}
\hline Stream Data & Stream 1A & Stream 18 & Stream $1 \mathrm{C}$ & Stream 1D & Stream $2 A$ & Stream 2B \\
\hline Description of stream & $\begin{array}{l}\text { Feed gas from } \\
\text { KRW gasifier }\end{array}$ & $\begin{array}{l}\text { Gases to heat } \\
\text { exchanger }\end{array}$ & $\begin{array}{l}\text { Gases from heat } \\
\text { exchanger }\end{array}$ & $\begin{array}{l}\text { Gases from phase } \\
\text { separator }\end{array}$ & $\begin{array}{l}\text { Sulfur-free gas } \\
\text { from absorber }\end{array}$ & $\begin{array}{l}\text { Sulfur-free gas } \\
\text { to fuel cell }\end{array}$ \\
\hline \multicolumn{7}{|l|}{ Gases $(\mathrm{lb} \cdot \mathrm{mol} / \mathrm{h})$} \\
\hline $\mathrm{CO}$ & $4,559.29$ & $4,559.29$ & $4,559.29$ & $4,559.29$ & $4,530.65$ & $4,530.65$ \\
\hline $\mathrm{CO}_{2}$ & 389.37 & 389.37 & 389.37 & 389.37 & 267.08 & 267.08 \\
\hline $\mathrm{H}_{2}$ & $2,315.44$ & $2,315.44$ & $2,315.44$ & $2,315.44$ & $2,311.51$ & $2,311.51$ \\
\hline $\mathrm{H}_{2} \mathrm{O}$ & 19.41 & 19.41 & 19.41 & 0.00 & 0.00 & 0.00 \\
\hline $\mathrm{N}_{2}$ & 36.44 & 36.44 & 36.44 & 36.44 & 36.44 & 36.44 \\
\hline $\mathrm{Ar}$ & 72.73 & 72.73 & 72.73 & 72.73 & 72.73 & 72.73 \\
\hline $\mathrm{CH}_{4}$ & 487.31 & 487.31 & 487.31 & 487.31 & 480.56 & 480.56 \\
\hline $\mathrm{NH}_{3}$ & 0.00 & 0.00 & 0.00 & 0.00 & 0.01 & 0.01 \\
\hline $\mathrm{H}_{2} \mathrm{~S}$ & 59.01 & 59.01 & 59.01 & 59.01 & $5.90 E-03$ & $5.90 \mathrm{E}-03$ \\
\hline $\mathrm{HCN}$ & 0.00 & 0.00 & 0.00 & 0.00 & 7.42E-04 & 7.42E-04 \\
\hline $\mathrm{O}_{2}$ & 0.00 & 0.00 & 0.00 & 0.00 & 0.00 & 0.00 \\
\hline $\cos$ & 7.42 & 7.42 & 7.42 & 7.42 & 0.00 & 0.00 \\
\hline $\mathrm{SO}_{2}$ & 0.00 & 0.00 & 0.00 & 0.00 & 0.00 & 0.00 \\
\hline Total gas flow & $7,946.42$ & $7,946.42$ & $7,946.42$ & $7,927.01$ & $7,698.98$ & $7,698.98$ \\
\hline $\begin{array}{l}\text { Liquids }(\mathrm{lb} \cdot \mathrm{mol} / \mathrm{h}) \\
\text { Methanol }\end{array}$ & 0.00 & 15.07 & 15.07 & 0.00 & 0.03 & 0.03 \\
\hline Temperature $\left({ }^{\circ} \mathrm{F}\right)$ & 105.00 & 104.55 & -34.18 & -34.18 & -70.00 & 80.00 \\
\hline Pressure (psia) & 456.00 & 456.00 & 456.00 & 456.00 & 450.00 & 450.00 \\
\hline $\begin{array}{l}\text { Enthalpy of stream }(\mathrm{Btu} / \mathrm{h}) \\
\text { (reference, } 32^{\circ} \mathrm{F} \text { ) }\end{array}$ & $4,444,715$ & $4,433,245$ & $-3,707,336$ & $-3,666,146$ & $-5,440,448$ & $2,596,543$ \\
\hline
\end{tabular}


TABLE 6.2 (Cont.)

\begin{tabular}{|c|c|c|c|c|c|c|}
\hline Stream Data & Stream $3 A$ & Stream 3B & Stream $3 C$ & Stream 3D & Stream 4A & Stream 4B \\
\hline Description of stream & $\begin{array}{l}\text { Bottoms from } \\
\text { phase separator }\end{array}$ & $\begin{array}{l}\text { Feed to distillation } \\
\text { column }\end{array}$ & $\begin{array}{l}\text { Overhead from } \\
\text { distillation column }\end{array}$ & $\begin{array}{l}\text { Wastewater from } \\
\text { distillation column }\end{array}$ & $\begin{array}{l}\mathrm{H}_{2} \mathrm{~S} \text {-rich gas } \\
\text { from flash } \\
\text { drum } 2\end{array}$ & $\begin{array}{l}\mathrm{H}_{2} \mathrm{~S} \text {-rich gas from } \\
\text { heat exchanger }\end{array}$ \\
\hline \multicolumn{7}{|l|}{ Gases $(\mathrm{lb} \cdot \mathrm{mol} / \mathrm{h})$} \\
\hline $\mathrm{CO}$ & 0.00 & 0.00 & 0.00 & 0.00 & 21.48 & 21.48 \\
\hline $\mathrm{CO}_{2}$ & 0.00 & 0.00 & 0.00 & 0.00 & 61.14 & 61.14 \\
\hline $\mathrm{H}_{2}$ & 0.00 & 0.00 & 0.00 & 0.00 & 3.14 & 3.14 \\
\hline $\mathrm{H}_{2} \mathrm{O}$ & 19.41 & 19.41 & 0.79 & 18.62 & 0.00 & 0.00 \\
\hline $\mathrm{N}_{2}$ & 0.00 & 0.00 & 0.00 & 0.00 & 0.00 & 0.00 \\
\hline $\operatorname{Ar}$ & 0.00 & 0.00 & 0.00 & 0.00 & 0.00 & 0.00 \\
\hline $\mathrm{CH}_{4}$ & 0.00 & 0.00 & 0.00 & 0.00 & 4.05 & 4.05 \\
\hline $\mathrm{NH}_{3}$ & 0.00 & 0.00 & 0.00 & 0.00 & 0.00 & 0.00 \\
\hline $\mathrm{H}_{2} \mathrm{~S}$ & 0.00 & 0.00 & 0.00 & 0.00 & 17.83 & 17.83 \\
\hline $\mathrm{HCN}$ & 0.00 & 0.00 & 0.00 & 0.00 & 0.00 & 0.00 \\
\hline $\mathrm{O}_{2}$ & 0.00 & 0.00 & 0.00 & 0.00 & 0.00 & 0.00 \\
\hline $\cos$ & 0.00 & 0.00 & 0.00 & 0.00 & 2.35 & 2.35 \\
\hline $\mathrm{SO}_{2}$ & 0.00 & 0.00 & 0.00 & 0.00 & 0.00 & 0.00 \\
\hline Total gas flow & 19.41 & 19.41 & 0.79 & 18.62 & 109.99 & 109.99 \\
\hline $\begin{array}{l}\text { Liquids (lb.mol/h) } \\
\text { Methanol }\end{array}$ & 15.07 & 15.07 & 15.07 & 0.00 & 0.00 & 0.00 \\
\hline Temperature $\left({ }^{\circ} \mathrm{F}\right)$ & -34.18 & -34.18 & 150.00 & 280.00 & -29.88 & 84.50 \\
\hline Pressure (psia) & 456.00 & 50.00 & 50.00 & 50.00 & 150.00 & 150.00 \\
\hline $\begin{array}{l}\text { Enthalpy of stream }(\mathrm{Btu} / \mathrm{h}) \\
\left.\text { (reference, } 32^{\circ} \mathrm{F}\right)\end{array}$ & $-41,190$ & $-41,190$ & 270,074 & 83,134 & $-55,403$ & 48,168 \\
\hline
\end{tabular}


TABLE 6.2 (Cont.)

\begin{tabular}{|c|c|c|c|c|c|c|}
\hline Stream Data & Stream $4 \mathrm{C}$ & Stream 4D & Stream 4E & Stream 4F & Stream 4G & Stream $4 \mathrm{H}$ \\
\hline Description of stream & $\begin{array}{l}\text { Overhead from } \\
\text { stripper }\end{array}$ & $\begin{array}{l}\mathrm{H}_{2} \mathrm{~S} \text {-rich gas from } \\
\text { phase separator }\end{array}$ & $\begin{array}{l}\mathrm{H}_{2} \mathrm{~S} \text {-rich gas after } \\
\text { compressor }\end{array}$ & $\begin{array}{l}\mathrm{H}_{2} \mathrm{~S} \text {-rich gas from } \\
\text { flash drum } 3\end{array}$ & $\begin{array}{l}\mathrm{H}_{2} \mathrm{~S} \text {-rich gas after } \\
\text { compressor }\end{array}$ & $\mathrm{H}_{2} \mathrm{~S}$-rich product \\
\hline \multicolumn{7}{|l|}{ Gases (lb·mol/h) } \\
\hline $\mathrm{CO}$ & 1.79 & 1.79 & 1.79 & 5.37 & 5.37 & 28.64 \\
\hline $\mathrm{CO}_{2}$ & 30.57 & 30.57 & 30.57 & 30.57 & 30.57 & 122.29 \\
\hline $\mathrm{H}_{2}$ & 0.16 & 0.16 & 0.16 & 0.63 & 0.63 & 3.93 \\
\hline $\mathrm{H}_{2} \mathrm{O}$ & 0.79 & 0.79 & 0.79 & 0.00 & 0.00 & 0.79 \\
\hline $\mathrm{N}_{2}$ & 0.00 & 0.00 & 0.00 & 0.00 & 0.00 & 0.00 \\
\hline $\operatorname{Ar}$ & 0.00 & 0.00 & 0.00 & 0.00 & 0.00 & 0.00 \\
\hline $\mathrm{CH}_{4}$ & 1.35 & 1.35 & 1.35 & 1.35 & 1.35 & 6.75 \\
\hline $\mathrm{NH}_{3}$ & 0.00 & 0.00 & 0.00 & 0.00 & 0.00 & 0.00 \\
\hline $\mathrm{H}_{2} \mathrm{~S}$ & 28.70 & 28.70 & 28.70 & 12.48 & 12.48 & 59.01 \\
\hline $\mathrm{HCN}$ & 0.00 & 0.00 & 0.00 & 0.00 & 0.00 & 0.00 \\
\hline $\mathrm{O}_{2}$ & 0.00 & 0.00 & 0.00 & 0.00 & 0.00 & 0.00 \\
\hline $\cos$ & 3.42 & 3.42 & 3.42 & 1.64 & 1.64 & 7.42 \\
\hline $\mathrm{SO}_{2}$ & 0.00 & 0.00 & 0.00 & 0.00 & 0.00 & 0.00 \\
\hline Total gas flow & 66.78 & 66.78 & 66.78 & 52.04 & 52.04 & 228.83 \\
\hline $\begin{array}{l}\text { Liquids }(\mathrm{lb} \cdot \mathrm{mol} / \mathrm{h}) \\
\text { Methanol }\end{array}$ & 224.97 & 21.45 & 21.45 & 0.00 & 0.00 & 21.45 \\
\hline Temperature $\left({ }^{\circ} \mathrm{F}\right)$ & 135.00 & 100.00 & 619.94 & -33.64 & 286.22 & 318.59 \\
\hline Pressure (psia) & 14.70 & 14.70 & 150.00 & 20.00 & 150.00 & 95.00 \\
\hline $\begin{array}{l}\text { Enthalpy of stream }(B t u / h) \\
\left.\text { (reference, } 32^{\circ} \mathrm{F}\right)\end{array}$ & $3,874,837$ & 410,887 & 803,850 & $-28,354$ & 117,525 & 969,544 \\
\hline
\end{tabular}


TABLE 6.2 (Cont.)

\begin{tabular}{|c|c|c|c|c|c|c|}
\hline Stream Data & Stream 41 & Stream $5 \mathrm{~A}$ & Stream 5B & Stream 5C & Stream 5D & Stream $5 E$ \\
\hline Description of stream & $\begin{array}{l}\text { Methanol reflux to } \\
\text { stripper }\end{array}$ & $\begin{array}{l}\text { Rich methanol from } \\
\text { absorber }\end{array}$ & $\begin{array}{l}\text { Gases from flash } \\
\text { drum } 1\end{array}$ & $\begin{array}{l}\text { Feed to flash } \\
\text { drum } 2\end{array}$ & $\begin{array}{l}\text { Feed to flash } \\
\text { drum } 3\end{array}$ & $\begin{array}{l}\text { Rich methanol from } \\
\text { flash drum } 3\end{array}$ \\
\hline \multicolumn{7}{|l|}{ Gases (lb.mol/h) } \\
\hline CO & 0.00 & 286.38 & 257.74 & 28.64 & 7.16 & 1.79 \\
\hline $\mathrm{CO}_{2}$ & 0.00 & 152.86 & 30.57 & 122.29 & 61.14 & 30.57 \\
\hline $\mathrm{H}_{2}$ & 0.00 & 39.27 & 35.34 & 3.93 & 0.79 & 0.16 \\
\hline $\mathrm{H}_{2} \mathrm{O}$ & 0.00 & 0.00 & 0.00 & 0.00 & 0.00 & 0.00 \\
\hline $\mathrm{N}_{2}$ & 0.00 & 0.00 & 0.00 & 0.00 & 0.00 & 0.00 \\
\hline $\mathrm{Ar}$ & 0.00 & 0.00 & 0.00 & 0.00 & 0.00 & 0.00 \\
\hline $\mathrm{CH}_{4}$ & 0.00 & 67.52 & 60.77 & 6.75 & 2.70 & 1.35 \\
\hline $\mathrm{NH}_{3}$ & 0.00 & 0.00 & 0.00 & 0.00 & 0.00 & 0.00 \\
\hline $\mathrm{H}_{2} \mathrm{~S}$ & 0.00 & 66.02 & 6.60 & 59.42 & 41.60 & 29.12 \\
\hline $\mathrm{HCN}$ & 0.00 & 0.00 & 0.00 & 0.00 & 0.00 & 0.00 \\
\hline $\mathrm{O}_{2}$ & 0.00 & 0.00 & 0.00 & 0.00 & 0.00 & 0.00 \\
\hline $\cos$ & 0.00 & 8.70 & 0.87 & 7.83 & 5.48 & 3.84 \\
\hline $\mathrm{SO}_{2}$ & 0.00 & 0.00 & 0.00 & 0.00 & 0.00 & 0.00 \\
\hline Total gas flow & 0.00 & 620.75 & 391.89 & 228.86 & 118.87 & 66.83 \\
\hline $\begin{array}{l}\text { Liquids }(\mathrm{lb} \cdot \mathrm{mol} / \mathrm{h}) \\
\text { Methanol }\end{array}$ & 203.53 & $4,114.03$ & 0.00 & $4,114.03$ & $4,114.03$ & $4,114.03$ \\
\hline Temperature $\left({ }^{\circ} \mathrm{F}\right)$ & 100.00 & -23.04 & -23.04 & -23.04 & -29.88 & -33.64 \\
\hline Pressure (psia) & 95.00 & 450.00 & 300.00 & 300.00 & 150.00 & 20.00 \\
\hline $\begin{array}{l}\text { Enthalpy of stream }(\mathrm{Btu} / \mathrm{h}) \\
\left.\text { (reference, } 32^{\circ} \mathrm{F}\right)\end{array}$ & 250,640 & $-4,358,335$ & $-153,557$ & $-4,204,777$ & $-4,671,853$ & $-4,927,451$ \\
\hline
\end{tabular}


TABLE 6.2 (Cont.)

\begin{tabular}{|c|c|c|c|c|c|c|c|}
\hline Stream Data & Stream $5 F$ & Stream 6A & Stream 6B & Stream 6C & Stream 6D & Stream $6 E$ & Stream 7A \\
\hline Description of stream & $\begin{array}{l}\text { Rich methanol } \\
\text { to stripper }\end{array}$ & $\begin{array}{l}\text { Lean methanol } \\
\text { to circulation } \\
\text { pump }\end{array}$ & $\begin{array}{l}\text { Lean methanol } \\
\text { from solvent } \\
\text { circulation pump }\end{array}$ & $\begin{array}{l}\text { Lean methano! } \\
\text { to refrigeration }\end{array}$ & $\begin{array}{l}\text { Methanol for } \\
\text { feed gas } \\
\text { injection }\end{array}$ & $\begin{array}{l}\text { Lean methanol } \\
\text { to absorber }\end{array}$ & $\begin{array}{l}\text { Methanol } \\
\text { makeup to } \\
\text { stripper }\end{array}$ \\
\hline \multicolumn{8}{|l|}{ Gases $(\mathrm{lb} \cdot \mathrm{mol} / \mathrm{h})$} \\
\hline $\mathrm{CO}$ & 1.79 & 0.00 & 0.00 & 0.00 & 0.00 & 0.00 & 0.00 \\
\hline $\mathrm{CO}_{2}$ & 30.57 & 0.00 & 0.00 & 0.00 & 0.00 & 0.00 & 0.00 \\
\hline $\mathrm{H}_{2}$ & 0.16 & 0.00 & 0.00 & 0.00 & 0.00 & 0.00 & 0.00 \\
\hline $\mathrm{H}_{2} \mathrm{O}$ & 0.00 & 0.00 & 0.00 & 0.00 & 0.00 & 0.00 & 0.00 \\
\hline $\mathrm{N}_{2}$ & 0.00 & 0.00 & 0.00 & 0.00 & 0.00 & 0.00 & 0.00 \\
\hline $\mathrm{Ar}$ & 0.00 & 0.00 & 0.00 & 0.00 & 0.00 & 0.00 & 0.00 \\
\hline $\mathrm{CH}_{4}$ & 1.35 & 0.00 & 0.00 & 0.00 & 0.00 & 0.00 & 0.00 \\
\hline $\mathrm{NH}_{3}$ & 0.00 & 0.00 & 0.00 & 0.00 & 0.00 & 0.00 & 0.00 \\
\hline $\mathrm{H}_{2} \mathrm{~S}$ & 29.12 & 0.41 & 0.41 & 0.41 & 0.00 & 0.41 & 0.00 \\
\hline $\mathrm{HCN}$ & 0.00 & 0.00 & 0.00 & 0.00 & 0.00 & 0.00 & 0.00 \\
\hline $\mathrm{O}_{2}$ & 0.00 & 0.00 & 0.00 & 0.00 & 0.00 & 0.00 & 0.00 \\
\hline $\cos$ & 3.84 & 0.41 & 0.41 & 0.41 & 0.00 & 0.41 & 0.00 \\
\hline $\mathrm{SO}_{2}$ & 0.00 & 0.00 & 0.00 & 0.00 & 0.00 & 0.00 & 0.00 \\
\hline Total gas flow & 66.83 & 0.82 & 0.82 & 0.82 & 0.00 & 0.82 & 0.00 \\
\hline $\begin{array}{l}\text { Liquids }(\mathrm{lb} \cdot \mathrm{mol} / \mathrm{h}) \\
\text { Methanol }\end{array}$ & $4,114.03$ & $4,129.14$ & $4,129.14$ & $4,114.06$ & 15.07 & $4,114.06$ & 21.48 \\
\hline Temperature $\left({ }^{\circ} \mathrm{F}\right)$ & 128.66 & 149.00 & 152.90 & -10.00 & -10.00 & -70.00 & 70.00 \\
\hline Pressure (psia) & 20.00 & 14.70 & 456.00 & 456.00 & 456.00 & 456.00 & 14.70 \\
\hline $\begin{array}{l}\text { Enthalpy of stream }(\mathrm{Btu} / \mathrm{h}) \\
\text { (reference, } 32^{\circ} \mathrm{F} \text { ) }\end{array}$ & $7,257,493$ & $8,749,976$ & $9,043,938$ & $-3,129,543$ & $-11,463$ & $-7,600,310$ & 14,782 \\
\hline
\end{tabular}


TABLE 6.3 Descriptions of Streams of Chilled Methanol Process for $\mathrm{H}_{2} \mathrm{~S}$ Removal in Case 3

\begin{tabular}{ccc}
\hline $\begin{array}{c}\text { Stream and } \\
\text { Characteristics }\end{array}$ & Data & Comments on Stream Calculations \\
\hline
\end{tabular}

Stream 1A: Gas feed from KRW process

Temperature ( $\left.{ }^{\circ} \mathrm{F}\right)$

Pressure (psia)

Flow rate $(\mathrm{lb} \cdot \mathrm{mol} / \mathrm{h})$

$\mathrm{H}_{2} \mathrm{~S}$ (mol fraction)

Stream 2A: Fuel gas from top of absorber

Temperature $\left({ }^{\circ} \mathrm{F}\right)$

Pressure (psia)

Flow rate $(\mathrm{lb} \cdot \mathrm{mol} / \mathrm{h})$

$\mathrm{H}_{2} \mathrm{~S}$ (ppm)

Stream 3A: Methanol-water mixture

from phase separator

Temperature $\left({ }^{\circ} \mathrm{F}\right)$

Pressure (psia)

Flow rate $(\mathrm{lb} \cdot \mathrm{mol} / \mathrm{h})$

$\mathrm{H}_{2} \mathrm{~S}$ (mol fraction)

Stream 3B: Methanol-water mixture to distillation column

Temperature $\left({ }^{\circ} \mathrm{F}\right)$

Pressure (psia)

Flow rate $(\mathrm{lb} \cdot \mathrm{mol} / \mathrm{h})$

$\mathrm{H}_{2} \mathrm{~S}$ (mol fraction)

Stream 3C: Methanol from distillation

column to stripper

Temperature $\left({ }^{\circ} \mathrm{F}\right)$

Pressure (psia)

Flow rate $(\mathrm{lb} \cdot \mathrm{mol} / \mathrm{h})$

$\mathrm{H}_{2} \mathrm{~S}$ (mol fraction)

Stream 3D: Wastewater from

distillation column

Temperature $\left({ }^{\circ} \mathrm{F}\right)$

Pressure (psia)

Flow rate $(\mathrm{lb} \cdot \mathrm{mol} / \mathrm{h})$

$\mathrm{H}_{2} \mathrm{~S}$ (mol fraction)

Stream 4A: $\mathrm{H}_{2} \mathrm{~S}$-rich gas from

flash drum 2

Temperature $\left({ }^{\circ} \mathrm{F}\right)$

Pressure (psia)

Flow rate $(\mathrm{lb} \cdot \mathrm{mol} / \mathrm{h})$

$\mathrm{H}_{2} \mathrm{~S}$ (mol fraction)
This stream is coming from KRW process.

This stream will be cooled against cold fuel gas from absorber and cold $\mathrm{H}_{2} \mathrm{~S}$-rich gas from flash drum 2.

Chilled methanol enters the top of the column at a temperature of $-70^{\circ} \mathrm{F}$. Gases leaving the column are in equilibrium with methanol; hence, they are at a temperature of $-70^{\circ} \mathrm{F}$. Gas composition corresponds to $99.99 \%$ removal of $\mathrm{H}_{2} \mathrm{~S}$.

Methanol is added to feed gas prior to absorption column to prevent icing of water in feed gas. Condensed water and methanol are separated from gas in phase separator.

Methanol is separated from the methanol-water mixture in distillation column.

Methanol from distillation column is sent to stripper.

Water from distillation column is removed from bottom of column for disposal.

Rich methanol from flash drum 1 is flashed to pressure of 150 psia to desorb major portion of $\mathrm{H}_{2} \mathrm{~S}$ from solvent. 
TABLE 6.3 (Cont.)

\begin{tabular}{ccc}
\hline $\begin{array}{c}\text { Stream and } \\
\text { Characteristics }\end{array}$ & Data & Comments on Stream Calculations \\
\hline
\end{tabular}

Stream 4C: $\mathrm{H}_{2} \mathrm{~S}$-rich gas from stripper

Temperature $\left({ }^{\circ} \mathrm{F}\right)$

Pressure (psia)

Flow rate (lb.mol/h)

$\mathrm{H}_{2} \mathrm{~S}$ (mol fraction)

Stream 4D: $\mathrm{H}_{2}$ S-rich gas from

phase separator

Temperature $\left({ }^{\circ} \mathrm{F}\right)$

Pressure (psia)

Flow rate $(\mathrm{lb} \cdot \mathrm{mol} / \mathrm{h})$

$\mathrm{H}_{2} \mathrm{~S}$ (mol fraction)

Stream 4F: $\mathrm{H}_{2} \mathrm{~S}$-rich gas from

flash drum 3

Temperature $\left({ }^{\circ} \mathrm{F}\right)$

Pressure (psia)

Flow rate $(\mathrm{lb} \cdot \mathrm{mol} / \mathrm{h})$

$\mathrm{H}_{2} \mathrm{~S}$ (mol fraction)

Stream 4H: Final $\mathrm{H}_{2} \mathrm{~S}$-rich product

Temperature ( $\left.{ }^{\circ} \mathrm{F}\right)$

Pressure (psia)

Flow rate $(\mathrm{lb} \cdot \mathrm{mol} / \mathrm{h})$

$\mathrm{H}_{2} \mathrm{~S}$ (mol fraction)

Stream 5A: Rich methanol from

the absorber

Temperature $\left({ }^{\circ} \mathrm{F}\right)$

Pressure (psia)

Flow rate $(\mathrm{lb} \cdot \mathrm{mol} / \mathrm{h})$

$\mathrm{H}_{2} \mathrm{~S}$ (mol fraction)

Stream 5B: Recycle to absorption tower

Temperature $\left({ }^{\circ} \mathrm{F}\right)$

Pressure (psia)

Flow rate $(\mathrm{lb} \cdot \mathrm{mol} / \mathrm{h})$

$\mathrm{H}_{2} \mathrm{~S}$ (mol fraction)

Stream 6A: Lean methanol from stripper

Temperature $\left({ }^{\circ} \mathrm{F}\right)$

Pressure (psia)

Flow rate $(\mathrm{lb} \cdot \mathrm{mol} / \mathrm{h})$

$\mathrm{H}_{2} \mathrm{~S}$ (mol fraction)
The final removal of $\mathrm{H}_{2} \mathrm{~S}$ is achieved in stripper by heat. Because of low vapor pressure of methanol, substantial amounts of methanol will be vaporized along with value of $\mathrm{H}_{2} \mathrm{~S}$.

Methanol is condensed from $\mathrm{H}_{2} \mathrm{~S}$-methanol mixture, and $\mathrm{H}_{2} \mathrm{~S}$ is separated in phase separator.

Rich methanol solution from flash drum 2 is further flashed to pressure of 20 psia in flash drum 3 to desorb $\mathrm{H}_{2} \mathrm{~S}$ from solvent.

The $\mathrm{H}_{2} \mathrm{~S}$-rich streams from stripper and flash drum 3 are compressed to pressure of 95 psia and then combined with $\mathrm{H}_{2} \mathrm{~S}$-rich stream from flash drum 2. This stream is further processed in a Claus plant for sulfur recovery.

Rich methanol, which contains $\mathrm{H}_{2} \mathrm{~S}$ and other soluble gases, is withdrawn from bottom of tower. Temperature of solvent rises because of heat of absorption of $\mathrm{H}_{2} \mathrm{~S}$ into methanol.

Rich methanol is flashed to pressure of 300 psia to desorb gases like $\mathrm{H}_{2}$ and $\mathrm{CH}_{4}$, and the desorbed gases are recycled to absorption tower.

Lean methanol from stripper bottom is to be circulated to absorption tower. The $\mathrm{H}_{2} \mathrm{~S}$ content in lean methanol is 0.0001 moles of $\mathrm{H}_{2} \mathrm{~S}$ per mole of methanol. 
TABLE 6.3 (Cont.)

\begin{tabular}{|c|c|c|}
\hline $\begin{array}{l}\text { Stream and } \\
\text { Characteristics }\end{array}$ & Data & Comments on Stream Calculations \\
\hline \multicolumn{3}{|c|}{$\begin{array}{l}\text { Stream } 6 B \text { : Lean methanol from } \\
\text { circulation pump }\end{array}$} \\
\hline Temperature $\left({ }^{\circ} \mathrm{F}\right)$ & 152.9 & Lean methanol from stripper is at pressure of \\
\hline Pressure (psia) & 456 & 14.7 psia and is pressurized to absorption tower \\
\hline Flow rate $(\mathrm{lb} \cdot \mathrm{mol} / \mathrm{h})$ & $4,129.96$ & operating pressure of 456 psia by using \\
\hline $\mathrm{H}_{2} \mathrm{~S}$ (mol fraction) & 0.0001 & circulation pump. \\
\hline \multicolumn{3}{|c|}{$\begin{array}{l}\text { Stream 6C: Lean methanol from } \\
\text { heat exchanger }\end{array}$} \\
\hline Temperature $\left({ }^{\circ} \mathrm{F}\right)$ & -10 & Lean methanol from circulating pump is cooled \\
\hline Pressure (psia) & 456 & against cold rich methanol from flash drum 3 to \\
\hline Flow rate $(\mathrm{lb} \cdot \mathrm{mol} / \mathrm{h})$ & $4,114.89$ & temperature of $-10^{\circ} \mathrm{F}$. Small portion of methanol \\
\hline $\mathrm{H}_{2} \mathrm{~S}$ (mol fraction) & 0.0001 & $\begin{array}{l}\text { is injected into feed gas prior to absorption to } \\
\text { prevent icing of water. }\end{array}$ \\
\hline \multicolumn{3}{|c|}{ Stream 6E: Lean methanol to stripper } \\
\hline Temperature $\left({ }^{\circ} \mathrm{F}\right)$ & -70 & Lean methanol from heat exchanger is further \\
\hline Pressure (psia) & 456 & cooled to temperature of $-70^{\circ} \mathrm{F}$ by refrigeration. \\
\hline Flow rate $(\mathrm{lb} \cdot \mathrm{mol} / \mathrm{h})$ & $4,114.89$ & 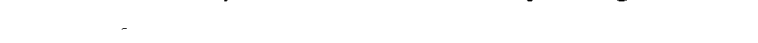 \\
\hline $\mathrm{H}_{2} \mathrm{~S}$ (mol fraction) & 0.0001 & \\
\hline \multicolumn{3}{|l|}{ Stream 7A: Methanol makeup } \\
\hline Temperature $\left({ }^{\circ} \mathrm{F}\right)$ & 70 & Methanol has low vapor pressure; hence, it is \\
\hline Pressure (psia) & 14.7 & lost in stripper along with $\mathrm{H}_{2} \mathrm{~S}$. Also, some \\
\hline Flow rate $(\mathrm{lb} \cdot \mathrm{mol} / \mathrm{h})$ & 21.48 & methanol is lost in distillation column along with \\
\hline $\mathrm{H}_{2} \mathrm{~S}$ (mol fraction) & 0.0 & wastewater. \\
\hline
\end{tabular}




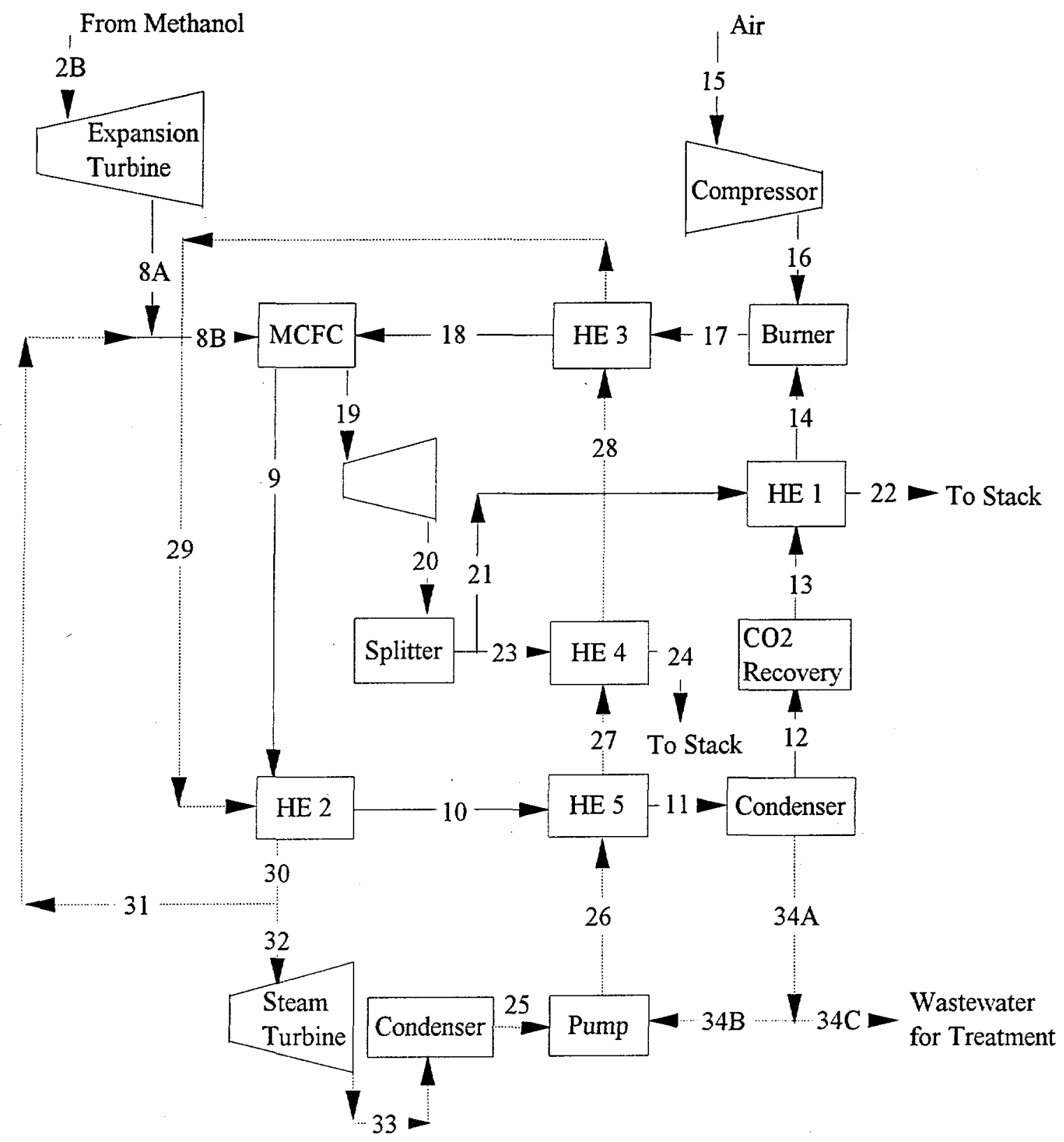

FIGURE 6.3 Flow Diagram of Fuel Cell System and Associated Heat Recovery in Case 3 
TABLE 6.4 Stream Flows of Molten Carbonate Fuel Cell System in Case 3

\begin{tabular}{|c|c|c|c|c|c|c|}
\hline Stream Data & Stream $2 B$ & Stream $8 A$ & Stream 8B & Stream 9 & Stream 10 & Stream 11 \\
\hline Description of stream & $\begin{array}{l}\text { Feed gas from } \\
\text { methanol }\end{array}$ & $\begin{array}{l}\text { Fuel gas from } \\
\text { expansion turbine }\end{array}$ & $\begin{array}{l}\text { Fuel gas to fuel } \\
\text { cell }\end{array}$ & $\begin{array}{l}\text { Fuel cell anode } \\
\text { exhaust }\end{array}$ & $\begin{array}{l}\text { Gases from heat } \\
\text { exchanger } 2\end{array}$ & $\begin{array}{l}\text { Gases from heat } \\
\text { exchanger } 5\end{array}$ \\
\hline \multicolumn{7}{|l|}{ Gases (lb.mol/h) } \\
\hline $\mathrm{CO}$ & $4,530.65$ & $4,530.65$ & $4,530.65$ & $1,812.26$ & $1,812.26$ & $1,812.26$ \\
\hline $\mathrm{CO}_{2}$ & 267.08 & 267.08 & 267.08 & $8,724.66$ & $8,724.66$ & $8,724.66$ \\
\hline $\mathrm{H}_{2}$ & $2,311.51$ & $2,311.51$ & $2,311.51$ & $1,693.50$ & $1,693.50$ & $1,693.50$ \\
\hline $\mathrm{H}_{2} \mathrm{O}$ & 0.00 & 0.00 & $12,000.00$ & $13,579.13$ & $13,579.13$ & $13,579.13$ \\
\hline $\mathrm{N}_{2}$ & 36.44 & 36.44 & 36.44 & 36.44 & 36.44 & 36.44 \\
\hline $\mathrm{Ar}$ & 72.73 & 72.73 & 72.73 & 72.73 & 72.73 & 72.73 \\
\hline $\mathrm{CH}_{4}$ & 480.56 & 480.56 & 480.56 & 0.00 & 0.00 & 0.00 \\
\hline $\mathrm{NH}_{3}$ & 0.00 & 0.00 & 0.00 & 0.00 & 0.00 & 0.00 \\
\hline $\mathrm{H}_{2} \mathrm{~S}$ & $0.8 \mathrm{ppm}$ & $0.8 \mathrm{ppm}$ & $0.8 \mathrm{ppm}$ & 0.00 & 0.00 & 0.00 \\
\hline $\mathrm{HCN}$ & 0.00 & 0.00 & 0.00 & 0.00 & 0.00 & 0.00 \\
\hline $\mathrm{O}_{2}$ & 0.00 & 0.00 & 0.00 & 0.00 & 0.00 & 0.00 \\
\hline $\cos$ & $0.1 \mathrm{ppm}$ & $0.1 \mathrm{ppm}$ & $0.1 \mathrm{ppm}$ & 0.00 & 0.00 & 0.00 \\
\hline $\mathrm{SO}_{2}$ & 0.00 & 0.00 & 0.00 & 0.00 & 0.00 & 0.00 \\
\hline Total gas flow & $7,698.98$ & $7,698.98$ & $19,698.97$ & $25,918.72$ & $25,918.72$ & $25,918.72$ \\
\hline $\begin{array}{l}\text { Liquids }(\mathrm{lb} \cdot \mathrm{mol} / \mathrm{h}) \\
\qquad \mathrm{H}_{2} \mathrm{O}\end{array}$ & 0.00 & 0.00 & 0.00 & 0.00 & 0.00 & 0.00 \\
\hline Temperature $\left({ }^{\circ} \mathrm{F}\right)$ & 80.00 & -28.67 & 502.23 & $1,300.00$ & 450.00 & 150.00 \\
\hline Pressure (psia) & 450.00 & 150.00 & 150.00 & 150.00 & 150.00 & 150.00 \\
\hline $\begin{array}{l}\text { Enthalpy of stream }(\mathrm{Btu} / \mathrm{h}) \\
\text { (reference, } 32^{\circ} \mathrm{F} \text { ) }\end{array}$ & $2,596,057$ & $-3,248,936$ & $302,507,570$ & $591,791,666$ & $351,871,758$ & $41,118,662$ \\
\hline
\end{tabular}


TABLE 6.4 (Cont.)

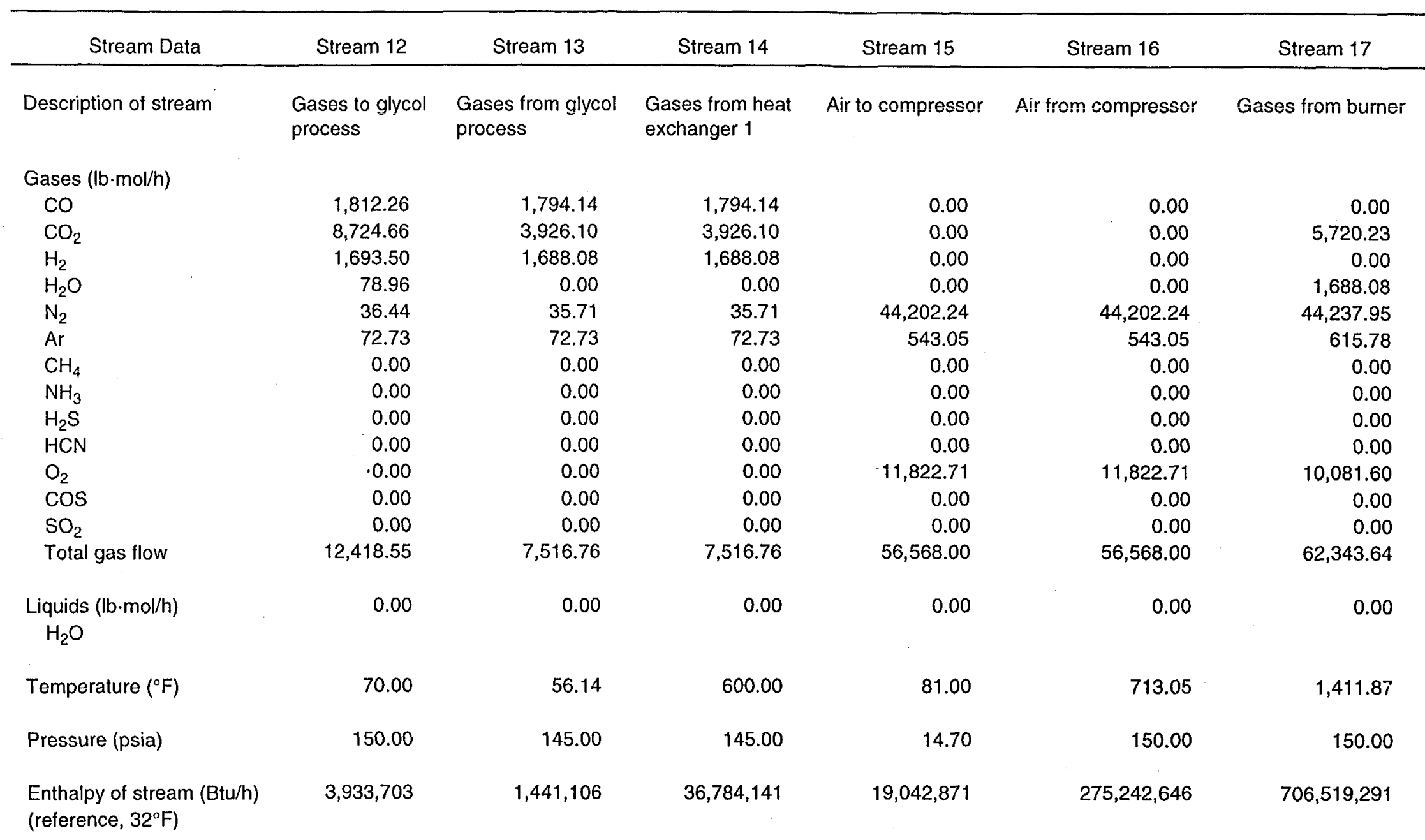


TABLE 6.4 (Cont.)

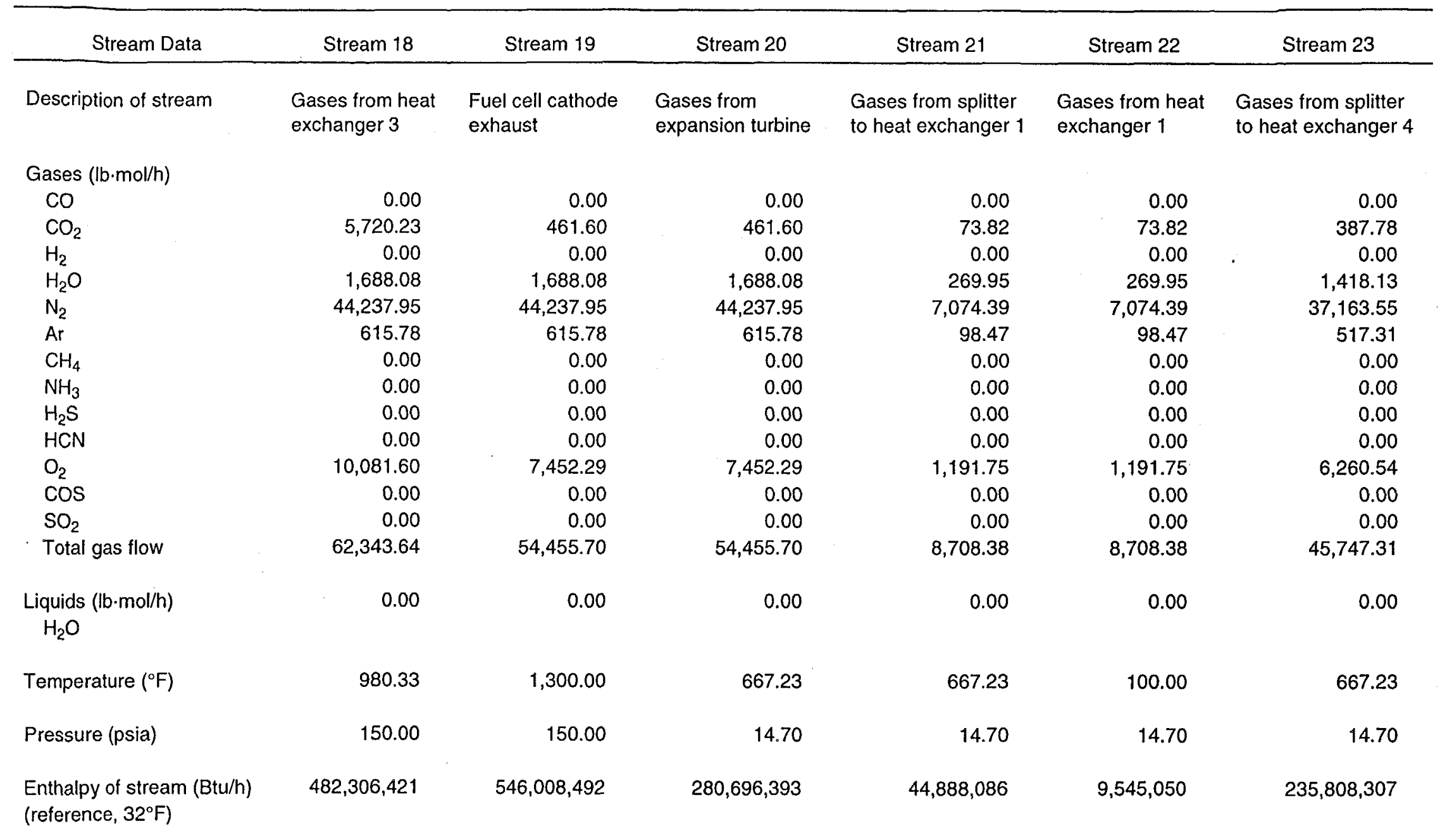


TABLE 6.4 (Cont.)

\begin{tabular}{|c|c|c|c|c|c|c|}
\hline Stream Data & Stream 24 & Stream 25 & Stream 26 & Stream 27 & Stream 28 & Stream 29 \\
\hline Description of stream & $\begin{array}{l}\text { Gases from heat } \\
\text { exchanger } 4\end{array}$ & $\begin{array}{l}\text { Water from } \\
\text { condenser }\end{array}$ & Water from pump & $\begin{array}{l}\text { Steam from heat } \\
\text { exchanger } 5\end{array}$ & $\begin{array}{l}\text { Steam from heat } \\
\text { exchanger } 4\end{array}$ & $\begin{array}{l}\text { Steam from heat } \\
\text { exchanger } 3\end{array}$ \\
\hline \multicolumn{7}{|l|}{ Gases (lb.mol/h) } \\
\hline $\mathrm{CO}$ & 0.00 & 0.00 & 0.00 & 0.00 & 0.00 & 0.00 \\
\hline $\mathrm{CO}_{2}$ & 387.78 & 0.00 & 0.00 & 0.00 & 0.00 & 0.00 \\
\hline $\mathrm{H}_{2}$ & 0.00 & 0.00 & 0.00 & 0.00 & 0.00 & 0.00 \\
\hline $\mathrm{H}_{2} \mathrm{O}$ & $1,418.13$ & 0.00 & 0.00 & $9,917.77$ & $15,668.16$ & $30,055.55$ \\
\hline $\mathrm{N}_{2}$ & $37,163.55$ & 0.00 & 0.00 & 0.00 & 0.00 & 0.00 \\
\hline Ar & 517.31 & 0.00 & 0.00 & 0.00 & 0.00 & 0.00 \\
\hline $\mathrm{CH}_{4}$ & 0.00 & 0.00 & 0.00 & 0.00 & 0.00 & 0.00 \\
\hline $\mathrm{NH}_{3}$ & 0.00 & 0.00 & 0.00 & 0.00 & 0.00 & 0.00 \\
\hline $\mathrm{H}_{2} \mathrm{~S}$ & 0.00 & 0.00 & 0.00 & 0.00 & 0.00 & 0.00 \\
\hline $\mathrm{HCN}$ & 0.00 & 0.00 & 0.00 & 0.00 & 0.00 & 0.00 \\
\hline $\mathrm{O}_{2}$ & $6,260.54$ & 0.00 & 0.00 & 0.00 & 0.00 & 0.00 \\
\hline $\cos$ & 0.00 & 0.00 & 0.00 & 0.00 & 0.00 & 0.00 \\
\hline $\mathrm{SO}_{2}$ & 0.00 & 0.00 & 0.00 & 0.00 & 0.00 & 0.00 \\
\hline Total gas flow & $45,747.31$ & 0.00 & 0.00 & $9,917.77$ & $15,668.16$ & $30,055.55$ \\
\hline $\begin{array}{l}\text { Liquids (lb.mol/h) } \\
\qquad \mathrm{H}_{2} \mathrm{O}\end{array}$ & 0.00 & $24,215.42$ & $36,215.42$ & $26,297.65$ & $20,547.25$ & $6,159.87$ \\
\hline Temperature $\left({ }^{\circ} \mathrm{F}\right)$ & 400.00 & 121.36 & 121.36 & 356.77 & 356.77 & 356.77 \\
\hline Pressure (psia) & 14.70 & 1.76 & 146.96 & 146.96 & 146.96 & 146.96 \\
\hline $\begin{array}{l}\text { Enthalpy of stream }(\mathrm{Btu} / \mathrm{h}) \\
\text { (reference, } 32^{\circ} \mathrm{F} \text { ) }\end{array}$ & $146,194,205$ & $38,996,150$ & $58,258,290$ & $368,957,384$ & $458,571,485$ & $682,784,353$ \\
\hline
\end{tabular}


TABLE 6.4 (Cont.)

\begin{tabular}{|c|c|c|c|c|c|c|c|}
\hline Stream Data & Stream 30 & Stream 31 & Stream 32 & Stream 33 & Stream 34A & Stream 34B & Stream 34C \\
\hline Description of stream & $\begin{array}{l}\text { Steam from heat } \\
\text { exchanger } 2\end{array}$ & $\begin{array}{l}\text { Steam for heating } \\
\text { feed to fuel cell }\end{array}$ & $\begin{array}{l}\text { Steam to steam } \\
\text { turbine }\end{array}$ & $\begin{array}{l}\text { Steam turbine } \\
\text { exhaust }\end{array}$ & $\begin{array}{l}\text { Water from } \\
\text { condenser }\end{array}$ & $\begin{array}{l}\text { Makeup water } \\
\text { to pump }\end{array}$ & $\begin{array}{l}\text { Wastewater for } \\
\text { treatment }\end{array}$ \\
\hline \multicolumn{8}{|l|}{ Gases (lb·mol/h) } \\
\hline $\mathrm{CO}$ & 0.00 & 0.00 & 0.00 & 0.00 & 0.00 & 0.00 & 0.00 \\
\hline $\mathrm{CO}_{2}$ & 0.00 & 0.00 & 0.00 & 0.00 & 0.00 & 0.00 & 0.00 \\
\hline $\mathrm{H}_{2}$ & 0.00 & 0.00 & 0.00 & 0.00 & 0.00 & 0.00 & 0.00 \\
\hline $\mathrm{H}_{2} \mathrm{O}$ & $36,215,42$ & $12,000.00$ & $24,215.42$ & $22,813.57$ & 0.00 & 0.00 & 0.00 \\
\hline $\mathrm{N}_{2}$ & 0.00 & 0.00 & 0.00 & 0.00 & 0.00 & 0.00 & 0.00 \\
\hline $\operatorname{Ar}$ & 0.00 & 0.00 & 0.00 & 0.00 & 0.00 & 0.00 & 0.00 \\
\hline $\mathrm{CH}_{4}$ & 0.00 & 0.00 & 0.00 & 0.00 & 0.00 & 0.00 & 0.00 \\
\hline $\mathrm{NH}_{3}$ & 0.00 & 0.00 & 0.00 & 0.00 & 0.00 & 0.00 & 0.00 \\
\hline $\mathrm{H}_{2} \mathrm{~S}$ & 0.00 & 0.00 & 0.00 & 0.00 & 0.00 & 0.00 & 0.00 \\
\hline $\mathrm{HCN}$ & 0.00 & 0.00 & 0.00 & 0.00 & 0.00 & 0.00 & 0.00 \\
\hline $\mathrm{O}_{2}$ & 0.00 & 0.00 & 0.00 & 0.00 & 0.00 & 0.00 & 0.00 \\
\hline $\cos$ & 0.00 & 0.00 & 0.00 & 0.00 & 0.00 & 0.00 & 0.00 \\
\hline $\mathrm{SO}_{2}$ & 0.00 & 0.00 & 0.00 & 0.00 & 0.00 & 0.00 & 0.00 \\
\hline Total gas flow & $36,215.42$ & $12,000.00$ & $24,215.42$ & $22,813.57$ & 0.00 & 0.00 & 0.00 \\
\hline $\begin{array}{l}\text { Liquids (lb.mol/h) } \\
\qquad \mathrm{H}_{2} \mathrm{O}\end{array}$ & 0.00 & 0.00 & 0.00 & $1,401.85$ & $13,500.17$ & $12,000.00$ & $1,500.17$ \\
\hline Temperature $\left({ }^{\circ} \mathrm{F}\right)$ & 775.00 & 775.00 & 775.00 & 121.36 & 70.00 & 70.00 & 70.00 \\
\hline Pressure (psia) & 146.96 & 146.96 & 146.96 & 1.76 & 150.00 & 150.00 & 150.00 \\
\hline $\begin{array}{l}\text { Enthalpy of stream }(\mathrm{Btu} / \mathrm{h}) \\
\text { (reference, } 32^{\circ} \mathrm{F} \text { ) }\end{array}$ & $922,758,266$ & $305,756,514$ & $617,001,752$ & $459,798,748$ & $9,234,116$ & $8,208,000$ & $1,026,116$ \\
\hline
\end{tabular}


TABLE 6.5 Descriptions of Streams of Fuel Cell System in Case 3

\begin{tabular}{ccc}
\hline $\begin{array}{c}\text { Stream and } \\
\text { Characteristics }\end{array}$ & Data & Comments on Stream Calculations \\
\hline
\end{tabular}

Stream 2B: Sulfur-free gas from

$\mathrm{H}_{2} \mathrm{~S}$ section

Temperature ( ${ }^{\circ} \mathrm{F}$ )

Pressure (psia)

Flow rate $(\mathrm{lb} \cdot \mathrm{mol} / \mathrm{h})$

$\mathrm{CO}_{2}$ (mole fraction)

$\mathrm{CO}$ (mole fraction)

Stream 8A: Expanded gases from expansion turbine

Temperature $\left({ }^{\circ} \mathrm{F}\right)$

Pressure (psia)

Flow rate $(\mathrm{lb} \cdot \mathrm{mol} / \mathrm{h})$

$\mathrm{CO}_{2}$ (mole fraction)

$\mathrm{CO}$ (mole fraction)

Stream 8B: Feed to fuel cell anode

Temperature $\left({ }^{\circ} \mathrm{F}\right.$ )

Pressure (psia)

Flow rate $(\mathrm{lb} \cdot \mathrm{mol} / \mathrm{h})$

$\mathrm{CO}_{2}$ (mole fraction)

$\mathrm{CO}$ (mole fraction)

Stream 9: Fuel cell anode exhaust

Temperature $\left({ }^{\circ} \mathrm{F}\right)$

Pressure (psia)

Flow rate $(\mathrm{lb} \cdot \mathrm{mol} / \mathrm{h})$

$\mathrm{CO}_{2}$ (mole fraction)

$\mathrm{CO}$ (mole fraction)

Stream 10: $\mathrm{CO}_{2}$-rich gases from

heat exchanger 2

Temperature $\left({ }^{\circ} \mathrm{F}\right)$

Pressure (psia)

Flow rate $(\mathrm{lb} \cdot \mathrm{mol} / \mathrm{h})$

$\mathrm{CO}_{2}$ (mole fraction)

$\mathrm{CO}$ (mole fraction)
80

450

$7,698.98$

0.0347

0.5885

$-28.67$

150

$7,698.98$

0.0347

0.5885

502.23

150

$19,698.97$

0.0136

0.2300

1300

150

$25,918.72$

0.3366

0.0699

450

150

$25,918.72$

0.3366

0.0699
The synthesis gas is cleaned in two stages. Sulfur compounds are removed before the gas is fed to the fuel cell.

Sulfur-free gases are expanded through an expansion turbine for power recovery to a pressure suitable for fuel cell operation.

The expanded gases are heated by direct steam injection to temperature of $502.23^{\circ} \mathrm{F}$. Direct injection of steam will increase the conversion of $\mathrm{CO}$ and also prevent the deposition of carbon on fuel cell anode.

The composition of the gases corresponds to $100 \%$ conversion of $\mathrm{CH}_{4}$ and $60 \%$ conversion of $\mathrm{H}_{2}$ and $\mathrm{CO}$. The temperature of gases is determined by energy balance.

The hot anode exhaust gases are cooled to a temperature of $450^{\circ} \mathrm{F}$ in heat exchanger 2 to raise high steam for bottoming cycle. 
TABLE 6.5 (Cont.)

\begin{tabular}{|c|}
\hline $\begin{array}{l}\text { Stream and } \\
\text { Characteristics }\end{array}$ \\
\hline $\begin{array}{l}\text { Stream } 11: \mathrm{CO}_{2} \text {-rich gases from } \\
\text { heat exchanger } 5 \\
\text { Temperature }\left({ }^{\circ} \mathrm{F}\right) \\
\text { Pressure }(\mathrm{psia}) \\
\text { Flow rate }(\mathrm{lb} \cdot \mathrm{mol} / \mathrm{h}) \\
\mathrm{CO}_{2} \text { (mole fraction) } \\
\mathrm{CO} \text { (mole fraction) }\end{array}$ \\
\hline $\begin{array}{l}\text { Stream 12: Feed gas to } \mathrm{CO}_{2} \text { recc } \\
\text { Temperature }\left({ }^{\circ} \mathrm{F}\right) \\
\text { Pressure (psia) } \\
\text { Flow rate (lb-mol/h) } \\
\mathrm{CO}_{2} \text { (mole fraction) } \\
\mathrm{CO} \text { (mole fraction) }\end{array}$ \\
\hline $\begin{array}{l}\text { Stream 13: } \mathrm{CO}_{2} \text {-lean gases fror } \\
\mathrm{CO}_{2} \text { recovery section } \\
\text { Temperature }\left({ }^{\circ} \mathrm{F}\right) \\
\text { Pressure (psia) } \\
\text { Flow rate }(\mathrm{lb} \cdot \mathrm{mol} / \mathrm{h}) \\
\mathrm{CO}_{2} \text { (mole fraction) } \\
\mathrm{CO} \text { (mole fraction) }\end{array}$ \\
\hline $\begin{array}{l}\text { Stream 14: } \mathrm{CO}_{2} \text {-lean gases from } \\
\text { heat exchanger } 1 \\
\text { Temperature }\left({ }^{\circ} \mathrm{F}\right) \\
\text { Pressure }(\mathrm{psia}) \\
\text { Flow rate }(\mathrm{lb} \cdot \mathrm{mol} / \mathrm{h}) \\
\mathrm{CO}_{2} \text { (mole fraction) } \\
\mathrm{CO} \text { (mole fraction) }\end{array}$ \\
\hline $\begin{array}{l}\text { Stream 15: Air to air compressor } \\
\text { Temperature }\left({ }^{\circ} \mathrm{F}\right) \\
\text { Pressure }(\mathrm{psia}) \\
\text { Flow rate }(\mathrm{lb} \cdot \mathrm{mol} / \mathrm{h}) \\
\mathrm{CO}_{2} \text { (mole fraction) } \\
\mathrm{CO} \text { (mole fraction) }\end{array}$ \\
\hline
\end{tabular}

56.14

145

$7,516.76$

0.5223

0.2387

600

145

$7,516.76$

0.5223

0.2387

81

14.7

56,568

0.0000

0.0000
The anode exhaust gases are further cooled in heat exchanger 5 to a temperature of $150^{\circ} \mathrm{F}$. The heat is utilized for preheating water for steam cycle. The amount of water vapor in the gases corresponds to the water's vapor pressure.

$\mathrm{CO}_{2}$-rich gases are cooled in a condenser to knock out the water vapor from the gases.

Fuel cell cathode takes $\mathrm{CO}_{2}$ as its feed; therefore, the $\mathrm{CO}_{2}$-lean gases along with unconverted $\mathrm{CO}$ and $\mathrm{H}_{2}$ are fed back to the fuel cell system.

The $\mathrm{CO}_{2}$-lean gases from $\mathrm{CO}_{2}$ recovery section are heated with part of the cathode exhaust gases to a temperature of $600^{\circ} \mathrm{F}$.

The cathode reaction involves both $\mathrm{O}_{2}$ and $\mathrm{CO}_{2}$. The $\mathrm{O}_{2}$ is supplied by air. Also air is supplied to burn unconverted $\mathrm{CO}$ and $\mathrm{H}_{2}$. 
TABLE 6.5 (Cont.)

\section{Stream and}

Characteristics

Data

Comments on Stream Calculations

Stream 16: Compressed air from air compressor

Temperature $\left({ }^{\circ} \mathrm{F}\right)$

Pressure (psia)

Flow rate $(\mathrm{lb} \cdot \mathrm{mol} / \mathrm{h})$

$\mathrm{CO}_{2}$ (mole fraction)

$\mathrm{CO}$ (mole fraction)

Stream 17: Gases from combustion chamber

Temperature $\left({ }^{\circ} \mathrm{F}\right)$

Pressure (psia)

Flow rate $(\mathrm{lb} \cdot \mathrm{mol} / \mathrm{h})$

$\mathrm{CO}_{2}$ (mole fraction)

$\mathrm{CO}$ (mole fraction)

Stream 18: Fuel cell cathode feed

Temperature $\left({ }^{\circ} \mathrm{F}\right)$

Pressure (psia)

Flow rate $(\mathrm{lb} \cdot \mathrm{mol} / \mathrm{h})$

$\mathrm{CO}_{2}$ (mole fraction)

$\mathrm{CO}$ (mole fraction)

Stream 19: Fuel cell cathode exhaust

Temperature $\left({ }^{\circ} \mathrm{F}\right)$

Pressure (psia)

Flow rate $(\mathrm{lb} \cdot \mathrm{mol} / \mathrm{h})$

$\mathrm{CO}_{2}$ (mole fraction)

$\mathrm{CO}$ (mole fraction)

Stream 20: Cathode exhaust from expansion turbine

Temperature $\left({ }^{\circ} \mathrm{F}\right)$

Pressure (psia)

Flow rate $(\mathrm{lb} \cdot \mathrm{mol} / \mathrm{h})$

$\mathrm{CO}_{2}$ (mole fraction)

$\mathrm{CO}$ (mole fraction)
$54,455.70$

0.0085

0.0000
667.23

14.70

713.05 The air is compressed to the operating

150

56,568

0.0000

0.0000

$1,411.87$

150

$62,343.64$

0.0918

0.0000

980.33

150

$62,343.64$

0.0918

0.0000

1,300

150

$54,455.70$

0.0085

0.0000

Part of the $\mathrm{CO}_{2}$ in the cathode feed is consumed by cathode reaction. The temperature of gases is by energy balance.

High-temperature cathode exhaust gases are expanded in expansion turbine to recover power.
The composition of gases is based on the composition of gases from $\mathrm{CO}_{2}$ recovery and air from compressor. The temperature is adiabatic temperature.

The gases from the combustion chamber are cooled to a suitable temperature of the fuel cell in heat exchanger 3. 
TABLE 6.5 (Cont.)

\begin{tabular}{|c|c|c|}
\hline $\begin{array}{l}\text { Stream and } \\
\text { Characteristics }\end{array}$ & Data & Comments on Stream Calculations \\
\hline \multicolumn{3}{|l|}{ Stream 21: Cathode exhaust } \\
\hline Temperature $\left({ }^{\circ} \mathrm{F}\right)$ & 667.23 & The gases from the expansion turbine are \\
\hline Pressure (psia) & 14.70 & at $667^{\circ} \mathrm{F}$. Part of this gas stream \\
\hline Flow rate $(\mathrm{lb} \cdot \mathrm{mol} / \mathrm{h})$ & $8,708.38$ & is used in heating the gases from the $\mathrm{CO}_{2}$ \\
\hline $\mathrm{CO}_{2}$ (mole fraction) & 0.0085 & recovery system. \\
\hline CO (mole fraction) & 0.0000 & \\
\hline \multicolumn{3}{|l|}{ Stream 22: Exhaust to stack } \\
\hline Temperature $\left({ }^{\circ} \mathrm{F}\right)$ & 100 & -...- \\
\hline Pressure (psia) & 14.70 & \\
\hline Flow rate $(\mathrm{lb} \cdot \mathrm{mol} / \mathrm{h})$ & $8,708.38$ & \\
\hline $\mathrm{CO}_{2}$ (mole fraction) & 0.0085 & \\
\hline $\mathrm{CO}$ (mole fraction) & 0.0000 & \\
\hline \multicolumn{3}{|l|}{ Stream 23: Cathode exhaust } \\
\hline Temperature $\left({ }^{\circ} \mathrm{F}\right)$ & 667.23 & The second portion of the cathode exhaust \\
\hline Pressure (psia) & 14.70 & is utilized in raising the temperature of \\
\hline Flow rate $(\mathrm{lb} \cdot \mathrm{mol} / \mathrm{h})$ & $45,747.31$ & water for the steam cycle. \\
\hline $\mathrm{CO}_{2}$ (mole fraction) & 0.0085 & \\
\hline $\mathrm{CO}$ (mole fraction) & 0.0000 & \\
\hline \multicolumn{3}{|l|}{ Stream 24: Exhaust to stack } \\
\hline Temperature $\left({ }^{\circ} \mathrm{F}\right)$ & 400 & $-\cdots-$ \\
\hline Pressure (psia) & 14.70 & \\
\hline Flow rate $(\mathrm{lb} \cdot \mathrm{mol} / \mathrm{h})$ & $45,747.31$ & \\
\hline $\mathrm{CO}_{2}$ (mole fraction) & 0.0085 & \\
\hline $\mathrm{CO}$ (mole fraction) & 0.0000 & \\
\hline \multicolumn{3}{|l|}{$\begin{array}{l}\text { Stream 25: Water from steam } \\
\text { condenser }\end{array}$} \\
\hline Temperature $\left({ }^{\circ} \mathrm{F}\right)$ & 121.36 & Water from the steam condenser is for steam \\
\hline Pressure (psia) & 1.76 & cycle. \\
\hline Flow rate $(\mathrm{lb} \cdot \mathrm{mol} / \mathrm{h})$ & $38,996,150$ & \\
\hline Quality & 0 & \\
\hline \multicolumn{3}{|l|}{ Stream 26: Water from pump } \\
\hline Temperature $\left({ }^{\circ} \mathrm{F}\right)$ & 121.36 & Water from the pump is for steam cycle. \\
\hline Pressure (psia) & 146.96 & \\
\hline Flow rate $(\mathrm{lb} \cdot \mathrm{mol} / \mathrm{h})$ & $38,996,150$ & \\
\hline Quality & 0 & \\
\hline
\end{tabular}




\section{Stream and \\ Characteristics \\ Stream 27: Steam from \\ heat exchanger 5 \\ Temperature $\left({ }^{\circ} \mathrm{F}\right)$ \\ Pressure (psia) \\ Flow rate $(\mathrm{lb} \cdot \mathrm{mol} / \mathrm{h})$ \\ Quality}

Stream 28: Steam from

heat exchanger 4

Temperature $\left({ }^{\circ} \mathrm{F}\right)$

Pressure (psia)

Flow rate $(\mathrm{lb} \cdot \mathrm{mol} / \mathrm{h})$

Quality

Stream 29: Steam from

heat exchanger 3

Temperature $\left({ }^{\circ} \mathrm{F}\right)$

Pressure (psia)

Flow rate $(\mathrm{lb} \cdot \mathrm{mol} / \mathrm{h})$

Quality

356.77

146.96

$38,996,150$

0.8299

Stream 30: Steam from heat exchanger 2

Temperature $\left({ }^{\circ} \mathrm{F}\right)$

Pressure (psia)

Flow rate $(\mathrm{lb} \cdot \mathrm{mol} / \mathrm{h})$

Quality

Stream 31: Steam for heating

fuel cell feed

Temperature $\left({ }^{\circ} \mathrm{F}\right)$

Pressure (psia)

Flow rate $(\mathrm{lb} \cdot \mathrm{mol} / \mathrm{h})$

Quality

Stream 32: Superheated steam

to steam turbine

Temperature ( $\left.{ }^{\circ} \mathrm{F}\right)$

Pressure (psia)

Flow rate $(\mathrm{lb} \cdot \mathrm{mol} / \mathrm{h})$

Quality

356.77

146.96

$38,996,150$

0.2738

356.77

146.96

$38,996,150$

0.4326
775

146.96

$38,996,150$

1

775

146.96

12,000

1

775

146.96

$4,215.42$

Steam is from heat exchanger 5.

Steam is from heat exchanger 4.

Steam is from heat exchanger 3.

Comments on Stream Calculations 
TABLE 6.5 (Cont.)

\begin{tabular}{|c|c|c|c|}
\hline $\begin{array}{l}\text { Stream and } \\
\text { Characteristics }\end{array}$ & Data & & Comments on Stream Calculations \\
\hline \multicolumn{4}{|c|}{$\begin{array}{l}\text { Stream 33: Expanded steam from } \\
\text { steam turbine }\end{array}$} \\
\hline Temperature $\left({ }^{\circ} \mathrm{F}\right)$ & 121.36 & $-\cdots$ & \\
\hline Pressure (psia) & 1.76 & & \\
\hline Flow rate $(\mathrm{lb} \cdot \mathrm{mol} / \mathrm{h})$ & $24,215.42$ & & \\
\hline Quality & 0.9421 & & \\
\hline \multicolumn{4}{|l|}{$\begin{array}{l}\text { Stream } 34 \mathrm{~A} \text { : Condensate from } \\
\text { anode exhaust condenser }\end{array}$} \\
\hline Temperature $\left({ }^{\circ} \mathrm{F}\right)$ & 70 & -..-- & \\
\hline Pressure (psia) & 150 & & \\
\hline Flow rate $(\mathrm{lb} \cdot \mathrm{mol} / \mathrm{h})$ & $13,500.17$ & & \\
\hline Quality & 0 & & \\
\hline \multicolumn{4}{|l|}{$\begin{array}{l}\text { Stream 34B: Makeup water to } \\
\text { steam cycle pump }\end{array}$} \\
\hline Temperature $\left({ }^{\circ} \mathrm{F}\right)$ & 70 & ---- & \\
\hline Pressure (psia) & 150 & & \\
\hline Flow rate $(\mathrm{lb} \cdot \mathrm{mol} / \mathrm{h})$ & 12,000 & & \\
\hline Quality & 0 & & \\
\hline \multicolumn{4}{|l|}{$\begin{array}{l}\text { Stream } 34 \mathrm{C} \text { : Wastewater for } \\
\text { treatment }\end{array}$} \\
\hline Temperature $\left({ }^{\circ} \mathrm{F}\right)$ & 70 & -..-- & \\
\hline Pressure (psia) & 150 & & \\
\hline Flow rate $(\mathrm{lb} \cdot \mathrm{mol} / \mathrm{h})$ & $1,500.17$ & & \\
\hline Quality & 0 & & \\
\hline
\end{tabular}




\subsection{Glycol Process for $\mathrm{CO}_{2}$ Recovery}

Figure 6.4 is an overall flow diagram of a glycol-based $\mathrm{CO}_{2}$ recovery system. It is similar to the glycol system described in Section 4. In this system, the $\mathrm{CO}_{2}$ is absorbed under pressure in a low-temperature glycol absorber. The pressure is released through a hydraulic turbine and in a series of flash tanks. The first tank in that series, the slump tank, allows for recovery of hydrogen from the rich absorbent. The subsequent tanks release $\mathrm{CO}_{2}$ for disposal. The use of a series of tanks reduces the compression requirement. Table 6.6 is a line list corresponding to Figure 6.4. Stream descriptions and associated assumptions are provided in Table 6.7.

\subsection{Fuel Cell, Steam Cycle, and Plant Performance}

Use of the fuel cell topping cycle with methanol-based $\mathrm{H}_{2} \mathrm{~S}$ recovery and glycol-based $\mathrm{CO}_{2}$ recovery results in a net plant output of $340 \mathrm{MW}, 18 \%$ less than in the base case plant without $\mathrm{CO}_{2}$ recovery. Table 6.8 lists the topping cycle output, steam cycle output, and internal plant consumption for the base case (no $\mathrm{CO}_{2}$ recovery) and for the current case, Case 3 . The most significant losses are the consumption of power for $\mathrm{CO}_{2}$ compression and reduced steam cycle output.

\subsection{Economics}

Details of the capital investment estimates for the $\mathrm{H}_{2} \mathrm{~S}$ recovery system, the fuel cell system, and the $\mathrm{CO}_{2}$ recovery system are presented in Tables 6.9, 6.10, and 6.11, respectively. A summary of capital costs, including indirect capital investment, operating, and maintenance costs, is provided in Section 9. 


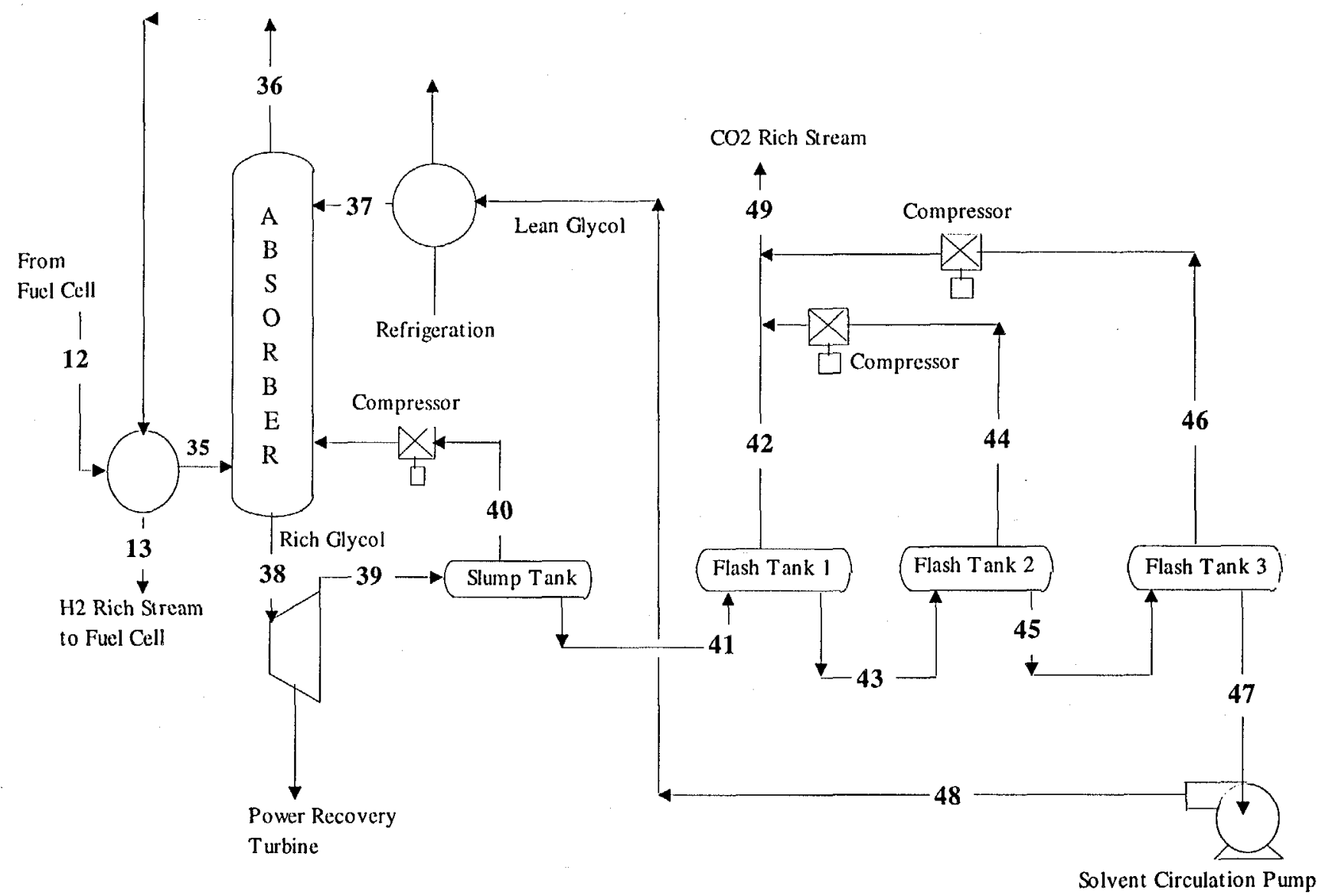

FIGURE 6.4 Flow Diagram of Glycol Process for $\mathrm{CO}_{2}$ Recovery and Chilled Methanol Process for $\mathrm{H}_{2} \mathrm{~S}$ Recovery with Fuel Cell Topping Cycle in Case 3 
TABLE 6.6 Stream Flows of Glycol System for $\mathrm{CO}_{2}$ Removal in Case 3

\begin{tabular}{|c|c|c|c|c|c|c|}
\hline Stream Data & Stream 12 & Stream 13 & Stream 35 & Stream 36 & Stream 37 & Stream 38 \\
\hline Description of stream & $\begin{array}{l}\text { Feed gas from } \\
\text { fuel cell system }\end{array}$ & $\begin{array}{l}\mathrm{H}_{2} \text {-rich gas after } \\
\text { heat exchanger }\end{array}$ & Absorber feed & $\begin{array}{l}\text { Clean fuel gas } \\
\text { from absorber }\end{array}$ & $\begin{array}{l}\text { Leani giycol solvent } \\
\text { to absorber }\end{array}$ & $\begin{array}{l}\text { Rich giycol } \\
\text { from absorber }\end{array}$ \\
\hline \multicolumn{7}{|l|}{ Gases (lb·mol/h) } \\
\hline $\mathrm{CO}$ & $1,812.16$ & $1,794.14$ & $1,812.16$ & $1,794.14$ & 0.00 & 181.23 \\
\hline \multirow{2}{*}{$\mathrm{CO}_{2}$} & $8,724.66$ & $3,926.10$ & $8,724.66$ & $3,926.10$ & 210.13 & $5,110.91$ \\
\hline & $1,693.50$ & $1,688.08$ & $1,693.50$ & $1,688.08$ & 10.41 & 158.18 \\
\hline $\mathrm{H}_{2} \mathrm{O}$ & 78.96 & 0.00 & 78.96 & 0.00 & 0.00 & 79.76 \\
\hline $\mathrm{N}_{2}^{2}$ & 36.44 & 35.71 & 36.44 & 35.71 & 0.00 & 7.29 \\
\hline $\operatorname{Ar}$ & 72.73 & 72.73 & 72.73 & 72.73 & 0.00 & 0.00 \\
\hline $\mathrm{CH}_{4}$ & 0.00 & 0.00 & 0.00 & 0.00 & 0.00 & 0.00 \\
\hline $\mathrm{NH}_{3}$ & 0.00 & 0.00 & 0.00 & 0.00 & 0.00 & 0.00 \\
\hline $\mathrm{H}_{2} \mathrm{~S}$ & 0.01 & 0.00 & 0.01 & 0.00 & 0.00 & 0.01 \\
\hline $\mathrm{HCN}$ & 0.00 & 0.00 & 0.00 & 0.00 & 0.00 & 0.00 \\
\hline $\mathrm{O}_{2}$ & 0.00 & 0.00 & 0.00 & 0.00 & 0.00 & 0.00 \\
\hline $\cos$ & 0.00 & 0.00 & 0.00 & 0.00 & 0.00 & 0.00 \\
\hline $\mathrm{SO}_{2}$ & 0.00 & 0.00 & 0.00 & 0.00 & 0.00 & 0.00 \\
\hline Total gas flow & $12,418.46$ & $7,516.76$ & $12,418.46$ & $7,516.76$ & 220.54 & $5,537.38$ \\
\hline \multicolumn{7}{|l|}{ Glycol solvent } \\
\hline Temperature $\left({ }^{\circ} \mathrm{F}\right)$ & 70.00 & 56.14 & 55.00 & 30.00 & 30.00 & 50.36 \\
\hline Pressure (psia) & 150.00 & 145.00 & 150.00 & 145.00 & 150.00 & 145.00 \\
\hline $\begin{array}{l}\text { Enthalpy of stream }(\mathrm{Btu} / \mathrm{h}) \\
\text { (reference, } 32^{\circ} \mathrm{F} \text { ) }\end{array}$ & $3,933,489$ & $1,441,041$ & $2,373,591$ & $-119,000$ & $-5,712,163$ & $53,306,378$ \\
\hline
\end{tabular}


TABLE 6.6 (Cont.)

\begin{tabular}{|c|c|c|c|c|c|c|}
\hline Stream Data & Stream 39 & Stream 40 & Stream 41 & Stream 42 & Stream 43 & Stream 44 \\
\hline Description of stream & $\begin{array}{l}\text { Rich glycol solvent } \\
\text { after turbine } 1\end{array}$ & $\begin{array}{l}\text { Gases from } \\
\text { slump tank }\end{array}$ & $\begin{array}{l}\text { Rich glycol to } \\
\text { flash tank } 1\end{array}$ & $\begin{array}{l}\mathrm{CO}_{2} \text {-rich gas from } \\
\text { flash tank } 1\end{array}$ & $\begin{array}{l}\text { Rich glycol to } \\
\text { flash tank } 2\end{array}$ & $\begin{array}{l}\mathrm{CO}_{2} \text {-rich gas from } \\
\text { flash tank } 2\end{array}$ \\
\hline \multicolumn{7}{|l|}{ Gases $(\mathrm{lb} \cdot \mathrm{mol} / \mathrm{h})$} \\
\hline $\mathrm{CO}$ & 181.23 & 163.10 & 18.12 & 18.12 & 0.00 & 0.00 \\
\hline $\mathrm{CO}_{2}$ & $5,110.91$ & 102.22 & $5,008.69$ & $3,756.52$ & $1,252.17$ & 876.52 \\
\hline $\mathrm{H}_{2}$ & 158.18 & 142.36 & 15.82 & 1.58 & 14.24 & 2.14 \\
\hline $\mathrm{H}_{2} \mathrm{O}$ & 79.76 & 0.80 & 78.96 & 78.96 & 0.00 & 0.00 \\
\hline $\mathrm{N}_{2}$ & 7.29 & 6.56 & 0.73 & 0.73 & 0.00 & 0.00 \\
\hline $\mathrm{Ar}$ & 0.00 & 0.00 & 0.00 & 0.00 & 0.00 & 0.00 \\
\hline $\mathrm{CH}_{4}$ & 0.00 & 0.00 & 0.00 & 0.00 & 0.00 & 0.00 \\
\hline $\mathrm{NH}_{3}$ & 0.00 & 0.00 & 0.00 & 0.00 & 0.00 & 0.00 \\
\hline $\mathrm{H}_{2} \mathrm{~S}$ & 0.01 & 0.00 & 0.01 & 0.00 & 0.01 & 0.00 \\
\hline $\mathrm{HCN}$ & 0.00 & 0.00 & 0.00 & 0.00 & 0.00 & 0.00 \\
\hline $\mathrm{O}_{2}$ & 0.00 & 0.00 & 0.00 & 0.00 & 0.00 & 0.00 \\
\hline $\cos$ & 0.00 & 0.00 & 0.00 & 0.00 & 0.00 & 0.00 \\
\hline $\mathrm{SO}_{2}$ & 0.00 & 0.00 & 0.00 & 0.00 & 0.00 & 0.00 \\
\hline Total gas flow & $5,537.38$ & 415.04 & $5,122.33$ & $3,855.91$ & $1,266.42$ & 878.66 \\
\hline $\begin{array}{l}\text { Liquids (lb·mol/h) } \\
\text { Glycol solvent }\end{array}$ & $20,802.84$ & 0.00 & $20,802.84$ & 0.00 & $20,802.84$ & 0.00 \\
\hline Temperature $\left({ }^{\circ} \mathrm{F}\right)$ & 49.97 & 49.57 & 49.57 & 34.81 & 34.81 & 31.33 \\
\hline Pressure (psia) & 50.00 & 50.00 & 50.00 & 25.00 & 25.00 & 14.70 \\
\hline $\begin{array}{l}\text { Enthalpy of stream }(B t u / h) \\
\left.\text { (reference, } 32^{\circ} \mathrm{F}\right)\end{array}$ & $52,160,172$ & 53,779 & $50,942,277$ & 95,822 & $8,065,266$ & $-5,201$ \\
\hline
\end{tabular}


TABLE 6.6 (Cont.)

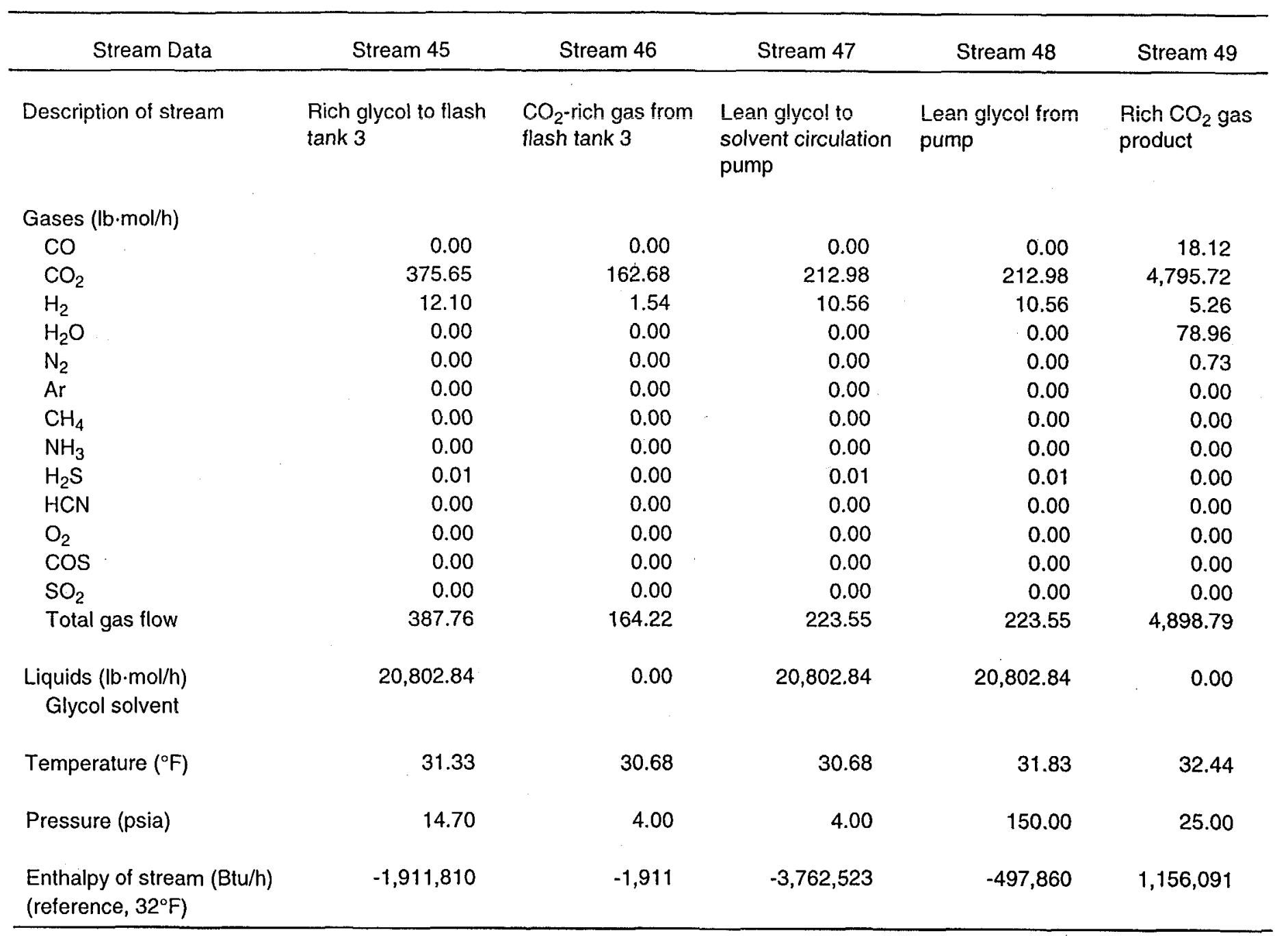


TABLE 6.7 Descriptions of Streams of Glycol Process for $\mathrm{CO}_{2}$ Removal in Case 3

\section{Stream and \\ Characteristics}

Data

Comments on Stream Calculations
Stream 12: $\mathrm{CO}_{2}$-rich gas from

fuel cell system

Temperature ( ${ }^{\circ} \mathrm{F}$ )

Pressure (psia)

Flow rate $(\mathrm{lb} \cdot \mathrm{mol} / \mathrm{h})$

$\mathrm{CO}_{2}$ (mole fraction)

Stream 35: Feed gas to absorber

Temperature ( ${ }^{\circ} \mathrm{F}$ )

Pressure (psia)

Flow rate $(\mathrm{lb} \cdot \mathrm{mol} / \mathrm{h})$

$\mathrm{CO}_{2}$ (mole fraction)

Stream 36: Fuel gas from absorber

Temperature ( $\left.{ }^{\circ} \mathrm{F}\right)$

Pressure (psia)

Flow rate $(\mathrm{lb} \cdot \mathrm{mol} / \mathrm{h})$

$\mathrm{CO}_{2}$ (mole fraction)

Stream 13: Fuel gas after

heat exchanger

Temperature $\left({ }^{\circ} \mathrm{F}\right)$

Pressure (psia)

Flow rate $(\mathrm{lb} \cdot \mathrm{mol} / \mathrm{h})$

$\mathrm{CO}_{2}$ (mole fraction)

Stream 37: Lean glycol to the of absorber

Temperature ( ${ }^{\circ} \mathrm{F}$ )

Pressure (psia)

Flow rate $(\mathrm{lb} \cdot \mathrm{mol} / \mathrm{h})$

$\mathrm{CO}_{2}$ (mole fraction)

70 The synthesis gas is cleaned in two stages.

150 First, sulfur compounds are removed

$12,418.46$ with chilled methanol. Then they are fed

0.7026 to another absorption column for $\mathrm{CO}_{2}$ recovery.

The $\mathrm{CO}_{2}$-rich gas is cooled against

150 the cold fuel gas from the top of the

$12,418.46$ absorber to a temperature of $55^{\circ} \mathrm{F}$.

30

145

$7,516.76$

0.5223

56.14

145

$7,516.76$

0.5223

30

150

$21,023.38$

0.01
The composition of this stream corresponds to a $\mathrm{CO}_{2}$-removal efficiency of $55 \%$. Also, other gases like $\mathrm{H}_{2} \mathrm{~S}, \mathrm{COS}$, and $\mathrm{H}_{2}$ are absorbed by the solvent. The temperature of this stream is close to the temperature of lean solvent entering the absorber at the top.

Fuel gas is heated against the $\mathrm{CO}_{2}$ rich gases from the fuel cell section.

Lean glycol solvent contains residual $\mathrm{CO}_{2}$. $50 \%$ excess solvent is used. The solvent is cooled to $30^{\circ} \mathrm{F}$ by refrigeration. 
TABLE 6.7 (Cont.)

Stream and

Characteristics

Stream 38: Rich glycol solvent

from absorber

Temperature $\left({ }^{\circ} \mathrm{F}\right)$

Pressure (psia)

Flow rate $(\mathrm{lb} \cdot \mathrm{mol} / \mathrm{h})$

$\mathrm{CO}_{2}$ (mole fraction)

Stream 39: Rich glycol solvent from turbine 1

Temperature $\left({ }^{\circ} \mathrm{F}\right)$

Pressure (psia)

Flow rate $(\mathrm{lb} \cdot \mathrm{mol} / \mathrm{h})$

$\mathrm{CO}_{2}$ (mole fraction)

Stream 40: Flash gas

Temperature $\left({ }^{\circ} \mathrm{F}\right)$

Pressure (psia)

Flow rate $(\mathrm{lb} \cdot \mathrm{mol} / \mathrm{h})$

$\mathrm{CO}_{2}$ (mole fraction)

Stream 41: Rich glycol to

high-pressure flash tank 1

Temperature ( ${ }^{\circ} \mathrm{F}$ )

Pressure (psia)

Flow rate $(\mathrm{lb} \cdot \mathrm{mol} / \mathrm{h})$

$\mathrm{CO}_{2}$ (mole fraction)

Stream 42: $\mathrm{CO}_{2}$-rich flash gas from

high-pressure flash tank

Temperature $\left({ }^{\circ} \mathrm{F}\right)$

Pressure (psia)

Flow rate $(\mathrm{lb} \cdot \mathrm{mol} / \mathrm{h})$

$\mathrm{CO}_{2}$ (mole fraction)
In first stage, the gases are flashed to

50.36 Flow rate reflects lean glycol solvent plus

145 absorbed $\mathrm{CO}_{2}, \mathrm{H}_{2} \mathrm{~S}$, and other gases. The

$26,340.22$

0.1940

temperature increases because of the heat of absorption of $\mathrm{CO}_{2}$ and $\mathrm{H}_{2} \mathrm{~S}$.

49.97 This stream is the exit stream from power

50 recovery turbine. Exit pressure has

$26,340.22$ been selected to avoid release of $\mathrm{CO}_{2}$

0.1940 and $\mathrm{H}_{2} \mathrm{~S}$ while allowing some recovery of work of pressurization. The change in temperature over the turbine is estimated from change in enthalpy, which is taken to be equal to flow work.

49.57 $\mathrm{CO}_{2}$ and $\mathrm{H}_{2} \mathrm{~S}$ are released from the glycol solvent in the slump tank. This 415.04 stream is compressed and recycled to the
0.2463 absorber to decrease the losses of valuable gases like $\mathrm{H}_{2}$ and $\mathrm{CO}$.

49.57 The $\mathrm{CO}_{2}$ from the rich glycol solvent is 50 released in stages. a pressure of 25 psia. The amount of $\mathrm{CO}_{2}$ remaining in the solvent 0.9742 depends on pressure, and the $\mathrm{CO}_{2}$ released is calculated by mass balance. 
TABLE 6.7 (Cont.)

Stream and
Characteristics

Stream 45: Glycol solvent from

intermediate-pressure flash tank

Temperature ( ${ }^{\circ} \mathrm{F}$ )

Pressure (psia)

31.33

31.33

14.70

The amount of $\mathrm{CO}_{2}$ in solvent and released

878.66

0.9976 as gas is calculated as in stream 42 . Sufficient residence is provided for the gases to separate from solvent. This determines tank volume.

Flow rate $(\mathrm{lb} \cdot \mathrm{mol} / \mathrm{h})$

$\mathrm{CO}_{2}$ (mole fraction)

Stream 46: $\mathrm{CO}_{2}$-rich flash gas from low-pressure flash tank

Temperature $\left({ }^{\circ} \mathrm{F}\right)$

Pressure (psia)

Flow rate $(\mathrm{lb} \cdot \mathrm{mol} / \mathrm{h})$

$\mathrm{CO}_{2}$ (mole fraction)
30.68 Glycol solvent is flashed to a pressure of
4.04 psia to remove as much $\mathrm{CO}_{2}$ as
164.22 possible. The lower residual amount of
$0.9906 \mathrm{CO}_{2}$ in lean glycol solvent reduces the circulation rate of solvent.

Stream 47: Lean glycol solvent from low-pressure flash tank

Temperature $\left({ }^{\circ} \mathrm{F}\right)$

Pressure (psia)

30.68

Flow rate $(\mathrm{lb} \cdot \mathrm{mol} / \mathrm{h})$

$\mathrm{CO}_{2}$ (mole fraction)

4.0

$21,026.38$

.0101 
TABLE 6.7 (Cont.)

Stream and

Characteristics

Data

Comments on Stream Calculations

Stream 48: Lean glycol solvent after circulation pump

Temperature $\left({ }^{\circ} \mathrm{F}\right)$

Pressure (psia)

Flow rate $(\mathrm{lb} \cdot \mathrm{mol} / \mathrm{h})$

$\mathrm{CO}_{2}$ (mole fraction)

31.83 The lean solvent is pressurized to the

150 absorber operating pressure by using a pump.

$21,026.38$ The change in temperature results from work

0.0101 of compression. The solvent is chilled before being sent to the absorber.

Stream 49: $\mathrm{CO}_{2}$-rich product gas

Temperature $\left({ }^{\circ} \mathrm{F}\right)$

Pressure (psia)

32.44 Flash gases from intermediate- and low-

Flow rate $(\mathrm{lb} \cdot \mathrm{mol} / \mathrm{h})$

25.0 pressure flash tanks are compressed to the $\mathrm{CO}_{2}$ (mole fraction) pressure of stream 42 . Streams 42 ,

0.979044 , and 46 are combined for further compression for pipeline. 
TABLE 6.8 Power Output, Plant Power Use, and Net Power Output for Base Case and Case 3 Fuel Cell/ Glycol Process

\begin{tabular}{lcc}
\hline & \multicolumn{2}{c}{ Power (MW) } \\
\cline { 2 - 3 } Power Variable & Base Case & Fuel Cell Case \\
\hline Power output & & \\
Gas turbine or fuel cell & 298.8 & 246.7 \\
Steam turbine & 159.4 & 171.8 \\
& & \\
Internal power consumption & & \\
CO $_{2}$ recovery & & \\
CO & & \\
Solvent compression & 0 & -24.9 \\
Solvent refrigeration & 0 & -2.9 \\
$\quad$ Others & 0 & -1.3 \\
Gasification system & & \\
& 0.4 & -48.9 \\
Net power output & -44.7 & 340.1 \\
Energy penalty & & 73.4 \\
\hline
\end{tabular}

a Includes $\mathrm{H}_{2} \mathrm{~S}$ recovery system energy use. 
TABLE 6.9 Sizing and Cost Estimation for Major Equipment Used for $\mathrm{H}_{2} \mathrm{~S}$ Removal in Chilled Methanol Process in Case 3

1. Gas-Gas Heat Exchanger for Raw Gas Cooling

a) with $\mathrm{H}_{2} \mathrm{~S}$-Rich Gas

$\mathrm{Q}=\operatorname{Load}(\mathrm{Btu} / \mathrm{h})$

Tha $=$ Inlet temperature of hot fluid $\left({ }^{\circ} \mathrm{F}\right)$

$\mathrm{Thb}=$ Outlet temperature of hot fluid ( $\left.{ }^{\circ} \mathrm{F}\right)$

Pressure of hot gases (psia)

$\mathrm{Tca}=$ Inlet temperature of cold fluid $\left({ }^{\circ} \mathrm{F}\right)$

$\mathrm{Tcb}=$ Outlet temperature of cold fluid $\left({ }^{\circ} \mathrm{F}\right)$

Delta T1

Delta T2

103,571

104.55

$-10$

456

$-29.9$

84.50

20.045

Log mean temperature difference ( ${ }^{\circ} \mathrm{F}$ )

20

20

Overall heat transfer coefficient $\left(\mathrm{Btu} / \mathrm{h} / \mathrm{ft}^{2} /{ }^{\circ} \mathrm{F}\right)$

5

Heat transfer area $\left(\mathrm{ft}^{2}\right)$

Operating pressure (psia)

1,038

275

Pressure factor

1.165

Materials correction factor

1

Module factor

3.2

(includes all of the supporting equipment and connections and installation)

Purchased cost of heat exchanger in 1987

(mild steel construction, shell and tube floating head)

$C E$ index for process equipment in 1987

CE index for process equipment in 1995

Installed cost of heat exchanger in 1995

$\$ 23,000$

373.9

b) with $\mathrm{H}_{2} \mathrm{~S}$-Lean Fuel Gas

$Q=\operatorname{Load}(B t u / h)$

Tha $=$ Inlet temperature of hot fluid $\left({ }^{\circ} \mathrm{F}\right)$

$\mathrm{Thb}=$ Outlet temperature of hot fluid $\left({ }^{\circ} \mathrm{F}\right)$

Pressure of hot gases (psia)

$\mathrm{Tca}=$ Inlet temperature of cold fluid $\left({ }^{\circ} \mathrm{F}\right)$

$\mathrm{Tcb}=$ Outlet temperature of cold fluid $\left({ }^{\circ} \mathrm{F}\right)$

Delta $\mathrm{T} 1$

Delta T2

Log mean temperature difference $\left({ }^{\circ} \mathrm{F}\right)$

Overall heat transfer coefficient $\left(\mathrm{Btu} / \mathrm{h} / \mathrm{ft} \mathrm{t}^{2} /{ }^{\circ} \mathrm{F}\right)$

Heat transfer area $\left(\mathrm{ft}^{2}\right)$

Operating pressure (psia)

Pressure factor

Materials correction factor

Module factor

(includes all of the supporting equipment and connections and

installation)

Purchased cost of heat exchanger in 1987

(mild steel construction, shell and tube floating head)

CE index for process equipment in 1987

CE index for process equipment in 1995

Installed cost of heat exchanger in 1995

$8,036,992$

104.55

$-34$

456

$-70$

80

24.545

36

30

53,888

275

1.165

1

3.2

$\$ 350,000$

320

373.9
$\$ 100,187$

\section{(1)}

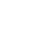




\section{2. $\mathrm{H}_{2} \mathrm{~S}$ Absorption Column}

Diameter of tower $(\mathrm{ft}) \quad 7$

HETP (ft) 3

Number of theoretical stages $\quad 15$

Absorber tower height (ft) $\quad 49$

( $4 \mathrm{ft}$ for inlet, outlet and gas, and liquid distributions)

Volume of packing $\left(\mathrm{ft}^{3}\right)$

1,733

Pressure factor

Cost per foot of column height $\quad \$ 950$

(mild steel construction)

Materials correction factor

Module factor

CE index for process equipment in 1987

CE index for process equipment in 1995

373.9

Installed cost of absorber in 1995

$\$ 588,291$

Cost of packing per cubic foot

( 2 in. pall rings-metal)

$\$ 63.5$

Total cost of packing

$\$ 110,014$

3. $\mathrm{H}_{2} \mathrm{~S}$ Stripping Column

Diameter of tower $(\mathrm{ft})$

HETP ( $\mathrm{ft})$

Number of theoretical stages

Absorber tower height ( $\mathrm{ft}$ )

( $4 \mathrm{ft}$ for inlet, outlet and gas, and liquid distributions)

Volume of packing $\left(\mathrm{ft}^{3}\right)$

Pressure factor

Cost per $\mathrm{ft}$ of column height

(mild steel construction)

Module factor

CE index for process equipment in 1987

CE index for process equipment in 1995

Installed cost of absorber in 1995

373.9

Cost of packing per cubic foot

(2-in. pall rings-metal)

Total cost of packing 
TABLE 6.9 (Cont.)

4. Flash Drum 1

Methanol flow rate $(\mathrm{lb} / \mathrm{h})$

123,450

Density of methanol (lb/gal)

6.55

Residence time (s)

180

Slump tank volume (gal)

942

Pressure factor

Module factor

2.08

Purchased cost of flash drum in 1987

$\$ 5,200$

(mild steel construction)

CE index for process equipment in 1987

320

CE index for process equipment in 1995

373.9

Installed cost of flash drum in 1995

$\$ 12,638$

5. Recycle Compressor

Inlet pressure (psia)

300

456

Outlet pressure (psia)

72

Compressor size (hp)

$\$ 32,000$

Purchased cost of centrifugal compressor in 1987

(includes electric motor drive and gear reducer)

Size factor

Module factor

CE index for process equipment in 1987

CE index for process equipment in 1995

373.9

Installed cost of compressor in 1995

$\$ 97,214$

6. Flash Drum 2

Methanol flow rate $(\mathrm{lb} / \mathrm{h})$

123,450

6.55

Density of methanol (lb/gal)

180

Slump tank volume (gal)

942

Pressure factor

1

Module factor

2.08

Purchased cost of flash drum in 1987

$\$ 5,200$

(mild steel construction)

CE index for process equipment in 1987

320

CE index for process equipment in 1995

373.9

Installed cost of flash drum in 1995 
7. Flash Drum 3

Methanol flow rate $(\mathrm{lb} / \mathrm{h})$

123,450

Density of methanol (lb/gal)

6.55

Residence time ( $s$ )

180

Slump tank volume (gal)

942

Pressure factor

1

Module factor

2.08

Purchased cost of flash drum in 1987

$\$ 5,200$

(mild steel construction)

CE index for process equipment in 1987

320

CE index for process equipment in 1995

373.9

Installed cost of flash drum in 1995

$\$ 12,638$

8. Flash Gas Compressor 1

Inlet pressure (psia)

20.00

150.00

Outlet pressure (psia)

57

Compressor size (hp)

$\$ 27,000$

(includes electric motor drive and gear reducer)

Size factor

Module factor

CE index for process equipment in 1987

320

CE index for process equipment in 1995

373.9

Installed cost of compressor in 1995

$\$ 82,024$

9. Flash Gas Compressor 2

Inlet pressure (psia)

Outlet pressure (psia)

14.70

150.00

Compressor size (hp)

154

Purchased cost of centrifugal compressor in 1987

$\$ 60,000$

(includes electric motor drive and gear reducer)

Size factor

Module factor

CE index for process equipment in 1987

320

CE index for process equipment in 1995

373.9

Installed cost of compressor in 1995

$\$ 182,276$

10. Solvent Circulation Pump

Horse power

115

Size exponent

(includes motor, coupling, base; cast iron, horizontal) Module factor

CE index for process equipment in 1987

320

CE index for process equipment in 1995

373.9

Installed cost of solvent pump in 1995 
11. Lean-Rich Solvent Heat Exchanger

$\mathrm{Q}=$ Load (Btu/h)

Tha $=$ Inlet temperature of hot fluid $\left({ }^{\circ} \mathrm{F}\right)$

$12,184,945$

$\mathrm{Thb}=$ Outlet temperature of hot fluid $\left({ }^{\circ} \mathrm{F}\right)$

153

$-10$

Pressure of hot gases (psia)

Tca $=$ Inlet temperature of cold fluid $\left({ }^{\circ} \mathrm{F}\right)$

' -34

$\mathrm{Tcb}=$ Outlet temperature of cold fluid $\left({ }^{\circ} \mathrm{F}\right)$

Delta T1

Delta T2

Log mean temperature difference $\left({ }^{\circ} \mathrm{F}\right)$

Overall heat transfer coefficient $\left(\mathrm{Btu} / \mathrm{h} / \mathrm{t}^{2} /{ }^{\circ} \mathrm{F}\right)$

Heat transfer area $\left(\mathrm{ft}^{2}\right)$

Operating pressure (psia)

Pressure factor

Materials correction factor

1.175

Module factor

(includes all of the supporting equipment and connections and installation)

Purchased cost of heat exchanger in 1987

(mild steel construction, shell and tube floating head)

CE index for process equipment in 1987

CE index for process equipment in 1995

$\$ 54,000$

Installed cost of heat exchanger in 1995

\section{Solvent Refrigeration}

Refrigeration (tons)

Purchased cost in 1987

Temperature correction factor

Module factor

CE index for process equipment in 1987

CE index for process equipment in 1995

373.9

Installed cost of solvent refrigeration in 1995

Total Direct Cost 
TABLE 6.10 Sizing and Cost Estimation for Major Equipment Used for Fuel Cell System in Case 3

1. Fuel Gas Expansion Turbine

Turbine size (hp)

Purchased cost in 1979

Module factor

CE index for process equipment in 1979

CE index for process equipment in 1995

Installed cost of turbine in 1995

2. Heat Exchanger 1

$\mathrm{Q}=\operatorname{Load}($ Btu/h)

Tha $=$ Inlet temperature of hot fluid $\left({ }^{\circ} \mathrm{F}\right)$

$35,343,035$

667.23

100

Thb $=$ Outlet temperature of hot fluid $\left({ }^{\circ} \mathrm{F}\right)$

Pressure of hot gases (psia)

32.4

Tca $=$ Inlet temperature of cold fluid $\left({ }^{\circ} \mathrm{F}\right)$

600.00

$\mathrm{Tcb}=$ Outlet temperature of cold fluid $\left({ }^{\circ} \mathrm{F}\right)$

67.2315

Delta T2

Log mean temperature difference $\left({ }^{\circ} \mathrm{F}\right)$

Overall heat transfer coefficient $\left(\mathrm{Btu} / \mathrm{h} / \mathrm{ft} 2 /{ }^{\circ} \mathrm{F}\right)$

Heat transfer area $\left(\mathrm{ft}^{2}\right)$

Operating pressure (psia)

104,882

Pressure factor 145.00

Materials correction factor

1.16

Module factor

(includes all of the supporting equipment and connections and installation)

Purchased cost of heat exchanger in 1987

(mild steel construction, shell and tube floating head)

CE index for process equipment in 1987 


\section{Heat Exchanger 2}

$\mathrm{Q}=\operatorname{Load}($ Btu $/ \mathrm{h})$

Tha $=$ Inlet temperature of hot fluid $\left({ }^{\circ} \mathrm{F}\right)$

$239,973,908$

1300.00

Thb $=$ Outlet temperature of hot fluid $\left({ }^{\circ} \mathrm{F}\right)$

450

Pressure of hot gases (psia)

150

$\mathrm{Tca}=$ Inlet temperature of cold fluid $\left({ }^{\circ} \mathrm{F}\right)$

356.8

$\mathrm{Tcb}=$ Outlet temperature of cold fluid $\left({ }^{\circ} \mathrm{F}\right)$

775.00

Delta T1

525

Delta T2

Log mean temperature difference $\left({ }^{\circ} \mathrm{F}\right)$

250

Overall heat transfer coefficient $\left(\mathrm{Btu} / \mathrm{h} / \mathrm{ft}^{2} /{ }^{\circ} \mathrm{F}\right)$

Heat transfer area $\left(\mathrm{ft}^{2}\right)$

32,019

Operating pressure (psia)

146.96

Pressure factor

1.165

Materials correction factor

Module factor

(includes all of the supporting equipment and connections and installation)

Purchased cost of heat exchanger in 1987

(mild steel construction, shell and tube floating head)

CE index for process equipment in 1987

CE index for process equipment in 1995

Installed cost of heat exchanger in 1995

\section{Heat Exchanger 3}

$Q=\operatorname{Load}(B t u / h)$

Tha $=$ Inlet temperature of hot fluid $\left({ }^{\circ} \mathrm{F}\right)$

Thb $=$ Outlet temperature of hot fluid $\left({ }^{\circ} \mathrm{F}\right)$

Pressure of hot gases (psia)

Tca $=$ Inlet temperature of cold fluid $\left({ }^{\circ} \mathrm{F}\right.$ )

$\mathrm{Tcb}=$ Outlet temperature of cold fluid $\left({ }^{\circ} \mathrm{F}\right)$

Delta $T 1$

Delta T2

Log mean temperature difference $\left({ }^{\circ} \mathrm{F}\right)$

Overall heat transfer coefficient $\left(\mathrm{Btu} / \mathrm{h} / \mathrm{ft}^{2} /{ }^{\circ} \mathrm{F}\right)$

$224,212,870$

1411.87

980

150

356.8

356.77

1055.102336

624

820

30

Heat transfer area $\left(\mathrm{ft}^{2}\right)$

Operating pressure (psia)

9,109

146.96

1.165

Pressure factor

Module factor

(includes all of the supporting equipment and connections and installation)

Purchased cost of heat exchanger in 1987

(mild steel construction, shell and tube floating head)

CE index for process equipment in 1987

$\$ 100,000$

CE index for process equipment in 1995

Installed cost of heat exchanger in 1995 
TABLE 6.10 (Cont.)

5. Heat Exchanger 4

$Q=\operatorname{Load}(B t u / h)$

Tha $=$ Inlet temperature of hot fluid $\left({ }^{\circ} \mathrm{F}\right)$

$\mathrm{Thb}=$ Outlet temperature of hot fluid $\left({ }^{\circ} \mathrm{F}\right)$

Pressure of hot gases (psia)

$\mathrm{Tca}=$ Inlet temperature of cold fluid $\left({ }^{\circ} \mathrm{F}\right)$

$\mathrm{Tcb}=$ Outlet temperature of cold fluid $\left({ }^{\circ} \mathrm{F}\right)$

Delta T1

Delta T2

Log mean temperature difference $\left({ }^{\circ} \mathrm{F}\right)$

Overall heat transfer coefficient $\left(\mathrm{Btu} / \mathrm{h} / \mathrm{ft}^{2} /{ }^{\circ} \mathrm{F}\right)$

Heat transfer area $\left(\mathrm{ft}^{2}\right)$

Operating pressure (psia)

Pressure factor

Materials correction factor

Module factor

(includes all of the supporting equipment and connections and installation)

Purchased cost of heat exchanger in 1987

(mild steel construction, shell and tube floating head)

CE index for process equipment in 1987

CE index for process equipment in 1995

Installed cost of heat exchanger in 1995

$89,614,102$

667.23

400

15

356.8

356.79

310.4448363

43

136

30

22,038

146.96

1.165

1

3.2

$\$ 180,000$

320

373.9

$310,699,095$

450.00

150

150

121.4

356.77

93.23133627

29

55

30

189,274

146.96

1.165

1

3.2

$\$ 946,369$

320

373.9

$\$ 784,068$

Module factor

(includes all of the supporting equipment and connections and installation)

Purchased cost of heat exchanger in 1987

(mild steel construction, shell and tube floating head)

CE index for process equipment in 1987

CE index for process equipment in 1995

Installed cost of heat exchanger in 1995 
TABLE 6.10 (Cont.)

7. Refrigeration

Refrigeration (tons)

Purchased cost in 1987

700,000

Temperature correction factor

Module factor

CE index for process equipment in 1987

CE index for process equipment in 1995

373.9

Installed cost of solvent refrigeration in 1995

$\$ 1,194,143$

8. Cathode Exhaust Gas Expansion Turbine

Turbine size (hp)

Purchased cost in 1987

Module factor

104,234

$\$ 10,435,839$

1.00

320

CE index for process equipment in 1987

CE index for process equipment in 1995

373.9

Installed cost of turbine in 1995

9. Air Compressor for Fuel Cell

Inlet pressure (psia)

Outlet pressure (psia)

14.70

150.00

Compressor size (MW)

225

Purchased cost in 1987

Module factor

CE index for process equipment in 1987

CE index for process equipment in 1995

Installed cost air compressor in 1995

$\$ 24,446,768$

1.00

320

373.9

$\$ 28,564,520$

10. Steam Turbine

Turbine output (MW)

The cost of steam turbine is already included in the base case. 
11. Condenser

$\mathrm{Q}=\mathrm{Load}(\mathrm{Btu} / \mathrm{h})$

Tha $=$ Inlet temperature of hot fluid $\left({ }^{\circ} \mathrm{F}\right)$

Thb = Outlet temperature of hot fluid $\left({ }^{\circ} \mathrm{F}\right)$

Pressure of hot gases (psia)

$\mathrm{Tca}=$ Inlet temperature of cold fluid $\left({ }^{\circ} \mathrm{F}\right)$

$\mathrm{Tcb}=$ Outlet temperature of cold fluid $\left({ }^{\circ} \mathrm{F}\right)$

Delta T1

Delta T2

Log mean temperature difference $\left({ }^{\circ} \mathrm{F}\right)$

Overall heat transfer coefficient $\left(\mathrm{Btu} / \mathrm{h} / \mathrm{ft}^{2} /{ }^{\circ} \mathrm{F}\right)$

Heat transfer area $\left(\mathrm{ft}^{2}\right)$

Operating pressure (psia)

Pressure factor

Materials correction factor

Module factor

(includes all of the supporting equipment and connections and installation)

Purchased cost of heat exchanger in 1987

(mild steel construction, shell and tube floating head)

CE index for process equipment in 1987

CE index for process equipment in 1995

$420,802,598$

121.36

121

2

70.0

100.00

21.35924367

51

34

500

24,613

146.96

1.165

1

3.2

Installed cost of heat exchanger in 1995

$\$ 200,000$

320

373.9

$\$ 871,187$

12. Pump

Horsepower

Size exponent

110

Purchased cost of pump in 1987

(includes motor, coupling, base; cast iron, horizontal)

Module factor

CE index in 1987

CE index in 1995

Installed cost of solvent pump in 1995

13. Fuel Cell Stack

Fuel cell power output (kW)

Unit cost per kilowatt

Total cost 
TABLE 6.10 (Cont.)

14. Fuel Cell Invertor

Unit cost per kilowatt

$\$ 100$

Total cost

$\$ 7,795,216$

15. Fuel Cell Controls

Unit cost per kilowatt

$\$ 140$

Total cost

$\$ 10,913,302$

16. Fuel Cell and Components Assembly

Unit cost per kilowatt

$\$ 110$

Total cost

$\$ 8,574,737$

Total Direct Cost

$\$ 95,212,358$

Total Direct Cost for Three Trains 
TABLE 6.11 Sizing and Cost Estimation for Major Equipment Used for $\mathrm{CO}_{2}$ Removal in Glycol Process in Case 3

1. Gas-Gas Heat Exchanger

$Q=$ Load (Btu/h)

Tha $=$ Inlet temperature of hot fluid $\left({ }^{\circ} \mathrm{F}\right)$

$1,559,898$

$\mathrm{Thb}=$ Outlet temperature of hot fluid $\left({ }^{\circ} \mathrm{F}\right)$

70.00

Pressure of hot gases (psia)

55

150.00

Tca $=$ Inlet temperature of cold fluid $\left({ }^{\circ} \mathrm{F}\right)$

30.00

$\mathrm{Tcb}=$ Outlet temperature of cold fluid $\left({ }^{\circ} \mathrm{F}\right)$

56.14

Delta $T 1$

13.8564

Delta T2

25

19

Overall heat transfer coefficient (Btu/h/ $\left./ \mathrm{ft}^{2} /{ }^{\circ} \mathrm{F}\right)$

Heat transfer area $\left(\mathrm{ft}^{2}\right)$

16,521

Operating pressure (psia)

150.00

Pressure factor

1.16

Materials correction factor

Module factor

(includes all of the supporting equipment and connections and

installation)

Purchased cost of heat exchanger in 1987

(mild steel construction, shell and tube floating head)

CE index for process equipment in 1987

CE index for process equipment in 1993

Installed cost of heat exchanger in 1993

$\$ 160,000$

2. $\mathrm{CO}_{2}$ Absorption Column

Diameter of tower $(\mathrm{ft})$

HETP (ft)

Number of theoretical stages

Absorber tower height $(\mathrm{ft})$

( $4 \mathrm{ft}$ for inlet, outlet and gas, and liquid distributions)

Volume of packing $\left(\mathrm{ft}^{3}\right)$

Pressure factor

Cost per foot of column height

(mild steel construction)

Materials correction factor

Module factor

CE index for process equipment in 1987

CE index for process equipment in 1993

Installed cost of absorber in 1993

Cost of packing per cubic foot

(2-in. pall rings-metal)

Total cost of packing

$\$ 272,199$

$\$ 63.5$

4.16

320

360.4

$\$ 459,813$ 
TABLE 6.11 (Cont.)

3. Power Recovery Turbine 1

Turbine size (hp)

451

Purchased cost in 1979

$\$ 180,000$

Module factor

CE index for process equipment in 1987

320

CE index for process equipment in 1993

360.4

Installed cost of solvent pump in 1993

$\$ 210,319$

4. Slump Tank

Glycol flow rate $(\mathrm{lb} / \mathrm{h})$

Density of glycol (lb/gal)

$5,824,796$

Residence time (s)

8.6

Slump tank volume (gal)

180

Pressure factor

33,865

1.38

Materials correction factor

Module factor

2.08

Purchased cost of slump tank in 1987

$\$ 65,000$

(mild steel construction)

CE index for process equipment in 1987

320

CE index for process equipment in 1993

360.4

Installed cost of slump tank in 1993

$\$ 218,002$

\section{Recycle Compressor}

Inlet pressure (psia)

50

Outlet pressure (psia)

150.00

Compressor size (hp)

259

Purchased cost of centrifugal compressor in 1987

$\$ 95,000$

(includes electric motor drive and gear reducer)

Size factor

Materials correction factor

Module factor

CE index for process equipment in 1987

320

CE index for process equipment in 1993

360.4

Installed cost of compressor in 1993 
6. Flash Tank 1

Glycol flow rate $(\mathrm{lb} / \mathrm{h})$

$5,824,796$

Density of glycol (lb/gal)

Residence time (s)

Slump tank volume (gal)

33,865

Pressure factor

Materials correction factor

Module factor

2.08

Purchased cost of slump tank in 1987

$\$ 65,000$

(mild steel construction)

CE index for process equipment in 1987

320

CE index for process equipment in 1993

360.4

Installed cost of slump tank in 1993

$\$ 157,973$

7. Flash Tank 2

Glycol flow rate $(\mathrm{lb} / \mathrm{h})$

Density of glycol (lb/gal)

Residence time (s)

Slump tank volume (gal)

Pressure factor

Materials correction factor

Module factor

$5,824,796$

8.6

180

33,865

1

Purchased cost of slump tank in 1987

$\$ 65,000$

(mild steel construction)

CE index for process equipment in 1987

320

360.4

Installed cost of slump tank in 1993

$\$ 157,973$

8. Flash Tank 3

Glycol flow rate $(\mathrm{lb} / \mathrm{h})$

Density of glycol (lb/gal)

$5,824,796$

8.6

Residence time (s)

180

Slump tank volume (gal)

33,865

Pressure factor

Materials correction factor

1

Module factor

Purchased cost of slump tank in 1987

$\$ 65,000$

(mild steel construction)

CE index for process equipment in 1987

320

CE index for process equipment in 1993

360.4

Installed cost of slump tank in 1993 
9. Solvent Circulation Pump

Horsepower

1,282

Size exponent

0.79

Purchased cost of 300-hp pump in 1987

$\$ 30,000$

(includes motor, coupling, base; cast iron, horizontal)

Module factor

1.5

CE index for process equipment in 1987

320

CE index for process equipment in 1993

360.4

Installed cost of solvent pump in 1993

$\$ 165,631$

10. Compressor 1 for $\mathrm{CO}_{2}$

Inlet pressure (psia)

14.70

Outlet pressure (psia)

50.00

Compressor size (hp)

600.41

Purchased cost of centrifugal compressor in 1987

$\$ 85,000$

Size factor

Materials correction factor

1

Module factor

2.6

CE index for process equipment in 1987

320

CE index for process equipment in 1993

360.4

Installed cost of compressor in 1993

$\$ 258,225$

11. Compressor 2 for $\mathrm{CO}_{2}$

Inlet pressure (psia)

Outlet pressure (psia)

4.00

50.00

Compressor size (hp)

120.54

Purchased cost of centrifugal compressor in 1987

$\$ 50,000$

(includes electric motor drive and gear reducer)

Size factor

Materials correction factor

Module factor

CE index for process equipment in 1987

$C E$ index for process equipment in 1993

360.4

Installed cost of compressor in 1993

$\$ 151,897$

12. Solvent Refrigeration

Refrigeration (tons)

434.53

Purchased cost in 1987

$\$ 230,000$

Temperature correction factor

1.25

1.46

Module factor

320

CE index for process equipment in 1987

360.4

CE index for process equipment in 1993

$\$ 490,452$

Installed cost of solvent refrigeration in 1993 
TABLE 6.11 (Cont.)

13. $\mathrm{CO}_{2}$ Product Gas Compressors

Compressor 1 (hp)

$2,913.98$

Compressor $2(\mathrm{hp})$

$2,913.98$

Compressor $3(\mathrm{hp})$

$2,913.98$

Purchased cost of centrifugal compressor 1 in 1987

Purchased cost of centrifugal compressor 2 in 1987

Purchased cost of centrifugal compressor 3 in 1987

(includes electric motor drive and gear reducer)

Size factor

$\$ 750,000$

$\$ 750,000$

$\$ 750,000$

Module factor

CE index for process equipment in 1987

CE index for process equipment in 1993

360.4

Installed cost of compressor 1 in 1993

Installed cost of compressor 2 in 1993

$2,278,453$

$2,278,453$

Installed cost of compressor 3 in 1993

Total Direct Cost

$\$ 10,518,378$

Total Direct Cost for Three Trains 


\section{Case 4 - Fuel Cell Topping Cycle and Membrane $\mathrm{CO}_{2}$ Recovery}

Material and energy balances have been developed in this section for the application of an internal reforming molten carbonate fuel cell as the topping cycle for an IGCC plant. The $\mathrm{CO}_{2}$ from the fuel cell exhaust is recovered by membrane separation. The analysis is very similar to that presented in Section 6, except the glycol-based absorption system is replaced with a membrane system.

\subsection{Design Basis}

Figure 7.1 provides an overview of the of the IGCC system, including the gasifier, gas treatment, the fuel cell, and the steam cycle. This system is identical to that represented in Figure 6.1 and is reproduced here for convenience. The overall design of the fuel cell is determined by the gasifier capacity and synthesis gas composition. These are assumed to be the same as in the base case, which has no $\mathrm{CO}_{2}$ recovery. The fuel cell has very low tolerance for contaminants, including particulates and sulfur compounds. To achieve the required level of $\mathrm{H}_{2} \mathrm{~S}$ removal, a chilled methanol system has been employed rather than the glycol system used in the gas turbine cases. The chilled methanol system is designed to reduce the sulfur species $\left(\mathrm{H}_{2} \mathrm{~S}\right.$ and $\mathrm{COS}$ ) concentration to less than $1 \mathrm{ppmv}$. The reactions in the fuel cell anode shift the synthesis gas to a hydrogen-rich gas with a high concentration of $\mathrm{CO}_{2}$ and reduce the resultant hydrogen with carbonate ion. Oxidation of the carbonate at the anode releases $\mathrm{CO}_{2}$ and two electrons. The $\mathrm{CO}_{2}$-rich anode exhaust is treated in a membrane recovery system to separate most of the $\mathrm{CO}_{2}$. Thermal energy released by cooling this anode exhaust provides heat for the steam bottoming cycle. An expansion turbine is used on the cathode exhaust to extract energy.

Table 7.1 is a summary of principal material flows for the base case and for this design option. The $\mathrm{CO}_{2}$ reduction accomplished at the power plant is $89 \%$ and is accompanied by a $24 \%$ reduction in net electrical output from the base case, which uses a gas turbine and no $\mathrm{CO}_{2}$ recovery. A full accounting of the net $\mathrm{CO}_{2}$ reduction would include $\mathrm{CO}_{2}$ released in the generation of replacement power; mining, coal, and reagent preparation; and materials transport.

\subsection{Chilled Methanol Process for $\mathrm{H}_{2}$ S Recovery}

The design of the chilled methanol system is the same as that described for Case 3. It is required to provide adequate $\mathrm{H}_{2} \mathrm{~S}$ removal to meet fuel cell requirements. See Figure 6.2 and Tables 6.2 and 6.3 for details. 


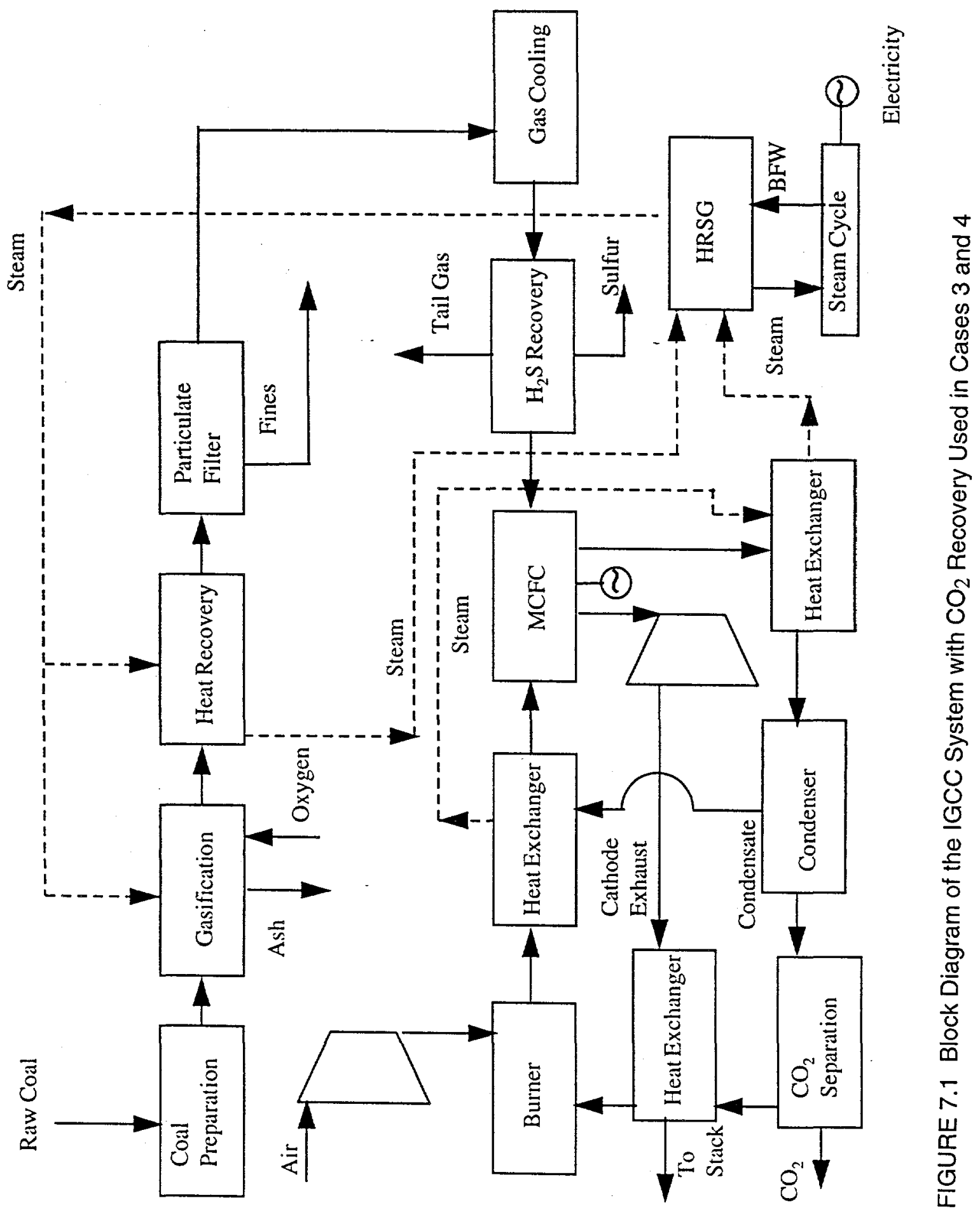


TABLE 7.1 Material Flows for Oxygen-Blown Base Case and Case 4

\begin{tabular}{|c|c|c|}
\hline Material Flow (tons/d) & Base Case & Case 4 \\
\hline Coal (prepared) & 3,845 & 3,845 \\
\hline Oxygen & 2,347 & 2,347 \\
\hline Solid waste & 492 & 492 \\
\hline Sulfur & 78 & 78 \\
\hline $\mathrm{CO}_{2}$ (power plant only) & 9210 & 993 \\
\hline $\mathrm{SO}_{2}$ (power plant only) & 1.08 & 6.92 \\
\hline Net power output (MW) & 413.5 & 313.77 \\
\hline
\end{tabular}

\subsection{Molten Carbonate Fuel Cell System}

The molten carbonate fuel cell system with the membrane for $\mathrm{CO}_{2}$ recovery is virtually identical to that described in section 6.3. A slight difference in stream composition following the membrane system reflects the performance difference between the two $\mathrm{CO}_{2}$-recovery systems. Table 7.2 is an alternate line list for Figure 6.3 detailing these differences.

\subsection{Membrane System for $\mathrm{CO}_{2}$ Recovery}

Figure 7.2 is an overall flow diagram of a membrane $\mathrm{CO}_{2}$-recovery system. It is similar to the membrane system described in Section 5. Table 7.3 is a line list corresponding to Figure 7.2. Stream descriptions and associated assumptions are provided in Table 7.4.

\subsection{Fuel Cell, Steam Cycle, and Plant Performance}

Use of the fuel cell topping cycle with methanol-based $\mathrm{H}_{2} \mathrm{~S}$ recovery and membrane $\mathrm{CO}_{2}$ recovery results in a net plant output of $314 \mathrm{MW}, 24 \%$ less than in the base case plant without $\mathrm{CO}_{2}$ recovery. Table 7.5 lists the topping cycle output, steam cycle output, and internal plant consumption for the base case (no $\mathrm{CO}_{2}$ recovery) and for the current case, Case 4 . The most significant losses are the consumption of power for $\mathrm{CO}_{2}$ compression and power required for permeate compression between membrane stages.

\subsection{Economics}

Details of the capital investment estimates for the $\mathrm{H}_{2} \mathrm{~S}$ recovery system, the fuel cell system, and the $\mathrm{CO}_{2}$ recovery system are presented in Tables 6.9, 7.6, and 7.7, respectively. A summary of capital costs, including indirect capital investment, operating, and maintenance costs, is provided in Section 9. 
TABLE 7.2 Stream Flows of Molten Carbonate Fuel Cell System in Case 4

\begin{tabular}{|c|c|c|c|c|c|c|}
\hline Stream Data & Stream 12 & Stream 13 & Stream 14 & Stream 15 & Stream 16 & Stream 17 \\
\hline Description of stream & $\begin{array}{l}\text { Gases to } \\
\text { membrane process }\end{array}$ & $\begin{array}{l}\text { Gases from } \\
\text { membrane process }\end{array}$ & $\begin{array}{l}\text { Gases from heat } \\
\text { exchanger } 1\end{array}$ & Air to compressor & $\begin{array}{l}\text { Air from } \\
\text { compressor }\end{array}$ & $\begin{array}{l}\text { Gases from } \\
\text { burner }\end{array}$ \\
\hline \multicolumn{7}{|l|}{ Gases $(\mathrm{lb} \cdot \mathrm{mol} / \mathrm{h})$} \\
\hline $\mathrm{CO}$ & $1,812.26$ & $1,539.74$ & $1,539.74$ & 0.00 & 0.00 & 0.00 \\
\hline $\mathrm{CO}_{2}$ & $8,724.66$ & $4,180.59$ & $4,180.59$ & 0.00 & 0.00 & $5,720.33$ \\
\hline $\mathrm{H}_{2}$ & $1,693.50$ & $1,692.69$ & $1,692.69$ & 0.00 & 0.00 & 0.00 \\
\hline $\mathrm{H}_{2} \mathrm{O}$ & 135.79 & 24.61 & 24.61 & 0.00 & 0.00 & $1,717.29$ \\
\hline $\mathrm{N}_{2}$ & 36.44 & 35.82 & 35.82 & $44,039.07$ & $44,039.07$ & $44,074.89$ \\
\hline $\mathrm{Ar}$ & 72.73 & 62.12 & 62.12 & 541.05 & 541.05 & 603.17 \\
\hline $\mathrm{CH}_{4}$ & 0.00 & 0.00 & 0.00 & 0.00 & 0.00 & 0.00 \\
\hline $\mathrm{NH}_{3}$ & 0.00 & 0.00 & 0.00 & 0.00 & 0.00 & 0.00 \\
\hline $\mathrm{H}_{2} \mathrm{~S}$ & 0.00 & 0.00 & 0.00 & 0.00 & 0.00 & 0.00 \\
\hline $\mathrm{HCN}$ & 0.00 & 0.00 & 0.00 & 0.00 & 0.00 & 0.00 \\
\hline $\mathrm{O}_{2}$ & 0.00 & 0.00 & 0.00 & $11,779.07$ & $11,779.07$ & $10,162.86$ \\
\hline $\cos$ & 0.00 & 0.00 & 0.00 & 0.00 & 0.00 & 0.00 \\
\hline $\mathrm{SO}_{2}$ & 0.00 & 0.00 & 0.00 & 0.00 & 0.00 & 0.00 \\
\hline Total gas flow & $12,475.38$ & $7,535.57$ & $7,535.57$ & $56,359.19$ & $56,359.19$ & $62,278.54$ \\
\hline \multicolumn{7}{|l|}{ Liquids (lb.mol/h) } \\
\hline $\mathrm{H}_{2} \mathrm{O}$ & 0.00 & 0.00 & 0.00 & 0.00 & 0.00 & 0.00 \\
\hline Temperature $\left({ }^{\circ} \mathrm{F}\right)$ & 150.00 & 154.88 & 600.00 & 81.00 & 713.05 & $1,355.19$ \\
\hline Pressure (psia) & 150.00 & 140.00 & 140.00 & 14.70 & 150.00 & 150.00 \\
\hline $\begin{array}{l}\text { Enthalpy of stream }(\mathrm{Btu} / \mathrm{h}) \\
\text { (reference, } 32^{\circ} \mathrm{F} \text { ) }\end{array}$ & $12,463,567$ & $7,542,911$ & $37,780,990$ & $18,972,575$ & $274,226,598$ & $676,482,506$ \\
\hline
\end{tabular}


TABLE 7.2 (Cont.)

\begin{tabular}{|c|c|c|c|c|c|c|}
\hline Stream Data & Stream 18 & Stream 19 & Stream 20 & Stream 21 & Stream 22 & Stream 23 \\
\hline Description of stream & $\begin{array}{l}\text { Gases from heat } \\
\text { exchanger } 3\end{array}$ & $\begin{array}{l}\text { Fuel cell cathode } \\
\text { exhaust }\end{array}$ & $\begin{array}{l}\text { Gases from } \\
\text { expansion turbine }\end{array}$ & $\begin{array}{l}\text { Gases from } \\
\text { splitter to heat } \\
\text { exchanger } 1\end{array}$ & $\begin{array}{l}\text { Gases from heat } \\
\text { exchanger } 1\end{array}$ & $\begin{array}{l}\text { Gases from } \\
\text { splitter to heat } \\
\text { exchanger } 4\end{array}$ \\
\hline \multicolumn{7}{|l|}{ Gases (lb.mol/h) } \\
\hline $\mathrm{CO}$ & 0.00 & 0.00 & 0.00 & 0.00 & 0.00 & 0.00 \\
\hline $\mathrm{CO}_{2}$ & $5,720.33$ & 461.70 & 461.70 & 75.84 & 75.84 & 385.85 \\
\hline $\mathrm{H}_{2}$ & 0.00 & 0.00 & 0.00 & 0.00 & 0.00 & 0.00 \\
\hline $\mathrm{H}_{2} \mathrm{O}$ & $1,717.29$ & $1,717.29$ & $1,717.29$ & 282.10 & 282.10 & $1,435.19$ \\
\hline $\mathrm{N}_{2}$ & $44,074.89$ & $44,074.89$ & $44,074.89$ & $7,240.25$ & $7,240.25$ & $36,834.64$ \\
\hline $\operatorname{Ar}$ & 603.17 & 603.17 & 603.17 & 99.08 & 99.08 & 504.08 \\
\hline $\mathrm{CH}_{4}$ & 0.00 & 0.00 & 0.00 & 0.00 & 0.00 & 0.00 \\
\hline $\mathrm{NH}_{3}$ & 0.00 & 0.00 & 0.00 & 0.00 & 0.00 & 0.00 \\
\hline $\mathrm{H}_{2} \mathrm{~S}$ & 0.00 & 0.00 & 0.00 & 0.00 & 0.00 & 0.00 \\
\hline $\mathrm{HCN}$ & 0.00 & 0.00 & 0.00 & 0.00 & 0.00 & 0.00 \\
\hline $\mathrm{O}_{2}$ & $10,162.86$ & $7,533.54$ & $7,533.54$ & $1,237.55$ & $1,237.55$ & $6,295.99$ \\
\hline $\cos$ & 0.00 & 0.00 & 0.00 & 0.00 & 0.00 & 0.00 \\
\hline $\mathrm{SO}_{2}$ & 0.00 & 0.00 & 0.00 & 0.00 & 0.00 & 0.00 \\
\hline Total gas flow & $62,278.54$ & $54,390.59$ & $54,390.59$ & $8,934.82$ & $8,934.82$ & $45,455.75$ \\
\hline \multicolumn{7}{|l|}{ Liquids (lb-mol/h) } \\
\hline $\mathrm{H}_{2} \mathrm{O}$ & 0.00 & 0.00 & 0.00 & 0.00 & 0.00 & 0.00 \\
\hline Temperature $\left({ }^{\circ} \mathrm{F}\right)$ & 980.33 & $1,300.00$ & 667.24 & 667.24 & 200.00 & 667.24 \\
\hline Pressure (psia) & 150.00 & 150.00 & 14.70 & 14.70 & 14.70 & 14.70 \\
\hline $\begin{array}{l}\text { Enthalpy of stream }(B t u / h) \\
\text { (reference, } 32^{\circ} \mathrm{F} \text { ) }\end{array}$ & $482,489,027$ & $546,065,398$ & $281,008,417$ & $46,161,714$ & $15,923,634$ & $234,846,704$ \\
\hline
\end{tabular}


TABLE 7.2 (Cont.)

\begin{tabular}{|c|c|c|c|c|c|c|}
\hline Stream Data & Stream 24 & Stream 25 & Stream 26 & Stream 27 & Stream 28 & Stream 29 \\
\hline Description of stream & $\begin{array}{l}\text { Gases from heat } \\
\text { exchanger } 4\end{array}$ & $\begin{array}{l}\text { Water from } \\
\text { condenser }\end{array}$ & $\begin{array}{l}\text { Water from } \\
\text { pump }\end{array}$ & $\begin{array}{l}\text { Steam from heat } \\
\text { exchanger } 5\end{array}$ & $\begin{array}{l}\text { Steam from heat } \\
\text { exchanger } 4\end{array}$ & $\begin{array}{l}\text { Steam from heat } \\
\text { exchanger } 3\end{array}$ \\
\hline \multicolumn{7}{|l|}{ Gases $(\mathrm{lb} \cdot \mathrm{mol} / \mathrm{h})$} \\
\hline $\mathrm{CO}$ & 0.00 & 0.00 & 0.00 & 0.00 & 0.00 & 0.00 \\
\hline $\mathrm{CO}_{2}$ & 385.85 & 0.00 & 0.00 & 0.00 & 0.00 & 0.00 \\
\hline $\mathrm{H}_{2}$ & 0.00 & 0.00 & 0.00 & 0.00 & 0.00 & 0.00 \\
\hline $\mathrm{H}_{2} \mathrm{O}$ & $1,435.19$ & 0.00 & 0.00 & $10,270.88$ & $15,985.92$ & $28,434.17$ \\
\hline $\mathrm{N}_{2}$ & $36,834.64$ & 0.00 & 0.00 & 0.00 & 0.00 & 0.00 \\
\hline $\mathrm{Ar}$ & 504.08 & 0.00 & 0.00 & 0.00 & 0.00 & 0.00 \\
\hline $\mathrm{CH}_{4}$ & 0.00 & 0.00 & 0.00 & 0.00 & 0.00 & 0.00 \\
\hline $\mathrm{NH}_{3}$ & 0.00 & 0.00 & 0.00 & 0.00 & 0.00 & 0.00 \\
\hline $\mathrm{H}_{2} \mathrm{~S}$ & 0.00 & 0.00 & 0.00 & 0.00 & 0.00 & 0.00 \\
\hline $\mathrm{HCN}$ & 0.00 & 0.00 & 0.00 & 0.00 & 0.00 & 0.00 \\
\hline $\mathrm{O}_{2}$ & $6,295.99$ & 0.00 & 0.00 & 0.00 & 0.00 & 0.00 \\
\hline $\cos$ & 0.00 & 0.00 & 0.00 & 0.00 & 0.00 & 0.00 \\
\hline $\mathrm{SO}_{2}$ & 0.00 & 0.00 & 0.00 & 0.00 & 0.00 & 0.00 \\
\hline Total gas flow & $45,455.75$ & 0.00 & 0.00 & $10,270.88$ & $15,985.92$ & $28,434.17$ \\
\hline \multicolumn{7}{|l|}{ Liquids (lb-mol/h) } \\
\hline $\mathrm{H}_{2} \mathrm{O}$ & 0.00 & $22,923.59$ & $34,923.59$ & $24,652.71$ & $18,937.68$ & $6,489.42$ \\
\hline Temperature ( ${ }^{\circ} \mathrm{F}$ ) & 400.00 & 121.36 & 121.36 & 356.77 & 356.79 & 356.77 \\
\hline Pressure (psia) & 14.70 & 1.76 & 146.96 & 146.96 & 146.96 & 146.96 \\
\hline $\begin{array}{l}\text { Enthalpy of stream }(B t u / h) \\
\text { (reference, } 32^{\circ} \mathrm{F} \text { ) }\end{array}$ & $145,783,678$ & $36,915,818$ & $56,173,167$ & $366,812,595$ & $455,875,619$ & $649,869,098$ \\
\hline
\end{tabular}


TABLE 7.2 (Cont.)

\begin{tabular}{|c|c|c|c|c|c|c|c|}
\hline Stream Data & Stream 30 & Stream 31 & Stream 32 & Stream 33 & Stream 34A & Stream 34B & Stream 34C \\
\hline Description of stream & $\begin{array}{l}\text { Steam from heat } \\
\text { exchanger } 2\end{array}$ & $\begin{array}{l}\text { Steam for heating } \\
\text { feed to fuel cell }\end{array}$ & $\begin{array}{l}\text { Steam to steam } \\
\text { turbine }\end{array}$ & $\begin{array}{l}\text { Steam turbine } \\
\text { exhaust }\end{array}$ & $\begin{array}{l}\text { Makeup water } \\
\text { to pump }\end{array}$ & $\begin{array}{l}\text { Makeup water } \\
\text { to pump }\end{array}$ & $\begin{array}{l}\text { Makeup water } \\
\text { to pump }\end{array}$ \\
\hline \multicolumn{8}{|l|}{ Gases $(\mathrm{lb} \cdot \mathrm{mol} / \mathrm{h})$} \\
\hline $\mathrm{CO}$ & 0.00 & 0.00 & 0.00 & 0.00 & 0.00 & 0.00 & 0.00 \\
\hline $\mathrm{CO}_{2}$ & 0.00 & 0.00 & 0.00 & 0.00 & 0.00 & 0.00 & 0.00 \\
\hline $\mathrm{H}_{2}$ & 0.00 & 0.00 & 0.00 & 0.00 & 0.00 & 0.00 & 0.00 \\
\hline $\mathrm{H}_{2} \mathrm{O}$ & $34,923.59$ & $12,000.00$ & $22,923.59$ & $21,596.52$ & 0.00 & 0.00 & 0.00 \\
\hline $\mathrm{N}_{2}$ & 0.00 & 0.00 & 0.00 & 0.00 & 0.00 & 0.00 & 0.00 \\
\hline $\operatorname{Ar}$ & 0.00 & 0.00 & 0.00 & 0.00 & 0.00 & 0.00 & 0.00 \\
\hline $\mathrm{CH}_{4}$ & 0.00 & 0.00 & 0.00 & 0.00 & 0.00 & 0.00 & 0.00 \\
\hline $\mathrm{NH}_{3}$ & 0.00 & 0.00 & 0.00 & 0.00 & 0.00 & 0.00 & 0.00 \\
\hline $\mathrm{H}_{2} \mathrm{~S}$ & 0.00 & 0.00 & 0.00 & 0.00 & 0.00 & 0.00 & 0.00 \\
\hline $\mathrm{HCN}$ & 0.00 & 0.00 & 0.00 & 0.00 & 0.00 & 0.00 & 0.00 \\
\hline $\mathrm{O}_{2}$ & 0.00 & 0.00 & 0.00 & 0.00 & 0.00 & 0.00 & 0.00 \\
\hline $\cos$ & 0.00 & 0.00 & 0.00 & 0.00 & 0.00 & 0.00 & 0.00 \\
\hline $\mathrm{SO}_{2}$ & 0.00 & 0.00 & 0.00 & 0.00 & 0.00 & 0.00 & 0.00 \\
\hline Total gas flow & $34,923.59$ & $12,000.00$ & $22,923.59$ & $21,596.52$ & 0.00 & 0.00 & 0.00 \\
\hline \multicolumn{8}{|l|}{ Liquids $(\mathrm{lb} \cdot \mathrm{mol} / \mathrm{h})$} \\
\hline $\mathrm{H}_{2} \mathrm{O}$ & 0.00 & 0.00 & 0.00 & $1,327.07$ & $13,443.34$ & $12,000.00$ & $1,443.34$ \\
\hline Temperature $\left({ }^{\circ} \mathrm{F}\right)$ & 775.00 & 775.00 & 775.00 & 121.36 & 150.00 & 150.00 & 150.00 \\
\hline Pressure (psia) & 146.96 & 146.96 & 146.96 & 1.76 & 146.96 & 146.96 & 146.96 \\
\hline $\begin{array}{l}\text { Enthalpy of stream }(\mathrm{Btu} / \mathrm{h}) \\
\left.\text { (reference, } 32^{\circ} \mathrm{F}\right)\end{array}$ & $889,843,009$ & $305,756,514$ & $584,086,495$ & $435,269,723$ & $28,553,650$ & $25,488,000$ & $3,065,650$ \\
\hline
\end{tabular}


12

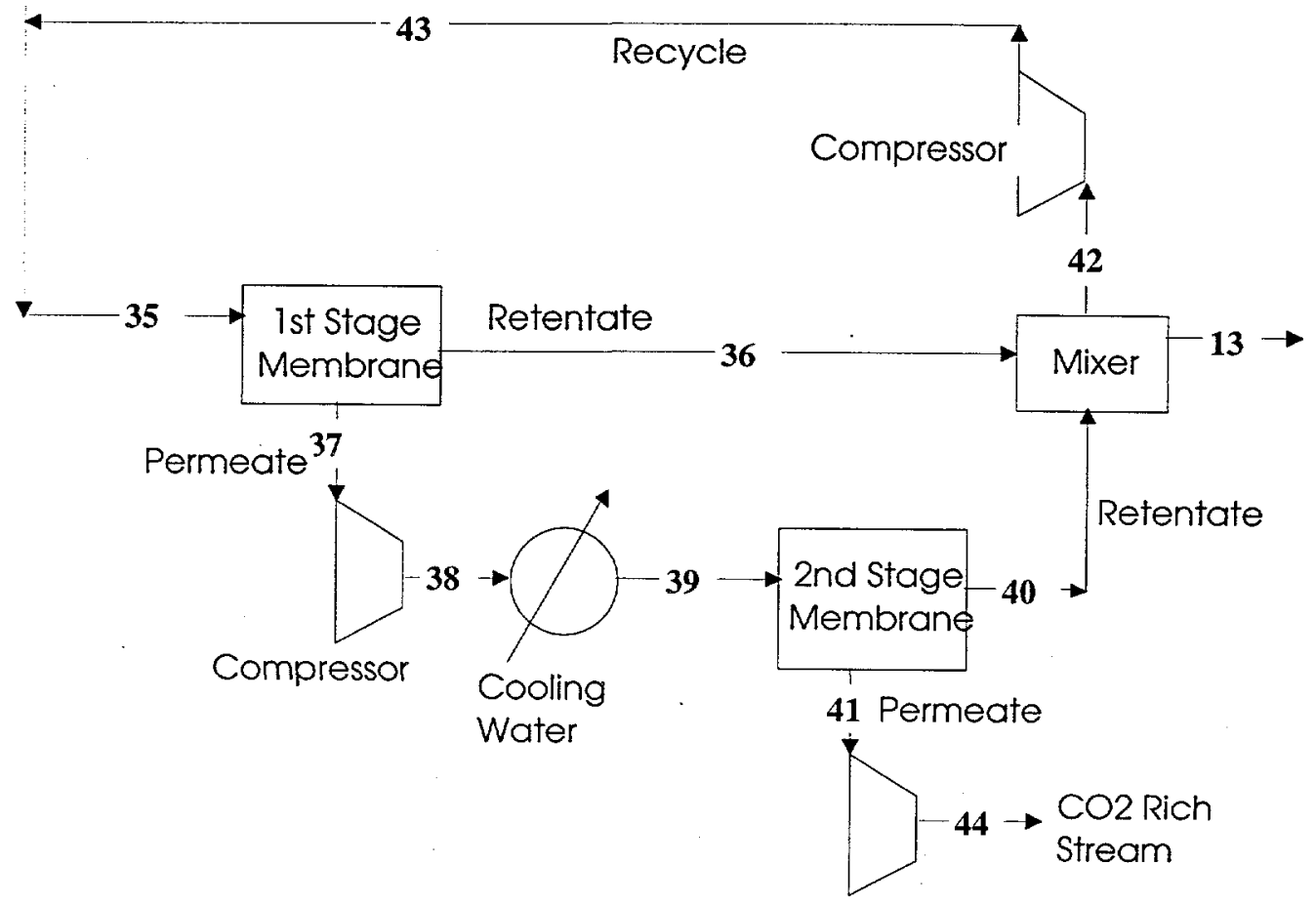

FIGURE 7.2 Flow Diagram of Membrane Process for $\mathrm{CO}_{2}$ Removal in Case 4 
TABLE 7.3 Stream Flows of Membrane Process for $\mathrm{CO}_{2}$ Removal in Case 4

\begin{tabular}{|c|c|c|c|c|c|c|}
\hline Stream Data & Stream 12 & Stream 13 & Stream 35 & Stream 36 & Stream 37 & Stream 38 \\
\hline Description of stream & $\begin{array}{l}\text { Feed gas from } \\
\text { fuel cell system }\end{array}$ & $\begin{array}{l}\mathrm{H}_{2} \text {-rich gas to } \\
\text { fuel cell }\end{array}$ & $\begin{array}{l}\text { To 1st-stage } \\
\text { membrane }\end{array}$ & 1 st-stage retentate & 1st-stage permeate & $\begin{array}{l}\text { Gases from } \\
\text { compressor }\end{array}$ \\
\hline \multicolumn{7}{|l|}{ Gases (lb·mol/h) } \\
\hline $\mathrm{CO}$ & $1,812.26$ & $1,539.74$ & $2,042.34$ & $1,296.30$ & 746.04 & 746.04 \\
\hline $\mathrm{CO}_{2}$ & $8,724.66$ & $4,180.59$ & $9,349.34$ & $2,831.36$ & $6,517.98$ & $6,517.98$ \\
\hline $\mathrm{H}_{2}$ & $1,693.50$ & $1,692.69$ & $1,946.43$ & $1,906.70$ & 39.72 & 39.72 \\
\hline $\mathrm{H}_{2} \mathrm{O}$ & 135.79 & 24.61 & 139.47 & 14.94 & 124.53 & 124.53 \\
\hline $\mathrm{N}_{2}$ & 36.44 & 35.82 & 41.79 & 36.72 & 5.07 & 5.07 \\
\hline $\mathrm{Ar}$ & 72.73 & 62.12 & 82.01 & 52.51 & 29.50 & 29.50 \\
\hline $\mathrm{CH}_{4}$ & 0.00 & 0.00 & 0.00 & 0.00 & 0.00 & 0.00 \\
\hline $\mathrm{NH}_{3}$ & 0.00 & 0.00 & 0.00 & 0.00 & 0.00 & 0.00 \\
\hline $\mathrm{H}_{2} \mathrm{~S}$ & 0.00 & 0.00 & 0.00 & 0.00 & 0.00 & 0.00 \\
\hline $\mathrm{HCN}$ & 0.00 & 0.00 & 0.00 & 0.00 & 0.00 & 0.00 \\
\hline $\mathrm{O}_{2}$ & 0.00 & 0.00 & 0.00 & 0.00 & 0.00 & 0.00 \\
\hline $\cos$ & 0.00 & 0.00 & 0.00 & 0.00 & 0.00 & 0.00 \\
\hline $\mathrm{SO}_{2}$ & 0.00 & 0.00 & 0.00 & 0.00 & 0.00 & 0.00 \\
\hline Total gas flow & $12,475.38$ & $7,535.57$ & $13,601.38$ & $6,138.53$ & $7,462.84$ & $7,462.84$ \\
\hline Liquids (lb·mol/h) & 0.00 & 0.00 & 0.00 & 0.00 & 0.00 & 0.00 \\
\hline Temperature $\left({ }^{\circ} \mathrm{F}\right)$ & 150.00 & 154.88 & 128.70 & 128.70 & 128.70 & 472.66 \\
\hline Pressure (psia) & 150.00 & 140.00 & 150.00 & 140.00 & 25.00 & 150.00 \\
\hline $\begin{array}{l}\text { Enthalpy of stream }(\mathrm{Btu} / \mathrm{h}) \\
\left.\text { (reference, } 32^{\circ} \mathrm{F}\right)\end{array}$ & $12,462,913$ & $7,542,574$ & $11,053,736$ & $4,694,179$ & $6,359,557$ & $31,178,763$ \\
\hline
\end{tabular}


TABLE 7.3 (Cont.)

\begin{tabular}{|c|c|c|c|c|c|}
\hline Stream Data & Stream 39 & Stream 40 & Stream 41 & Stream 42 & Stream 43 \\
\hline Description of stream & $\begin{array}{l}\text { Gases to 2nd- } \\
\text { stage membrane }\end{array}$ & 2nd-stage retentate & $\mathrm{CO}_{2}$-rich product gas & $\begin{array}{l}\text { Recycle gases to } \\
\text { compressor }\end{array}$ & $\begin{array}{l}\text { Recycle gases from } \\
\text { compressor }\end{array}$ \\
\hline \multicolumn{6}{|l|}{ Gases $(\mathrm{lb} \cdot \mathrm{mol} / \mathrm{h})$} \\
\hline $\mathrm{CO}$ & 746.04 & 473.52 & 272.52 & 230.08 & 230.08 \\
\hline $\mathrm{CO}_{2}$ & $6,517.98$ & $1,973.91$ & $4,544.07$ & 624.69 & 624.69 \\
\hline $\mathrm{H}_{2}$ & 39.72 & 38.91 & 0.81 & 252.93 & 252.93 \\
\hline $\mathrm{H}_{2} \mathrm{O}$ & 124.53 & 13.34 & 111.18 & 3.68 & 3.68 \\
\hline $\mathrm{N}_{2}$ & 5.07 & 4.46 & 0.62 & 5.35 & 5.35 \\
\hline Ar & 29.50 & 18.89 & 10.61 & 9.28 & 9.28 \\
\hline $\mathrm{CH}_{4}$ & 0.00 & 0.00 & 0.00 & 0.00 & 0.00 \\
\hline $\mathrm{NH}_{3}$ & 0.00 & 0.00 & 0.00 & 0.00 & 0.00 \\
\hline $\mathrm{H}_{2} \mathrm{~S}$ & 0.00 & 0.00 & 0.00 & 0.00 & 0.00 \\
\hline $\mathrm{HCN}$ & 0.00 & 0.00 & 0.00 & 0.00 & 0.00 \\
\hline $\mathrm{O}_{2}$ & 0.00 & 0.00 & 0.00 & 0.00 & 0.00 \\
\hline $\cos$ & 0.00 & 0.00 & 0.00 & 0.00 & 0.00 \\
\hline $\mathrm{SO}_{2}$ & 0.00 & 0.00 & 0.00 & 0.00 & 0.00 \\
\hline Total gas flow & $7,462.84$ & $2,523.03$ & $4,939.81$ & $1,126.01$ & $1,126.01$ \\
\hline Liquids (lb·mol/h) & 0.00 & 0.00 & 0.00 & 0.00 & 0.00 \\
\hline Temperature $\left({ }^{\circ} \mathrm{F}\right)$ & 212.00 & 212.00 & 212.00 & 154.88 & 167.34 \\
\hline Pressure (psia) & 150.00 & 140.00 & 25.00 & 140.00 & 150.00 \\
\hline $\begin{array}{l}\text { Enthalpy of stream }(B t u / h) \\
\text { (reference, } 32^{\circ} \mathrm{F} \text { ) }\end{array}$ & $12,062,267$ & $3,975,443$ & $8,086,823$ & $1,127,051$ & $1,243,809$ \\
\hline
\end{tabular}


TABLE 7.4 Descriptions of Streams of Membrane Process for $\mathrm{CO}_{2}$ Removal in Case 4

Stream and

Characteristics

Data

Comments on Stream Calculations

Stream 12: $\mathrm{CO}_{2}$-rich gas from

fuel cell section

Temperature $\left({ }^{\circ} \mathrm{F}\right)$

Pressure (psia)

Flow rate $(\mathrm{lb} \cdot \mathrm{mol} / \mathrm{h})$

$\mathrm{CO}_{2}$ (mole fraction)

150

150

$12,475.38$

0.6994

Stream 35: Feed gas to 1st-stage membrane system

Temperature $\left({ }^{\circ} \mathrm{F}\right)$

Pressure (psia)

Flow rate $(\mathrm{lb} \cdot \mathrm{mol} / \mathrm{h})$

$\mathrm{CO}_{2}$ (mole fraction)

Stream 36: Retentate from 1st-stage membrane system

Temperature $\left({ }^{\circ} \mathrm{F}\right)$

Pressure (psia)

Flow rate $(\mathrm{lb} \cdot \mathrm{mol} / \mathrm{h})$

$\mathrm{CO}_{2}$ (mole fraction)
128.70

150

$13,601.38$

0.6874

128.70

140

$6,138.53$

0.4612
The synthesis gas is cleaned in two stages. First, sulfur compounds are removed by chilled methanol. This is the sulfur-free system.

The sulfur-free gas is mixed with the recycle from the 2nd-stage retentate and fed to the 1st-stage membranes.

The composition of this stream depends on the permeability and selectivity of the membranes. The membrane system is a facilitated membrane that has a higher selectivity and permeability for $\mathrm{CO}_{2}$ than for $\mathrm{H}_{2}$. The ratio of permeate to retentate $\mathrm{CO}_{2}$ selectivity is 2.3 times for a pressure drop of 125 psia.

Stream 37: Permeate from 1st-stage membrane system

Temperature $\left({ }^{\circ} \mathrm{F}\right)$

Pressure (psia)

Flow rate $(\mathrm{lb} \cdot \mathrm{mol} / \mathrm{h})$

$\mathrm{CO}_{2}$ (mole fraction)

128.70 The composition of this stream is

25 calculated by mass balance around the

$7,462.84$ membrane.

Stream 38: Gases from compressor

Temperature ( $\left.{ }^{\circ} \mathrm{F}\right)$

Pressure (psia)

The permeate from the 1st-stage membrane

Flow rate $(\mathrm{lb} \cdot \mathrm{rnol} / \mathrm{h})$

$\mathrm{CO}_{2}$ (mole fraction) is at a pressure of $25 \mathrm{psia}$. These gases are again compressed to a pressure of 150 psia for the 2nd-stage membrane. 
TABLE 7.4 (Cont.)

\begin{tabular}{|c|c|c|}
\hline $\begin{array}{l}\text { Stream and } \\
\text { Characteristics }\end{array}$ & Data & Comments on Stream Calculations \\
\hline \multicolumn{3}{|c|}{ Stream 39: Gases from heat exchanger } \\
\hline Temperature $\left({ }^{\circ} \mathrm{F}\right)$ & 212 & The temperature of the gases rises because of \\
\hline Pressure (psia) & 150 & the compression. Therefore, this stream is \\
\hline Flow rate $(\mathrm{lb} \cdot \mathrm{mol} / \mathrm{h})$ & $7,462.84$ & cooled to a temperature of $212^{\circ} \mathrm{F}$, suitable \\
\hline $\mathrm{CO}_{2}$ (mole fraction) & 0.8734 & for the membrane system. \\
\hline \multicolumn{3}{|c|}{$\begin{array}{l}\text { Stream 40: Retentate of } 2 \text { nd-stage } \\
\text { membrane system }\end{array}$} \\
\hline Temperature $\left({ }^{\circ} \mathrm{F}\right)$ & 212 & The composition of this stream is \\
\hline Pressure (psia) & 140 & calculated on the basis of the selectivity and \\
\hline Flow rate $(\mathrm{lb} \cdot \mathrm{mol} / \mathrm{h})$ & $2,523.03$ & permeability of gases, as is done for stream \\
\hline $\mathrm{CO}_{2}$ (mole fraction) & 0.7824 & $\begin{array}{l}\text { 36. The ratio of permeate to retentate } \mathrm{CO}_{2} \\
\text { selectivity is } 2.3 \text { for a pressure drop of } \\
125 \text { psia. }\end{array}$ \\
\hline \multicolumn{3}{|c|}{$\begin{array}{l}\text { Stream 41: Permeate of } 2 \text { nd-stage } \\
\text { membrane system }\end{array}$} \\
\hline Temperature $\left({ }^{\circ} \mathrm{F}\right)$ & 212 & The composition of this stream is \\
\hline Pressure (psia) & 25 & calculated on the basis of the mass balance \\
\hline Flow rate $(\mathrm{lb} \cdot \mathrm{mol} / \mathrm{h})$ & $4,939.81$ & around the membrane. This is the $\mathrm{CO}_{2}$-rich \\
\hline $\mathrm{CO}_{2}$ (mole fraction) & 0.9199 & stream for disposal. \\
\hline \multicolumn{3}{|c|}{ Stream 13: Fuel gas to gas turbines } \\
\hline Temperature $\left({ }^{\circ} \mathrm{F}\right)$ & 154.88 & $\mathrm{H}_{2}$-rich retentate from 1st stage (stream 36 ) \\
\hline Pressure (psia) & 140 & and that from 2nd stage (stream 40) are mixed. \\
\hline Flow rate $(\mathrm{lb} \cdot \mathrm{mol} / \mathrm{h})$ & $7,535.57$ & Part of mixture is taken as fuel gas for gas \\
\hline $\mathrm{CO}_{2}$ (mole fraction) & 0.5548 & turbines. \\
\hline \multicolumn{3}{|c|}{$\begin{array}{l}\text { Stream 42: Recycle to } 1 \text { st-stage } \\
\text { membrane system }\end{array}$} \\
\hline Temperature $\left({ }^{\circ} \mathrm{F}\right)$ & 154.88 & Part of the retentate from stream 36 and part from \\
\hline Pressure (psia) & 140 & stream 40 are recycled back to the 1 st-stage \\
\hline Flow rate $(\mathrm{lb} \cdot \mathrm{mol} / \mathrm{h})$ & $1,126.01$ & membrane systems to increase the $\mathrm{CO}_{2}$ removal \\
\hline $\mathrm{CO}_{2}$ (mole fraction) & 0.5548 & efficiency. \\
\hline \multicolumn{3}{|c|}{$\begin{array}{l}\text { Stream 43: Recycle to } 1 \text { st-stage } \\
\text { membrane after compression }\end{array}$} \\
\hline Temperature $\left({ }^{\circ} \mathrm{F}\right)$ & 167.34 & The recycle from the retentate is at a \\
\hline Pressure (psia) & 150 & pressure of 150 psia and is compressed to \\
\hline Flow rate $(\mathrm{lb} \cdot \mathrm{mol} / \mathrm{h})$ & $1,126.01$ & the inlet pressure of the 1st membrane. \\
\hline $\mathrm{CO}_{2}$ (mole fraction) & 0.5548 & \\
\hline
\end{tabular}


TABLE 7.5 Power Output, Plant Power Use, and Net Power Output for Base Case and Case 4 Fuel Cell/ Membrane Process

\begin{tabular}{lcc}
\hline & \multicolumn{2}{c}{ Power (MW) } \\
\cline { 2 - 3 } Power Variable & Base Case & Fuel Cell Case \\
\hline & & \\
Power output & 298.8 & 247.4 \\
Gas turbine or fuel cell & 159.4 & 165.8 \\
Steam turbine & & \\
& & \\
Internal power consumption & 0 & 28.7 \\
CO $_{2}$ recovery & 0 & 0 \\
CO ${ }_{2}$ compression & 0 & -21.8 \\
Solvent circulation & 0 & -48.9 \\
Solvent refrigeration & -44.7 & 313.8 \\
Others & & \\
Gasification system & & \\
Net power output & 413.5 & 99.7 \\
Energy penalty & 0 & \\
\hline
\end{tabular}

a Includes $\mathrm{H}_{2} \mathrm{~S}$ recovery system energy use. 
TABLE 7.6 Sizing and Cost Estimation for Major Equipment Used for Fuel Cell System in Case 4

1. Fuel Gas Expansion Turbine

Turbine size (hp)

Purchased cost in 1979

Module factor

CE index for process equipment in 1979

CE index for process equipment in 1995

Installed cost of turbine in 1995

2,296
$\$ 1,607,439$
1.00
$\$ 256$
373.9

$\$ 2,347,740$

$30,238,080$

667.24

200

15

154.9

600.00

67.2395

Delta T1

Delta T2

Log mean temperature difference $\left({ }^{\circ} \mathrm{F}\right)$

Overall heat transfer coefficient $\left(\mathrm{Btu} / \mathrm{h} / \mathrm{ft}^{2} /{ }^{\circ} \mathrm{F}\right)$

Heat transfer area $\left(\mathrm{ft}^{2}\right)$

Operating pressure (psia)

Pressure factor

Materials correction factor

Module factor

(includes all of the supporting equipment and connections and installation)

Purchased cost of heat exchanger in 1987

(mild steel construction, shell and tube floating head)

CE index for process equipment in 1987

CE index for process equipment in 1995

Installed cost of heat exchanger in 1995

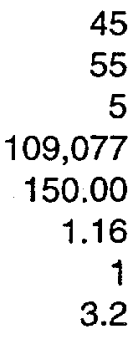

$\$ 545,385$

320

373.9 
3. Heat Exchanger 2

$\mathrm{Q}=\operatorname{Load}(\mathrm{Btu} / \mathrm{h})$

Tha $=$ inlet temperature of hot fluid $\left({ }^{\circ} \mathrm{F}\right)$

$\mathrm{Thb}=$ Outlet temperature of hot fluid $\left({ }^{\circ} \mathrm{F}\right)$

Pressure of hot gases (psia)

$\mathrm{Tca}=$ Inlet temperature of cold fluid $\left({ }^{\circ} \mathrm{F}\right)$

$\mathrm{Tcb}=$ Outlet temperature of cold fluid $\left({ }^{\circ} \mathrm{F}\right)$

Delta T1

Delta T2

Log mean temperature difference $\left({ }^{\circ} \mathrm{F}\right)$

$239,973,908$

1300.00

450

150

356.8

775.00

525

93

250

Overall heat transfer coefficient $\left(\mathrm{Btu} / \mathrm{h} / \mathrm{ft}^{2} /{ }^{\circ} \mathrm{F}\right)$

30

Heat transfer area $\left(\mathrm{ft}^{2}\right)$

32,019

Operating pressure (psia)

146.96

Pressure factor

1.165

Materials correction factor

Module factor

(includes all of the supporting equipment and connections and installation)

Purchased cost of heat exchanger in 1987

(mild steel construction, shell and tube floating head)

CE index for process equipment in 1987

$C E$ index for process equipment in 1995

Installed cost of heat exchanger in 1995

$\$ 250,000$

320

373.9

$\$ 1,088,984$

4. Heat Exchanger 3

$Q=\operatorname{Load}(\mathrm{Btu} / \mathrm{h})$

Tha $=$ Inlet temperature of hot fluid $\left({ }^{\circ} \mathrm{F}\right)$

$\mathrm{Thb}=$ Outlet temperature of hot fluid $\left({ }^{\circ} \mathrm{F}\right)$

Pressure of hot gases (psia)

Tca $=$ Inlet temperature of cold fluid $\left({ }^{\circ} \mathrm{F}\right)$

$\mathrm{Tcb}=$ Outlet temperature of cold fluid $\left({ }^{\circ} \mathrm{F}\right)$

Delta $T 1$

Delta T2

Log mean temperature difference $\left({ }^{\circ} \mathrm{F}\right)$

$193,993,479$

1355.19

980

150

356.8

356.77

998.4229363

624

796

Overall heat transfer coefficient (Btu/h/ft $\left.2 /{ }^{\circ} \mathrm{F}\right)$

30

8,120

Operating pressure (psia)

146.96

Pressure factor

1.165

Materials correction factor

Module factor

(includes all of the supporting equipment and connections and installation)

Purchased cost of heat exchanger in 1987

(mild steel construction, shell and tube floating head)

CE index for process equipment in 1987

CE index for process equipment in 1995

Installed cost of heat exchanger in 1995 


\section{Heat Exchanger 4}

$\mathrm{Q}=\operatorname{Load}(B t u / h)$

Tha $=$ Inlet temperature of hot fluid $\left({ }^{\circ} \mathrm{F}\right)$

$89,063,026$

667.24

400

$\mathrm{Thb}=$ Outlet temperature of hot fluid $\left({ }^{\circ} \mathrm{F}\right)$

Pressure of hot gases (psia)

356.8

Tca $=$ Inlet temperature of cold fluid $\left({ }^{\circ} \mathrm{F}\right)$

356.79

$\mathrm{Tcb}=$ Outlet temperature of cold fluid $\left({ }^{\circ} \mathrm{F}\right)$

528363

Delta T1

Delta T2

Log mean temperature difference $\left({ }^{\circ} \mathrm{F}\right)$

Overall heat transfer coefficient $\left(\mathrm{Btu} / \mathrm{h} / \mathrm{ft}^{2} /{ }^{\circ} \mathrm{F}\right)$

Heat transfer area $\left(\mathrm{ft}^{2}\right)$

Operating pressure (psia)

Pressure factor

Materials correction factor

Module factor

(includes all of the supporting equipment and connections and installation)

Purchased cost of heat exchanger in 1987

(mild steel construction, shell and tube floating head)

CE index for process equipment in 1987

CE index for process equipment in 1995

21,903

146.96

1.165

Installed cost of heat exchanger in 1995

$\$ 180,000$

$\$ 784,068$

6. Heat Exchanger 5

$\mathrm{Q}=\operatorname{Load}(\mathrm{Btu} / \mathrm{h})$

Tha $=$ Inlet temperature of hot fluid $\left({ }^{\circ} \mathrm{F}\right)$

$\mathrm{Thb}=$ Outlet temperature of hot fluid $\left({ }^{\circ} \mathrm{F}\right)$

Pressure of hot gases (psia)

$\mathrm{Tca}=$ Inlet temperature of cold fluid $\left({ }^{\circ} \mathrm{F}\right)$

$\mathrm{Tcb}=$ Outlet temperature of cold fluid $\left({ }^{\circ} \mathrm{F}\right)$

Delta T1

Delta T2

Log mean temperature difference $\left({ }^{\circ} \mathrm{F}\right)$

Overall heat transfer coefficient (Btu/h/ft ${ }^{\circ} /{ }^{\circ} \mathrm{F}$ )

Heat transfer area $\left(\mathrm{ft}^{2}\right)$

Operating pressure (psia)

Pressure factor

Materials correction factor

Module factor

(includes all of the supporting equipment and connections and installation)

Purchased cost of heat exchanger in 1987

(mild steel construction, shell and tube floating head)

CE index for process equipment in 1987

CE index for process equipment in 1995

$310,639,429$

450.00

150

150

121.4

356.77

93.23133627 
7. Cathode Gas Expansion Turbine

Turbine size (hp)

Purchased cost in 1987

(assumes that the cost of expansion turbine is same as that of a compressor of similar size)

Module factor

CE index for process equipment in 1987

CE index for process equipment in 1995

Installed cost of turbine in 1995

104,190

$\$ 10,432,285$

1.00

$\$ 320$

$\$ 374$

$\$ 12,189,473$

14.70

$\$ 150$

224.43

$\$ 24,374,545$

1.00

320

1995373.9

$\$ 28,480,133$

\section{Steam Turbine}

Turbine output (MW)

165.77

The cost of steam turbine is already included in base case.

10. Condenser

$Q=\operatorname{Load}(B t u / h)$

$\mathrm{Tha}=$ Inlet temperature of hot fluid $\left({ }^{\circ} \mathrm{F}\right)$

$398,353,905$

121.36

121

Pressure of hot gases (psia)

Tca $=$ Inlet temperature of cold fluid $\left({ }^{\circ} \mathrm{F}\right)$

2

70.0

$\mathrm{Tcb}=$ Outlet temperature of cold fluid $\left({ }^{\circ} \mathrm{F}\right)$

100.00

Delta $T 1$

Delta T2

21.35924367

Log mean temperature difference $\left({ }^{\circ} \mathrm{F}\right)$

Overall heat transfer coefficient $\left(\mathrm{Btu} / \mathrm{h} / \mathrm{ft} \mathrm{t}^{2}{ }^{\circ} \mathrm{F}\right)$

Heat transfer area $\left(\mathrm{ft}^{2}\right)$

Operating pressure (psia)

23,300

146.96

1.165

Materials correction factor

Module factor

(includes all of the supporting equipment and connections and installation)

Purchased cost of heat exchanger in 1987

(mild steel construction, shell and tube floating head)

$\$ 190,000$

CE index for process equipment in 1987

CE index for process equipment in 1995

373.9 
TABLE 7.6 (Cont.)

11. Pump

Horsepower

106

Size exponent

Purchased cost in 1987

(includes motor, coupling, base:cast iron, horizontal)

Module factor

CE index for process equipment in 1987

CE index for process equipment in 1995

$\$ 21,035$

Installed cost of pump in 1995

$\$ 12,000$

1.5

320

373.9

12. Fuel Cell Stack

Fuel cell power output (kW)

Unit cost per kilowatt

77,989

$\$ 180$

Total cost

$\$ 14,038,020$

13. Fuel Cell Invertor

Unit cost per kilowatt

$\$ 100$

Total cost

$\$ 7,798,900$

14. Fuel Cell Controls

Unit cost per kilowatt

$\$ 140$

Total cost

$\$ 10,918,460$

15. Fuel Cell and Component Assembly

Unit cost per kilowatt

$\$ 110$

Total cost

$\$ 8,578,790$

Total Direct Cost

$\$ 93,973,387$

Total Direct Cost for Three Trains 
TABLE 7.7 Sizing and Cost Estimation for Major Equipment Used for $\mathrm{CO}_{2}$ Removal in Membrane Process in Case 4

1. First-Stage Membranes

Membrane area $\left(\mathrm{ft}^{2}\right)$

$2,346,506$

Unit cost of membrane

$\$ 13.00$

Total cost

$\$ 30,504,579$

2. Second-Stage Membranes

Membrane area $\left(\mathrm{ft}^{2}\right)$

Unit cost of membrane

$1,287,497$

Total cost

$\$ 13.00$

3. Compressor between First and Second Stages

Inlet pressure (psia)

Outlet pressure (psia)

25.00

Compressor size (hp)

150.00

9,747

Purchased cost of reciprocating compressor in 1987

(includes electric motor drive and gear reducer)

Size factor for compressor

$\$ 1,600,000$

Materials correction factor

Module factor

CE index for process equipment in 1987

CE index for process equipment in 1995

Installed cost of compressor in 1995

$\$ 16,737,465$

Recycle Compressor

Inlet pressure (psia)

140.00

Outlet pressure (psia)

150.00

Compressor size (hp)

46

Purchased cost of reciprocating compressor in 1987

$\$ 38,000$

(includes electric motor drive and gear reducer)

Size factor for compressor

Materials correction factor

Module factor

CE index for process equipment in 1987

CE index for process equipment in 1995 
5. Heat Exchanger after Compressor

$Q=\operatorname{Load}(B t u / h)$

Tha $=$ Inlet temperature of hot fluid $\left({ }^{\circ} \mathrm{F}\right)$

$19,116,496$

472.66

$\mathrm{Thb}=$ Outlet temperature of hot fluid $\left({ }^{\circ} \mathrm{F}\right)$

212

Pressure of hot gases (psia)

150

$\mathrm{Tca}=$ Inlet temperature of cold fluid $\left({ }^{\circ} \mathrm{F}\right)$

70.00

$\mathrm{Tcb}=$ Outlet temperature of cold fluid $\left({ }^{\circ} \mathrm{F}\right)$

150.00

Delta T1

322.66

Delta $T 2$

142

220

Log mean temperature difference $\left({ }^{\circ} \mathrm{F}\right)$

30

Overall heat transfer coefficient $\left(\mathrm{Btu} / \mathrm{h} / \mathrm{ft}^{2} /{ }^{\circ} \mathrm{F}\right)$

2,895

Heat transfer area $\left(\mathrm{ft}^{2}\right)$

Operating pressure (psia)

445

1.08

Pressure factor

Materials correction factor

Module factor

(includes all of the supporting equipment and connections and installation)

Purchased cost of heat exchanger in 1987

(mild steel construction; shell and tube floating head)

CE index for process equipment in 1987

$C E$ index for process equipment in 1995

Installed cost of heat exchanger in 1995

$\$ 201,906$

6. $\mathrm{CO}_{2}$ Product Gas Compressors

Compressor 1 (hp)

Compressor $2(\mathrm{hp})$

Compressor 3 (hp)

Purchased cost of centrifugal compressor 1 in 1987

Purchased cost of centrifugal compressor 2 in 1987

Purchased cost of centrifugal compressor 3 in 1987

(includes electric motor drive and gear reducer)

Size factor for compressor

Module factor

4,276

4,276

4,276

$\$ 900,000$

$\$ 900,000$

$\$ 900,000$

CE index for process equipment in 1987

CE index for process equipment in 1995

$\$ 2,734,144$

$\$ 2,734,144$

Installed cost of Compressor 2 in 1995

$\$ 2,734,144$

Total Direct Cost

$\$ 162,204,286$

Total Direct Cost for Three Trains

$\$ 486,612,859$ 


\section{$8 \mathrm{CO}_{2}$ Pipeline Transport and Sequestering}

\subsection{Pipeline Transport of $\mathrm{CO}_{2}$}

Once the $\mathrm{CO}_{2}$ has been recovered from the fuel-gas stream, its transportation, utilization, and disposal remain significant issues. In a previous study for METC (Doctor et al. 1994), the issues associated with the transport and sequestering of $\mathrm{CO}_{2}$ were considered in greater detail; that information serves as the basis for this work. The $\mathrm{CO}_{2}$ represents a large-volume, relatively lowvalue by-product that cannot be sequestered in the same way as most coal-utilization wastes (i.e., by landfilling). Large volumes of recovered $\mathrm{CO}_{2}$ are likely to be moved by pipeline, and if sequestering were required, new pipelines would likely need to be constructed. In some cases, existing pipelines could be used, perhaps in a shared mode with other products. Costs for pipeline construction and use vary greatly on a regional basis within the United States. The recovered $\mathrm{CO}_{2}$ represents more than 3 million normal cubic meters per day of gas volume. It is assumed that the transport and sequestering process releases approximately $2 \%$ of the recovered $\mathrm{CO}_{2}$.

\section{$8.2 \mathrm{CO}_{2}$ Sequestering}

Proposals have been made to dispose of $\mathrm{CO}_{2}$ in the ocean depths. However, many questions of engineering and ecological concern associated with such options remain unanswered, and the earliest likely reservoir is a land-based geological repository (Hangebrauck 1992). A portion of the $\mathrm{CO}_{2}$ can be used for enhanced oil recovery, which sequesters a portion of the $\mathrm{CO}_{2}$, or the $\mathrm{CO}_{2}$ can be completely sequestered in depleted gas/oil reservoirs and nonpotable aquifers. Both the availability of these zones and the technical and economic limits to their use need to be better characterized. Levelized costs were prepared; they take into account that the power required for compression will rise throughout the life cycle of these sequestering reservoirs. The first reservoirs to be used will, in fact, be capable of accepting all IGCC $\mathrm{CO}_{2}$ gas for a 30-year period without requiring any additional compression costs for operation. The pipeline transport and sequestering process represents approximately 26 mills/kWh for the $\mathrm{CO}_{2}$-recovery cases. 


\section{Conclusions - Energy Cycle/Economic Comparisons}

\subsection{Energy Consumption and $\mathrm{CO}_{2}$ Emissions}

An adjustment of $9.7 \%$ between the oxygen-blown and air-blown KRW IGCC cases was needed to make the coal feed rates match. A second minor adjustment was required because the design basis coal was different for these two sets of studies. Efficiencies calculated previously were matched, while the $\mathrm{CO}_{2}$ emission rates for the air-blown cases decreased slightly by $4.2 \%$.

Data on energy consumption and $\mathrm{CO}_{2}$ emissions for all seven cases appear in Tables 9.19.7. The IGCC power plant performance and emission factors within traditional battery limits have been bounded to clarify what in the net energy cycle falls outside the plant battery. The most significant contributor to the net $\mathrm{CO}_{2}$ emissions for the $\mathrm{CO}_{2}$-recovery cases is the makeup power to match the base case performance.

\subsection{Capital Costs for KRW Integrated Gasification Combined-Cycle Power Generation}

Capital costs for each of the IGCC power plants appear in Tables 9.8-9.13. For convenience in comparison, the $\mathrm{O}_{2}$-blown and air-blown cases are next to each other. The large cost difference between these two systems for the coal preparation system is a consequence of the fact that the airblown system employs the sulfator section off-gases for coal drying. The $\mathrm{O}_{2}$-blown case requires an air-separation system and compression. Here the air-blown case is lower in cost as a consequence of needing only compression. From this section of the plant forward, the $\mathrm{O}_{2}$-blown case shows lower costs for comparable plant subsystems as a consequence of the reduced gas volumes being handled.

Whenever a standard turn-key package system was part of the design, a zero percent contingency was taken. In addition, throughout the study, the Handy-Whitman Index was employed to bring all capital estimates to a fourth quarter of 1994 dollar basis. The plant cost for the $\mathrm{O}_{2}$-blown base case comes to $\$ 1,332 / \mathrm{kW}$; for the air-blown case, it is slightly lower, at $\$ 1,253 / \mathrm{kW}$. For the optimal $\mathrm{O}_{2}$-blown $\mathrm{CO}_{2}$-recovery case, this cost increases to $\$ 1,687 / \mathrm{kW}$, while for the optimal air-blown $\mathrm{CO}_{2}$-recovery case, this cost rises to $\$ 1,773 / \mathrm{kW}$.

\subsection{Costs of Electricity}

The costs of electricity appear in Tables 9.14-9.20. Following this, Table 9.21 summarizes the major costs for each of the combined-cycle cases. For the air-blown cases, the cost of limestone and the cost of ash disposal have been adjusted to typical values as given by the TAG study (EPRI 1993). A comparison of the cost of electricity for the $\mathrm{CO}_{2}$-release base cases found the cost of the air-blown IGCC case to be 58.29 mills $/ \mathrm{kWh}$ and the cost of the $\mathrm{O}_{2}$-blown IGCC case to be 56.86 mills $/ \mathrm{kWh}$. There was no clear advantage for the optimal cases employing glycol $\mathrm{CO}_{2}$ recovery; the cost of the air-blown IGCC was $95.48 \mathrm{mills} / \mathrm{kWh}$, and the cost of the $\mathrm{O}_{2}$-blown case was slightly lower, at $94.55 \mathrm{mills} / \mathrm{kWh}$. 
TABLE 9.1 Energy Consumption and $\mathrm{CO}_{2}$ Emissions for Oxygen-Blown Base Case: KRW IGCC with $\mathrm{No}^{\mathrm{CO}_{2}}$ Recovery

Mining and Transport

Raw Coal in Mine

Caal Rail Transport

Subtotal

IGCC Power Plant

Coal Preparation

Gasifier Island

Power Island

Subtotal

Power - Gas Turbine

Power - Steam Turbine

GROSS Power

NET Power

Pipeline/Sequester

Energy Cycle Power Use

NET Energy Cycle

$\mathrm{CO} 2$ emission rate/net cycle

Power use/ $\mathrm{CO} 2$ in reservoir
Electricity $\mathrm{CO} 2$ release

MW $\mathrm{kg} / \mathrm{h}$

$-2.36 \quad 2,356$

$-0.05 \quad 523$

$-2.41 \quad 2,879$

$-0.85$

$-36.82$

$-7.02$

6,153

$-44.70$

320,387

326,540

298.80

159.40

458.20

413.50

0.00

0

$-47.11$

411.09

329,419

$0.801 \mathrm{~kg} \mathrm{CO2} / \mathrm{kWh}$ $\mathrm{N} / \mathrm{A} \quad \mathrm{kWh} / \mathrm{kg} \mathrm{CO} 2$ 
TABLE 9.2 Energy Consumption and $\mathrm{CO}_{2}$ Emissions for Air-Blown Base Case: KRW IGCC with No

$\mathrm{CO}_{2}$ Recovery

Mining and Transport

Raw Coal in Mine

Coal Rail Transport

Limestone Mining

Limestone Rail Tansport

Subtotal

IGCC Power Plant

Coal/Limestone Preparation

Gasifier Island

Power Island

Subtotal

Power - Gas Turbine

Power - Steam Turbine

GROSS Power

NET Power

Pipeline/Sequester

Energy Cycle Power Use

NET Energy Cycle

$\mathrm{CO} 2$ emission rate/net cycle

Power use/ $\mathrm{CO} 2$ in reservoir.

\begin{tabular}{|c|c|}
\hline $\begin{array}{l}\text { Electricity } \\
\text { MW }\end{array}$ & $\begin{array}{l}\mathrm{CO} 2 \text { release } \\
\mathrm{kg} / \mathrm{h}\end{array}$ \\
\hline-2.36 & 2,356 \\
\hline-0.05 & 523 \\
\hline-0.25 & 250 \\
\hline-0.02 & 156 \\
\hline-2.67 & 3,286 \\
\hline
\end{tabular}

$-3.49$

$-20.12$

$-10.58$

$-34.19$

11,374

137

315,029

326,540

302.66

176.97

479.63

445.44

0.00

0

$-36.87$

$442.76 \quad 329,825$

$0.745 \mathrm{~kg} \mathrm{CO} 2 / \mathrm{kWh}$

$\mathrm{N} / \mathrm{A} \quad \mathrm{kWh} / \mathrm{kg} \mathrm{CO} 2$ 
TABLE 9.3 Energy Consumption and $\mathrm{CO}_{2}$ Emissions for Case 1: Oxygen-Blown KRW IGCC with Glycol $\mathrm{CO}_{2}$ and $\mathrm{H}_{2} \mathrm{~S}$ Recovery and Gas Turbine Topping Cycle

Mining and Transport

Electricity $\mathrm{CO} 2$ release

Raw Coal in Mine MW $\mathrm{kg} / \mathrm{h}$

Coal Rail Transport

$-2.36 \quad 2,356$

Subtotal

$-0.05 \quad 523$

$-2.41 \quad 2,879$

\section{IGCC Power Plant}

Coal Preparation

$-0.85$

Gasifier Island

$-36.82$

Power Island

$-7.02$

320,387

Glycol Circulation

$-5.80 \quad-260,055$

Glycol Refrigeration

$-4.50$

Power Recovery Turbines

3.40

$\mathrm{CO} 2$ Compression (to 2100psi)

$-17.30$

Subtotal

$-68.90$

66,485

Power - Gas Turbine

284.80

Power - Steam Turbine

161.60

GROSS Power

446.40

NET Power

377.50

Pipeline/Sequester

Pipeline $\mathrm{CO} 2$

Pipeline booster stations

Geological reservoir (2\% loss)

Subtotal

$-1.64$

260,055

$0.00 \quad-254,854$

$-1.64 \quad 6,839$

Energy Cycle Power Use

$-72.95$

NET Energy Cycle

373.45

Derating from O2-Base Case

37.64

Make-up Power

37.64

76,202

TOTAL

411.09

37,637

113,840

$\mathrm{CO} 2$ emission rate/net cycle Power use/ $\mathrm{CO} 2$ in reservoir

$0.277 \mathrm{~kg} \mathrm{CO} 2 / \mathrm{kWh}$

$0.148 \mathrm{kWh} / \mathrm{kg} \mathrm{CO} 2$ 
TABLE 9.4 Energy Consumption and $\mathrm{CO}_{2}$ Emissions for Case 2: Oxygen-Blown KRW IGCC with Membrane $\mathrm{CO}_{2}$ Recovery, Glycol $\mathrm{H}_{2} \mathrm{~S}$ Recovery, and Gas Turbine Topping Cycle

Mining and Transport

Raw Coal in Mine

Coal Rail Transport

Subtotal

IGCC Power Plant

Coal Preparation

Gasifier Island

Power Island

Glycol Circulation

Glycol Refrigeration

Membrane Compression

$\mathrm{CO} 2$ Compression (10 2100psi)

Subtotal

Power - Gas Turbine

Power - Steam Turbine

GROSS Power

NET Power

Pipeline/Sequester

Pipeline $\mathrm{CO} 2$

Pipeline booster stations

Geological reservoir (2\% loss)

Subtotal

Energy Cycle Power Use

NET Energy Cycle

Derating from O2-Base Case

Make-up Power

TOTAL

$\mathrm{CO} 2$ emission rate/net cycle

Power use/ $\mathrm{CO} 2$ in reservoir
Electricity $\mathrm{CO} 2$ release

MW

$\mathrm{kg} / \mathrm{h}$

$\begin{array}{lr}-2.36 & 2,356 \\ -0.05 & 523 \\ -2.41 & 2,879\end{array}$

$-2.41 \quad 2,879$

$-0.85$

$-36.82$

6,153

$-7.02$

320,387

$-0.90$

$-232,505$

$-3.00$

$-19.00$

$-20.00$

$-87.60$

94,034

262.80

154.80

417.60

330.00 
TABLE 9.5 Energy Consumption and $\mathrm{CO}_{2}$ Emissions for Case 3: Oxygen-Blown KRW IGCC with Glycol $\mathrm{CO}_{2}$ Recovery, Methanol $\mathrm{H}_{2} \mathrm{~S}$ Recovery, and Fuel Cell Topping Cycle

Mining and Transport

Raw Coal in Mine

Coal Rail Transport

Subtotal
Electricity $\mathrm{CO} 2$ release

MW $\mathrm{kg} / \mathrm{h}$ $-2.36 \quad 2,356$ $-0.05 \quad 523$

$-2.41 \quad 2,879$

\begin{tabular}{|c|c|c|}
\hline \multicolumn{3}{|l|}{ IGCC Power Plant } \\
\hline Coal Preparation & -0.85 & \\
\hline Gasifier Island & -36.82 & 6,153 \\
\hline Power Island & -11.24 & 320,387 \\
\hline CO2 Recovery & -4.54 & $-260,055$ \\
\hline $\mathrm{CO} 2$ Compression (to $2100 \mathrm{psi}$ ) & -24.93 & \\
\hline Subtotal & -78.39 & 66,485 \\
\hline Power - Gas Turbine & 246.70 & \\
\hline Power - Steam Turbine & 171.80 & \\
\hline GROSS Power & 418.50 & \\
\hline NET Power & 340.11 & \\
\hline \multicolumn{3}{|l|}{ Pipeline/Sequester } \\
\hline Pipeline $\mathrm{CO} 2$ & & 260,055 \\
\hline Pipeline booster stations & -1.64 & 1,637 \\
\hline Geological reservoir (2\% loss) & 0.00 & $-254,854$ \\
\hline Subtotal & -1.64 & 6,839 \\
\hline Energy Cycle Power Use & -82.44 & \\
\hline NET Energy Cycle & 336.06 & 76,202 \\
\hline Derating from O2-blown Base Case & 75.03 & \\
\hline Make-up Power & 75.03 & 75,030 \\
\hline TOTAL & 411.09 & 151,232 \\
\hline CO2 emission rate/net cycle & \multirow{2}{*}{\multicolumn{2}{|c|}{$\begin{array}{l}0.368 \mathrm{~kg} \mathrm{CO} 2 / \mathrm{kWh} \\
75.03 \mathrm{MW}\end{array}$}} \\
\hline CO2 Sequestering power use & & \\
\hline Power use/CO2 in reservoir & \multicolumn{2}{|c|}{$0.294 \mathrm{kWh} / \mathrm{kg} \mathrm{CO} 2$} \\
\hline
\end{tabular}


TABLE 9.6 Energy Consumption and $\mathrm{CO}_{2}$ Emissions for Case 4: Oxygen-Blown KRW IGCC with Membrane $\mathrm{CO}_{2}$ Recovery, Methanol $\mathrm{H}_{2} \mathrm{~S}$ Recovery, and Fuel Cell Topping Cycle

Electricity $\mathrm{CO} 2$ release

Mining and Transport

Raw Coal in Mine

MW

$\mathrm{kg} / \mathrm{h}$

Coal Rail Transport

$-2.36 \quad 2,356$

$-0.05 \quad 523$

Subtotal

$-2.41 \quad 2,879$

IGCC Power Plant

Coal Preparation

$-0.85$

$-36.82$

6,153

Gasifier Island

$-11.22$

320,387

CO2 Recovery

$-21.80$

$-272,137$

$\mathrm{CO} 2$ Compression (to 2100psi)

$-28.70$

Subtotal

$-99.40$

54,403

Power - Fuel Cells

247.40

Power - Steam Turbine

165.80

GROSS Power

413.20

NET Power

313.80

Pipeline/Sequester

Pipeline $\mathrm{CO} 2$

Pipeline booster stations

$-1.71$

272,137

Geological reservoir (2\% loss)

0.00

1,713

Subtotal

$-1.71$

$-266,694$

Energy Cycle Power Use

7,156

NET Energy Cycle

$-103.52$

Derating from O2-blown Base Case

309.68

101.41

Make-up Power

101.41

411.09

64,438

TOTAL

$0.403 \mathrm{~kg} \mathrm{CO} 2 / \mathrm{kWh}$

$\mathrm{CO} 2$ emission rate/net cycle

$\mathrm{CO} 2$ Sequestering power use

Power use/ $\mathrm{CO} 2$ in reservoir

$$
\begin{aligned}
& 101.41 \mathrm{MW} \\
& 0.380 \mathrm{kWh} / \mathrm{kg} \mathrm{CO} 2
\end{aligned}
$$


TABLE 9.7 Energy Consumption and $\mathrm{CO}_{2}$ Emissions for Optimal Air-Blown Case: KRW IGCC with Glycol $\mathrm{CO}_{2}$ Recovery, In-Bed $\mathrm{H}_{2} \mathrm{~S}$ Recovery, and Gas Turbine

Topping Cycle

Mining and Transport

Raw Coal in Mine

Coal Rail Transport

Limestone Mining

Limestone Rail Tansport

Subtotal

IGCC Power Plant

Coal/Limestone Preparation

Gasifier Island

Power Island

$\mathrm{CO} 2$ Recovery

$\mathrm{CO} 2$ Compression (to 2100psi)

Subtotal

Power - Gas Turbine

Power - Steam Turbine

GROSS Power

NET Power

Pipeline/Sequester

Pipeline $\mathrm{CO} 2$

Pipeline booster stations

Geological reservoir (2\% loss)

Subtotal

Energy Cycle Power Use

NET Energy Cycle

Derating from O2-blown Base Case

Make-up Power

TOTAL

$\mathrm{CO} 2$ emission rate/net cycle

$\mathrm{CO} 2$ Sequestering power use

Power use/CO2 in reservoir
Electricity $\mathrm{CO} 2$ release

MW $\mathrm{kg} / \mathrm{h}$

$-2.36 \quad 2,356$

$-0.05 \quad 523$

$-0.25 \quad 250$

$-0.02 \quad 156$

$-2.67 \quad 3,286$

$-3.49 \quad 11,374$
-21.11

$-21.11$

137

$-11.10 \quad 315,029$

$-17.21 \quad-285,499$

$-32.21$

$-85.11$

41,041

274.39

186.50

460.88

375.77 
TABLE 9.8 Capital Costs for Air-Blown and Oxygen-Blown Base Cases with No $\mathrm{CO}_{2}$ Recovery

\begin{tabular}{|c|c|c|c|c|}
\hline & & $\begin{array}{c}\text { KRW O2-Blown } \\
\text { Base Case } \\
413.50 \mathrm{MW} \\
\end{array}$ & & $\begin{array}{c}\text { KRW Air-Blown } \\
\text { Base Case } \\
445.44 \mathrm{MW} \\
\end{array}$ \\
\hline System & cont.* & Capital Cost, \$K & cont.* & Capital Cost, \$K \\
\hline Direct Costs & & & & \\
\hline Coal Handling \& Preparation & $0.0 \%$ & $\$ 8,339$ & $0.0 \%$ & $\$ 18,208$ \\
\hline Limestone Handling \& Prep. & & & $0.0 \%$ & $\$ 10,388$ \\
\hline Air-Separation Plant/Comprs. & $0.0 \%$ & $\$ 66,249$ & $0.0 \%$ & $\$ 10,099$ \\
\hline Gasification & $20.0 \%$ & $\$ 99,714$ & $20.0 \%$ & $\$ 118,866$ \\
\hline Fines and Ash Handling & $15.0 \%$ & $\$ 2,650$ & $15.0 \%$ & $\$ 6,628$ \\
\hline Acid Gas Treatment (H2S) & $10.0 \%$ & $\$ 12,286$ & $10.0 \%$ & $\$ 37,902$ \\
\hline Sulfur Recovery (Claus) & $0.0 \%$ & $\$ 6,777$ & & \\
\hline Tail-Gas Treatment (SCOT) & $0.0 \%$ & $\$ 6,116$ & & \\
\hline Sour-water Stripping & $10.0 \%$ & $\$ 4,408$ & & \\
\hline Wastewater Treatment & $30.0 \%$ & $\$ 5,116$ & & \\
\hline Gas Turbine System & $5.0 \%$ & $\$ 77,837$ & $5.0 \%$ & $\$ 80,654$ \\
\hline HRSG System & $5.0 \%$ & $\$ 25,808$ & $5.0 \%$ & $\$ 28,407$ \\
\hline Steam Turbine System & $0.0 \%$ & $\$ 47,900$ & $0.0 \%$ & $\$ 52,722$ \\
\hline Indirect Costs $\quad$ Sub-total & & $\$ 363,199$ & & $\$ 363,873$ \\
\hline General Facilities & $10.5 \%$ & $\$ 38,136$ & $10.5 \%$ & $\$ 38,207$ \\
\hline Engineering Fees & $8.0 \%$ & $\$ 29,056$ & $8.0 \%$ & $\$ 29,110$ \\
\hline Process Contingency & $7.9 \%$ & $\$ 28,727$ & $9.3 \%$ & $\$ 34,011$ \\
\hline Project Contingency & $20.0 \%$ & $\$ 91,823$ & $20.0 \%$ & $\$ 93,040$ \\
\hline Sub-total & & $\$ 187,742$ & & $\$ 194,367$ \\
\hline Total Plant Cost-TPC & & $\$ 550,941$ & & $\$ 558,241$ \\
\hline $\operatorname{Cost(\$ /kW-net~output)}$ & & $\$ 1,332$ & & $\$ 1,253$ \\
\hline Interest \& Inflation (AFUDC) ${ }^{* *}$ & $20.5 \%$ & $\$ 112,943$ & $20.5 \%$ & $\$ 114,439$ \\
\hline Total Plant Investment-TPI & & $\$ 663,884$ & & $\$ 672,680$ \\
\hline Royalties & $0.6 \%$ & $\$ 2,179$ & $0.6 \%$ & \$2,183 \\
\hline Initial Inventory & $3.3 \%$ & $\$ 11,986$ & $3.3 \%$ & $\$ 12,008$ \\
\hline Start-up Costs & $4.6 \%$ & $\$ 16,707$ & $4.6 \%$ & $\$ 16,738$ \\
\hline Spare Parts & $2.2 \%$ & $\$ 7,990$ & $2.2 \%$ & $\$ 8,005$ \\
\hline $\begin{array}{l}\text { Working Capital } \\
\text { TOTAL }\end{array}$ & $3.3 \%$ & $\begin{array}{r}\$ 11,986 \\
\$ 714,731\end{array}$ & $3.3 \%$ & $\begin{array}{r}\$ 12,008 \\
\$ 723,622\end{array}$ \\
\hline
\end{tabular}


TABLE 9.9 Capital Costs for Case 1: Oxygen-Blown KRW IGCC with Glycol $\mathrm{CO}_{2}$ and $\mathrm{H}_{2} \mathrm{~S}$ Recovery and Gas

Turbine Topping Cycle

\begin{tabular}{|c|c|c|}
\hline & & $\begin{array}{c}\text { Net Power } \\
\text { Case \#1 } \\
377.5 \mathrm{MW}\end{array}$ \\
\hline System & cont.* & Capital Cost, $\$ K$ \\
\hline \multicolumn{3}{|l|}{ Direct Costs } \\
\hline Coal Handling \& Preparation & $0.0 \%$ & $\$ 8,339$ \\
\hline Air-Separation Plant/Comprs. & $0.0 \%$ & $\$ 66,249$ \\
\hline Gasification & $20.0 \%$ & $\$ 99,714$ \\
\hline Fines and Ash Handling & $15.0 \%$ & $\$ 2,650$ \\
\hline Glycol (H2S) & $10.0 \%$ & $\$ 17,756$ \\
\hline Sulfur Recovery (Claus) & $0.0 \%$ & $\$ 6,777$ \\
\hline Tail-Gas Treatment (SCOT) & $0.0 \%$ & $\$ 6,116$ \\
\hline Sour-water Stripping & $10.0 \%$ & $\$ 4,408$ \\
\hline Shift System & $10.0 \%$ & $\$ 21,571$ \\
\hline Glycol (CO2 Recovery) & $10.0 \%$ & \$28,597 \\
\hline Wastewater Treatment & $30.0 \%$ & $\$ 5,116$ \\
\hline Gas Turbine System & $5.0 \%$ & $\$ 77,837$ \\
\hline HRSG System & $5.0 \%$ & $\$ 25,808$ \\
\hline Steam Turbine System & $0.0 \%$ & $\$ 47,900$ \\
\hline \multicolumn{2}{|l|}{ Sub-total } & $\$ 418,838$ \\
\hline \multicolumn{3}{|l|}{ Indirect Costs } \\
\hline General Facilities & $10.5 \%$ & $\$ 43,978$ \\
\hline Engineering Fees & $8.0 \%$ & $\$ 33,507$ \\
\hline Process Contingency & $8.2 \%$ & $\$ 34,291$ \\
\hline Project Contingency & $20.0 \%$ & $\$ 106,123$ \\
\hline \multicolumn{2}{|l|}{ Sub-total } & $\$ 217,898$ \\
\hline Total Plant Cost-TPC & & $\$ 636,737$ \\
\hline Cost $(\$ / k W$-net output $)$ & & $\$ 1,687$ \\
\hline Interest \& Inflation (AFUDC) ${ }^{* *}$ & $20.5 \%$ & $\$ 130,531$ \\
\hline Total Plant Investment-TPI & & $\$ 767,268$ \\
\hline Royalties & $0.6 \%$ & $\$ 2,513$ \\
\hline Initial Inventory & $3.3 \%$ & $\$ 13,822$ \\
\hline Start-up Costs & $4.6 \%$ & $\$ 19,267$ \\
\hline Spare Parts & $2.2 \%$ & $\$ 9,214$ \\
\hline Working Capital & $3.3 \%$ & $\$ 13,822$ \\
\hline TOTAL & & $\$ 825,905$ \\
\hline
\end{tabular}


TABLE 9.10 Capital Costs for Case 2: Oxygen-Blown $\mathrm{KRW}$ IGCC with Membrane $\mathrm{CO}_{2}$ Recovery, Glycol $\mathrm{H}_{2} \mathrm{~S}$ Recovery, and Gas Turbine Topping Cycle

\begin{tabular}{|c|c|c|}
\hline & & $\begin{array}{c}\text { Net Power } \\
\text { Case \#2 } \\
330.0 \mathrm{MW}\end{array}$ \\
\hline System & cont* & Capital Cost, \$K \\
\hline Direct Costs & & \\
\hline Coal Handling \& Preparation & $0.0 \%$ & $\$ 8,339$ \\
\hline Air-Separation Plant/Comprs. & $0.0 \%$ & $\$ 66,249$ \\
\hline Gasification & $20.0 \%$ & $\$ 99,714$ \\
\hline Fines and Ash Handling & $15.0 \%$ & $\$ 2,650$ \\
\hline Glycol (H2S) & $10.0 \%$ & $\$ 17,756$ \\
\hline Sulfur Recovery (Claus) & $0.0 \%$ & $\$ 6,777$ \\
\hline Tail-Gas Treatment (SCOT) & $0.0 \%$ & $\$ 6,116$ \\
\hline Sour-water Stripping & $10.0 \%$ & $\$ 4,408$ \\
\hline Shift System & $10.0 \%$ & $\$ 19,980$ \\
\hline Membrane (CO2 Recovery) & $10.0 \%$ & $\$ 110,448$ \\
\hline Wastewater Treatment & $30.0 \%$ & $\$ 5,116$ \\
\hline Gas Turbine System & $5.0 \%$ & $\$ 77,837$ \\
\hline HRSG System & $5.0 \%$ & $\$ 25,808$ \\
\hline Steam Turbine System & $0.0 \%$ & $\$ 47,900$ \\
\hline Sub-total & & $\$ 499,097$ \\
\hline Indirect Costs & & \\
\hline General Facilities & $10.5 \%$ & $\$ 52,405$ \\
\hline Engineering Fees & $8.0 \%$ & $\$ 39,928$ \\
\hline Process Contingency & $8.5 \%$ & $\$ 42,316$ \\
\hline Project Contingency & $20.0 \%$ & $\$ 126,749$ \\
\hline Sub-total & & $\$ 261,399$ \\
\hline Total Plant Cost-TPC & & $\$ 760,496$ \\
\hline $\operatorname{Cost}(\$ / k W-n e t$ output $)$ & & $\$ 2,305$ \\
\hline Interest \& Inflation (AFUDC) ${ }^{* *}$ & $20.5 \%$ & $\$ 155,902$ \\
\hline Total Plant Investment-TPI & & $\$ 916,397$ \\
\hline Royalties . & $0.6 \%$ & $\$ 2,995$ \\
\hline Initial Inventory & $3.3 \%$ & $\$ 16,470$ \\
\hline Start-up Costs & $4.6 \%$ & $\$ 22,958$ \\
\hline Spare Parts & $2.2 \%$ & $\$ 10,980$ \\
\hline Working Capital & $3.3 \%$ & $\$ 16,470$ \\
\hline TOTAL & & $\$ 986,271$ \\
\hline
\end{tabular}


TABLE 9.11 Capital Costs for Case 3: Oxygen-Blown KRW IGCC with Glycol $\mathrm{CO}_{2}$ Recovery, Methanol $\mathrm{H}_{2} \mathrm{~S}$ Recovery, and Fuel Cell Topping Cycle

\begin{tabular}{|c|c|c|}
\hline & & $\begin{array}{l}\text { Net Power } \\
\text { Case \#3 } \\
340.11 \mathrm{MW}\end{array}$ \\
\hline System & cont.* & Capital Cost, \$K \\
\hline \multicolumn{3}{|l|}{ Direct Costs } \\
\hline Coal Handling \& Preparation & $0.0 \%$ & $\$ 8,339$ \\
\hline Air-Separation Plant/Comprs. & $0.0 \%$ & $\$ 66,249$ \\
\hline Gasification & $20.0 \%$ & $\$ 99,714$ \\
\hline Fines and Ash Handling & $15.0 \%$ & $\$ 2,650$ \\
\hline Chilled Methanol (H2S) & $10.0 \%$ & $\$ 22,825$ \\
\hline Sulfur Recovery (Claus) & $0.0 \%$ & $\$ 6,777$ \\
\hline Tail-Gas Treatment (SCOT) & $0.0 \%$ & $\$ 6,116$ \\
\hline Sour-water Stripping & $10.0 \%$ & $\$ 4,408$ \\
\hline Glycol (CO2 Recovery) & $10.0 \%$ & $\$ 31,555$ \\
\hline Wastewater Treatment & $30.0 \%$ & $\$ 5,116$ \\
\hline Molten Carbonate Fuel Cells & $15.0 \%$ & $\$ 285,637$ \\
\hline Steam Turbine System & $0.0 \%$ & $\$ 47,900$ \\
\hline Sub-total & & $\$ 587,286$ \\
\hline \multicolumn{3}{|l|}{ Indirect Costs } \\
\hline General Facilities & $10.5 \%$ & $\$ 61,665$ \\
\hline Engineering Fees & $8.0 \%$ & $\$ 46,983$ \\
\hline Process Contingency & $12.0 \%$ & $\$ 70,599$ \\
\hline Project Contingency & $20.0 \%$ & $\$ 153,307$ \\
\hline Sub-total & & $\$ 332,554$ \\
\hline Total Plant Cost-TPC & & $\$ 919,840$ \\
\hline $\operatorname{Cost}(\$ / k W$-net output $)$ & & $\$ 2,705$ \\
\hline Interest \& Inflation (AFUDC) ${ }^{* *}$ & $20.5 \%$ & $\$ 188,567$ \\
\hline Total Plant Investment-TPI & & $\$ 1,108,407$ \\
\hline Royalties & $0.6 \%$ & $\$ 3,524$ \\
\hline Initial Inventory & $3.3 \%$ & $\$ 19,380$ \\
\hline Start-up Costs & $4.6 \%$ & $\$ 27,015$ \\
\hline Spare Parts & $2.2 \%$ & $\$ 12,920$ \\
\hline Working Capital & $3.3 \%$ & $\$ 19,380$ \\
\hline TOTAL & & $\$ 1,190,627$ \\
\hline
\end{tabular}


TABLE 9.12 Capital Costs for Case 4: Oxygen-Blown KRW IGCC with Membrane $\mathrm{CO}_{2}$ Recovery, Methanol $\mathrm{H}_{2} \mathrm{~S}$ Recovery, and Fuel Cell Topping Cycle

\begin{tabular}{|c|c|c|}
\hline & & $\begin{array}{l}\text { Net Power } \\
\text { Case \#4 } \\
313.77 \mathrm{MW}\end{array}$ \\
\hline System & cont.* & Capital Cost, \$K \\
\hline Direct Costs & & \\
\hline Coal Handling \& Preparation & $0.0 \%$ & $\$ 8,339$ \\
\hline Air-Separation Plant/Comprs. & $0.0 \%$ & $\$ 66,249$ \\
\hline Gasification & $20.0 \%$ & $\$ 99,714$ \\
\hline Fines and Ash Handling & $15.0 \%$ & $\$ 2,650$ \\
\hline Chilled Methanol (H2S) & $10.0 \%$ & $\$ 22,825$ \\
\hline Sulfur Recovery (Claus) & $0.0 \%$ & $\$ 6,777$ \\
\hline Tail-Gas Treatment (SCOT) & $0.0 \%$ & $\$ 6,116$ \\
\hline Sour-water Stripping & $10.0 \%$ & $\$ 4,408$ \\
\hline CO2 Recovery - Membrane & $10.0 \%$ & $\$ 181,868$ \\
\hline Wastewater Treatment & $30.0 \%$ & $\$ 5,116$ \\
\hline Molten Carbonate Fuel Cells & $15.0 \%$ & $\$ 281,920$ \\
\hline Steam Turbine System & $0.0 \%$ & $\$ 47,900$ \\
\hline Sub-total & & $\$ 733,882$ \\
\hline General Facilities & $10.5 \%$ & $\$ 77,058$ \\
\hline Engineering Fees & $8.0 \%$ & $\$ 58,711$ \\
\hline Process Contingency & $11.6 \%$ & $\$ 85,073$ \\
\hline Project Contingency & $20.0 \%$ & $\$ 190,945$ \\
\hline Sub-total & & $\$ 411,786$ \\
\hline Total Plant Cost-TPC & & $\$ 1,145,668$ \\
\hline $\operatorname{Cost}(\$ / k W-n e t$ output $)$ & & $\$ 3,651$ \\
\hline Interest \& Inflation (AFUDC) ${ }^{* *}$ & $20.5 \%$ & $\$ 234,862$ \\
\hline Total Plant Investment-TPI & & $\$ 1,380,529$ \\
\hline Royalties & $0.6 \%$ & $\$ 4,403$ \\
\hline Initial Inventory & $3.3 \%$ & $\$ 24,218$ \\
\hline Start-up Costs & $4.6 \%$ & $\$ 33,759$ \\
\hline Spare Parts & $2.2 \%$ & $\$ 16,145$ \\
\hline Working Capital & $3.3 \%$ & $\$ 24,218$ \\
\hline TOTAL & & $\$ 1,483,273$ \\
\hline
\end{tabular}


TABLE 9.13 Capital Costs for Optimal Air-Blown Case: $\mathrm{KRW}$ IGCC with Glycol $\mathrm{CO}_{2}$ Recovery, In-Bed $\mathrm{H}_{2} \mathrm{~S}$

Recovery, and Gas Turbine Topping Cycle

\begin{tabular}{|c|c|c|}
\hline & & $\begin{array}{l}\text { Net Power } \\
\text { Glycol CO2 } \\
375.77 \text { MW }\end{array}$ \\
\hline System & cont.* & Capital Cost, \$K \\
\hline Direct Costs & & \\
\hline Coal Handling \& Preparation & $0.0 \%$ & $\$ 18,208$ \\
\hline Limestone Handling \& Prep. & $0.0 \%$ & $\$ 10,388$ \\
\hline Air-Separation Plant/Comprs. & $0.0 \%$ & $\$ 10,099$ \\
\hline Gasification & $20.0 \%$ & $\$ 118,866$ \\
\hline Fines and Ash Handling & $15.0 \%$ & $\$ 6,628$ \\
\hline Glycol H2S & $10.0 \%$ & $\$ 37,902$ \\
\hline Shift/Glycol CO2/Compression & $10.0 \%$ & $\$ 60,321$ \\
\hline Gas Turbine System & $5.0 \%$ & $\$ 80,654$ \\
\hline HRSG System & $5.0 \%$ & $\$ 28,407$ \\
\hline Steam Turbine System & $0.0 \%$ & $\$ 52,722$ \\
\hline Sub-total & & $\$ 424,194$ \\
\hline Indirect Costs & & \\
\hline General Facilities & $10.5 \%$ & $\$ 44,540$ \\
\hline Engineering Fees & $8.0 \%$ & $\$ 33,936$ \\
\hline Process Contingency & $9.4 \%$ & $\$ 40,043$ \\
\hline Project Contingency & $20.0 \%$ & $\$ 108,542$ \\
\hline Sub-total & & $\$ 227,061$ \\
\hline Total Plant Cost-TPC & & $\$ 651,255$ \\
\hline Cost $(\$ / k W$-net output $)$ & & $\$ 1,733$ \\
\hline Interest \& Inflation (AFUDC) ${ }^{* *}$ & $20.5 \%$ & $\$ 133,507$ \\
\hline Total Plant Investment-TPI & & $\$ 784,762$ \\
\hline Royalties & $0.6 \%$ & $\$ 2,545$ \\
\hline Initial Inventory & $3.3 \%$ & $\$ 13,998$ \\
\hline Start-up Costs & $4.6 \%$ & $\$ 19,513$ \\
\hline Spare Parts & $2.2 \%$ & $\$ 9,332$ \\
\hline Working Capital & $3.3 \%$ & $\$ 13,998$ \\
\hline TOTAL & & $\$ 844,149$ \\
\hline
\end{tabular}


TABLE 9.14 Operating Costs for Oxygen-Blown Base Case: KRW IGCC with No $\mathrm{CO}_{2}$ Recovery

$$
\text { Net Power }(M W)=
$$

Capacity factor=

Annual Net Power Production (MW) =

Net Energy-cycle Power $(\mathrm{MW})=$

\begin{tabular}{|c|c|c|c|c|}
\hline OPERATING COSTS & Basis & Unit Cost & & Annual Cost \\
\hline Fuel - Illinois \#6 Coal (ROM) & $4,110 \mathrm{~T} / \mathrm{D}$ & $\$ 35.00$ & $\$ / \mathrm{T}$ & $\$ 34,126,136$ \\
\hline Coal - prepared & 3,845 T/D & & & \\
\hline \multicolumn{5}{|l|}{ Consumable material } \\
\hline Catalyst, etc. & & & & $\$ 1,640,000$ \\
\hline Miscellaneous & & & & $\$ 603,730$ \\
\hline Ash/Sorbent Disposal & $491.4 \mathrm{~T} / \mathrm{D}$ & $\$ 11.00$ & $S / T$ & $\$ 1,282,432$ \\
\hline \multicolumn{5}{|l|}{ Plant Labor } \\
\hline Oper Labor (w benefits) & $23.0 \mathrm{men} / \mathrm{shift}$ & $\$ 25.50$ & $\$ / \mathrm{h}$ & $\$ 5,137,198$ \\
\hline Supervision/support & $25 \%$ of above & & & $\$ 1,284,300$ \\
\hline Maintenance & $2.7 \%$ of Direct & & & $\$ 9,806,370$ \\
\hline Insurance \& Local Taxes & $0.9 \%$ of Direct & & & $\$ 3,268,790$ \\
\hline Other - \% of Oper Labor & $12.5 \%$ of above & & & $\$ 642,150$ \\
\hline By-Product Credit & $102.1 \mathrm{TPD}$ & $\$ 30.00$ & $\mathrm{~S} / \mathrm{T}$ & $(\$ 726,857)$ \\
\hline Net Operating Cost & & & & $\$ 22,938,113$ \\
\hline \multicolumn{5}{|l|}{ COSTS OF ELECTRICITY } \\
\hline Levelizing Factors & Constant (\$) & Basis $(\mathbf{K \$})$ & & Annual $(K \$)$ \\
\hline Capital Charge & 0.111 & $\$ 714,731$ & & $\$ 137,253$ \\
\hline Fuel & 1.025 & $\$ 34,126$ & & \\
\hline Operating \& Maintenance & 1.000 & $\$ 22,938$ & & \\
\hline Cost of Electricity - Levelized & mills/kWh & & & \\
\hline Capital Charge & 33.70 & & & \\
\hline Fuel & 14.86 & & & \\
\hline Operating \& Maintenance & 9.74 & & & \\
\hline Total Cost of Electricity & 58.29 & Basis (MW) & 413.5 & \\
\hline Energy-cycle Cost of Electricity & 58.64 & Basis (MW) & 411.1 & \\
\hline
\end{tabular}


TABLE 9.15 Operating Costs for Air-Blown Base Case: KRW IGCC with No $\mathrm{CO}_{2}$ Recovery

Net Power $(M W)=$ 445.44

Capacity factor=

Annual Net Power Production (MW)= Net Energy-cycle Power (MW)=

\begin{tabular}{|c|c|c|c|c|}
\hline OPERATING COSTS & Basis & Unit Cost & & Annual Cost \\
\hline Fuel - Illinois \#6 Coal (ROM) & $4109.7 \mathrm{~T} / \mathrm{D}$ & $\$ 35.00$ & $\$ / T$ & $\$ 34,126,164$ \\
\hline Coal - prepared & $3,845 \mathrm{~T} / \mathrm{D}$ & & & \\
\hline \multicolumn{5}{|l|}{ Consumable material } \\
\hline Limestone & $1100.8 \mathrm{~T} / \mathrm{D}$ & $\$ 11.20$ & $\$ / T$ & $\$ 2,925,032$ \\
\hline Nahcolite & $4.9 \mathrm{~T} / \mathrm{D}$ & $\$ 261.25$ & $\$ / T$ & $\$ 301,676$ \\
\hline Zinc Ferrite & $1.1 \mathrm{~T} / \mathrm{D}$ & $\$ 6,270.00$ & $\$ / T$ & $\$ 1,659,216$ \\
\hline Miscellaneous & & & & $\$ 603,730$ \\
\hline Ash/Sorbent Disposal & $1248.2 \mathrm{~T} / \mathrm{D}$ & $\$ 11.00$ & $S / T$ & $\$ 3,257,569$ \\
\hline \multicolumn{5}{|l|}{ Plant Labor } \\
\hline Oper Labor ( $w$ benefits) & $23.0 \mathrm{men} / \mathrm{shift}$ & $\$ 25.50$ & $S / h$ & $\$ 5,137,198$ \\
\hline Supervision/support & $25 \%$ of above & & & $\$ 1,284,300$ \\
\hline Maintenance & $2.7 \%$ of Direct & & & $\$ 9,824,580$ \\
\hline Insurance \& Local Taxes & $0.9 \%$ of Direct & & & $\$ 3,274,860$ \\
\hline Other - \% of Oper Labor & $12.5 \%$ of above & & & $\$ 642,150$ \\
\hline By-Product Credit & & & & SO \\
\hline Net Operating Cost & & & & $\$ 28,910,311$ \\
\hline \multicolumn{5}{|l|}{ COSTS OF ELECTRICITY } \\
\hline Levelizing Factors & Constant (\$) & Basis $(\mathrm{K} \$)$ & & Annual (K\$) \\
\hline Capital Charge & 0.111 & $\$ 723,622$ & & $\$ 144,212$ \\
\hline Fuel & 1.025 & $\$ 34,126$ & & \\
\hline Operating \& Maintenance & 1.000 & $\$ 28,910$ & & \\
\hline Cost of Electricity - Levelized & mills/kWh & & & \\
\hline Capital Charge & 31.67 & & & \\
\hline Fuel & 13.79 & & & \\
\hline Operating \& Maintenance & 11.40 & & & \\
\hline Total Cost of Electricity & 56.86 & Basis (MW) & 445.4 & \\
\hline Energy-cycle Cost of Electricity & 57.40 & Basis (MW) & 441.3 & \\
\hline
\end{tabular}


TABLE 9.16 Operating Costs for Case 1: Oxygen-Blown KRW IGCC with Glycol $\mathrm{CO}_{2}$ and $\mathrm{H}_{2} \mathrm{~S}$ Recovery and Gas Turbine Topping Cycle

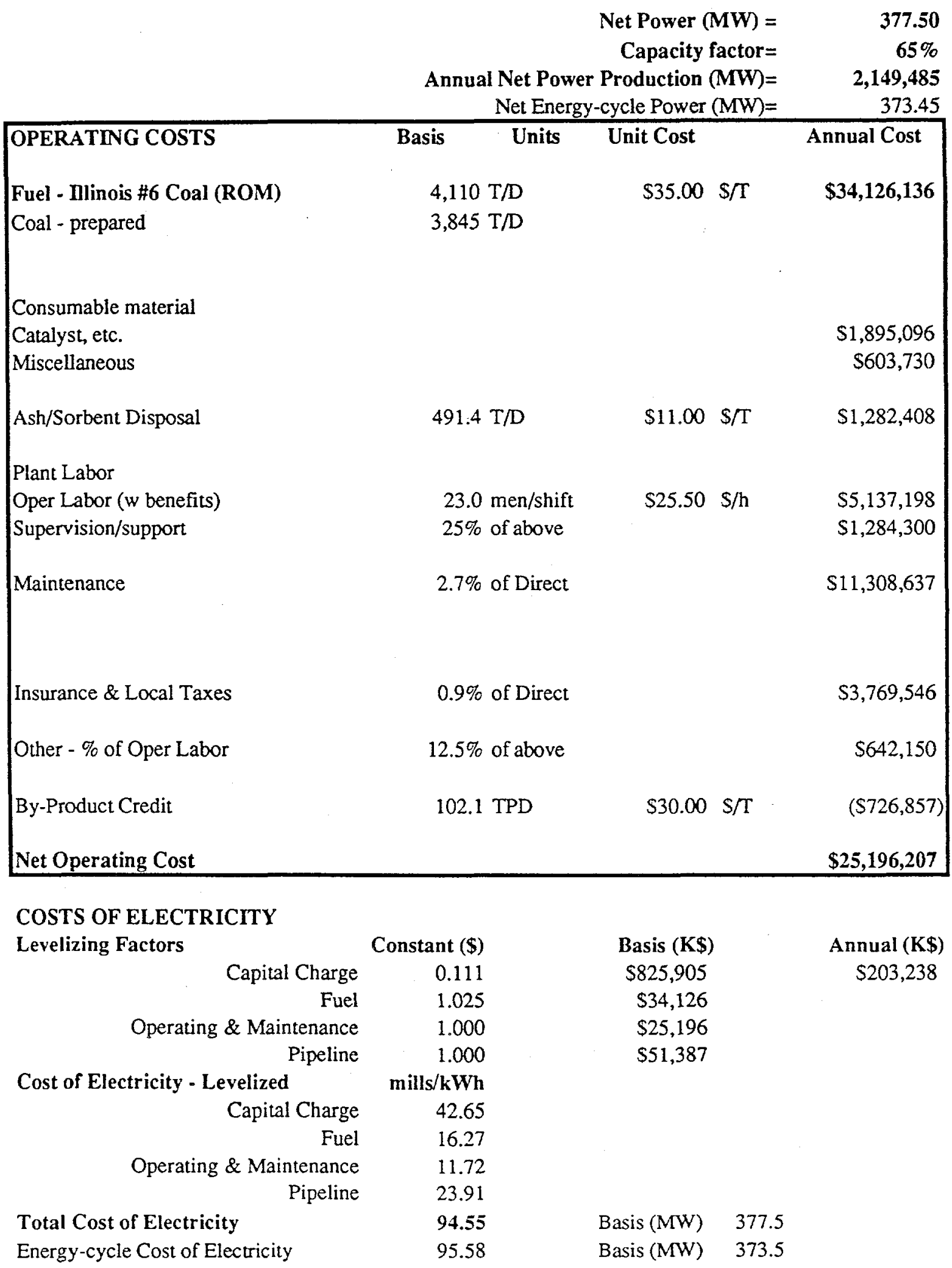


TABLE 9.17 Operating Costs for Case 2: Oxygen-Blown KRW IGCC with Membrane $\mathrm{CO}_{2}$ Recovery, Glycol $\mathrm{H}_{2} \mathrm{~S}$ Recovery, and Gas Turbine Topping Cycle

Net Power $(\mathbf{M W})=$

330.00

Capacity factor $=$

$65 \%$

Annual Net Power Production (MW)=

Net Energy-cycle Power (MW) =

\begin{tabular}{|c|c|c|c|c|}
\hline OPERATING COSTS & Basis & Unit Cost & & Annual Cost \\
\hline Fuel - Illinois \#6 Coal (ROM) & $4,110 \mathrm{~T} / \mathrm{D}$ & $\$ 35.00$ & $\$ / T$ & $\$ 34,126,136$ \\
\hline Coal - prepared & $3,845 \mathrm{~T} / \mathrm{D}$ & & & \\
\hline \multicolumn{5}{|l|}{ Consumable material } \\
\hline Catalyst, etc. & & & & $\$ 1,895,096$ \\
\hline Miscellaneous & & & & $\$ 603,730$ \\
\hline Ash/Sorbent Disposal & $491.4 \mathrm{~T} / \mathrm{D}$ & $\$ 11.00$ & $\mathrm{~S} / \mathrm{T}$ & $\$ 1,282,408$ \\
\hline \multicolumn{5}{|l|}{ Plant Labor } \\
\hline Oper Labor ( $w$ benefits) & $23.0 \mathrm{men} / \mathrm{shift}$ & $\$ 25.50$ & 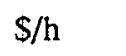 & $\$ 5,137,198$ \\
\hline Supervision/support & $25 \%$ of above & & & $\$ 1,284,300$ \\
\hline Maintenance & $2.7 \%$ of Direct & & & $\$ 13,475,622$ \\
\hline Membrane Replacement (6 yr) & $16.7 \%$ of capital & & & $\$ 18,407,981$ \\
\hline Insurance \& Local Taxes & $0.9 \%$ of Direct & & & $\$ 4,491,874$ \\
\hline Other - \% of Oper Labor & $12.5 \%$ of above & & & $\$ 642,150$ \\
\hline By-Product Credit & 102.1 TPD & $\$ 30.00$ & $\$ / T$ & $(\$ 726,857)$ \\
\hline Net Operating Cost & & & & $\$ 46,493,502$ \\
\hline \multicolumn{5}{|l|}{ COSTS OF ELECTRICITY } \\
\hline Levelizing Factors & Constant $(\$)$ & Basis $(\mathbf{K} \$)$ & & Annual (K\$) \\
\hline Capital Charge & 0.111 & $\$ 986,271$ & & $\$ 242,336$ \\
\hline Fuel & 1.025 & $\$ 34,126$ & & \\
\hline Operating \& Maintenance & 1.000 & $\$ 46,494$ & & \\
\hline $\begin{array}{lll}\text { Pipeline } & \end{array}$ & 1.000 & $\$ 51,387$ & & \\
\hline Cost of Electricity - Levelized & mills/kWh & & & \\
\hline Capital Charge & 58.26 & & & \\
\hline Fuel & 18.62 & & & \\
\hline Operating \& Maintenance & 24.74 & & & \\
\hline Pipeline & 27.35 & & & \\
\hline Total Cost of Electricity & 128.97 & Basis (MW) & 330.0 & \\
\hline Energy-cycle Cost of Electricity & 144.26 & Basis (MW) & 295.0 & \\
\hline
\end{tabular}


TABLE 9.18 Operating Costs for Case 3: Oxygen-Blown KRW IGCC with Glycol $\mathrm{CO}_{2}$ Recovery, Methanol $\mathrm{H}_{2} \mathrm{~S}$ Recovery, and Fuel Cell Topping Cycle

Net Power $(\mathrm{MW})=$ 340.11

Capacity factor= $65 \%$ Annual Net Power Production (MW)= $1,936,586$ Net Energy-cycle Power (MW)=

\begin{tabular}{|c|c|c|c|c|}
\hline OPERATING COSTS & Basis & Unit Cost & & Annual Cost \\
\hline Fuel - Illinois \#6 Coal (ROM) & $4,110 \mathrm{~T} / \mathrm{D}$ & $\$ 35.00$ & $\$ / T$ & $\$ 34,126,136$ \\
\hline Coal - prepared & $3,845 \mathrm{~T} / \mathrm{D}$ & & & \\
\hline \multicolumn{5}{|l|}{ Consumable material } \\
\hline Catalyst, etc. & & & & $\$ 1,895,096$ \\
\hline Miscellaneous & & & & $\$ 603,730$ \\
\hline Ash/Sorbent Disposal & $491.4 \mathrm{~T} / \mathrm{D}$ & $\$ 11.00$ & $\$ / T$ & $\$ 1,282,408$ \\
\hline \multicolumn{5}{|l|}{ Plant Labor } \\
\hline Oper Labor ( $w$ benefits) & $23.0 \mathrm{men} / \mathrm{shift}$ & $\$ 25.50$ & $\$ / \mathrm{h}$ & $\$ 5,137,198$ \\
\hline Supervision/support & $25 \%$ of above & & & $\$ 1,284,300$ \\
\hline Maintenance & $2.7 \%$ of Direct & & & $\$ 15,856,718$ \\
\hline Insurance \& Local Taxes & $0.9 \%$ of Direct & & & S5,285,573 \\
\hline Other - \% of Oper Labor & $12.5 \%$ of above & & & $\$ 642,150$ \\
\hline By-Product Credit & $102.1 \mathrm{TPD}$ & $\$ 30.00$ & $\$ / T$ & $(\$ 726,857)$ \\
\hline Net Operating Cost & & & & $\$ 31,260,315$ \\
\hline \multicolumn{5}{|l|}{ COSTS OF ELECTRICITY } \\
\hline Levelizing Factors & Constant (\$) & Basis $(\mathbf{K} \$)$ & & Annual (K\$) \\
\hline Capital Charge & 0.111 & $\$ 1,190,627$ & & $\$ 249,786$ \\
\hline Fuel & 1.025 & $\$ 34,126$ & & \\
\hline Operating \& Maintenance & 1.000 & $\$ 31,260$ & & \\
\hline Pipeline & 1.000 & $\$ 51,387$ & & \\
\hline Cost of Electricity - Levelized & mills/kWh & & & \\
\hline Capital Charge & 68.24 & & & \\
\hline Fuel & 18.06 & & & \\
\hline Operating \& Maintenance & 16.14 & & & \\
\hline Pipeline & 26.53 & & & \\
\hline Total Cost of Electricity & 128.98 & Basis (MW) & 340.1 & \\
\hline Energy-cycle Cost of Electricity & 130.54 & Basis (MW) & 336.1 & \\
\hline
\end{tabular}


TABLE 9.19 Operating Costs for Case 4: Oxygen-Blown KRW IGCC with Membrane $\mathrm{CO}_{2}$ Recovery, Methanol $\mathrm{H}_{2} \mathrm{~S}$ Recovery, and Fuel Cell Topping Cycle

\begin{tabular}{|c|c|c|c|c|}
\hline & \multicolumn{3}{|c|}{$\begin{array}{r}\text { Net Power }(\mathrm{MW})= \\
\text { Capacity factor }=\end{array}$} & $\begin{array}{r}313.77 \\
65 \%\end{array}$ \\
\hline & \multicolumn{3}{|c|}{ Annual Net Power Production $(M W)=$} & $1,786,606$ \\
\hline & \multicolumn{3}{|c|}{ Net Energy-cycle Power $(\mathrm{MW})=$} & 309.41 \\
\hline OPERATING COSTS & Units & Unit Cost & & Annual Cost \\
\hline Fuel - Illinois \#6 Coal (ROM) & $4,110 \mathrm{~T} / \mathrm{D}$ & $\$ 35.00$ & $\$ / T$ & $\$ 34,126,136$ \\
\hline Coal - prepared & $3,845 \mathrm{~T} / \mathrm{D}$ & & & \\
\hline \multicolumn{5}{|l|}{ Consumable material } \\
\hline Catalyst, etc. & & & & $\$ 1,895,096$ \\
\hline Miscellaneous & & & & $\$ 603,730$ \\
\hline Ash/Sorbent Disposal & $491.4 \mathrm{~T} / \mathrm{D}$ & $\$ 11.00$ & $S / T$ & $\$ 1,282,408$ \\
\hline \multicolumn{5}{|l|}{ Plant Labor } \\
\hline Oper Labor ( $w$ benefits) & $23.0 \mathrm{men} / \mathrm{shift}$ & $\$ 25.50$ & $\$ / h$ & $\$ 5,137,198$ \\
\hline Supervision/support & $25 \%$ of above & & & $\$ 1,284,300$ \\
\hline Maintenance & $2.7 \%$ of Direct & & & $\$ 19,814,807$ \\
\hline Insurance \& Local Taxes & $0.9 \%$ of Direct & & & $\$ 6,604,936$ \\
\hline Other - \% of Oper Labor & $12.5 \%$ of above & & & $\$ 642,150$ \\
\hline By-Product Credit & $102.1 \mathrm{TPD}$ & $\$ 30.00$ & $\$ / \mathrm{T}$ & $(\$ 726,857)$ \\
\hline Net Operating Cost & & & & $\$ 36,537,767$ \\
\hline \multicolumn{5}{|l|}{ COSTS OF ELECTRICITY } \\
\hline Levelizing Factors & Constant $(\$)$ & Basis $(\mathbf{K \$})$ & & Annual (K\$) \\
\hline Capital Charge & 0.111 & $\$ 1,483,273$ & & $\$ 287,547$ \\
\hline Fuel & 1.025 & $\$ 34,126$ & & \\
\hline Operating \& Maintenance & 1.000 & $\$ 36,538$ & & \\
\hline Pipeline & 1.000 & $\$ 51,387$ & & \\
\hline Cost of Electricity - Levelized & mills/kWh & & & \\
\hline Capital Charge & 92.15 & & & \\
\hline Fuel & 19.58 & & & \\
\hline Operating \& Maintenance & 20.45 & & & \\
\hline Pipeline & 28.76 & & & \\
\hline Total Cost of Electricity & 160.95 & Basis (MW) & 313.8 & \\
\hline Energy-cycle Cost of Electricity & 163.21 & Basis (MW) & 309.4 & \\
\hline
\end{tabular}


TABLE 9.20 Operating Costs for Optimal Air-Blown Case: KRW IGCC with Glycol $\mathrm{CO}_{2}$ Recovery, In-Bed $\mathrm{H}_{2} \mathrm{~S}$ Recovery, and Gas Turbine Topping Cycle

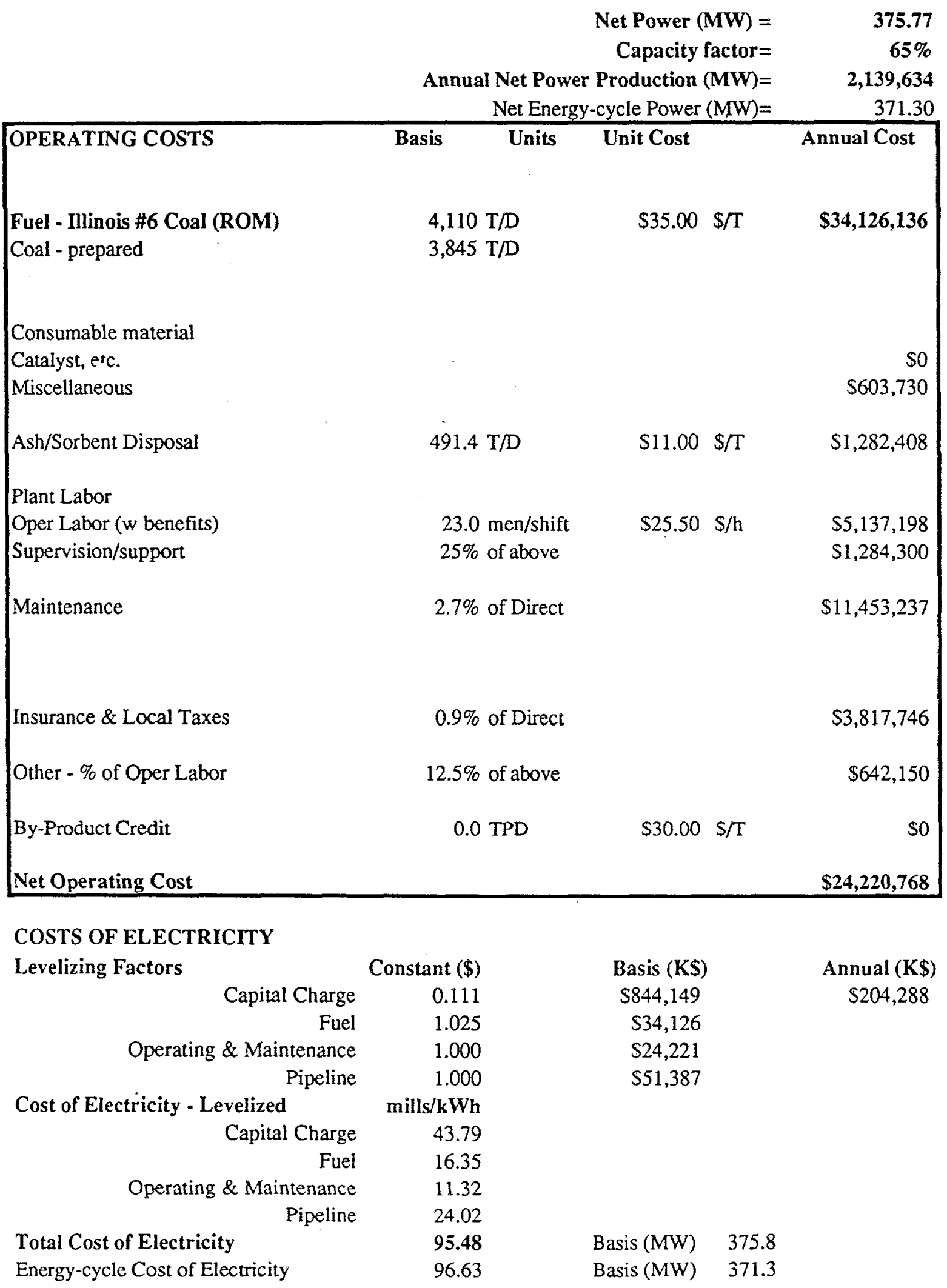


TABLE 9.21 Summary of Comparative Costs of IGCC Systems

\begin{tabular}{|c|c|c|c|c|c|c|c|c|}
\hline Case & & BASE & BASE & Case \#1 & Case \#2 & Case\#3 & Case \#4 & ESD-24/Glycol \\
\hline Gasifier Oxidant & & Oxygen & Air & Oxygen & Oxygen & Oxygen & Oxygen & Air \\
\hline H2S Recovery & & Glycol & In-Bed/ZnTi & Glycol & Glyco! & Methanol & Methanol & $\mathrm{In}-\mathrm{Bcd} / \mathrm{ZnTi}$ \\
\hline CO2 Recovery & & none & none & Glycol & Membrane & Glycol & Membrane & Glycol \\
\hline Topping Cycle & & Turbine & Turbine & Turbine & Turbine & Fuel Cell & Fuel Cell & Turbine \\
\hline Bottoming Cycle & & Steam & Steam & Steam & Steam & Steam & Steam & Steam \\
\hline Component & Unit & & & & & & & \\
\hline Base Plant Capital & $\$ / \mathrm{kW}$ & $\$ 1,332$ & $\$ 1,253$ & $\$ 1,485$ & $\$ 1,703$ & $\$ 2,560$ & $\$ 2,746$ & $\$ 1,487$ \\
\hline CO2 Control Capital & $\$ / \mathrm{kW}$ & $\$()$ & $\$ 0$ & $\$ 202$ & $\$ 602$ & $\$ 145$ & $\$ 905$ & $\$ 246$ \\
\hline Total Plant Capital & $\$ / k W$ & $\$ 1,332$ & $\$ 1,253$ & $\$ 1,687$ & $\$ 2,305$ & $\$ 2,705$ & $\$ 3,651$ & $\$ 1,733$ \\
\hline Power Plant Annual Cost & $\$ \mathrm{~K}$ & $\$ 137,253$ & $\$ 144,212$ & $\$ 203,238$ & $\$ 242,336$ & $\$ 249,786$ & $\$ 287,547$ & $\$ 204,288$ \\
\hline Power Cost & & & & & & & & \\
\hline Base Plant Power Cost & mills/kWh & 58.29 & 56.86 & 70.64 & 101.62 & 102.45 & 132.19 & 71.46 \\
\hline Pipeline Cost & mills $/ \mathrm{kWh}$ & 0 & 0 & 23.91 & 27.35 & 26.53 & 28.76 & 24.02 \\
\hline Net Power Cost & mills/kWh & 58.29 & 56.86 & 94.55 & 128.97 & 128.98 & 160.95 & 95.48 \\
\hline Coal Encrgy Input & $10^{\wedge} 6 \mathrm{Btu} / \mathrm{h}$ & 3839 & 3839 & 3839 & 3839 & 3839 & 3839 & 3839 \\
\hline Gross Power Output & MW & 458.20 & 479.63 & 446.40 & 417.60 & 418.50 & 413.20 & 460.88 \\
\hline In Plant Power Use & MW & 44.70 & 34.19 & 68.90 & 87.60 & 78.39 & 99.40 & 85.11 \\
\hline Net Plant Output & MW & 413.50 & 445.44 & 377.50 & 330.00 & 340.11 & 313.80 & 375.77 \\
\hline Net Heat Rate & $\mathrm{Btu} / \mathrm{kWh}$ & 9284 & 8618 & 10170 & 11633 & 11288 & 12234 & 10216 \\
\hline Thermal Efliciency - HHV & $\%$ & $36.78 \%$ & $39.62 \%$ & $33.58 \%$ & $29.35 \%$ & $30.25 \%$ & $27.91 \%$ & $33.42 \%$ \\
\hline Out of Plant Power Use & MW & 2.41 & 4.18 & 4.05 & 3.87 & 4.05 & 4.12 & 4.47 \\
\hline Net Energy Cycle Power & MW & 411.09 & 441.26 & 373.45 & 326.13 & 336.06 & 309.68 & 371.30 \\
\hline Net Energy Cycle Heat Rate & Btu/kWh & 9339 & 8700 & 10280 & 11771 & 11424 & 12397 & 10339 \\
\hline Thermal Efficiency - HHV & $\%$ & $36.56 \%$ & $39.25 \%$ & $33.21 \%$ & $29.01 \%$ & $29.89 \%$ & $27.54 \%$ & $33.02 \%$ \\
\hline Net Encrgy Cycle Power & MW & 411.09 & 441.26 & 373.45 & 326.13 & 336.06 & 309.68 & 371.30 \\
\hline Net Replacemenl [Added] Power & MW & 0.00 & $(30.17)$ & 37.64 & 84.96 & 75.03 & 101.41 & 39.79 \\
\hline Net Grid Power & MW & 411.09 & 411.09 & 411.09 & 411.09 & 411.09 & 411.09 & 411.09 \\
\hline
\end{tabular}




\section{References}

Clinton, W.J., and A. Gore, Jr., 1993, The Climate Change Action Plan, Washington, D.C., Oct.

Doctor, R.D., et al., 1994, Gasification Combined Cycle: Carbon Dioxide Recovery, Transport, and Disposal, ANL/ESD-24, Argonne National Laboratory, Argonne, Ill., Sept.

EPRI, 1993, Technical Assessment Guide (TAG) Volume 1: Electricity Supply-1993, Electric Power Research Institute, Palo Alto, Calif.

Gallaspy, D.T., et al., 1990a, Southern Company Service's Study of a KRW-Based GCC Power Plant, EPRI GS-6876, Electric Power Research Institute, Palo Alto, Calif., July.

Gallaspy, D.T., et al., 1990b, Assessment of Coal Gasification/Hot Gas Cleanup Based Advanced Gas Turbine Systems: Final Report, DOE/MC/26019.3004 (DE91002084), prepared by Southern Company Services, Inc., Birmingham, Ala., et al., for U.S. Department of Energy, Morgantown Energy Technology Center, Morgantown, W.Va., Dec.

Hangebrauck, R.P., et al., 1992, "Carbon Dioxide Sequestration," in Proceedings of the 1992 Greenhouse Gas Emissions and Mitigation Research Symposium, sponsored by the U.S. Environmental Protection Agency, Washington, D.C., Aug. 18-20.

Hendricks, C., 1994, Carbon Dioxide Removal from Coal-Fired Power Plants, Kluwer Academic Publishers, Dordrecht, the Netherlands.

Smith, A., 1994, "Norway Pioneers Large Scale $\mathrm{CO}_{2}$ Disposal in 1996," Greenhouse Issues, International Energy Agency, Glouchestershire, U.K., Aug.

Stone, R., 1994, "Most Nations Miss the Mark on Emission-Control Plans," Science 226(5193): 1939, Dec. 23. 
DISTRIBUTION FOR ANL/ESD-34

Internal

ANL Publications and Record Services Dept.

R. Weeks

L. Welko (5)

R.D. Doctor (102)

M. Moniger

\section{External}

U.S. Department of Energy Office of Scientific and Technical Information (12) Manager, U.S. Department of Energy Chicago Field Office ANL-E Libraries

ANL-W Library 
